\title{
Synthesis and characterization of molecularly imprinted polymers and their application in preconcentrators for gas phase sensors
}

Yi Fu

West Virginia University

Follow this and additional works at: https://researchrepository.wvu.edu/etd

\section{Recommended Citation}

$\mathrm{Fu}, \mathrm{Yi}$, "Synthesis and characterization of molecularly imprinted polymers and their application in preconcentrators for gas phase sensors" (2003). Graduate Theses, Dissertations, and Problem Reports. 1921.

https://researchrepository.wvu.edu/etd/1921

This Dissertation is protected by copyright and/or related rights. It has been brought to you by the The Research Repository @ WVU with permission from the rights-holder(s). You are free to use this Dissertation in any way that is permitted by the copyright and related rights legislation that applies to your use. For other uses you must obtain permission from the rights-holder(s) directly, unless additional rights are indicated by a Creative Commons license in the record and/ or on the work itself. This Dissertation has been accepted for inclusion in WVU Graduate Theses, Dissertations, and Problem Reports collection by an authorized administrator of The Research Repository @ WVU.

For more information, please contact researchrepository@mail.wvu.edu. 
Synthesis and Characterization of Molecularly Imprinted Polymers and Their Application in Preconcentrators for Gas Phase Sensors

\author{
Yi Fu \\ Dissertation submitted to the \\ College of Arts and Sciences \\ at West Virginia University \\ in partial fulfillment of the requirements for the degree of \\ Doctor of Philosophy \\ in
}

Chemistry

Harry O. Finklea, Ph.D., Chair,

Fred L. King, Ph.D.

Joseph Shaeiwitz, Ph.D.

Kenneth Showalter, Ph.D.

Aaron Timperman, Ph.D.

Department of Chemistry

Morgantown, West Virginia

2003

Keywords: Molecularly Imprinted Polymer, Quartz Crystal Microbalance, Organic Vapor, Gas Phase Sensor, Partition Coefficient, Adsorption Capacity, Selectivity, Breakthrough, Preconcentrator, Thermal Desorption

Copyright 2003 Yi Fu 


\section{ABSCTRACT \\ Synthesis and Characterization of Molecularly Imprinted Polymers and Their Application in Preconcentrators for Gas Phase Sensors}

\section{Yi Fu}

Various of molecularly imprinted polymers were synthesized by different protocols. Piezoelectric quartz crystals coated with molecularly imprinted polymers were prepared to detect small organic vapors. Hydroquinone (HQ) and phenol (P) have been used as non-covalent bound templates in order to generate shapeselectivity cavities in the polymer matrix. The recognition film was immobilized on the crystal surface via a pre-coated poly(isobutylene) layer. The selective behaviors of the imprinted polymer films were studied by their steady-state response to various kinds of small organic vapors. The partition coefficients of polymers toward vapors were evaluated. The imprinted polymers exhibit high sensitivity and selectivity toward organic vapors as toluene and benzene. Imprinted polymers prepared by different synthesis schemes were compared. The influence of template concentration and the polymer components was also investigated. The adsorption capacity of molecularly imprinted polymers was characterized and compared by breakthrough studies. From our results, molecularly imprinted polymer is promising for the development of selective piezoelectric sensor for organic vapor detection.

Different types of preconcentrator devices capable of pre-concentrating organic vapors at low ppm levels were fabricated and described. The target organic vapors were enriched onto a small bed of adsorbents and subsequently released by thermal desorption scheme. Solid adsorbents (Tenax GR, TA and molecularly imprinted polymers) were evaluated for possible use in a preconcentrator.

Approximate preconcentration factor in the range of several thousand can be achieved by using the block polymer imprinted with hydroquinone. 


\section{Acknowledgements}

I would like to express my gratitude to my advisor, Dr. Harry O. Finklea, for his support and guidance throughout my years at West Virginia University. I would also like to thank my other committee members, Dr. King, Dr. Showalter, Dr. Shaeiwitz and Dr. Timperman for reviewing my research proposal and my dissertation. Much appreciation is also expressed to the past and current Finklea group members (Rob, Chris, Mark and Zhaoyong).

This dissertation is dedicated to my family: my dear mom and my dear sister... I am very grateful for my lovely wife Wen, for her love, support and patience during these years. 


\section{Table of Contents}

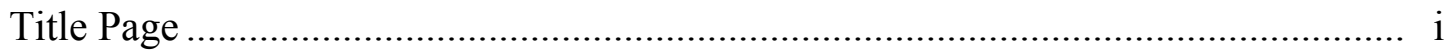

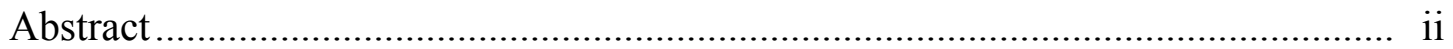

Acknowledgements .................................................................................. iii

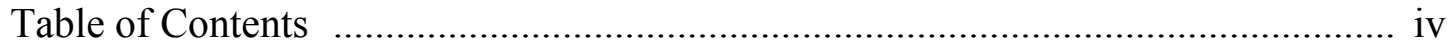

List of Figures .............................................................................................. vii

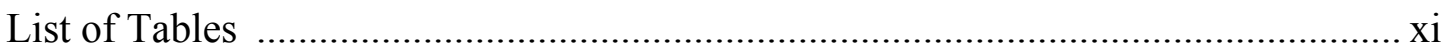

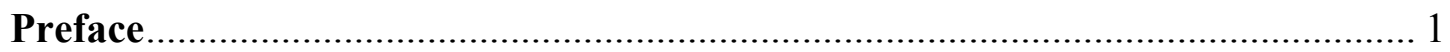

Chapter One Molecularly Imprinted Polymers (MIP) Synthesis and

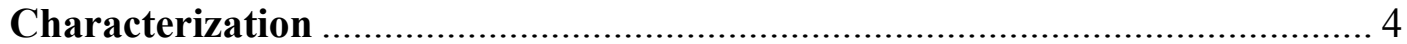

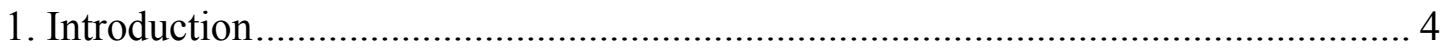

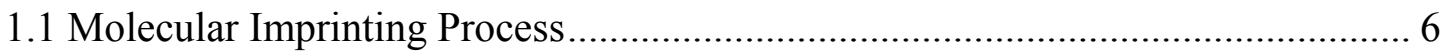

1.2. Molecular Imprinting Methodology ………………........................................ 8

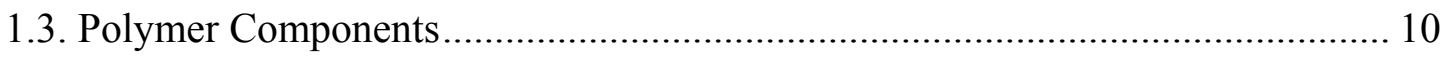

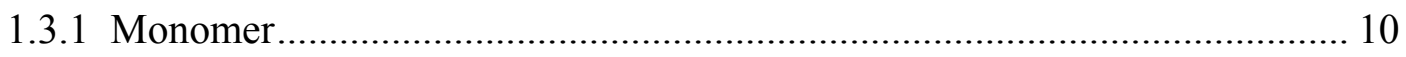

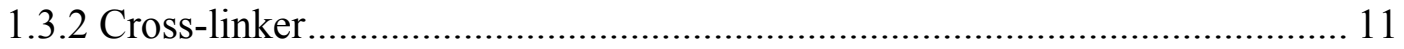

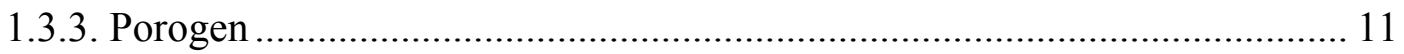

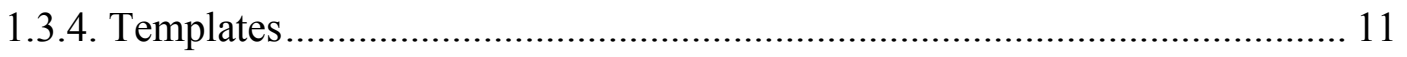

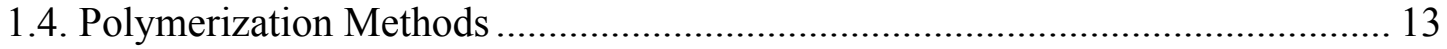

1.5. Application of Imprinted Polymers …………………..................................... 17

1.5.1. Chromatographic Separations by MIPs ……………….............................. 17

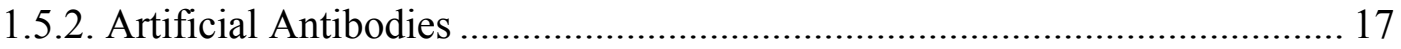

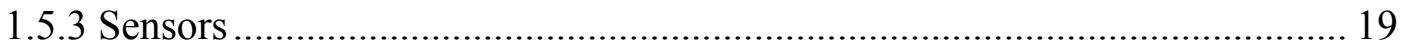

1.6 MIP Characterization by QCM Sensor ............................................................. 20

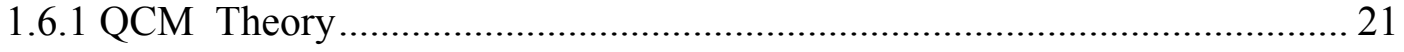

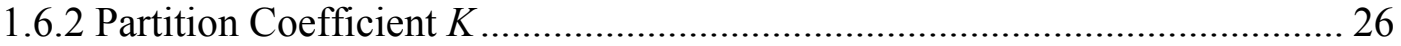

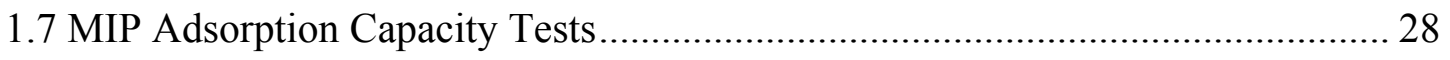

1.8. MIP Project Description ................................................................................. 33 


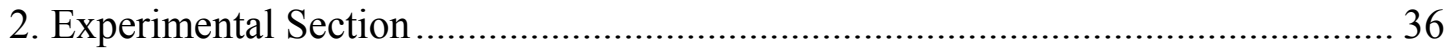

2.1 Molecular Imprinted Polymer Synthesis ……………………………………..... 36

2.1.1 Bulk Imprinted Polymer( BIP) Synthesis ……………………..................... 36

2.1.2 Imprinted Microsphere Polymer (IMP) Synthesis........................................ 37

2.1.3 Molecularly Imprinted Membrane (MIM) Synthesis .................................... 37

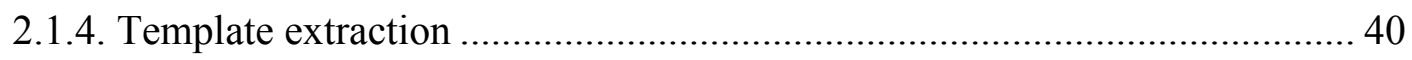

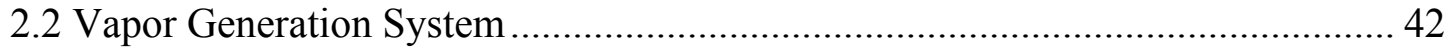

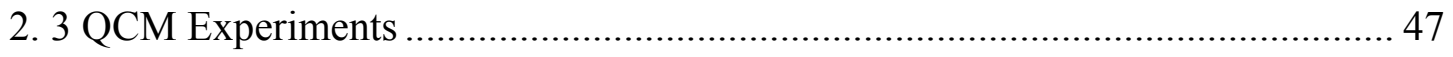

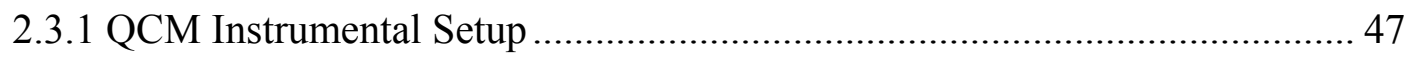

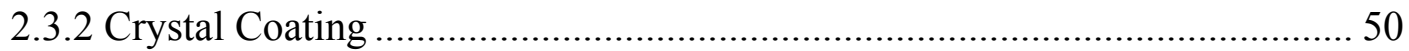

2.3.3 QCM Adsorption Measurement.................................................................... 50

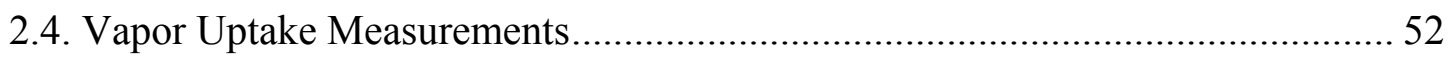

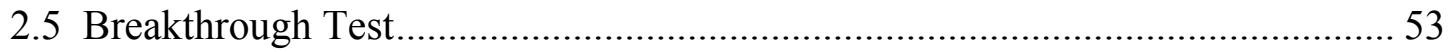

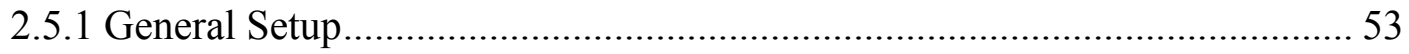

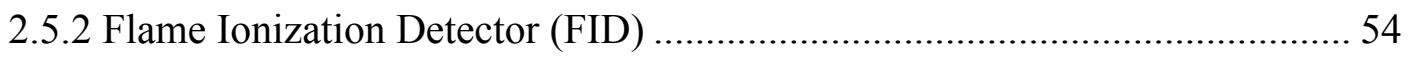

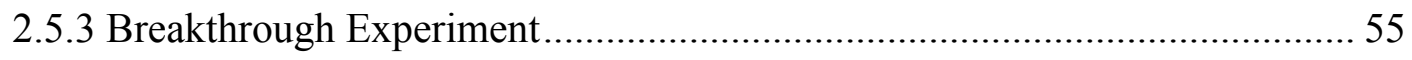

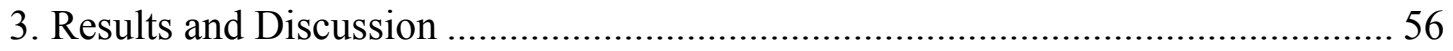

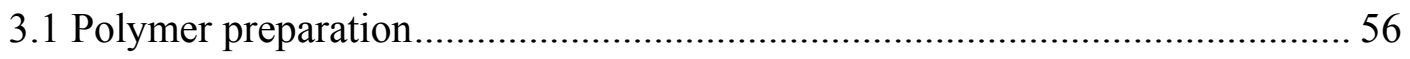

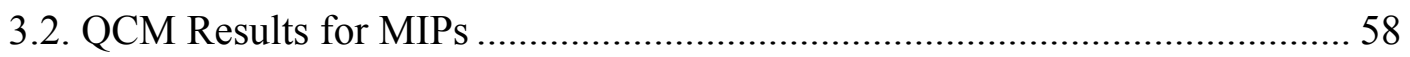

3.2.1 Formation of MIP Coatings on QCM ………............................................... 59

3.2.1.1 Molecularly imprinted membrane (MIM) coatings on QCM .................... 59

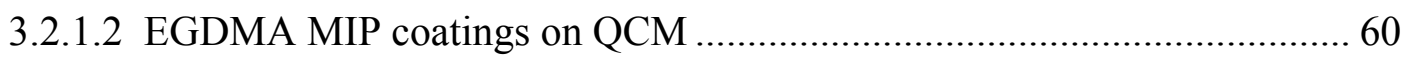

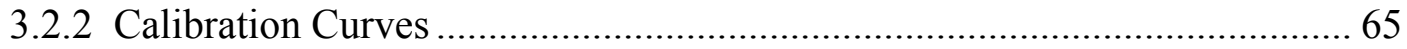

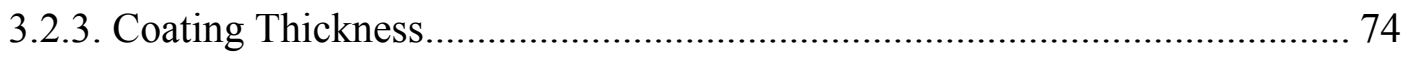

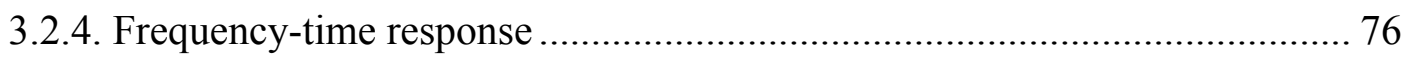

3.2.5. Comparison of Partition Coefficients …………………............................ 91

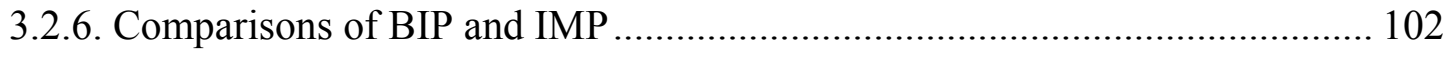

3.2.7. Influence of template concentration on the MIP performance ....................... 104

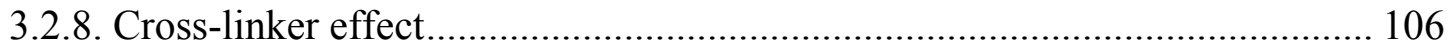

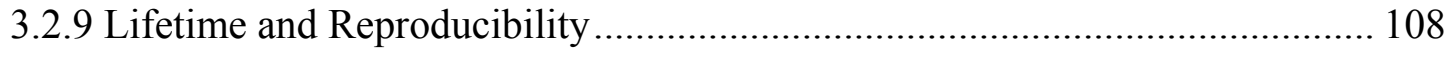




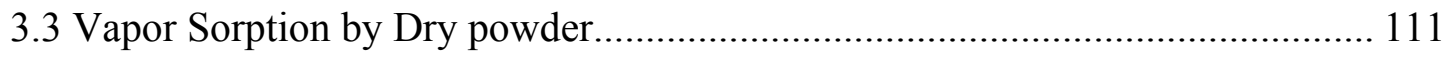

3.4. Breakthrough Studies.................................................................................... 116

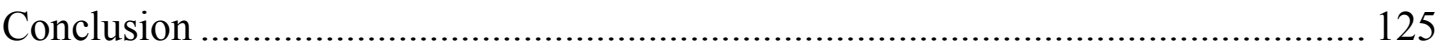

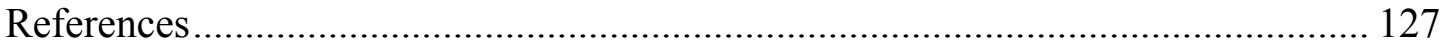

\section{Chapter Two Design, Fabrication and Characterization of Preconcentrator}

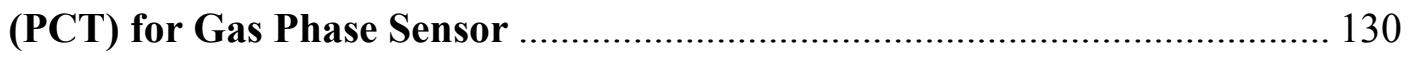

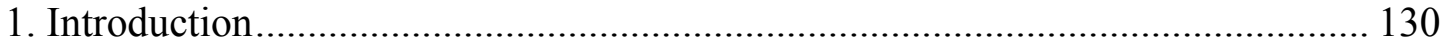

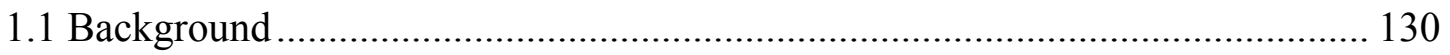

1.2 Overview of Preconcentration Methods ............................................................ 133

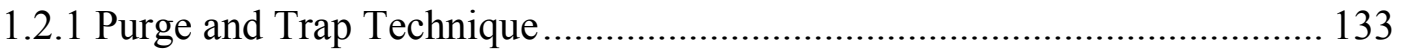

1.2.2 Solid-Phase Extraction (SPE) and Solid-Phase Microextraction (SPME) .. 135

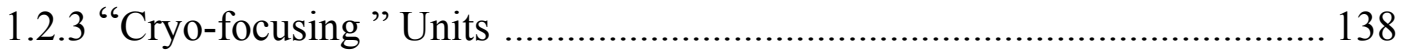

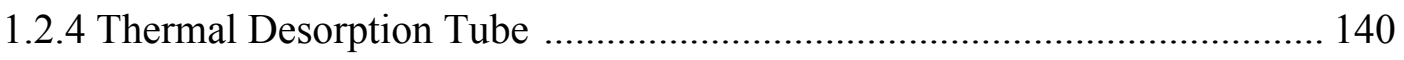

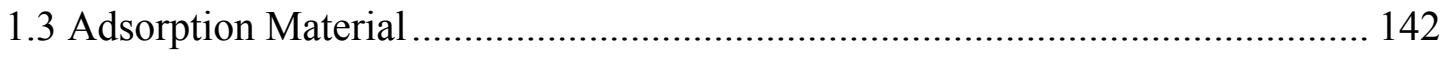

1.4 Preconcentrator Project Description ............................................................... 144

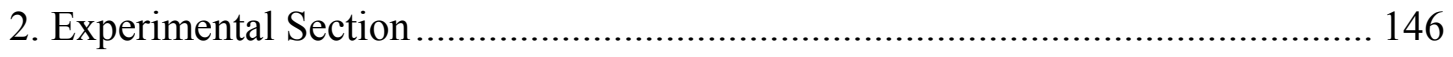

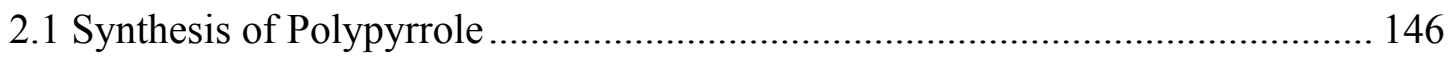

2.1.1 Polypyrrole Powder Synthesis .................................................................. 146

2.1.2 Polypyrrole layer onto Nichrome Ribbon.................................................... 147

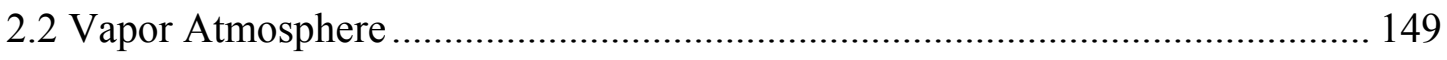

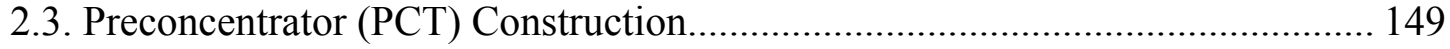

2.3.1 Preconcentrator Design I ....................................................................... 150

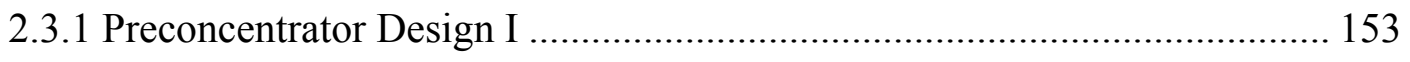

2.3.3. Preconcentrator Design III ..................................................................... 156

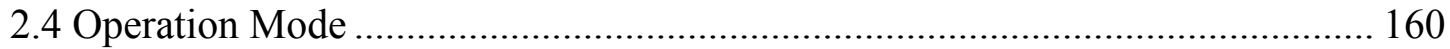

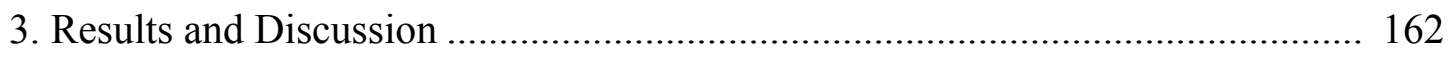

3.1. Heating characteristics .................................................................................. 162 


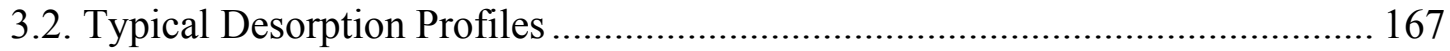

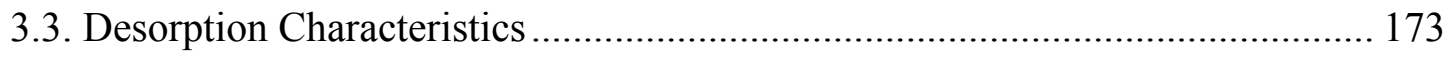

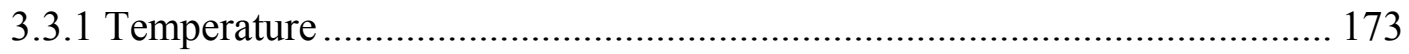

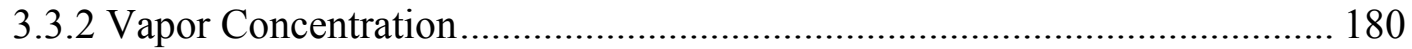

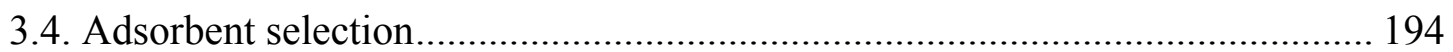

3.5 Reproducibility, Desorption efficiency and Aging Effects................................. 197

3.6. Design and Performance Modeling ............................................................... 200

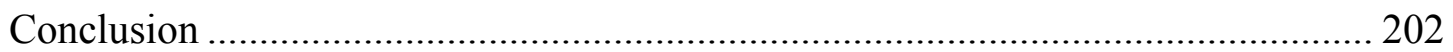

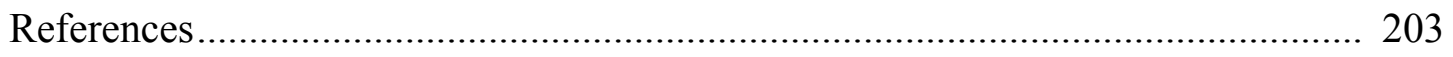




\section{List of Figures}

Figure 1.1 Principles of molecular imprinting ........................................................ 7

Figure 1.2 Common monomers for MIP production ......................................... 12

Figure 1.3 Common cross-linkers for MIP production.......................................... 12

Figure 1.4 An example of bulk synthesis method for MIP production ..................... 15

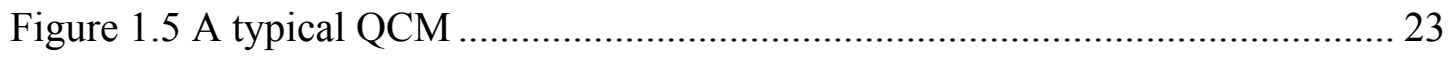

Figure 1.6 General Piezoelectric Oscillator ......................................................... 24

Figure 1.7 Equilibrium sorption of vapor into QCM coating ................................ 26

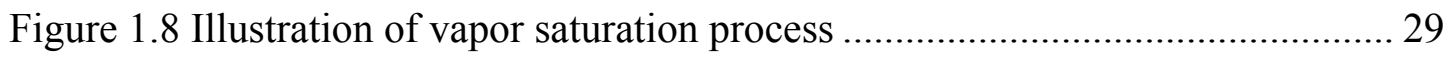

Figure 1.9 The Strategy for Creating Selective Sites within ................................... 31

Figure 1.10 Synthesis scheme of membrane imprinted polymer (MIM .................... 39

Figure 1.11 UV spectrum of template (Hydroquinone) ...................................... 41

Figure 1.12 Schematic diagram of vapor generation system ................................ 43

Figure 1.13 Heptanes emission calibration curve ............................................... 46

Figure 1.14 Schematic diagram of the apparatus for QCM .................................. 49

Figure 1.15 Breakthrough experiment set-up ................................................. 53

Figure 1.16 Calibration Curve of Toluene ............................................................ 54

Figure 1.17 Calibration curve for a PIB layer to toluene vapor............................... 63

Figure $1.1814 \mathrm{k} \mathrm{Hz}$ PIB coating on QCM response to $170 \mathrm{ppm}$.............................. 64

Figure 1.19 Block Imprinted Polymer (a) Toluene vapor....................................... 68

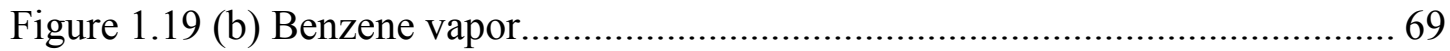

Figure 1.19 (c) Heptanes vapor.................................................................... 70

Figure 1.19 (d) Carbon tetrachloride vapor ...................................................... 71

Figure 1.20 Imprinted Microsphere Polymer (IMP) calibration curves ................... 72

Figure 1.21 Molecularly Imprinted Membrane (MIM) (imprinted ......................... 73

Figure 1.22 Frequency Response vs the coating amount....................................... 75

Figure 1.23 A typical real-time coated QCM responding ................................... 78

Figure 1.24 Response of Block imprinted polymer (BIP (HQ)) to TOL ................. 79

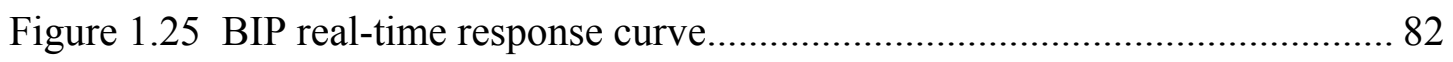


Figure 1.26. IMP real-time. 83

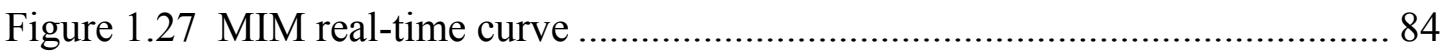

Figure 1.28 BIP coating via cellulose binder, total coating on QCM ........................ 86

Figure 1.29 Responses of imprinted and control polymer layers to toluene vapor .... 88

Figure 1.30 Responses of imprinted and control polymer layers to heptanes vapor . 89

Figure 1.31 Responses of imprinted and control polymer layers to $\mathrm{CCl}_{4}$ vapor. ......90

Figure 1.32 Selectivity pattern of bulk imprinted polymers (BIP) to organic........... 93

Figure 1.33 Selectivity pattern of imprinted micro-sphere polymers (IMP) ............ 94

Figure 1.34 Selectivity pattern of membrane imprinted polymers (MIM) ............. 95

Figure 1.35 BIP selective pattern without PIB additive layer. QCM coating............ 96

Figure 1.36 Real-time responses plot of BIP and IMP to TOL vapors................... 103

Figure 1.37 Bar figure of partition coefficients comparison of BIP(HQ) .............. 105

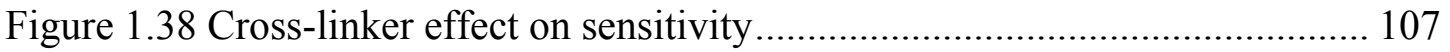

Figure 1.39 Stability of QCM coated with BIP(HQ), Test vapor ........................... 109

Figure 1.40 Linear regression plots of the breakthrough time as a function .......... 121

Figure 1.41 Adsorbent capacity as a function of vapor flow rates ........................ 123

Figure 1.42 Adsorption isotherms for BIP (HQ) and Tenax GR.......................... 124

Figure 2.1 Operating Scheme of A Preconcentrator............................................. 132

Figure 2.2 Schematic illustration of the headspace SPME method [11] ................ 137

Figure 2.3 AutoDesorb ${ }^{\circledR}$ Cryo-focusing system [13] ...................................... 139

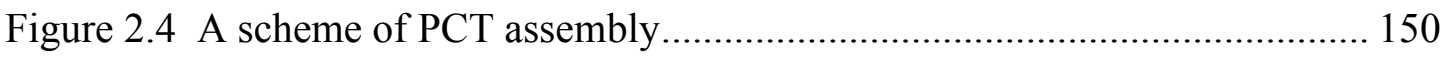

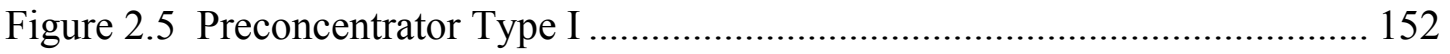

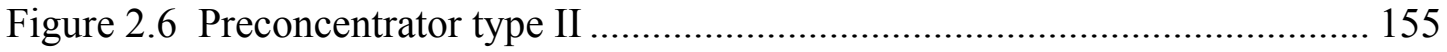

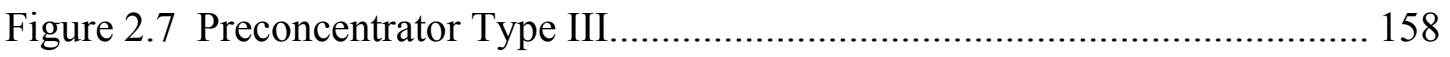

Figure 2.8 A schematic assembly of Preconcentrator Type III ............................ 159

Figure 2.9 The temperature profile of a preconcentration unit (type II)................. 164

Figure 2.10 The temperature profile of preconcentration unit (type II). ................. 165

Figure 2.11 The temperature profile of preconcentration unit (type III) ................. 166

Figure 2.12 A typical thermal desorption profile corresponding to $60 \mathrm{ppm}$........... 170

Figure 2.13 Desorption profile of Tenax GR with PCT type II............................. 171 
Figure 2.14 Desorption profile of PCT type III ................................................. 172

Figure 2.15 Thermal desorption of PPY film with PCT type II ........................... 175

Figure 2.16 Thermal desorption of Tenax GR with PCT type II............................ 176

Figure 2.17 Thermal desorption of Tenax GR with PCT type III ......................... 177

Figure 2.18Calibration curve used in PCT type II.............................................. 184

Figure 2.19 Calibration curve for toluene vapor used in PCT type III ................... 185

Figure 2.20 MIP thermal desorption profiles with respect to different vapor ......... 189

Figure 2.21 Thermal desorption profiles of Tenax GR under different ................... 190

Figure 2.22 Thermal desorption profiles of MIP under different desorption .......... 191

Figure 2.23 Desorption profiles of PPY film with different sample collecting........ 192

Figure 2.24 Desorption profiles of PPY powder (Bed packing in Type II) ............. 193

Figure 2.25 Thermal desorption toward Heptanes.............................................. 195

Figure 2.26 Thermal desorption profiles toward TOL vapor by using ................... 196

Figure 2.27 A sequential thermal desorption of PCT with PPY powder ................. 199 


\section{List of Tables}

Table 1.1 Comparison of different polymerization methods for MIP production...... 16

Table 1.2 Permeation rate data of vapors................................................................. 45

Table 1.3 Frequency changes of the quartz crystal coated with MIM (HQ) films and

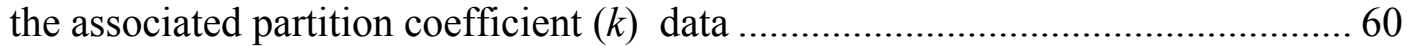

Table 1.4 Time constants of adsorption and desorption responses to vapor pulses ... 85

Table 1.5 Apparent partition coefficients (K) of PIB …....................................... 100

Table 1.6 Normalized partition coefficients of the MIPs with respect to PIB......... 100

Table 1.7 Reproducibility comparison of MIPs from different batches................. 109

Table 1.8 Vapor Uptake by Various Adsorbents............................................... 113

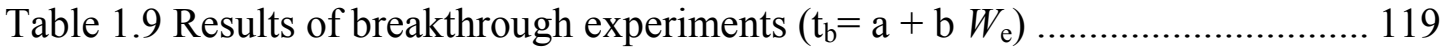

Table 1.10 Adsorption parameters derived from regression equations ................... 122

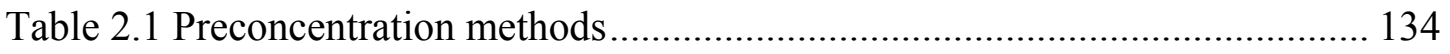

Table 2.2 Common adsorbent materials .......................................................... 143

Table 2.3 Pulses with different applied power..................................................... 178

Table 2.4 Pulses with different applied power with PCT type II........................... 179

Table 2.5 Thermal desorption of Tenax TA with PCT type II. ............................. 179

Table 2.6 Data of Preconcentration Factor and FWHM from Type II PCT ............. 186

Table 2.7 Data of Preconcentration Factor and FWHM from Type III PCT........... 188

Table 2.8 Reproducibility data of PPY film test................................................... 198

Table 2.9 Stability of Preconcentrator Design....................................................... 198 


\section{PREFACE}

The motivation for this project is the development of a field-portable chemical sensor system for the detection of volatile organic compounds (VOC's). Volatile organic compounds include compounds such as small aromatics and chlorinated hydrocarbons. To characterize VOC contamination in the field, sensors are needed to provide rapid field screening before and after remediation processes. Sensing systems are also needed for real-time, on-line monitoring of contamination levels in process during the remediation.

Most sensors being developed are based on a single device (optical, electrochemical, chemically sensitive field effect and piezoelectric) and suffer from insufficient selectivity to be functional. In order to overcome this problem, an integrated sensor array is required. We believe that such a system may contain the following elements.

1. Preconcentrator - adsorbs vapors for a fixed time interval and then releases them rapidly as a highly pre-concentrated pulse.

2. Separation system - a short gas chromatography column to partially separate the vapor pulse into its components.

3. Detector array - array of sensors with (a) rapid response times and (b) orthogonal responses to the vapors and interferences. The sensors could be based on a number of platforms (optical, electrical, piezoelectric).

4. Microprocessor - to control air flow, heating, and to analyze output of the detector array.

5. Accessories - small air pump and valves 
Each of the first three components (preconcentrator, GC column, and detector array) contributes to the overall selectivity. The stationary phase in the preconcentrator adsorbs the targeted analytes and similar compounds with high efficiency while rejecting the bulk of dissimilar compounds. Rapid heating of the stationary phase in the preconcentrator releases the adsorbed vapors as a narrow pulse. The concentration of the targeted analytes should be orders of magnitude higher in the pulse relative to the sampling air. The stationary phase in the GC column partially separates the components. The targeted analytes exit the column at a known time interval after the release from the preconcentrator. Each detector in the array possesses a unique pattern of response for the analytes and similar compounds. That response includes the amplitude and shape of the signal vs time as the pulse of analyte vapor passes through the detector. In order to tune the integrated sensor for a particular analyte or class of analytes, it is possible to work with the following variables: the identity of the stationary phase in the preconcentrator, the temperature program for the preconcentrator, the identity of the stationary phase in the GC column, the number of detectors and the selectivity of each detector. The overall selectivity of this device would be a function of the selectivity of the stationary phase in the preconcentrator, the selectivity of the stationary phase in the gas chromatography column, and the time-dependent responses of the detector array.

The work in this project is directed towards one component, the preconcentrator. In terms of its operation, the preconcentrator must exhibit a high affinity and selectivity for the targeted analytes in the presence of all other gaseous 
components. Simultaneously, the preconcentrator must be able to release the analytes rapidly on command.

The overall goal of this project is to explore molecularly imprinted polymers (MIP) as selective stationary phases for preconcentrators in gas phase sensors. Key issues are the selectivity of adsorption (selective pattern of partition coefficients), quick release, adsorption capacity and thermal stability during heating. As an initial test, the detection and quantification of small aromatic (e.g. benzene, toluene) vapors is chosen based on their molecular shapes and available vapor generation sources. Molecularly imprinted polymers are developed for the purpose of binding such planar organic vapors. Preconcentrators containing the polymer are tested for the selective adsorption of aromatic vapors in air streams and their release as a greatly enriched pulse of vapor.

This dissertation consists of two main chapters. Chapter I entitled "Molecularly Imprinted Polymers Synthesis and Characterization", discusses the synthesis protocols of various molecularly imprinted polymers and their characterization by Quartz Crystal Microbalance (QCM) Sensors, vapor uptake weight measurements and breakthrough studies. Chapter II entitled " Design, Fabrication and Characterization of Preconcentrators (PCTs) for Gas Phase Sensors", discusses the preconcentrator design and the application of different adsorbents in preconcentrators. 


\section{CHAPTER ONE}

\section{Molecularly Imprinted Polymers (MIP) Synthesis and Characterization}

\section{Introduction}

The sensitive and selective determination of a large number of trace organic compounds is of great relevance in many fields, such as food industry, environment, airport transportation, biotechnology and health care for the diagnosis of disease. In recent years, various sensors have been developed to detect low concentrations of air pollutants which are frequently encountered in every day life [1]. Selective response is one of the most crucial properties of a sensor, particularly at low analyte concentrations in the presence of interfering substances.

Living beings are capable of recognizing chemical changes in their metabolic states and in their environment with high selectivity and sensitivity by using specific receptors [2]. These interactions rely on selective binding between the receptors and their ligands. The binding interactions are based on the delicate recognition properties such as those exhibited by antibodies and enzymes, which can distinguish between their specific target analytes and other structurally related compounds. The outstanding specificity and sensitivity of these biological receptors make them highly attractive for the development of sensors. However, devices that rely on biological molecular recognition elements often lack storage and operational stability. Thus, the 
tedious procedure and cost of biological molecules have led to attempts to synthesize antibody mimics in the chemistry laboratory.

An emerging technology called molecular imprinting could provide an alternative approach to create receptor-like binding sites. In this technology, complexes between imprinting molecules (templates) and functional monomers are allowed to self-assemble in solution and, subsequently, the three-dimensional structure of these complexes is formed by polymerization with a high degree of crosslinking [3]. The highly cross-linked polymeric nature of such imprinted matrices leads to a strong tolerance towards various external conditions, such as exposure to acidic, basic and organic solvents. In addition, these highly stable synthetic polymers possess selective molecular recognition properties because of recognition sites inside the polymer matrix that are complementary to the analyte in the shape, size and orientation of functional groups. Some of these polymers have high selectivity and affinity constant, comparable with naturally occurring recognition systems such as monoclonal antibodies or receptors [4], which make them especially suitable as recognition elements in chemical sensors for analytical chemistry. Thus, molecularly imprinted polymers (MIP) have been widely used in a range of applications requiring selective ligand binding, such as solid phase adsorbents in HPLC for the separation and analysis of racemic mixtures [5]. 


\subsection{Molecular Imprinting Process}

The development of MIPs was pioneered by Klaus Mosbach and co-workers [6]. They mainly used methacrylic acid, which has a carboxyl group, as the functional monomer. Methacrylic acid was mixed with a template molecule and copolymerized with an excess of a cross-linking agent like ethylene glycol dimethacrylate.

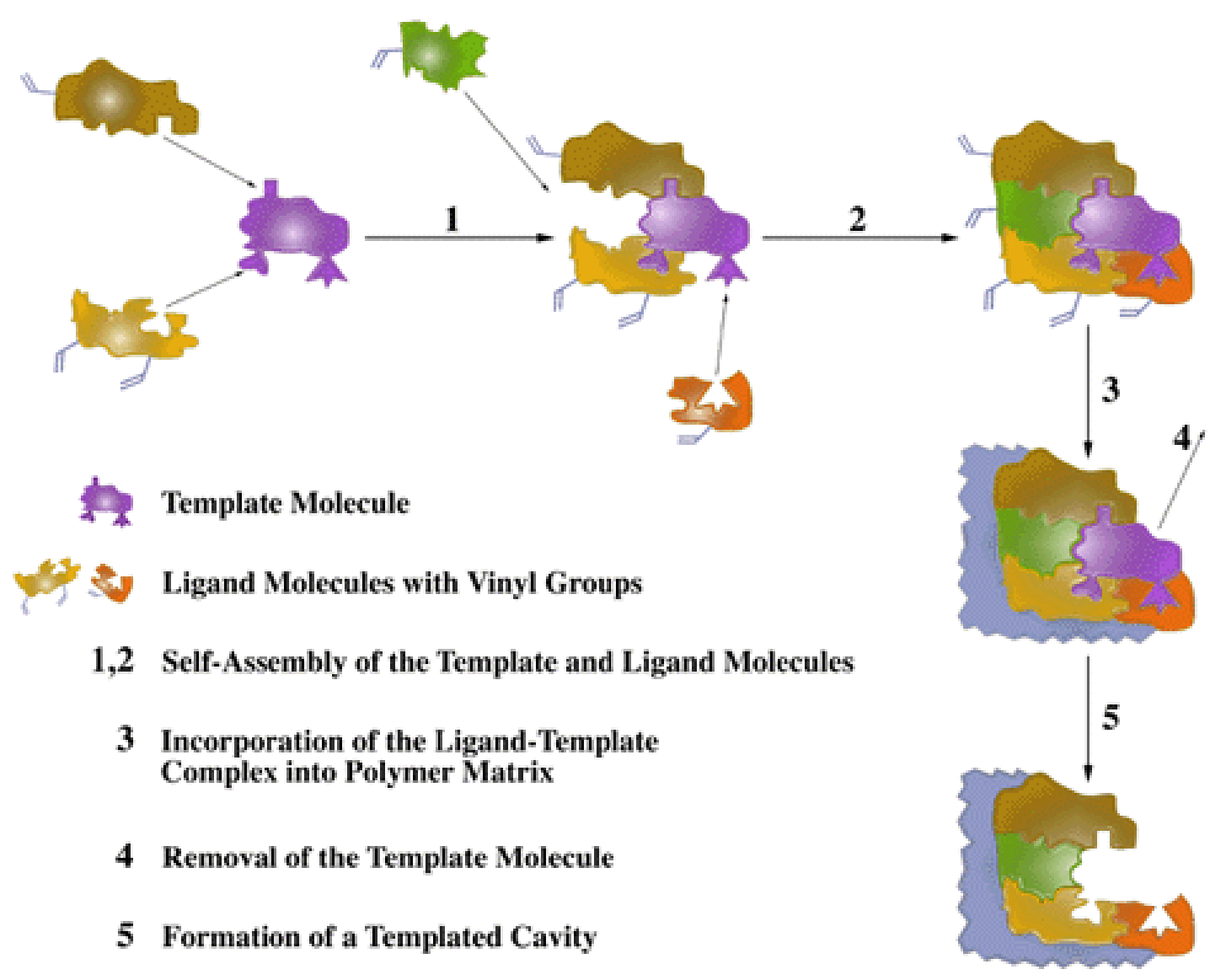

Figure 1.1 Principles of molecular imprinting (Figure source: reference [7])

In the first step of generating a MIP (Figure 1.1), the template molecule is mixed with a functional monomer. Functional monomers are single chemical units 
that finally react to form the polymer. These monomers have specific chemical functionalities such as carboxyl, hydroxyl, amino or aromatic groups which can bind to the template molecule covalently or non-covalently [8]. The selection of the specific functional monomer, or the combination of monomers depends on the particular chemical properties of the analyte for which the MIP is being designed.

Once these components are allowed to mix and associate with one another, the assembly is polymerized using excess cross-linking agent. This gives a highly crosslinked, rigid, glassy polymer with functional groups "fixed" in a specific orientation around the template molecule. The embedded template is then chemically cleaved (for example, hydrolyzed) or extracted from the polymer, depending on whether it is bound covalently, non-covalently or both.

When the template molecule is removed, a rigid "imprint' of the molecule is left inside the polymer matrix. In most applications, the polymer is ground into fine particles, thereby increasing the surface area available for interaction with the analyte. 


\subsection{Molecular Imprinting Methodology}

Molecular imprinting can be achieved by two approaches, via covalent and non-covalent interactions. Covalent imprinting is completed by a covalent interaction between a functional monomer and a template. This complex is subsequently incorporated into the polymer matrix during the polymerization. After the polymer is formed, the imprint template is extracted by cleavage of the covalent bonds, usually by acid hydrolysis. These covalent bonds must be easily cleavable. Template molecules without suitable functional groups have to be derivatized for a linkage. The resulting sites are therefore obtained with an induced process of pre-organization.

The other approach solely uses non-covalent interactions in the recognition of the imprint species. Low-weight organic molecules without distinct functional groups, such as organic solvents or anesthetics, are candidates for non-covalent imprinting [9]. Typical interaction types that have been exploited are ionic interactions [10 ], hydrogen bonding [11], and hydrophobic interactions [12]. When these normally weak interactions have been established in solution, polymerization is initiated and a molecular matrix is formed around the imprinting species. After polymer formation, the template molecules can be dissociated from the polymer matrix by mild extraction procedures [13] . The formed 3-D architecture inside the matrix is thus complementary to the shape and size of the templates. The target analytes simply diffuse into and out of the polymer matrix.

Compared to the covalent imprinting method, the major advantages of noncovalent imprinting are the versatility in the range of size, shape and functionality of the template molecule, and the time-saving synthesis. When working with non- 
covalent imprinting, it is preferable to use lower polymerization temperatures; higher temperatures will result in weaker monomer/template complexes[14]. In both cases, the procedure of imprinting depends highly on successful extraction of the templates. 


\subsection{Polymer Components}

\subsubsection{Monomer}

The functional monomer used for producing a useful MIP must strongly interact with the template to achieve a high yield of imprinted binding sites and allow the maximum number of complementary interactions to be developed in the polymer

matrix. In general, analytes containing basic functional groups are best imprinted with monomers containing acidic functional groups and vice versa.

Some common monomers used are shown in Figure 1.2. Methacrylic acid is one of the most widely used monomers. It interacts ionically with amine functional group and via hydrogen bonding with a variety of polar functional groups such as alcohols, carboxylic acids, carbamates and carboxylic esters.

\subsubsection{Cross-linker}

The enhancement of selectivity in MIPs is mainly due to the rigidity of the imprint cavities. In order to achieve a high specificity and selectivity for the polymer, a high degree of cross-linking is used in the imprinting process. Ethylene glycol dimethacrylate (EGDMA) has been used widely as a cross-linker in noncovalent molecular imprinting (Figure 1.3). New cross-linkers, which can be tri- or tetrafunctional, have been studied in some detail [15]. These include divinylbenzene (DVB), trimethylpropanetrimethylacrylate (TRIM), and pentaerythritol tetracrylate (PETEA) (Figure 1.3) . Molecularly imprinted polymer with high loading capacity 
and excellent resolving capability were obtained using TRIM when compared with EGDMA polymer [15].

\subsubsection{Porogen}

The choice of porogenic solvent is critical in most molecular imprinting procedures. The porogen solvent is a chemically inert agent which promotes channel formation in the highly cross-linked polymer. The porogen used in the imprinting step is likely to influence the strength of the interaction between the template molecule and polymerizing matrix. The solvent used in the polymer formation should be as non-polar as possible in order to maximize the strength of hydrogen-bonding and ionic interactions between the template molecular and the monomer.

\subsubsection{Templates}

The strength of binding affinity of the MIPs to analytes depends on the number and type of interaction sites, the template shape and the imprint cavity rigidity. Templates offering multiple interactions sites for the functional monomer are likely to yield binding cavities with higher specificity and affinity for the template. Templates possessing conformational rigidity can fit well in the cavity of the polymer matrix with minimal change in conformation and will increase the affinity and selectivity in the recognition [16-17].

In addition to the type of template, the ratio of the template to the functional monomer has been known to play a key role in the selectivity and sensitivity in the imprinted polymers . The optimum ratio has to be determined for each individual template [18]. 
<smiles>C=CC(=O)O</smiles>

Acrylic acid<smiles>C=C(C)C(=O)O</smiles>

Methacrylic acid<smiles>C=CC(=O)OC</smiles>

Methyl methacrylate
$\mathrm{H}_{2} \mathrm{C}=\mathrm{CHCH}_{2} \mathrm{NH}_{2}$

Allylamine
$\mathrm{CH}_{2}=\underset{\mathrm{I}}{\mathrm{C}} \mathrm{H}$

Acrylonitrile

Figure 1.2 Common monomers for MIP production<smiles>C=C(C)C(=O)OCCOC(=O)C(=C)C</smiles>

Ethylene Glycol Dimethacrylate<smiles>C=Cc1ccc(C=C)cc1</smiles>

Divinylbenzene<smiles>C=C(C)C(=O)OCC(CC)(COC(=O)C(=C)C)COC(=O)C(=C)C</smiles>

Trimethylopropane

Trimethacrylate

Figure 1.3 Common cross-linkers for MIP production 


\subsection{Polymerization Methods}

A variety of techniques have emerged during the last few years which use a range of strategies to synthesize imprinted polymers. These methods are summarized in Table 1.1.

Traditionally, molecular imprinting has mostly been performed by bulk polymerization (Figure 1.4), in which functional monomers are bound either covalently or non-covalently to a template molecule. The monomer mixture is then copolymerized with a cross-linker in the presence of a porogen and a free radical initiator. Polymerization of this mixture results in a highly cross-linked insoluble polymer. Removal of the template leaves sites complementary in size and shape to the template molecule. The block of polymer is then ground and sieved in order to produce particles of an appropriate size for subsequent experiments. This process is time-consuming and wasteful since the polymer has to be sedimented several times to define particle size before the material is ready to be packed into HPLC columns or otherwise used.

To date, there is a wide range of techniques for preparing MIP beads, ranging from the use of pre-formed beads to suspension polymerization in water or organic solvents or dispersion polymerization [19]. These protocols were introduced a few years ago, and this area is still under investigation. The bead-shaped material is being developed mainly for use in packed column chromatography. This technique would be of potential interest for various analytical applications.

More recently, a new class of the membranes with imprinting functionality has been reported [20]. The investigations have concentrated on the imprinting 
properties of the micro-porous membrane poly(acrylonitrile-co-acrylic acid) [21]. This copolymer contains both acrylic acid residues as the functional sites and acrylonitrile (ACN) residues as the membrane formation sites. Membranes of the imprinted matrix are formed by the phase inversion precipitation method. Phase inversion is a process where a polymer is transformed in a controlled manner from a solution to a solid state. In particular, immersion precipitation process is commonly used to fabricate membranes. By controlling the initial stage of immersion precipitation process, the membrane morphology can be controlled, i.e. finger-like and sponge-like membranes can be prepared. In this method, the copolymer matrix and the template molecule are dissolved simultaneously in solution, allowing the imprinting process to be completed in solution. The thin film is precipitated in a solvent such as water. The resulting polymer membrane contains some imprint sites of the template after washing the template away with water. These approaches present the possibility of preparation of molecular imprinted membrane for selective separation. 

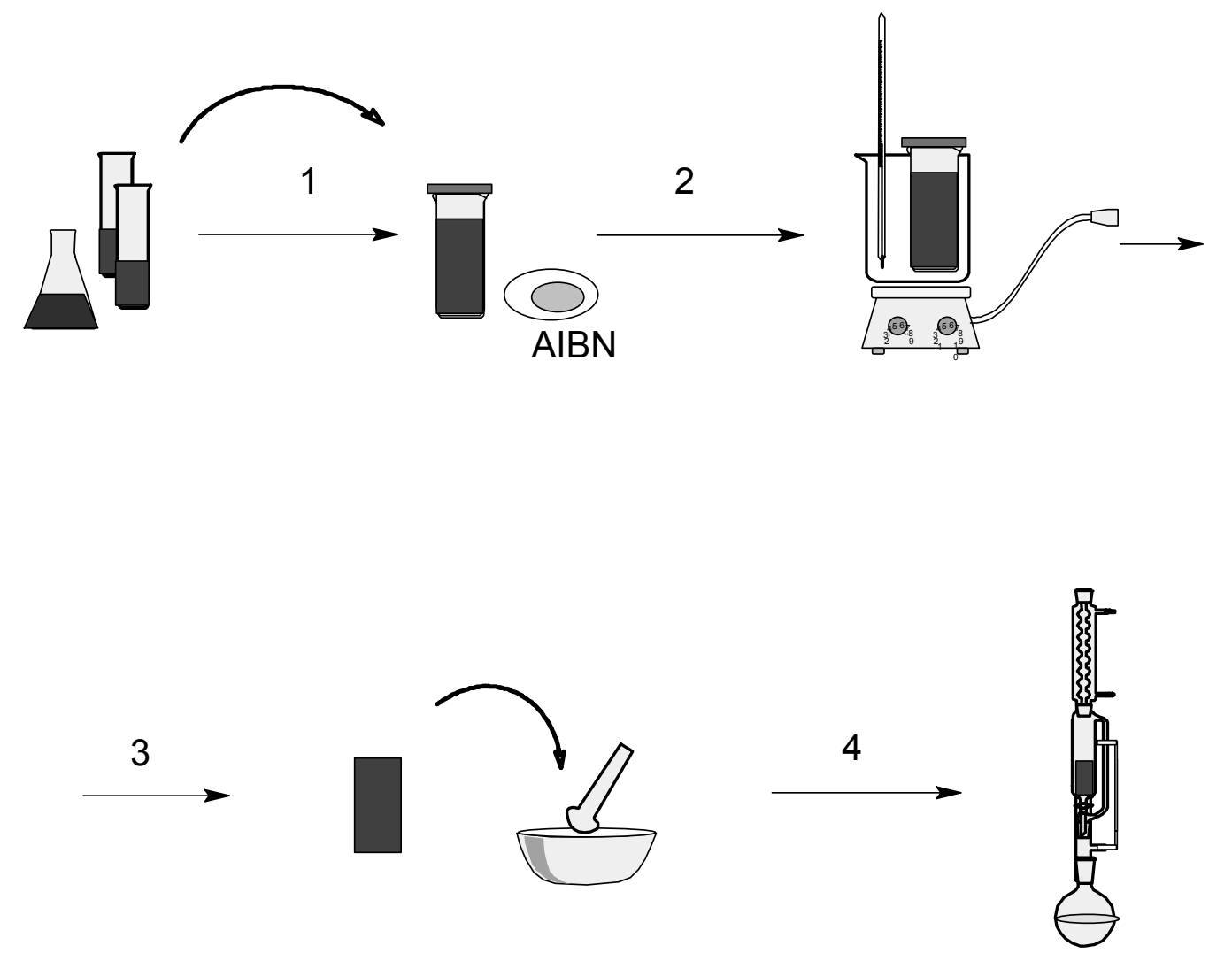

Figure 1.4 An example of bulk synthesis method for MIP production. (1) Dissolution of monomer and template in a solvent. (2) Free radical polymerization initiated with an azo initiator and a cross-linker agent in a water bath. (3) Crushing the block polymer into fine particles. (4) Removal of template from the polymer matrix using Soxhlet apparatus. 
Table 1.1 Comparison of different polymerization methods for MIP production

\begin{tabular}{|c|c|c|c|c|}
\hline $\begin{array}{l}\text { Polymerization } \\
\text { Method }\end{array}$ & $\begin{array}{l}\text { Operating } \\
\text { Complexity }\end{array}$ & Product & Advantage & Disadvantage \\
\hline Bulk- in block [22] & low & $\begin{array}{l}\text { Random } \\
\text { fragments } \\
\text { after } \\
\text { grinding }\end{array}$ & $\begin{array}{l}\text { Simplicity, } \\
\text { imprinting not } \\
\text { affected by } \\
\text { method derived } \\
\text { factors. Large } \\
\text { literature }\end{array}$ & $\begin{array}{l}\text { Tedious } \\
\text { processing. }\end{array}$ \\
\hline $\begin{array}{l}\text { Suspension- in } \\
\text { perfluorocarbon [23] }\end{array}$ & High & $\begin{array}{l}\text { Spherical } \\
\text { beads }\end{array}$ & $\begin{array}{l}\text { Good quality } \\
\text { beads produced }\end{array}$ & $\begin{array}{l}\text { Expense of } \\
\text { liquid } \\
\text { fluorocarbon, } \\
\text { complicated } \\
\text { experimental } \\
\text { condition } \\
\text { required, little } \\
\text { literature or } \\
\text { optimization to } \\
\text { date }\end{array}$ \\
\hline $\begin{array}{l}\text { Phase inverse } \\
\text { precipitation [20] }\end{array}$ & Medium & Thin film & $\begin{array}{l}\text { Cheap, easy to } \\
\text { cast }\end{array}$ & $\begin{array}{l}\text { Solvent } \\
\text { wasteful, } \\
\text { tedious } \\
\text { processing }\end{array}$ \\
\hline Microsphere [24] & Medium & $\begin{array}{l}\text { Microsphere } \\
\text { about } 10 \text { um }\end{array}$ & $\begin{array}{l}\text { Uniform size, } \\
\text { high yield, }\end{array}$ & $\begin{array}{l}\text { Low binding } \\
\text { affinity }\end{array}$ \\
\hline $\begin{array}{l}\text { Suspension - in water } \\
{[25]}\end{array}$ & $\begin{array}{l}\text { Medium - } \\
\text { high }\end{array}$ & $\begin{array}{l}\text { Spherical } \\
\text { beads }\end{array}$ & $\begin{array}{l}\text { Highly } \\
\text { developed } \\
\text { technology, } \\
\text { high quality, } \\
\text { reproducible }\end{array}$ & $\begin{array}{l}\text { Water is } \\
\text { incompatible } \\
\text { with most } \\
\text { imprinting } \\
\text { procedures, only } \\
\text { possible for } \\
\text { some covalent } \\
\text { and metal } \\
\text { chelate based } \\
\text { processes }\end{array}$ \\
\hline
\end{tabular}




\subsection{Application of Imprinted Polymers}

Since the initial work by Wulff's group in the early 1970s on the development of MIPs for sugar and amino acid derivatives, MIPs for over 20 classes of compounds have been reported for various practical applications [26]. The list includes sugars, amino acids, peptides and proteins, therapeutic drugs, steroids, metal ions, aromatic hydrocarbons, dyes and pesticides.

\subsubsection{Chromatographic Separations by MIPs}

Molecularly imprinted polymers have been widely used as stationary phases for separation by high performance liquid chromatography (HPLC). Most work has concentrated on the resolution of chiral compounds [27].

Mosbach and his coworkers have successfully developed a synthetic polymer selective for (S)-naproxen by molecular imprinting [28]. EGDMA and 4vinylpyridine were copolymerized in the presence of the template, $(\mathrm{S})$ - naproxen. (R,S)-Naproxen was well resolved on this chiral separation phase. A separation factor of 1.65 and a resolution factor of 0.83 were obtained when $2 \mu \mathrm{g}$ of the racemate was loaded on the column. In addition, this imprinted polymer was able to separate naproxen from the structurally related ibuprofen and ketoprofen.

\subsubsection{Artificial Antibodies}

Another area for which MIP have been tested is their suitability as antibody mimics. Polymers prepared against theophylline or diazepam were used as antibody substitutes for competitive binding assays to quantitate theophylline in serum samples [6]. This method relies on the inhibition of radio-labeled ligand binding by the serum 
analyte. The amount of labeled ligand bound to the polymer is inversely related to the concentration of drug in the sample. The sorbent assay based on molecularly imprinted polymers for theophylline, was linear over the range of $14-224 \mu \mathrm{M}$, which is satisfactory for therapeutic monitoring of the drug. The assay responded to diazepam linearly over the range of $0.44-28 \mu \mathrm{M}$. The specificity of this type of polymer was also tested by the determination of cross-reactivity (competitive binding) of drugs structurally related to theophylline or diazepam. The cross-reactivity profiles of these MIPs were practically identical to those reported for antibodies against these drugs. The accuracy of the MIP assay measurement of theophylline was evaluated by analysis of 32 patient serum samples, and the results were in excellent agreement with values determined using a commercial immunoassay for theophylline. Preparations of MIPs against proteins, DNAs and other biomolecules is still under investigation. Nevertheless, the stability and the ability of MIPs to operate in extreme conditions, in particular in organic solvents, is attractive to analytical applications. 


\subsubsection{Sensors}

One of the most attractive applications developed in the molecularly imprinting area is the use of MIP as the recognition elements in sensor devices. MIPs have unique properties that make them especially suitable for sensor technology. Besides exhibiting good specificity for various compounds of medical, environmental, and industrial interest, MIPs have excellent operational stability. Their recognition properties are unaffected by acid, base, heat or organic solvent treatment $[6,9,10]$. During the past few years, mass-sensitive acoustic transducers, such as the surface acoustic wave oscillator (SAW) or the quartz crystal microbalance (QCM) have become increasingly popular for the design of polymer-based sensors [29]. A novel MIP sensor based on QCM was designed by $\mathrm{Li}$ and his coworkers to provide enantioselectivity for dansylphenylalanine enantiomers [30]. Chiral dansyl-Lphenylalanine was used as the template for the polymer imprinting. The imprinted polymer was coated onto QCM sensor with a photoactive precursor. The sensor was used to distinguish L-and D-dansylphenylalanine enantiomers in solution. The designed sensor could determine the enantiometric composition of L-and Denantiomeric mixtures quantitatively over a wide range from 5 to $500 \mu \mathrm{g} / \mathrm{mL}$ at $\mathrm{pH} 10$. The detection limit for the reported sensor is $5 \mu \mathrm{g} / \mathrm{mL}$.

Karube and co-workers have been working on a novel type of piezoelectric odor sensors based on molecularly imprinting technology [31]. In their work, piezoelectric quartz crystals coated with a 2-methylisoborneol-imprinted polymer gave responses which were consistently $5-10 \mathrm{~Hz}$ (1.1-1.3 times) higher than those 
sensors coated with a non-imprinted polymer in the presence of 2-methylisoborneol vapor. A number of other odorants were examined and the responses of the nonimprinted sensors were found to be similar to or greater than the response of the imprinted sensors. As reported, the sensor had a detection limit of ca. $5 \mathrm{mg} / \mathrm{L}$. To the best of our knowledge, this is the only preceding report of the use of MIPs on QCMs for the selective detection of a vapor.

So far, MIP sensors have not made their way to commercial applications. However, the results mentioned here and the current effort in molecular imprinting with small organic molecules suggest a promising future.

\subsection{MIP Characterization by QCM Sensors}

Mass-sensitive chemical sensors, such as quartz crystal microbalances (QCMs), are often used for the detection and monitoring of volatile organic compounds and other analytes. A QCM oscillates at a characteristic resonant frequency when placed in a feedback circuit. Usually, the QCM surface is coated with a thin film of stationary phase that is capable of adsorbing the analytes. Vapor sorption increase the mass at the surface of the QCM device and results in a shift in the oscillator frequency. Thus, the QCM device represents a sensitive probe for characterizing thin films and for investigating interaction mechanisms between chemical vapors and coating materials.

The advantages of using QCM sensors to evaluate selective vapor adsorption are their high detection sensitivity of less than $1 \mathrm{ng} / \mathrm{cm}^{2}$ and their rapid response times when performing experiments [32]. An additional advantage is the potential for these 
sensors to be adapted to a variety of gas-phase analytical problems by designing or selecting specific coating for particular applications. Since the first report by King [33], the use of piezoelectric quartz crystals for the detection of gaseous compounds has become increasingly popular [33-40]

\subsubsection{QCM Theory}

As a piezoelectric material, the quartz wafer exhibits the piezoelectric effect (piezoelectricity), which is defined as the production of electrical charges by the application of mechanical stress [32]. The phenomenon is reciprocal. Applying an appropriate electrical field to a piezoelectric material creates a mechanical stress. An $\mathrm{AC}$ voltage near a mechanical response frequency results in a vibration or oscillation of the crystal and forms an acoustic wave spread through or across the surface of the crystal. Any changes to the characteristics of the acoustic path affect the velocity and/or amplitude of the wave. The velocity changes can be monitored by measuring the frequency or phase characteristics of the sensor and can then be correlated to the corresponding physical quantity being measured.

The most commonly used piezoelectric sensors are the thickness-shear mode (TSM) acoustic sensor, (also known as quartz crystal microbalance (QCM)), the surface acoustic wave (SAW) device and the shear-horizontal acoustic plate mode (SH-APM) device [35].

A quartz crystal microbalance is a small, thin disk of AT-cut quartz with parallel circular metal electrodes bonded on both sides to make electrical connections (Figure 1.5 a). An AT-cut crystal is a thin piece of quartz with two parallel or slightly 
convex surfaces, usually about the size of a nickel or less [33]. The metal electrodes are used to induce an oscillating electric field perpendicular to the surface of the wafer. The application of a voltage causes the AT crystal to move sideways internally in a thickness shear movement, as shown in Figure $1.5 \mathrm{~b}$. 


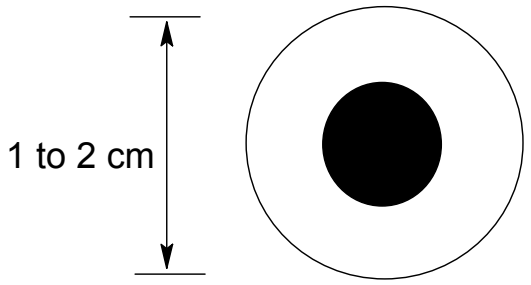

Top face of a QCM, black disc is a metal electrode

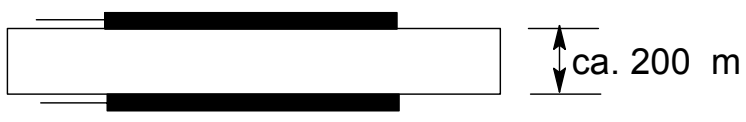

Side view of a QCM showing metal electrodes

(a)

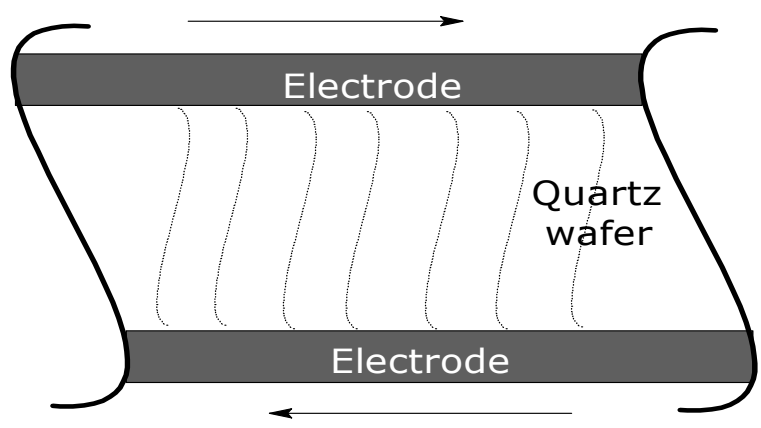

Shearing mode of vibration

(b)

Figure 1.5 A typical QCM .

(a) Orientation of electrodes

(b) Shear deformation during oscillation at the fundamental frequency 
In order to obtain frequency information from a QCM, the crystal wafer becomes a feedback element of the amplifier (Figure 1.6). In this design, the whole circuit oscillates at a characteristic fundamental frequency. Depending on the thickness of the wafer, this fundamental frequency is usually between 2 and $20 \mathrm{MHz}$.

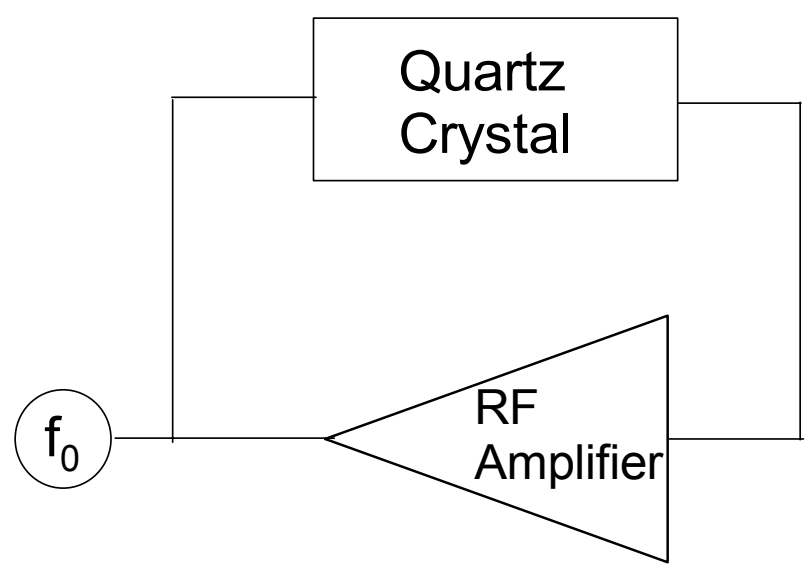

Figure 1. 6 General Piezoelectric Oscillator

The frequency of this coupled mechanical and electrical oscillation in the QCM depends on several factors. Factors that are normally constant include the physical properties of the quartz wafer (thickness, density and shear modulus). Factors that often change are the mass of the attached electrode or the mass of a coating film attached to that electrode. These interface mass changes are related in a simple manner to changes in the QCM oscillation frequency according to the Sauerbrey equation [32]. 


$$
\Delta \mathrm{f}=k \mathrm{~F}^{2}(\Delta \mathrm{m} / \mathrm{A})
$$

Where $\Delta \mathrm{f}$ is the change in frequency due to the mass loading, $k$, with an approximate value of $-2.3 \times 10^{6}$ [32], is the crystal constant based on the physical properties of the crystal (the crystal shape, electrode geometry and placement), $\mathrm{F}$ is the fundamental frequency $(\mathrm{MHz}), \Delta \mathrm{m}$ is the change of mass on the crystal surface $(\mathrm{g})$, and $\mathrm{A}$ is the active area of the crystal $\left(\mathrm{cm}^{2}\right)$.

This equation describes the relationship between the deposited mass on quartz crystal and the corresponding change in resonant frequency of the crystal. The frequency of QCM decreases as the loading mass on the crystal increases. This equation also predicts that a commercially available $10 \mathrm{MHz}$ QCM would have a mass sensitivity of about $400 \mathrm{~Hz} \cdot \mathrm{cm}^{2} / \mu \mathrm{g}[32]$. 


\subsubsection{Partition Coefficient $K$}

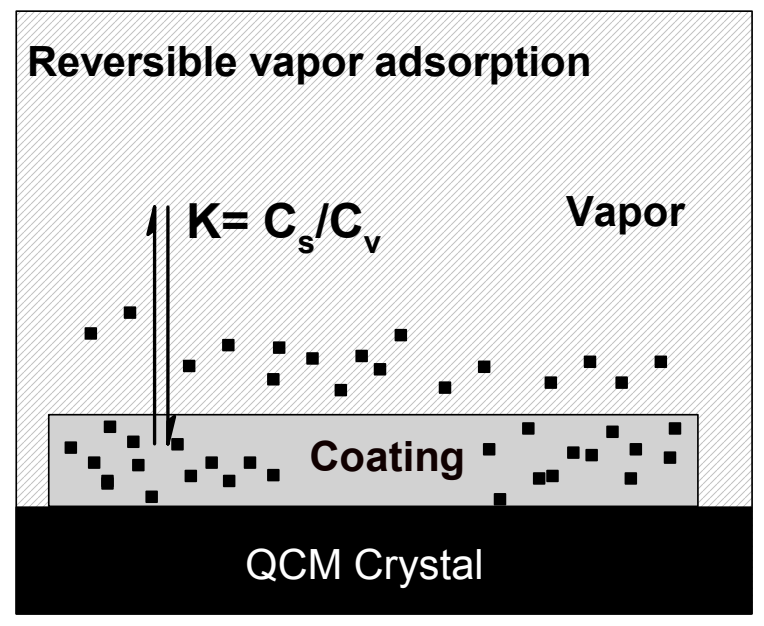

Figure 1.7 Equilibrium sorption of vapor into QCM coating

Equilibrium sorption of ambient vapor into the QCM device coating

represents a partitioning of the solute vapor between the gas phase and the stationary phase. This process is illustrated in Figure 1.7.

The distribution can be quantified by a partition coefficient, $K$, a useful index of the sorption characteristics of a sensing film. Thermodynamically, $\mathrm{K}$ is a measurement of the equilibrium distribution of solute molecules between the gas phase and the stationary phase. In a steady state, the amount of sorbed gas molecules onto/into the sensing film is determined from the interaction between gas molecules and film material. This value is independent of the kind of devices, resonance frequency and so on, and is constant in the range of low gas concentration, where Henry's law is valid. $\mathrm{K}$ is defined by [41]: 


$$
K=C_{S} / C_{V}
$$

Where $C_{V}$ is gas concentration in the air, and $C_{S}$ is that in the film. Substitution of this equation into the general form of the Sauerbrey equation then leads to the following equation [40]:

$$
K=\Delta f_{v} \rho / \Delta f_{s} C_{V}
$$

where $\Delta f_{\mathrm{v}}$ is the frequency shift due to the vapor adsorption, $\Delta f_{\mathrm{s}}$ is the frequency shift due to the deposition of film itself, $\mathrm{C}_{\mathrm{v}}$ is the vapor concentration and $\rho$ is the density of the film (assumed to be approximately $1 \mathrm{~g} / \mathrm{cm}^{3}$ for the polymers in this research). This equation assumes that all frequency changes are due to changes in the mass of the film. A linear calibration curve of $\Delta f_{\mathrm{v}}$ vs $\mathrm{C}_{\mathrm{v}}$ yields an apparent value of partition coefficient $\mathrm{K}$ from its slope.

The polymer coatings for QCM can be evaluated based upon the relative magnitude of the partition coefficients of the targeted vapors with respect to control polymers or with regard to possible interfering compounds. 


\subsection{MIP Adsorption Capacity Tests}

In order to assess MIPs as adsorbents for collecting organic vapors in preconcentrator, some data on the adsorption capacity are needed. The vapor adsorption capacity is the maximum mass of adsorbed species per mass of adsorbent. For a given adsorbent, adsorption capacity can be used for determining and comparing the amount of various compounds being retained. Thus, it is a major performance parameter for a preconcentrator design, since complete removal of vapors from the sample stream is desirable for quantitative analysis of vapor concentrations. The capacity of a granular solid to adsorb a gas or vapor is a function of several variables including its pore size, surface area and its polarity [42]. The adsorption capacity data are usually determined by breakthrough experiments.

In a breakthrough experiment with a fixed bed adsorption system, the adsorbent located closest to the vapor inlet is the first to become saturated (i.e., to reach equilibrium). The vapor flow rapidly becomes depleted of the analyte, and therefore there is very little adsorption just downstream of the saturated zone. There is a region partially in equilibrium with the adsorbents. This region contains a concentration gradient and is called the adsorption zone. As time progresses, the adsorption zone moves deeper into the system, and eventually approaches the exit the bed (Figure 1.8 a). When the adsorption zone has moved through the adsorbent filter, the concentration of the outlet vapor will be the same as that at the inlet. This process is described by the breakthrough curve. The time-dependent concentration of the vapor at the outlet from the bed is shown in Figure 1.8.b. 

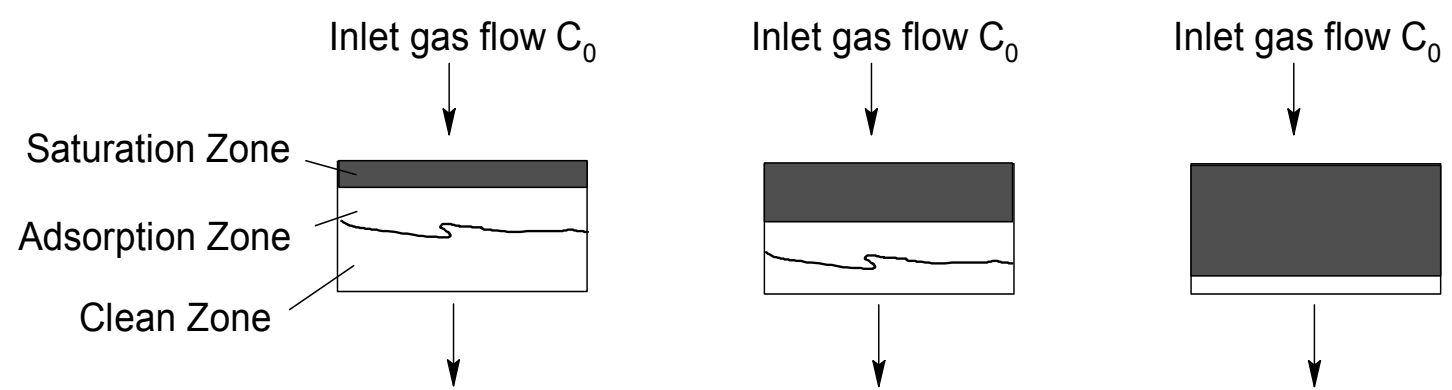

Outlet gas flow $\mathrm{C}_{1}$

Outlet gas flow $\mathrm{C}_{2}$

Outlet gas flow $\mathrm{C}_{3}$

(a)

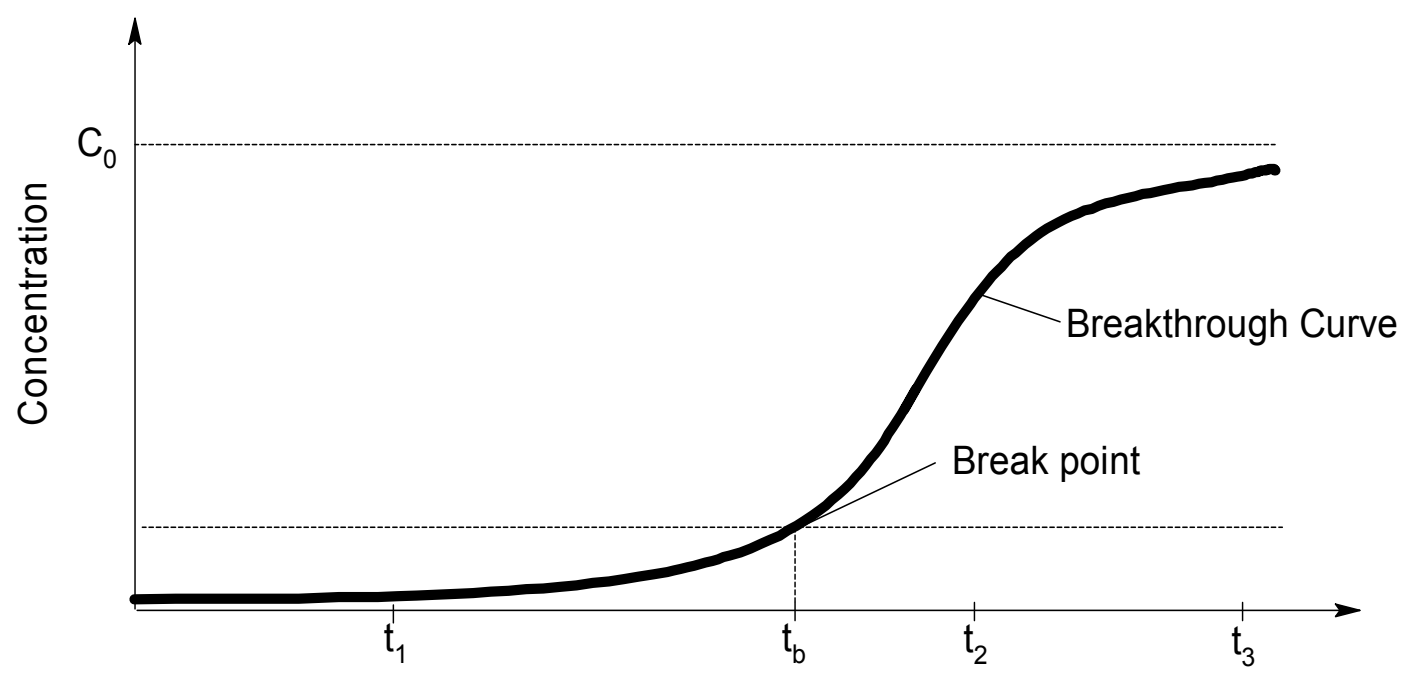

Time of Operation

(b)

Figure 1.8 (a) Illustration of vapor saturation process.

(b) A typical breakthrough curve.

The solid line shows the concentration of vapor exiting the adsorption bed. 
When a bed of fixed dimensions is exposed at a fixed flow rate to a vapor at a given concentration, the point at which saturation begins to affect performance (effluent concentration reaches a maximum permissible concentration) can be represented by the time at which vapor is first detected downstream from the adsorbent bed, which is the breakthrough time, $t_{\mathrm{b}}$. Usually, $t_{\mathrm{b}}$ is defined as the time when the concentration of the chemical in the exit stream reaches $1 \sim 10 \%$ of the inlet concentration.

The breakthrough time can be associated with the adsorption capacity by the modified Wheeler model. The modified Wheeler model relates several important design and performance parameters to the vapor adsorption capacity of a granular adsorbent bed under a continuous vapor flow [43]. It is derived from a mass balance between vapor entering, retained within, and exiting the bed, the sum of the gas adsorbed by the bed and the gas passing through the bed. Most applications of this model have been concerned with determining the service lives of air-purifying respirator cartridges packed with activated charcoal and some other adsorbent [44]. However, it has also been employed to characterize adsorbent samplers and preconcentrator systems similar to that described here [45]. The expression for modified Wheeler equation is expressed as follows [43].

$$
t_{b}=\frac{W_{e}}{C_{0} Q}\left[W-\frac{\rho_{B} Q}{K_{v}} \ln \left(\frac{C_{0}-C}{C}\right)\right]
$$




$$
\begin{array}{ll}
\text { Where } & \\
t_{\mathrm{b}} & =\text { breakthrough time }(\mathrm{min}) ; \\
\mathrm{C} & =\text { exit concentration }(\mathrm{mg} / \mathrm{mL}) ; \\
\mathrm{C}_{0} & =\text { inlet concentration }(\mathrm{mg} / \mathrm{mL}) ; \\
\mathrm{Q} & =\text { volumetric flow rate }(\mathrm{mL} / \mathrm{min}) ; \\
\mathrm{W} & =\text { weight of adsorbent }(\mathrm{g}) ; \\
\rho_{\mathrm{B}} & =\text { bulk density of packed adsorbent }(\mathrm{g} / \mathrm{mL}) ; \\
K_{v} & =\text { rate constant for adsorption }\left(\mathrm{min}^{-1}\right) ; \\
W_{e} & =\text { adsorbent capacity }(\mathrm{mg} / \mathrm{g}) .
\end{array}
$$

The values for $\mathrm{C}_{0}, W_{e}$ and $\mathrm{Q}$ are determined by the experimental test conditions, as is the temperature, which remains constant. The value of $\rho_{B}$ is determined by the physical properties of the adsorbent.

Equation 1-4 has two parameters $-W_{e}$, the adsorption capacity; and $K_{v}$, the rate constant - that can be calculated from experimental data. This equation predicts that the breakthrough time varies linearly with the weight of the adsorbent if $\mathrm{C}, \mathrm{C}_{0}, \mathrm{Q}$ and $\rho_{\mathrm{B}}$ remain constant:

$$
t_{b}=a+b W
$$


Where,

$$
\begin{aligned}
& a=-\frac{W_{e} \rho_{B}}{C_{0} K_{v}} \ln \left(\frac{C_{0}-C}{C}\right) \\
& b=\frac{W_{e}}{C_{0} Q}
\end{aligned}
$$

Experimental values of the breakthrough time $t_{\mathrm{b}}$ for the vapor and adsorbent system are obtained at a given concentration as a function of the adsorbent weight. The adsorption capacity $\left(W_{e}\right)$ and the rate constant $\left(\mathrm{K}_{\mathrm{v}}\right)$ can be found from the slope and intercept of the resulting straight line. The resulting estimate of $W_{\mathrm{e}}$ can be used for relative comparisons of adsorbents. 


\subsection{MIP Project Description}

In this project, the fundamental aspects include the following considerations. Given the targeted analytes, a monomer and a cross-linker must be chosen so that a high degree of cross-linking can be achieved (for chemical, dimensional and thermal stability). The right functional groups must be present for binding the targeted analyte, and the binding energy and kinetics of adsorption/desorption must fit the requirements of the preconcentrator. For successful imprinting, it is important that a strongly bound pre-complex is formed between the template and the monomer constituents. A strategy scheme for MIP preparation is shown in Figure 1.9. Polymerization conditions must be found to create a high density of binding sites and a degree of porosity optimized for rapid adsorption and desorption. Selectivity issues must also be addressed. For instance, it is desirable to avoid adsorption of large amounts of water to reduce interference by change in humidity, so a more hydrophobic monomer and /or cross-linker may be preferred.

As the specific structure of the cavity is determined by the fixed arrangement of the polymer chains, the optimization of the polymer structure is extremely important. The polymer should have the following properties.

a) Stiffness of the polymer structure which enables the cavities to retain their shape even after removal of the template, thus giving high selectivity.

b) Good accessibility of the vapor to as many cavities as possible in the highly crosslinked polymer.

c) Good thermal stability. The polymer must withstand the high desorption temperature in the preconcentrator application. 
We have investigated one of the most studied non-covalent, molecular imprinting systems. The system is comprised of a hydrophobic cross-linking monomer, ethylene glycol dimethacrylate, and a binding monomer, methacrylic acid and /or acrylic acid, that forms favorable hydrogen bonding with the templates (Figure 1.9). The templates, phenol or hydroquinone, are structural analogs with the similar geometry and shape with aromatic organic vapors. In order to assess MIPs for use in selective adsorption, the reference polymers (control polymers) are synthesized with no templates involved. And we intend to examine the selectivity of the imprinted polymers with small organic vapor analytes which have different molecule shapes and sizes (planar- benzene, toluene and trichloroethylene, or nonplanar- carbon tetrachloride, heptanes). 


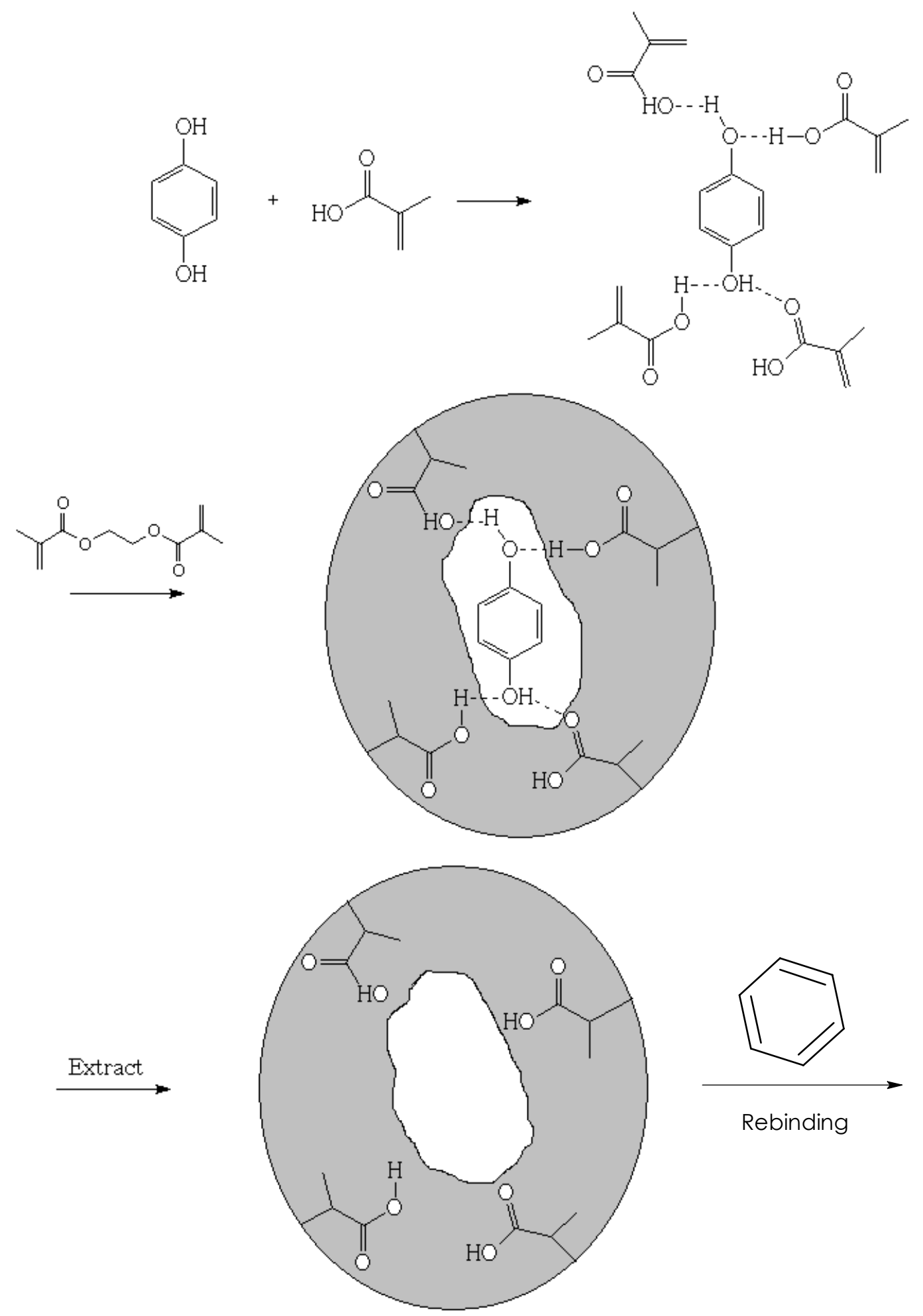

Figure 1.9 The Strategy for Creating Selective Sites within Network Polymers 


\section{Experimental Section}

\subsection{Molecular Imprinted Polymer Synthesis}

In order to assess the effect of polymerization method on the performance of imprinted polymers, a series of polymers were prepared by different polymerization protocols.

All reagents used in the experiment were of reagent grade unless otherwise described. Methacrylic acid (MAA), ethylene glycol dimethacrylate (EGDMA), acrylic acid (AA) and acrylonitrile (ACN) were from Aldrich. Azobis(isobutyronitrile) (AIBN, >98\%) was from Janssen Chemical. Hydroquinone (HQ) and phenol (P) were from Fisher. Solvents used for polymer synthesis were from commercial sources and of HPLC grade.

\subsubsection{Bulk Imprinted Polymer( BIP) Synthesis}

A bulk polymer preparation scheme was used to prepare the molecularly imprinted polymer particles with irregular shapes [46]. In a typical preparation, 2 mmol of a template such as hydroquinone (HQ) was dissolved in $5 \mathrm{~mL}$ dimethylsulfoxide, and mixed with $5 \mathrm{mmol}$ of cross-linker ethylene glycol dimethacrylate (EGDMA) and 5 mmol monomer, either acrylic acid (AA) or methacrylic acid (MAA). The solution was de-oxygenated by bubbling argon for 15 min. Then $15 \mathrm{mg}$ initiator AIBN was added. The solution was sealed in a test tube and immersed in water bath at $50{ }^{\circ} \mathrm{C}$ overnight. The resulting block polymer was ground in a mortar and pestle to form fine particles. The polymer imprinted with 
phenol (P) was synthesized using the same procedure. The control polymer (CP) was synthesized using the same procedure without any template.

\subsubsection{Imprinted Microsphere Polymer (IMP) Synthesis}

In a typical synthesis for imprinted microsphere polymer [24], $2 \mathrm{mmol}$ of a template such as hydroquinone or phenol was dissolved in $40 \mathrm{ml}$ acetonitrile with the functional monomer MAA (5 mmol) and the cross-linker EGDMA (5 mmol). The solution was de-oxygenated with argon for $15 \mathrm{~min}$, the initiator AIBN was added, and the tube was sealed and placed in a $60{ }^{\circ} \mathrm{C}$ water bath overnight. The resulting microsphere particles were then collected by centrifugation. The control polymer $(\mathrm{CP})$ was synthesized using the same procedure without the template.

\subsubsection{Molecularly Imprinted Membrane (MIM) Synthesis}

Imprinted membrane polymer, poly(acrylonitrile-co-acrylic acid) (poly(ACNco-AA)), was prepared according to the following scheme (Figure 1.10) by the phase inversion precipitate method [21].

(a) Copolymerization of acrylonitrile and acrylic acid

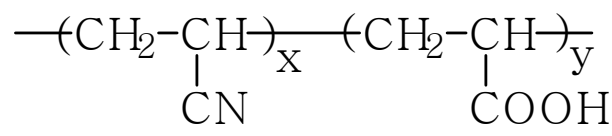


The polymerization was performed in DMSO solution at $60^{\circ} \mathrm{C}$ in a $200 \mathrm{ml}$ round bottom flask. Monomers, acrylonitrile (15 g, $0.0275 \mathrm{~mol})$ and acrylic acid (1.2 g, $0.0015 \mathrm{~mol}$ ) were well mixed with $40 \mathrm{ml}$ DMSO. The solution was saturated with argon for $15 \mathrm{~min}$ and $60 \mathrm{mg}$ AIBN was added. The polymerization was carried out at $60{ }^{\circ} \mathrm{C}$ for $4 \mathrm{~h}$. The mixture was poured into $500 \mathrm{~mL}$ methanol to precipitate the copolymer. Solidification was initiated as DMSO solution mixed with methanol. The phase separation of the polymer took place at the interface between the methanol and the polymer /DMSO solution, and a polymer membrane was obtained.

(b) Molecular imprinting process

In the imprinting process, DMSO and water were selected as the casting solvent of the copolymer and the coagulation medium, respectively. The hydroquinone template and the copolymer (poly(ACN-co-AA)) from (a) were dissolved in the DMSO cast solution in a 1:4 weight ratio. In this experiment, $2 \mathrm{~g}$ copolymer and $0.5 \mathrm{~g}$ of hydroquinone were dissolved into $20 \mathrm{ml}$ DMSO. The whole solution was well mixed at $50{ }^{\circ} \mathrm{C}$ overnight. Then the solution was cast on a QCM crystal and the crystal was immediately immersed into water at room temperature.

The membrane imprinted with phenol was obtained by the similar procedure. The control polymer was obtained without template. 


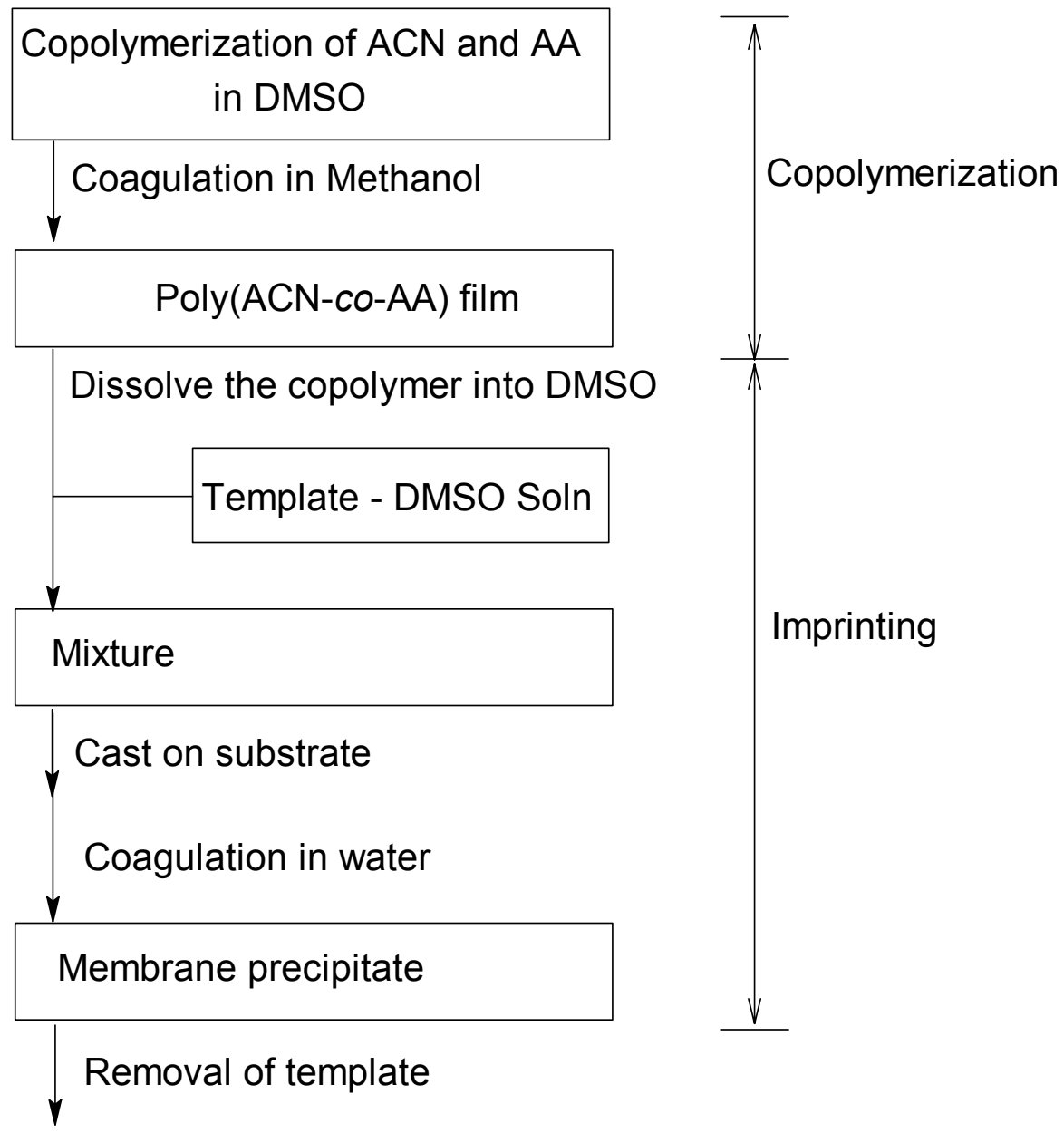

Figure 1.10 Synthesis scheme of membrane imprinted polymer (MIM) 


\subsubsection{Template extraction}

Template extraction of the polymers creates the cavities capable of specific sorption of the template and may also remove other materials from the polymer, e.g., residual monomers or oligomers and initiator fragments.

The fine particles from ground block polymer or the microspheres were placed into a round-bottom flask with a condenser and $40 \mathrm{ml}$ of methanol/acetic acid (Vol: $\mathrm{Vol}=3: 1$ ) solution was added. The slurry was stirred at $50^{\circ} \mathrm{C}$ for four hours. The extracting solvent mixture in the flask was removed by filtration and this extraction procedure was repeated with fresh solvent mixture 3 more times. The particles were isolated after filtration and dried under vacuum at $70^{\circ} \mathrm{C}$.

For Poly(ACN-co-AA) imprinted copolymer, the resulting membrane on the QCM was well washed with large amount of water and soaked in water with acetic acid to remove the solvent and the template molecule.

Template removal extraction was examined by UV-VIS absorption spectroscopy. The absorption spectrum of the standard hydroquinone solution of $10^{-4}$ $\mathrm{M}$ and the extraction solutions were taken and compared to check the template levels remaining in the extraction solutions. The UV scan was recorded between $190 \mathrm{~nm}$ and $390 \mathrm{~nm}$ and the absorption peaks near $290 \mathrm{~nm}$ were used to quantify the amount of hydroquinone template (Figure 1.11). The extraction solutions were diluted 1:200 with deionized water. The shapes of UV-VIS spectrum were similar to that of the standard solution. The spectrum of the $1^{\text {st }}$ extraction solution had a slightly different peak position around $270 \mathrm{~nm}$. We assume that the concentration of hydroquinone is 
quite high resulting in the small shift in spectrum. No absorption peak around 290 nm was observed after the $4^{\text {th }}$ washing.

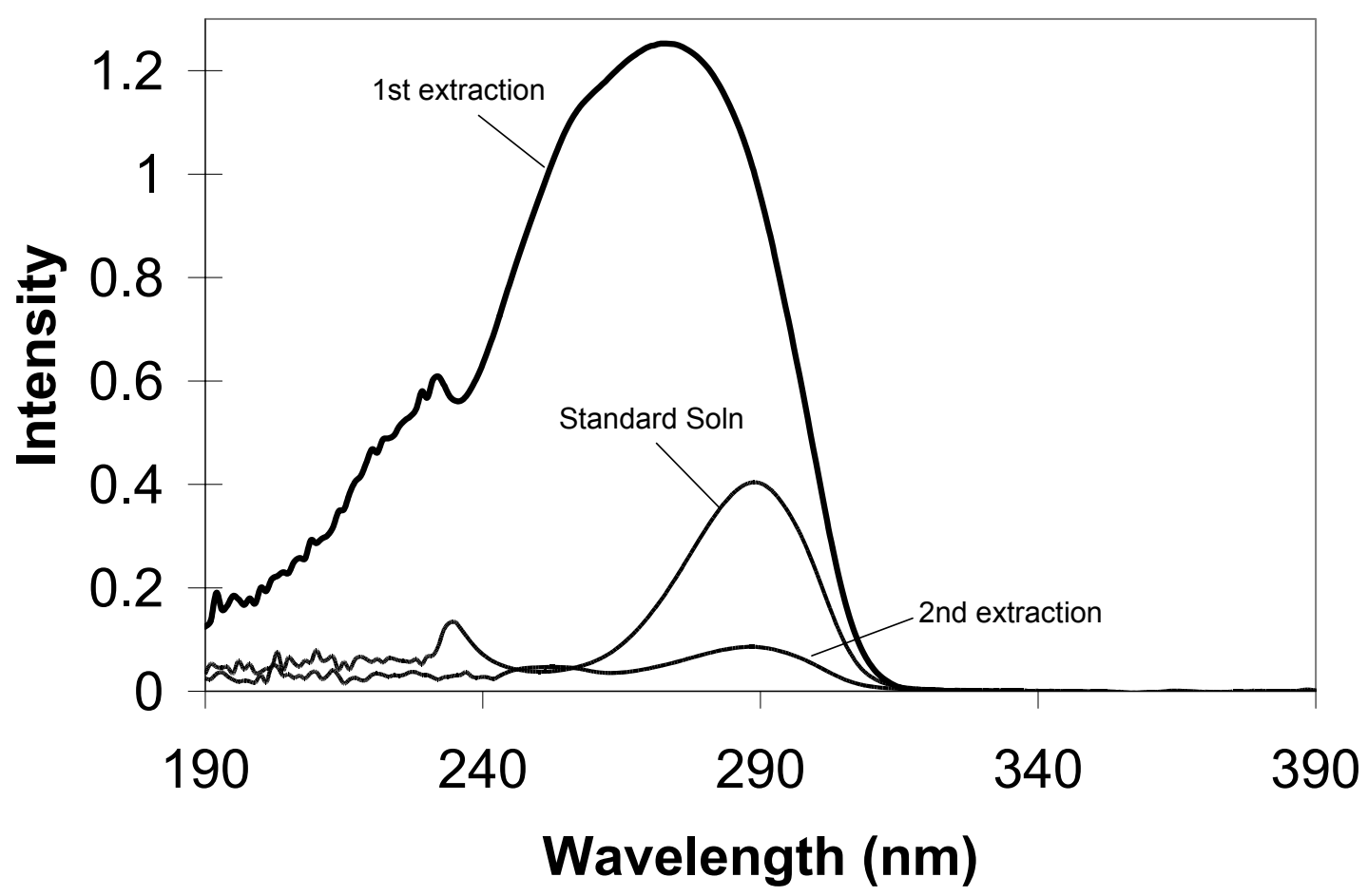

Figure 1.11 UV spectrum of template (Hydroquinone) extraction solutions. The standard solution: $10^{-4} \mathrm{M}$ hydroquinone solution 


\subsection{Vapor Generation System}

Vapors with different molecular shapes and sizes, toluene (TOL), benzene (BEZ), trichloroethylene (TCE), carbon tetrachloride $\left(\mathrm{CCl}_{4}\right)$ and heptanes $(\mathrm{HEP})$, were selected as the target vapors for the sorption/desorption tests. Toluene, benzene and trichloroethylene are planar molecules similar in size and shape to the templates hydroquinone and phenol. $\mathrm{CCl}_{4}$ represents molecules with a spherical shape and heptanes represent randomly coiled hydrocarbons.

The vapor generator used dry air obtained via a large zeolite (13X) drying column. The system purified the home compressed air using a series of filters and molecular sieves which remove particulate matter, oil and water. The purified air was then split into two separate systems. One air stream served as a reference gas and the second air stream passed through the KIN-TEK permeation source oven to generate the organic vapor stream (Figure 1.12). In this experiment, the flow rate of air stream was calibrated by a hand held flow meter (ADM1000, Humonics).

The permeation source used in this experiment consists of two types: KIN-TEK permeation refillable tubes for toluene (TOL), benzene (BEZ), carbon tetrachloride $\left(\mathrm{CCl}_{4}\right)$, trichloroethylene (TCE), and the home-made permeation tube for heptanes (HEP). The KIN-TEK refillable sources were stainless steel cylinders with two $1 / 2$ inch pipe-fitting inlet and outlet connections. The permeation tube, which was placed inside the cylinder, was filled with the liquid phase of the analyte of choice. The permeation tube was made of porous polymer membrane that separated the air stream from the organic liquid and allowed the transport of organic vapor from the liquid into the gas phase. The permeation leak rate was calibrated by weight loss of the 
tubes with respect to temperature and time. Standard emission rate correlation charts were provided for each source by the manufacture (KIN-TEK).

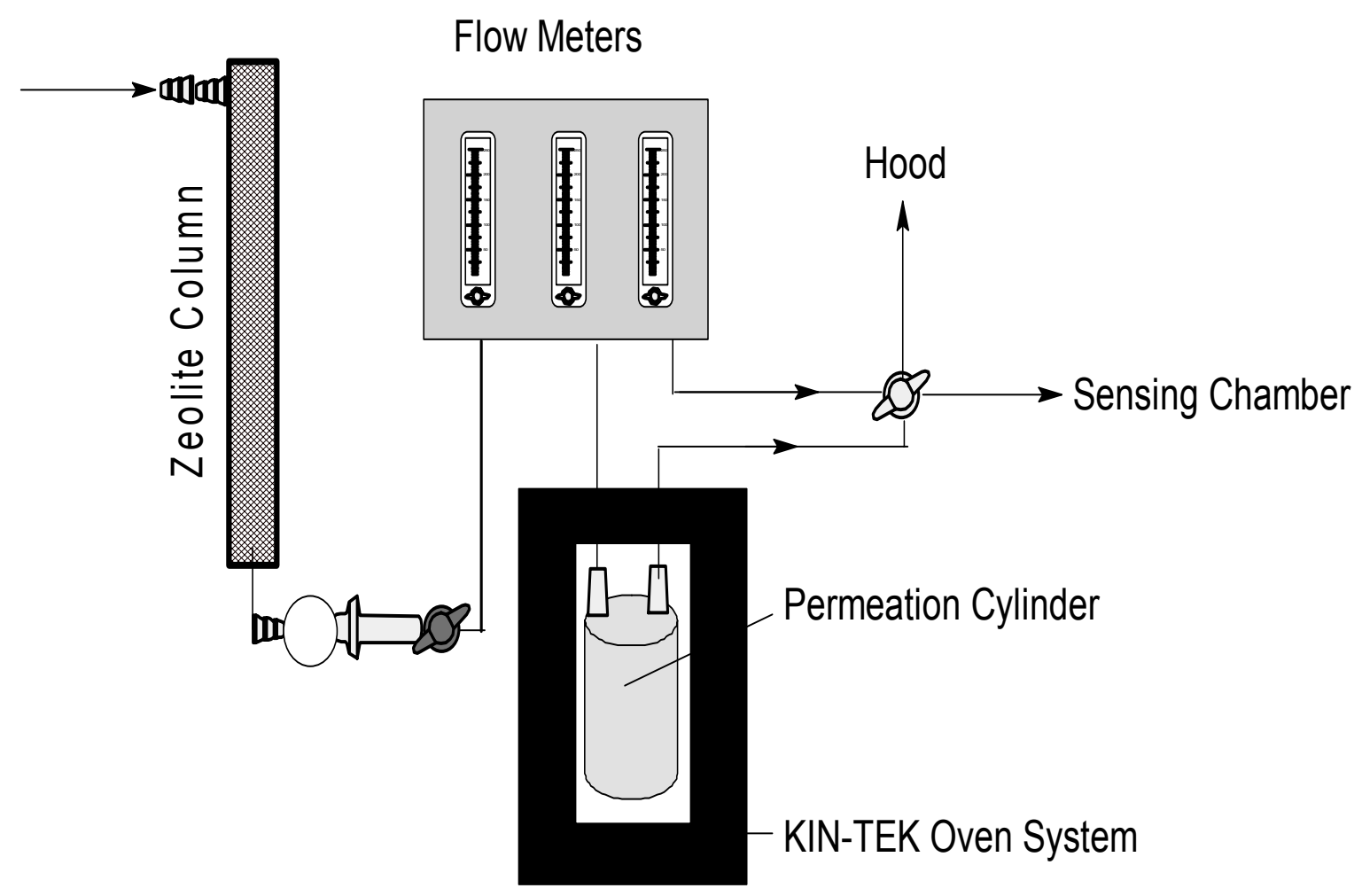

Figure 1.12 A schematic diagram of the vapor generation system 
Heptanes vapor was generated by using a homemade source design. The permeation source design consisted of a small, hollow stainless cylinder that is open in one end and covered by a folded Teflon membrane. A thin cylindrical cap secured the membrane and provided a handle. The permeation stainless cylinder was placed into a glass cylinder which can be settled in the same oven units as the refillable sources. To calibrate the source, weight change measurements were carried out over approximately two weeks. The weight loss in gram/min was converted to the emission rate $E_{R}$ in $n L / m i n$. A plot of the $\log \left(E_{R}\right)$ versus reciprocal absolute temperature provided a linear calibration curve which can be extrapolated to other temperatures and concentrations of interest (Figure 1.13).

By changing the temperature of permeation tubes and the flow rate of dilute air stream, it was possible to obtain different concentrations of the target vapor in the gas phase. The temperature was controlled by a KIN-TEK oven unit into which the permeation tubes were placed. The typical oven temperature ranged from 30 to $80^{\circ} \mathrm{C}$. The concentration of gas in the air stream is given by equation 1-8:

$$
\text { Concentration of vapor (ppmv) }=E_{R} / F_{L}
$$

Where $\mathrm{E}_{\mathrm{R}}$ is the emission rate of the permeation source in $\mathrm{nl} / \mathrm{min}$ and $\mathrm{F}_{\mathrm{L}}$ is the flow rate of air through the permeation source in $\mathrm{ml} / \mathrm{min}$. 
The KIN-TEK system was modified to supply the same flow rate of dry air stream and organic vapor stream to the QCM sensing chamber. The procedure of switching air streams was controlled manually by a valve. The permeation data of oven temperatures and corresponding vapor emission rates are given in Table 1.2.

Table 1.2 Permeation rate data of vapors

\begin{tabular}{c|c|c|c|c|c|c}
\hline Vapor & Oven Temp ( $\left.^{\circ} \mathbf{C}\right)$ & $\mathbf{3 0}$ & $\mathbf{4 0}$ & $\mathbf{5 0}$ & $\mathbf{6 0}$ & $\mathbf{8 0}$ \\
\hline Emission & Benzene $^{\mathrm{a}}$ & 7400 & 16000 & 34000 & 68000 & $\mathbf{2 4 0 0 0 0}$ \\
Rate & Toluene $^{\mathrm{a}}$ & 2800 & 6000 & 12000 & 24000 & 81000 \\
& $\mathrm{CCl}_{4}{ }^{\mathrm{n}}$ & 3000 & 7700 & 19000 & 43000 & 19000 \\
& $\mathrm{TCE}^{\mathrm{a}}$ & 31000 & 63000 & 120000 & 230000 & 730000 \\
& Heptanes $^{\mathrm{b}}$ & 8800 & 15000 & 26000 & 35000 & 74000 \\
\hline
\end{tabular}
a. Data from manufacturer;
b. Data from experiment calibration 


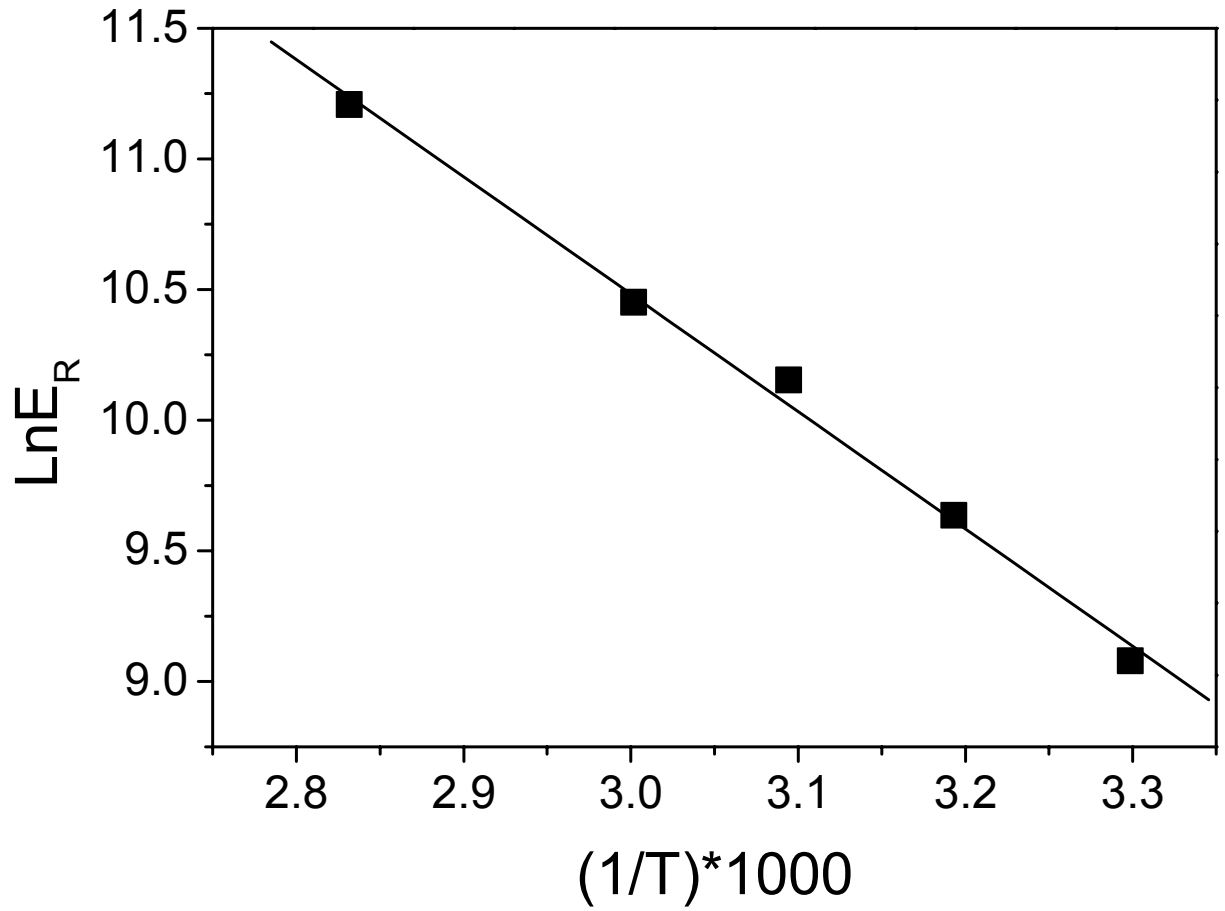

Figure 1.13 Heptanes emission calibration curve.

$E_{R}$ : emission rate ( $\left.n L / m i n\right) ; T$ : temperature in Kelvin 


\subsection{QCM Experiments}

\subsubsection{QCM Instrumental Setup}

Diagrams of the QCM apparatus are shown in Figure 1.14. The QCM flow cell consisted of an aluminum block with two similar chambers which housed the sample crystal and the reference crystal, respectively. The aluminum block was equipped with gas inlet and outlet fittings and was fixed to the base by a clamp. The rubber gasket attached to the block made a pressure seal between the block and the crystal holders. The sample chamber was connected directly to the air stream.

Piezoelectric crystals purchased from International Crystal Manufacturing Inc. were AT-cut spherical quartz crystals $(12.5 \mathrm{~mm}$ diameter) with a basic resonant frequency of $10 \mathrm{MHz}$ and two gold electrodes (6mm diameter) on opposite sides. The reference and sample crystal were connected to an oscillating circuit board through the crystal holders. The electronics of QCM, which were located beneath the sensing chamber, were designed and modified by the Finklea group [48]. The output ports of the home-made oscillator provided three outputs as sample, reference and beat frequencies. The beat frequency is the frequency difference between the sample crystal and the reference crystal. A universal frequency counter (Hewlett Packard 5384A) was employed to monitor the basic frequency of the sample crystal. The crystals were chosen so that the reference crystal had a larger base frequency than the sample crystal. In this way, an increase in the loading of the sample crystal resulted in an increase of the beat frequency. The response stability is reflected in the stability of the beat frequency which typically changed by less than $1 \mathrm{~Hz} / \mathrm{min}$. 
Data acquisition of the resultant beat frequency from the two QCM devices was accomplished with a frequency acquisition board (CIO-CTR10, Computer Board) installed in an IBM386 computer. The acquisition program presented the collected data in the form of a real time plot of beat frequency output versus time. The raw data was save as an ASCII file. The resolution of the frequency data $( \pm 1 \mathrm{~Hz})$ was set by the frequency acquisition board. 


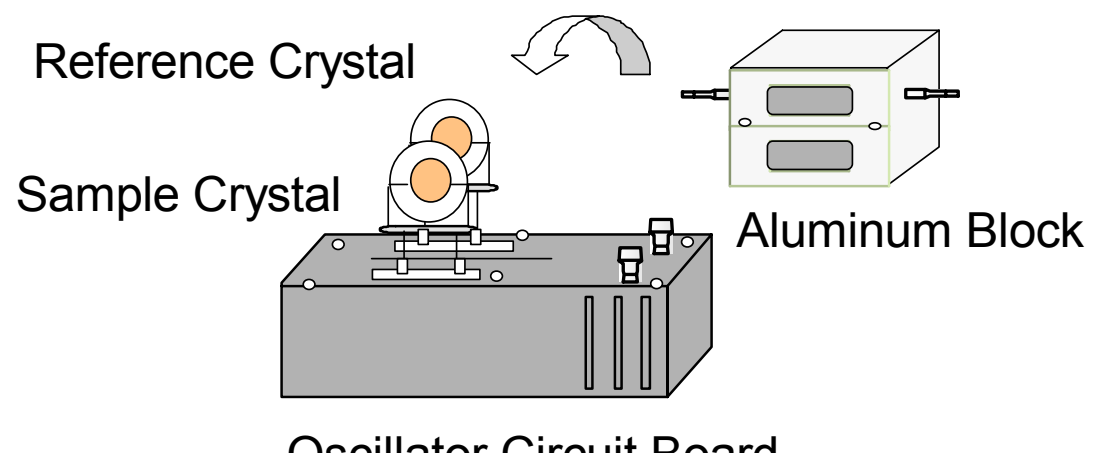

Oscillator Circuit Board

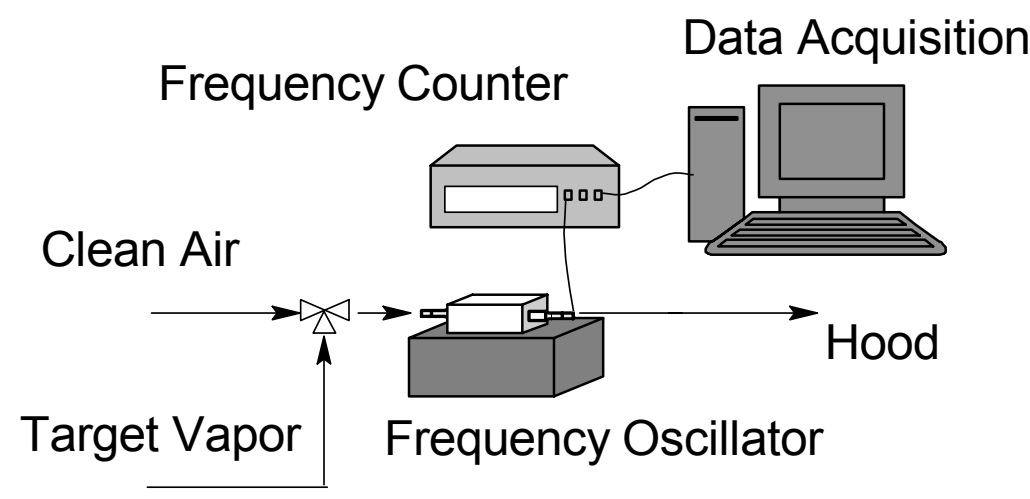

Figure 1.14 A schematic diagram of the apparatus for QCM vapor adsorption experiments. 


\subsubsection{Crystal coating}

Prior to the film deposition process, QCM crystals were cleaned using two sequential protocols: sonication and exposure to air plasma. In sonication, the crystals were immersed in a container with chloroform and sonicated by an ultrasonic Cleaner (Branson) for 10-15 minutes. After sonication, the crystals were placed in an air plasma (Harrick Scientific Corporation: Model PDC-3XG) for 10-20 minutes. The sensing crystals were considered clean when their oscillation frequencies returned to the original recorded frequencies, indicating no additional mass loading on the surface.

The ground bulk polymer was suspended in acetone and allowed to settle for $4 \mathrm{~h}$. The sedimented particles were discarded and those not sedimented were collected by centrifugation. This procedure was repeated several times. The particles which remained suspended were collected and dried to constant weight under vacuum at 60 ${ }^{\circ} \mathrm{C}$. In this way, particles with an average diameter less than $10 \mu \mathrm{m}$ were obtained [49] for use in the following experiments.

MIP particles were applied onto the QCM s using several conventional application methods, including spin coating and solvent evaporation. The spincoating apparatus was designed by Finklea group to produce thin, uniform films on crystal surface [50]. The crystal was mounted horizontally on top of a motor shaft which can rapidly accelerate to $2000-3000$ r.p.m. The deposition solution was added dropwise until the entire crystal surface is covered. At this point, the motor was switched on and the excess solvent was spun off, leaving behind a thin film of solvent 
containing the sensing material. The solvent then evaporated quickly and formed a uniform coating.

Three types of protocols were used to deposit imprinted polymers on QCM crystal surfaces. One is called direct coating, in which a trichloroethylene (TCE) suspension of polymer powder was added dropwise directly to the QCM surface before spinning. This procedure was used initially in MIP characterization experiments. In the second protocol, an inert and soluble additive such as cellulose was used as a binder to enhance the polymer particle coating. In this procedure, MIP powders were mixed with a small amount of microcrystalline cellulose (Sigma) by a fixed weight ratio of 5:1 in $20 \mathrm{ml}$ acetone. The suspension was subjected to thorough stirring for $1 \mathrm{~h}$ before coating. The other protocol is a two-step procedure, in which poly(isobutylene) (PIB) was used as a glue to enhance the particle coating. It was carried out as follows: PIB-TCE solution $(1 \mathrm{mg} / \mathrm{ml})$ was used to coat one side of the quartz crystal by the spin-coating method. Typical frequency changes associated with the deposited PIB film after one application were about $2 \mathrm{kHz}$. The polymer powder (10 mg) was suspended in $10 \mathrm{ml}$ ethanol. A few drops of the suspension was then spread on the pre-coated PIB layer. After evaporation of the ethanol at room temperature, a MIP/PIB coating was formed on the quartz crystal. The frequency became stable after $1-2 \mathrm{~h}$ period in a dry air stream.

Imprinted membrane polymers (IMP) were cast onto the crystal surface directly during the polymer imprinting process. The copolymer/template-DMSO solution was dropped onto the crystal surface till the full surface was covered. The crystal was then 
immediately immersed into water and a thin film was formed on the crystal surface. The procedure for template removal was similar to that described in section 2.14.

\subsubsection{QCM Adsorption Measurement}

During a measurement, the reference dry air stream was initially passed over the sample crystal to obtain a baseline response. Then the valve was switched to introduce the analyte vapor stream. The QCM sample crystal was exposed to the analyte stream at a constant flow rate until a stable response was obtained. After exposure, the dry air was re-introduced into the sampling chamber to re-establish the baseline.

\subsection{Vapor Uptake Measurements}

The polymer powder was heated to $\sim 80{ }^{\circ} \mathrm{C}$ for $1-2 \mathrm{~h}$ under vacuum to remove all adsorbed material. About $0.5 \mathrm{~g}$ of the fresh polymer powder was weighed out with high precision and loaded into a pre-weighed small sample vial $(4 \mathrm{~mL})$. The vial was then placed into a mid-size empty desiccator. A small amount of organic solvent was injected into the desiccator to form an air environment saturated with the organic vapor. The desiccator was sealed and the vapor exposure was maintained for 24 hours. The mass of the powder was measured after the treatment. After weighing, the polymer powder was reheated under vacuum as described previously, and then exposed a second time to air saturated with organic vapor. Average mass uptakes of the two adsorption steps were converted to percent mass increase. 


\subsection{Breakthrough Tests}

\subsubsection{General Setup}

The adsorbent tubes were custom-made of glass tubing, about $7 \mathrm{~cm}$ long and 3 mm ID as shown in Figure 1.15. The tube ends were closed with rubber caps. The tubes were packed with different amount of adsorbents (Tenax TA, GR or homemade molecularly imprinted polymers) in the range of 5 to $30 \mathrm{mg}$. The adsorbents were held in place with glass fiber plugs (Fisher). Various known concentrations of vapors were prepared as described previously, and passed through the tubes at a stable flow rate. Downstream of the sampling tube was a flame ionization detector (FID), which was used to monitor the vapor concentration passing through the adsorbent tubes. The current signal from the FID was recorded by a Tektronix oscilloscope.

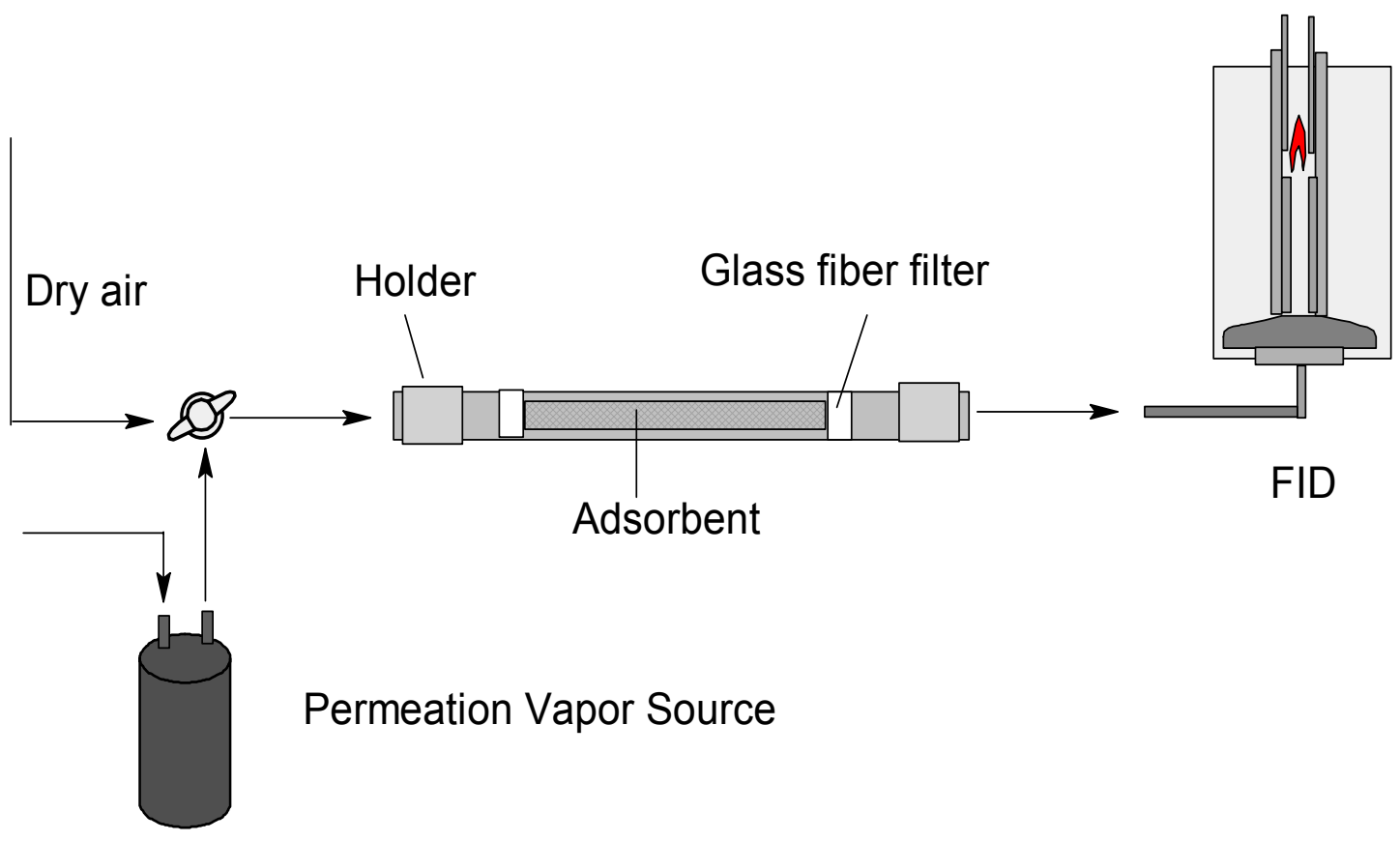

Figure 1.15 The set-up for Breakthrough experiment 


\subsubsection{Flame Ionization Detector (FID)}

The Aerograph FID used in this experiment is designed for the Series 3700 Gas Chromatograph (Varian). The detector flame was generated from a mixture of $200 \mathrm{~mL} / \mathrm{min}$ of air and $30 \mathrm{~mL} / \mathrm{min}$ of hydrogen. The FID temperature was maintained at $150{ }^{\circ} \mathrm{C}$. Since the FID signal is sensitive to the flow rate, flow rates of gas streams were kept constant during the experiment. For the target analytes (Toluene, Benzene, Heptanes) in this experiment, the FID response indicated good linearity over a wide concentration range (Figure 1.16).

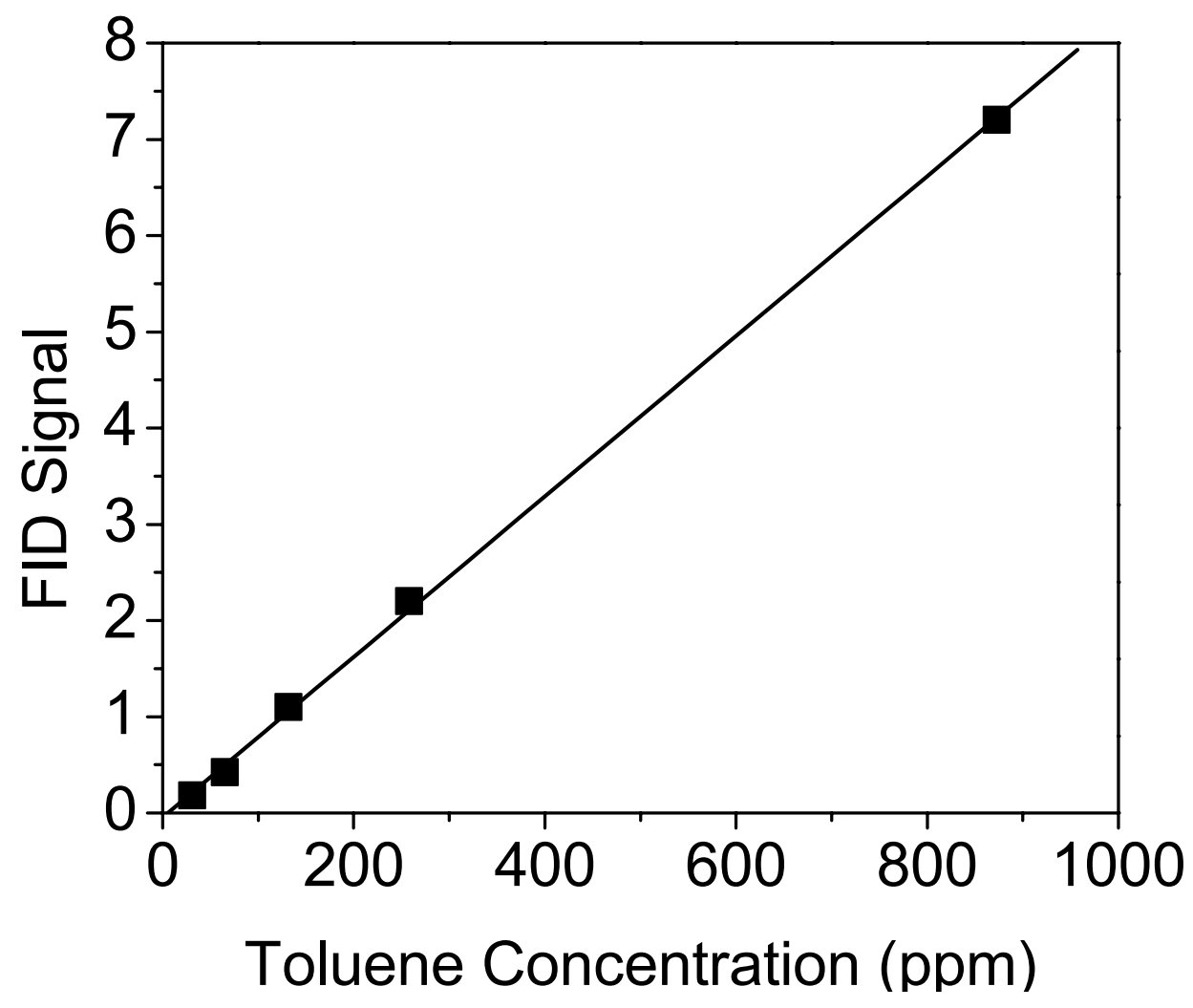

Figure 1.16 The calibration curve of toluene vapor with the FID detector 


\subsubsection{Breakthrough Experiment}

The adsorbents were conditioned at $100{ }^{\circ} \mathrm{C}$ for 2 hours before testing. After packing, dry air was pulled through the tube for $2 \mathrm{~h}$ before tests. The FID signal corresponding to inlet vapor concentration was first determined by introducing the desired concentration of the vapor stream through an empty tube. The empty tube was removed and the packed tube was placed into the holder. The vapor was then pulled through the adsorbent and the FID response was recorded. After the penetration of the cartridge reached approximately $20 \%$ (i.e., the FID signal reached $20 \%$ of the original signal measured previously), the vapor stream was switched off and the cartridge was removed from the holder and dismantled. The breakthrough plot was saved for analysis.

The tests were repeated for the cartridges packed with different amounts of adsorbents. Breakthrough times for the selected $10 \%$ breakthrough fraction were obtained from breakthrough plots. Linear regression analysis of breakthrough time vs. adsorbent mass yielded estimates of adsorbent capacity $W_{e}$ and rate constant $\mathrm{K}_{\mathrm{v}}$ via Eq (1-7). 


\section{Results and Discussion}

\subsection{Polymer preparation}

Suitable conditions for the synthesis of molecularly imprinted polymers are examined by varying factors likely to affect the selectivity of the materials toward the given template. For this purpose, an initial screening of the monomers, cross-linkers and porogens is performed. This procedure allows the batch size to be reduced and a large number of samples to be synthesized and assessed in a short time.

A series of polymers are prepared to get information on how the composition of polymerization mixtures influences the properties of the resulting polymers. Two major types of polymers are prepared, highly cross-linked copolymers based on ethylene glycol dimethacrylate (EGDMA) and a membrane type of copolymer based on acrylonitrile $(\mathrm{ACN})$. The EGDMA cross-linking monomer represents the main component of the monomer mixture. EGDMA has been successfully used in various protocols [15]. The relatively low viscosity of EGDMA makes it advantageous in homogenizing the monomer mixture prior to polymerization. Thus, this cross-linker is well documented as an ideal cross-linker for non-covalent imprinting [51].

Mono-functional monomers containing acidic groups, acrylic acid (AA) and methacrylic acid (MAA) are used mostly in this experiment in an attempt to form hydrogen-bonding with the templates, hydroquinone (HQ) and phenol (P). 
Test polymerizations are carried out in a range of solvents commonly used in molecular imprinting: toluene, chloroform, acetonitrile, acetone and dimethylsulfoxide (DMSO). In this study, DMSO and acetonitrile are finally selected as the solvent porogens because they have good capability to dissolve the templates ( hydroquinone and phenol) in a sufficiently high concentration. The polymers formed in acetone and chloroform are fragile and do not show good thermal stabilities in the thermal desorption studies (Chapter II).

The effect of the polymerization temperature is quite striking. It is thought that higher temperature might result weaker monomer/template complexes since poor selectivity for the polymers synthesized under higher temperatures was observed as reported [52]. Based on our experience, the most effective polymers seem to be those polymerized at $40-60{ }^{\circ} \mathrm{C}$.

Polymerization Block imprinted polymer (BIP) are performed at $50{ }^{\circ} \mathrm{C}$ for 24 hours. The reactions are rapid; the change from a solution to a gel form is observed about 10- 20 minutes after the addition of initiator AIBN. During the polymerization, the transparency of the starting polymerization mixture does not change. A small volume contraction is observed and the hard, block-shaped polymers are obtained.

The polymerization of imprinted microsphere polymers (IMP) is achieved with a much more dilute monomer solution (approximately 5 vol\% total monomer loading relative to the porogen solvent). The polymerizations are slower. The first sign of polymerization occurs after $1 \sim 2 \mathrm{~h}$. The initial clear solution becomes cloudy during the polymerization. An attractive feature of the microspheres is that the polymer suspension is easy to prepare and disperse using a pipette. A fast separation 
of the microspheres with small and uniform sizes can be readily obtained by centrifugation.

Molecularly imprinted membranes (MIM) are synthesized by a different protocol, the phase inversion precipitation method. The solidification is initiated by the transition from the liquid state of the cast solution to two liquid phases having cast solvent (DMSO) and coagulate solution (water). The polymer solidification takes place in the presence of template, and hence the resultant polymer film contains approximate size of the template in the flat polymer membrane.

\subsection{QCM Results for MIPs}

Measurement of vapor adsorption in a coating on a QCM is an extremely useful quantitative method to directly detect molecular recognition. In evaluating the MIP coatings on QCM, measurements are focused on the following determinations: 1) real-time response patterns of vapor adsorption/desorption; 2) partition coefficient ; 3) reproducibility and 4) life time stability. The effects of coating methods and coating thickness are also examined. Without a standard coating procedure capable of assuring reproducibility, the observations obtained from single experiments only have limited value. 


\subsubsection{Formation of MIP Coatings on QCM}

\subsubsection{Molecularly imprinted membrane (MIM) coatings on QCM}

Molecularly imprinted membranes (MIMs) based on acrylonitrile form stable and adherent coatings on QCM crystal surfaces with direct casting methods. The solution of copolymer and template mixture is cast directly onto the crystal surface, and the crystal is immersed into water to precipitate the film. After the coagulation, the coated crystal is soaked win a large amount of acetic acid/methanol to remove the template molecule. After the coated film is dried, the change in the frequency of the QCM provides a measure of the amount of coating applied. A set of data listed in Table 1.2 was obtained to study the reproducibility of the MIMs coating preparations. Five crystals with the same amount $(5 \mu \mathrm{L})$ of coating material of MIM (HQ) ${ }^{*}$ are tested. For comparison, all of the measurements were conducted using the same casting solutions. The average of frequency change $\Delta f_{s}$ is $27.3 \mathrm{kHz}$ with a relative standard deviation (RSD) of $2.1 \%$. When exposed to $300 \mathrm{ppm}$ toluene vapor, the average of associated frequency shifts $\left(\Delta f_{v}\right)$ is $64 \mathrm{~Hz}$, and the average of calculated partition coefficients (K) is 2090 with a RSD of $0.76 \%$. The RSD values suggest good reproducibility of both the imprinting and coating processes.

\footnotetext{
* An abbreviation of MIM polymers imprinted with hydroquinone (HQ)
} 
Table 1.3 Frequency changes of the quartz crystal coated with MIM (HQ) films and the associated partition coefficient $(K)$ data. Test vapor: 300 ppm Toluene.

\begin{tabular}{c|cccccc}
\hline Crystal & $\mathbf{1}$ & $\mathbf{2}$ & $\mathbf{3}$ & $\mathbf{4}$ & $\mathbf{5}$ & RSD (\%) \\
\hline$\Delta \boldsymbol{f}_{\boldsymbol{s}}(\mathrm{k} \mathrm{Hz})$ & 27.8 & 26.9 & 26.5 & 27.2 & 28.1 & 2.1 \\
$\Delta \boldsymbol{f}_{v}(\mathrm{~Hz})$ & 65 & 64 & 64 & 65 & 64 & 0.76 \\
$K$ & 2070 & 2110 & 2140 & 2120 & 2020 & 2.0 \\
\hline
\end{tabular}

\subsubsection{EGDMA MIP coatings on QCM}

Molecularly imprinted polymers based on EGDMA (BIPs and IMPs) are rigid glassy materials and are insoluble in all solvents. In the initial efforts in the project, the polymer powder suspension is applied on the QCM surface by spincoating to form a thin coating. After solvent evaporation, the polymer powders do not adhere well to QCM surfaces as shown by small frequency changes $(<1 \mathrm{kHz})$ after deposition of a visibly heavy coating of MIP powders. Although the preliminary results indicate a selective pattern of adsorption with a freshly prepared QCM (Section 3.3), the coated QCM only shows short term stability (i.e., the coated QCM loses at least $50 \%$ of its original response after a week).

Because of the problems related to the formation of a particle coating on QCM via physical bonding, using additives as a "glue" component seems to be a more practical alternative. In previous reports, poly (vinyl chloride) (PVC) or 
cellulose were used as binders to enhance the adhesion of insoluble particles such as graphite particles or MIP powders to QCM surfaces [53]. Cellulose itself contributes very little to the organic vapor adsorption. The response of QCM coated with $5 \mathrm{kHz}$ cellulose only causes $1 \sim 2 \mathrm{~Hz}$ frequency changes when exposed to most tested organic analytes at a $300 \mathrm{ppm}$ concentration level. Such a small response is negligible in comparison to the QCM with polymer coatings. Cellulose is selected as a candidate binding material to enhance the adhesion between the MIPs and the QCM crystal surface. The microcrystalline cellulose is dissolved in acetone, mixed with MIP powders, and spread onto the QCM surface. Polymer particles are left with the cellulose additive on the QCM surface after solvent evaporation. Since we are unable to determine the contribution of the cellulose binder to the frequency change of the coating step in each different coating, we base the selectivity assessment in these trials generally upon comparison of vapor-induced frequency changes obtained from a single QCM with the same coating.

A key feature of the second protocol is sequential deposition of the "glue" layer and the MIP particles. Poly (isobutylene) (PIB) is a "rubbery" polymer which has been used as a traditional piezoelectric sensor coating for non-polar or weakly polar organic analytes because of its rapid and reversible equilibration with organic vapors [54]. PIB forms adherent, smooth and "sticky" coatings on the QCM surface and greatly improves the adhesion of the MIP powders to the QCM. In addition, the response of PIB coatings to the organic vapors is well established (Figure 1.17 and Section 3.2.2 ). Figure 1.18 shows the response of a $14 \mathrm{kHz}$ PIB coating upon exposure to increasing levels of toluene vapor. The PIB film exhibits fast adsorption 
and desorption behaviors for different toluene concentrations. The fast rise and decay times indicate the rapid diffusion of analyte molecules through the polymer matrix. In addition, the PIB film shows reversible responses back to the original baseline at different concentration levels. Based on the above results and our experience, a successful coating procedure is finalized by the sequential deposition of the PIB layer and MIP particles by spin-coating and vapor evaporation methods, respectively. The net frequency changes associated with MIP sorption are obtained by subtracting the predicted frequency changes for the PIB coating from the observed frequency change. This procedure is based on the assumption that physical adsorption properties of PIB are not affected by the additional MIP layer. Unless otherwise specified, frequency changes and partition coefficients for MIP coatings in this experiment are corrected for the contribution of the PIB layer to the total frequency change. 


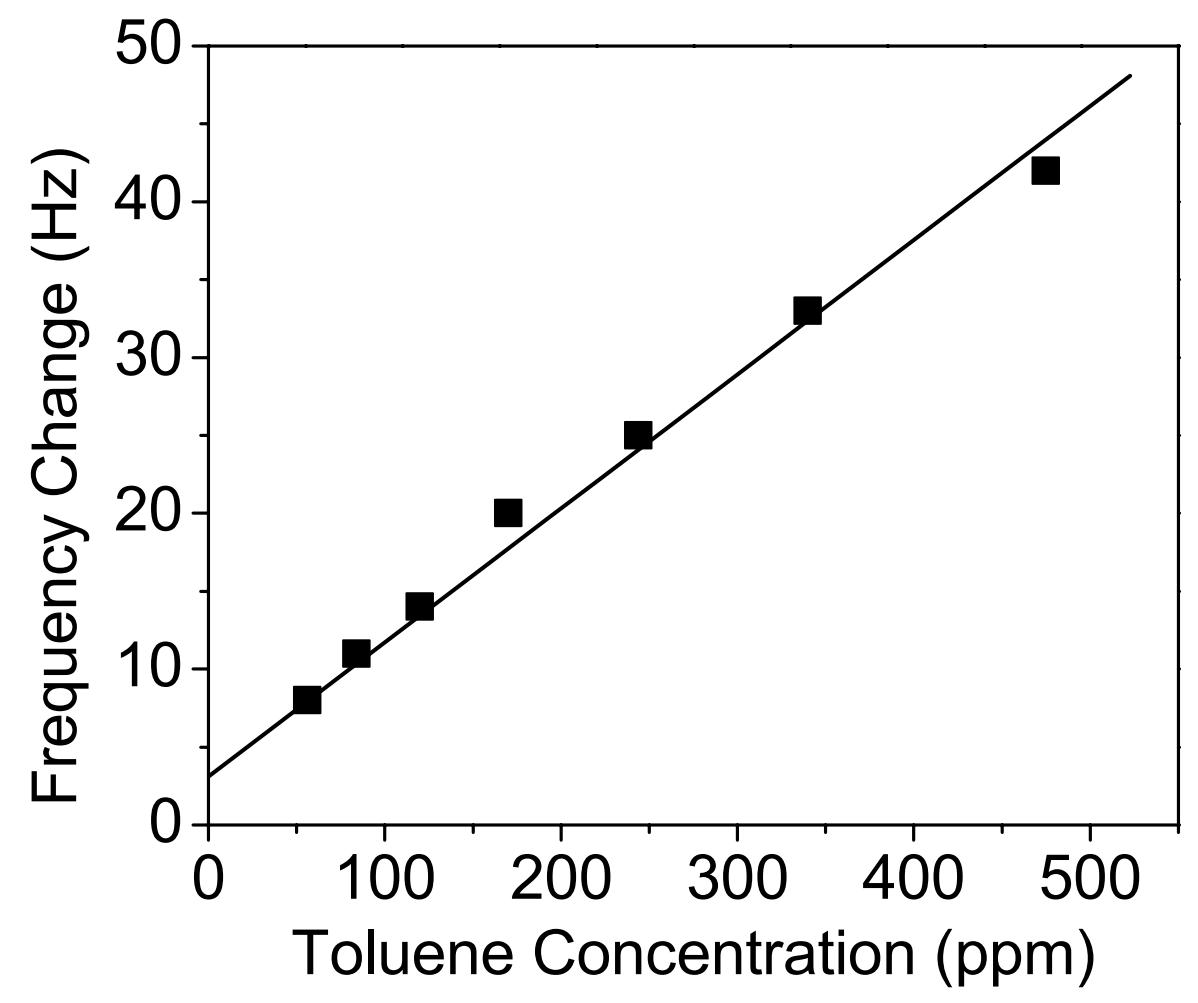

Figure 1.17 The calibration curve for a QCM coated with PIB layer to toluene vapor. PIB layer coating: $14 \mathrm{kHz}$; Partition coefficient $K=2,000 ; R^{2}=0.9845$. RSD of $K$ values based on 3 trials is $1.6 \%$. 


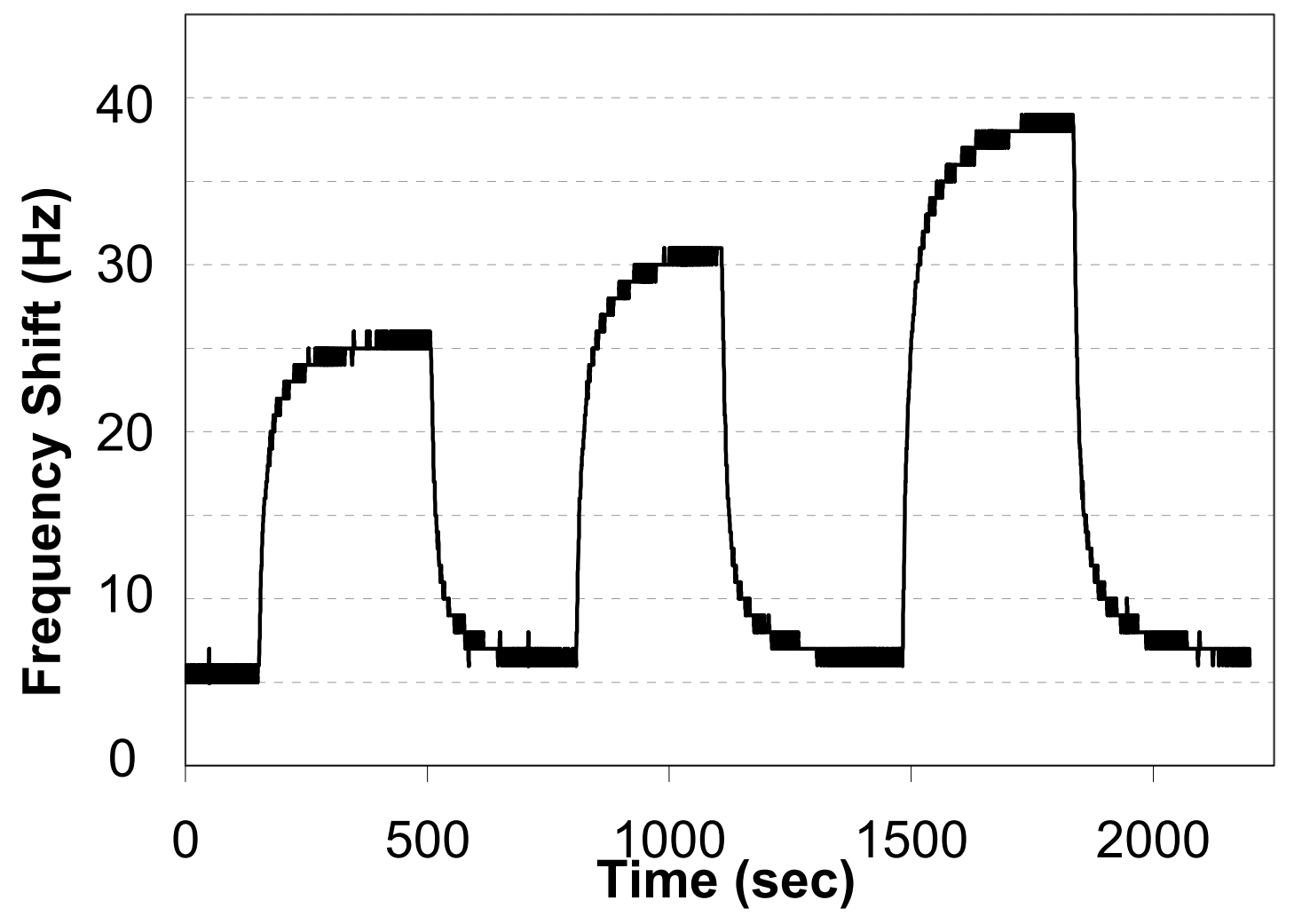

Figure 1.18 A QCM coated with PIB layer responses to 170 ppm, 240 ppm and 340 ppm toluene vapor, respectively. PIB layer coating: $14 \mathrm{kHz}$ 


\subsubsection{Calibration Curves}

Calibration curves are based on the steady-state frequency change of the QCM after the introduction of the organic vapor in the air stream. Some examples of calibration curves are shown in this section. As noted in Section 3.2.1.2, the calibration curves for the methacrylic imprinted polymer (BIPs and IMPs) are based on frequency changes corrected for adsorption by the PIB binding layer. The calibration curves for a $3 \mathrm{kHz}$ coating of Block Imprinted Polymer (BIP) imprinted with hydroquinone (HQ) are shown in Figure 1.19. The coated QCM sensor was studied with vapors of TOL, BEZ, HEP and $\mathrm{CCl}_{4}$ (since the permeation rate for TCE vapor source is too high to obtain a concentration level lower than $100 \mathrm{ppm}$, TCE vapor is excluded in this study). In each case, the frequency shift of both BIP and PIB coatings vary linearly with the vapor concentration for vapor concentrations of $30-400 \mathrm{ppm}$. Correlation coefficient $\left(\mathrm{R}^{2}\right)$ range from 0.953 to 0.999 . The linearity of the plots supports the application of Equation 1-3 :

$$
K=\Delta f_{v} \rho / \Delta f_{s} C_{v}
$$

to obtain the apparent partition coefficient K. Apparent partition coefficients based on the linear regression slopes are given in the figure captions. It is immediately evident that the BIP polymer exhibits substantially higher partition coefficients than PIB for toluene and benzene, while partition coefficients are only slightly increased 
for the nonplanar carbon tetrachloride and heptanes. This is the initial evidence for a successful molecular imprinting effect on adsorption from the gas phase.

Calibration curves for IMP polymers are shown in Figure 1.20. The QCM was coated with $3 \mathrm{kHz}$ IMP polymer powders plus $2 \mathrm{kHz}$ PIB glue layer. Excellent linear relationships between $\Delta f$ and vapor concentrations are obtained. The calculated partition coefficients for TOL and BEZ based on linear regression slopes are smaller when compared with partition coefficients for BIP polymers. However, increased sensitivity toward TOL is still observed in the IMP film. The $\mathrm{K}$ value is about 4 times higher than the K values of PIB layer.

Figure 1.21 shows the calibration curves for molecularly imprinted membranes (MIM) imprinted with hydroquinone (HQ) for TOL, BEZ and $\mathrm{CCl}_{4}$. The response of MIM film to the organic vapors is relatively small. A much thicker coating must be deposited onto the QCM to achieve a comparable $\Delta f$. The measured $\mathrm{K}$ values are roughly 1 order of magnitude smaller than the $\mathrm{K}$ values for the BIP coating. The sensitivity of the film is not much better than that of PIB.

The large volume of response data needed to evaluate the MIPs and the linear calibration curves in figures lead us to use a single concentration point for comparison of partition coefficients. In most of the experiments, a concentration level of $300 \mathrm{ppm}$ for different organic vapors is adopted for selectivity comparison. This concentration point is located in the linear region of most calibration curves. It is achievable for most vapor sources operated under mild permeation temperatures. The linear regression fits of the calibration curves yield positive intercepts. The cause for the positive intercepts is unclear. The non-zero intercepts result in an error in the 
apparent partition coefficient which is calculated at a single vapor concentration. For BIP (HQ) polymer at $300 \mathrm{ppm}$ vapor concentration, the single point partition coefficients are higher than the partition coefficients from the slope of the calibration curve by $13 \%, 15 \%$, and $20 \%$ for toluene, benzene and $\mathrm{CCl}_{4}$, respectively. This error is particularly high for the more weakly adsorbed vapors, generally when $\Delta f$ goes below $10 \mathrm{~Hz}$. In this case, the error of $\mathrm{K}$ is in excess of $100 \%$ for heptanes. Nevertheless, the single point calibration results are used for rapid comparison of many different coatings. 


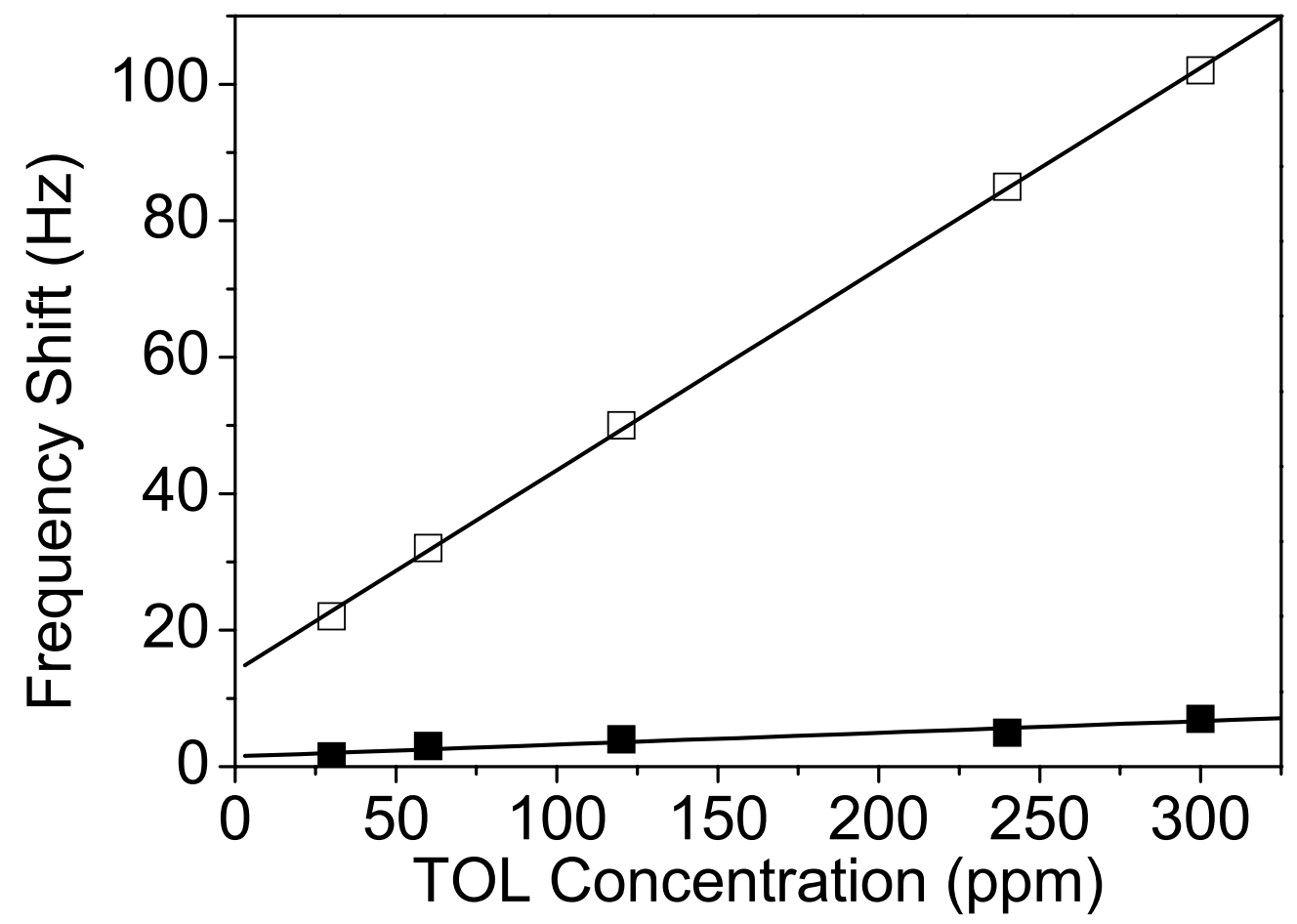

Figure 1.19 Block Imprinted Polymer (BIP) Calibration curve.

(a) Toluene vapor

Solid square: PIB coating: $2 \mathrm{kHz}, \mathrm{y}=1.52+0.0172 \mathrm{x}, \mathrm{R}^{2}=0.931, \mathrm{~K}=2,300$.

Hollow square: Polymer layer coating: 2 kHz PIB coating plus 3 kHz block methacrylic polymer (imprinted with hydroquinone); frequency changes are corrected for the contribution of the PIB layer to the total frequency change. $y=14.0$

$$
+0.295 x ; R^{2}=0.999, K=26,000 \text {. }
$$

RSD of frequency shifts based on 3 trials is $1.4 \%$ for BIP and $3.2 \%$ for PIB. 


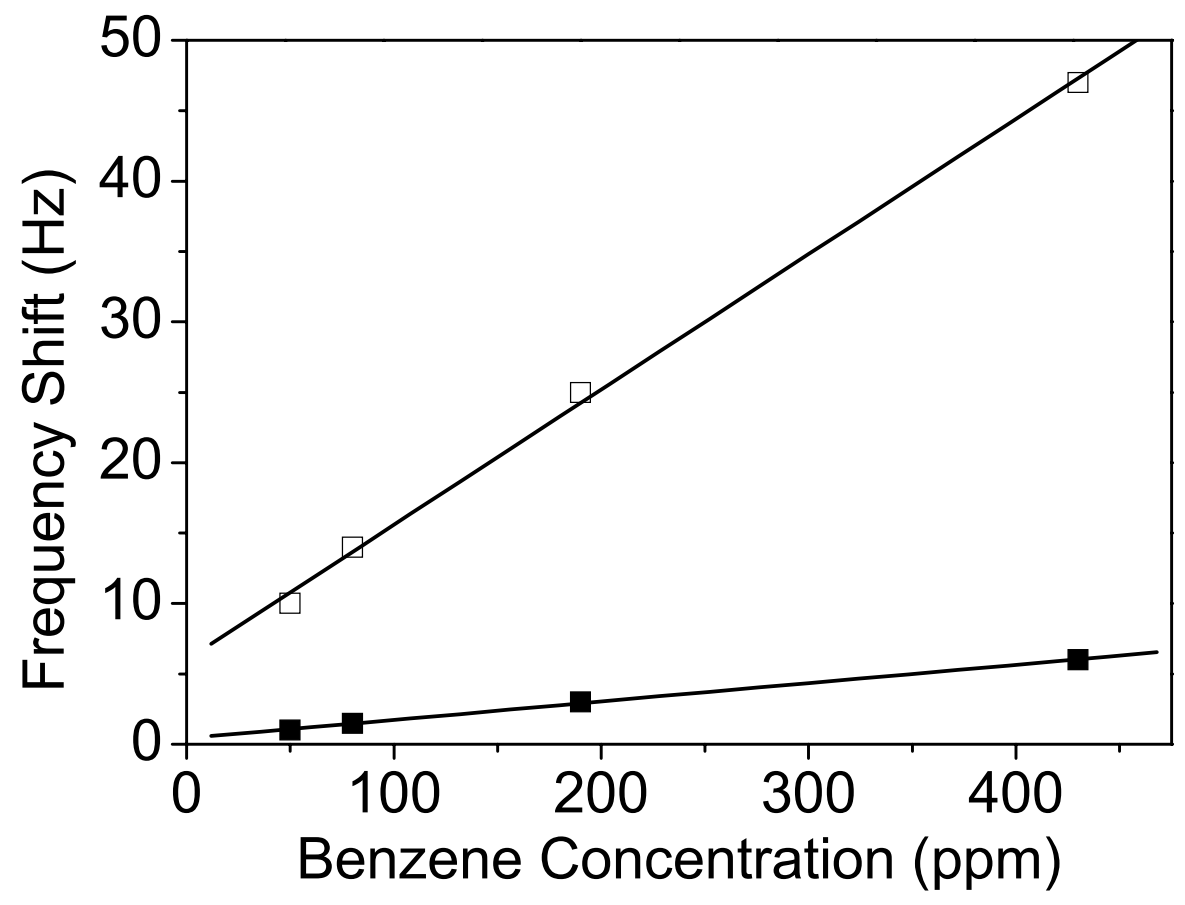

Figure 1.19 (b). Benzene vapor

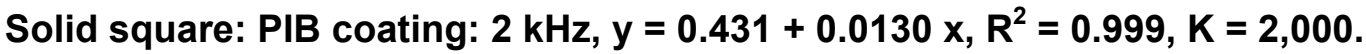

Hollow square: Polymer coating: as in $1(a), y=5.98+0.0961 x, R^{2}=0.998, K=$ 10,000 .

RSD of frequency shifts based on 3 trials is $2.4 \%$ for BIP and $3.6 \%$ for PIB 


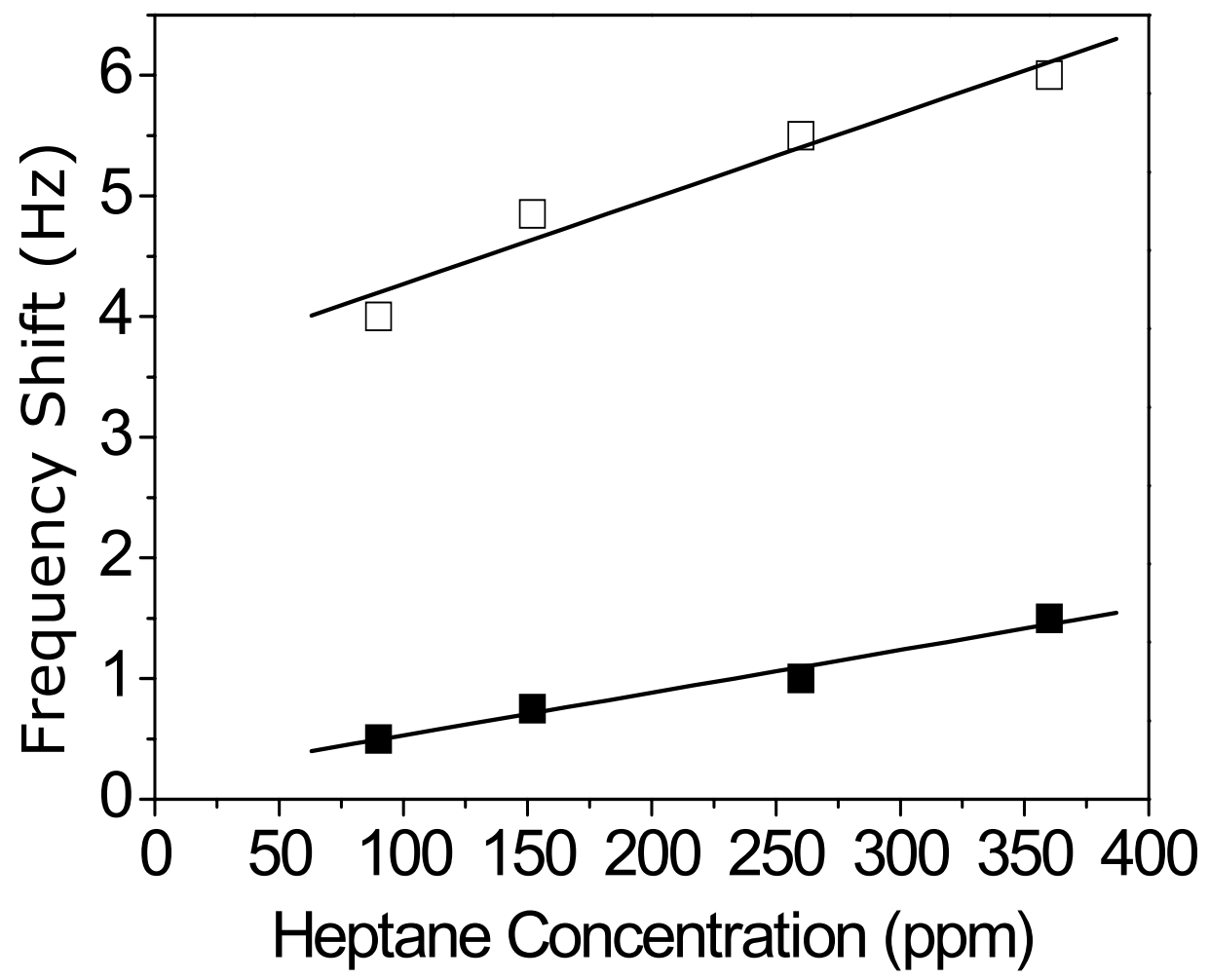

Figure 1.19 (c). Heptanes vapor

Solid square: PIB coating: $2 \mathrm{kHz}, \mathrm{y}=0.175+0.00354 x, \mathrm{R}^{2}=0.976 ; \mathrm{K}=430$.

Hollow square: Polymer coating as in $1(a), y=3.56+0.00708 x, R^{2}=0.953, K=$ 580. RSD of frequency shifts based on 3 trials is $3.4 \%$ for BIP and $4.8 \%$ for PIB 


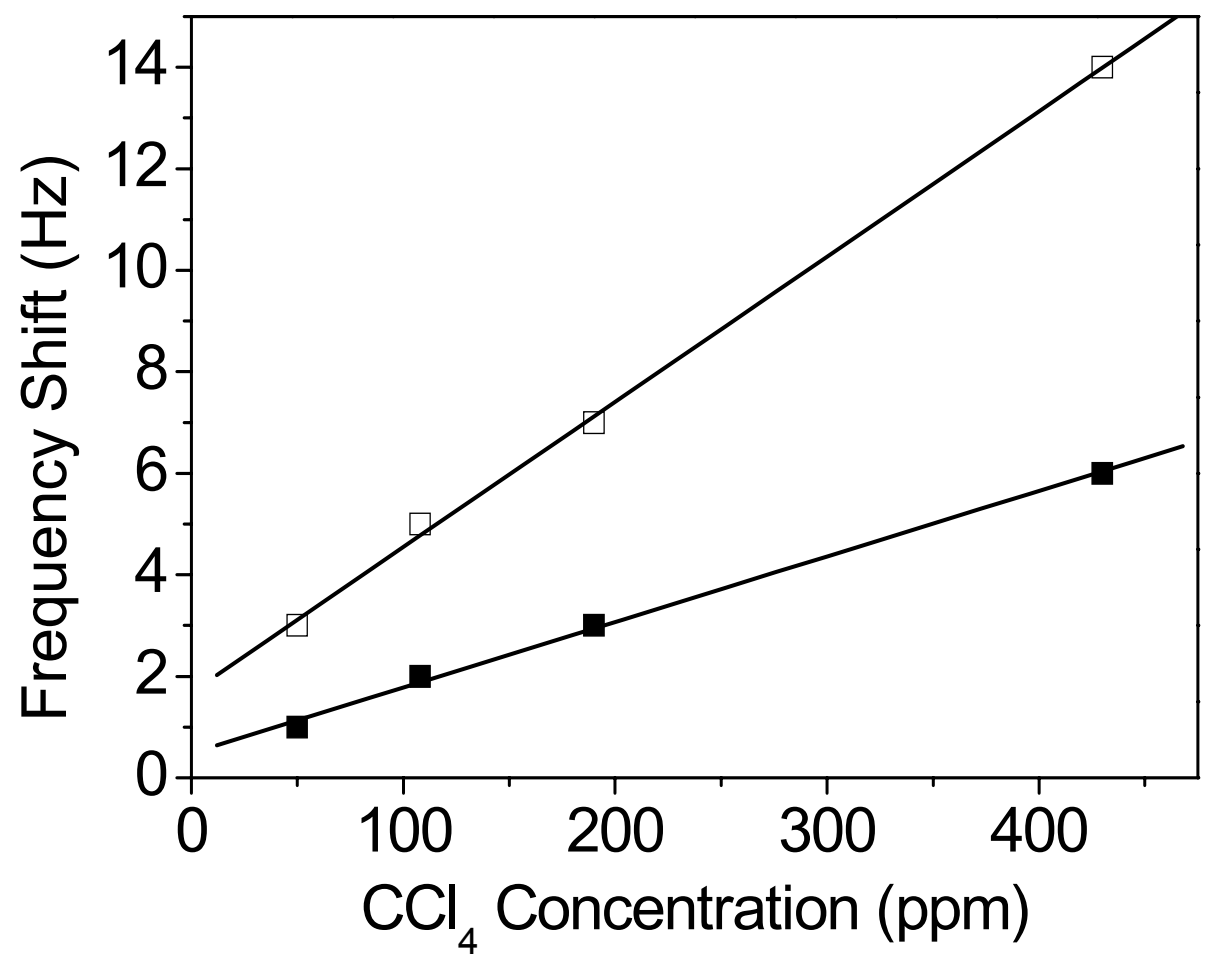

Figure 1.19 (d). Carbon tetrachloride vapor.

Solid square: $\mathrm{PIB}$ coating: $2 \mathrm{kHz}, \mathrm{y}=0.490+0.0129 \mathrm{x}, \mathrm{R}^{2}=0.997, \mathrm{~K}=1,000$.

Hollow square: Polymer layer coating: as in $1(a), y=1.68+0.0286 x, R^{2}=0.999$,

$$
K=1,500 \text {. }
$$

RSD of frequency shifts based on 3 trials is $2.8 \%$ for BIP and $4.1 \%$ for PIB 


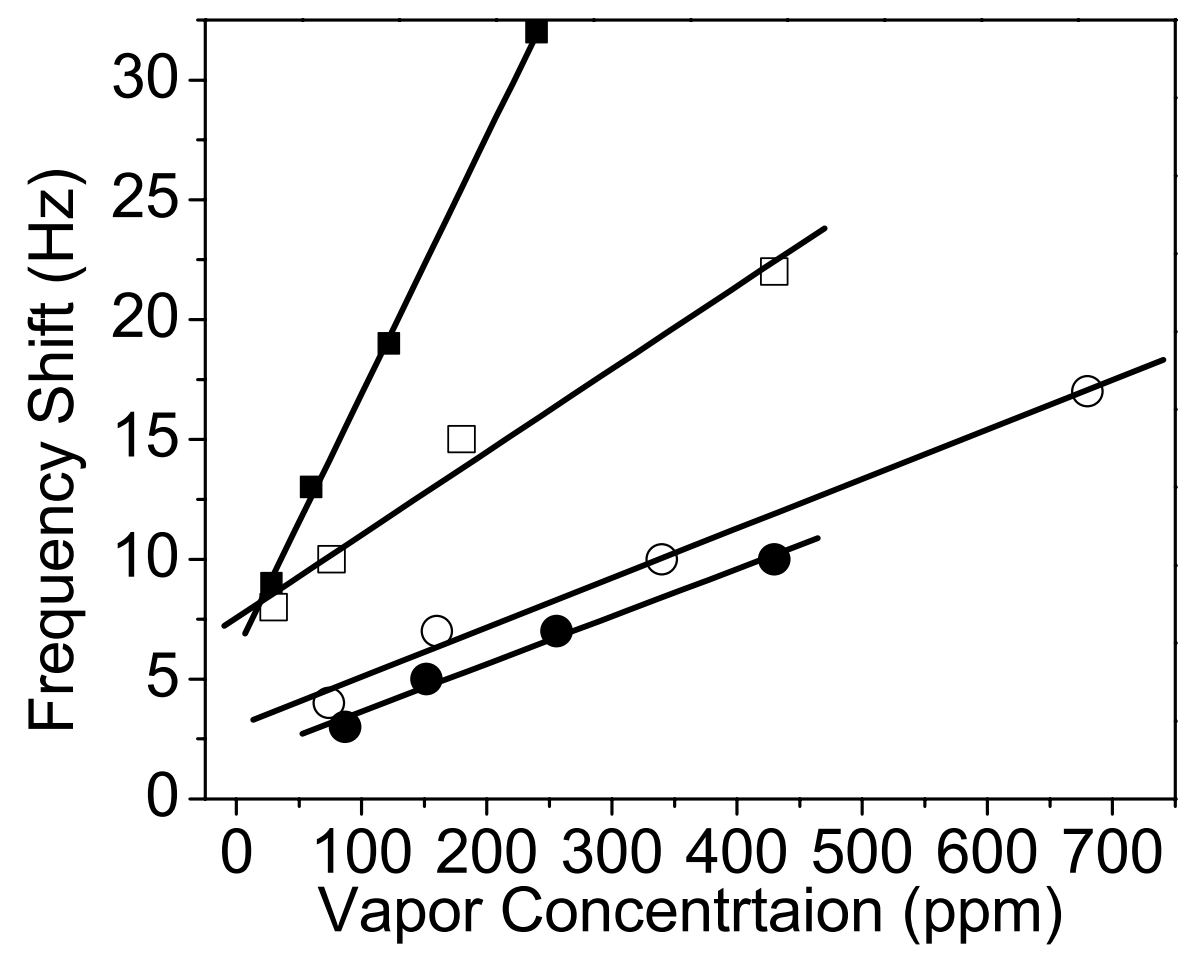

Figure1.20 Imprinted Microsphere Polymer (IMP) calibration curves PIB coating: $2 \mathrm{kHz}$, IMP coating: $3 \mathrm{kHz}$. IMP: methacrylic acid polymer imprinted with hydroquinone.

Solid square : $\mathrm{TOL}, \mathrm{y}=6.17+0.107 \times \mathrm{R}^{2}=0.998, \mathrm{~K}=9,500$;

hollow square: $B E Z, y=7.55+0.0346 x R^{2}=0.982, K=3,600$;

solid circle: HEP, $y=1.67+0.0198 x, R^{2}=0.986, K=1,600$;

hollow circle: $\mathrm{CCl}_{4}, \mathrm{y}=3.02+0.0207 x, \mathrm{R}^{2}=0.992, \mathrm{~K}=1,100$;

RSDs of frequency shifts based on 3 trials are $1.7 \%$ for TOL, $3.2 \%$ for BEZ, $3.8 \%$ for Hep and $4.9 \%$ for $\mathrm{CCl}_{4}$, respectively. 


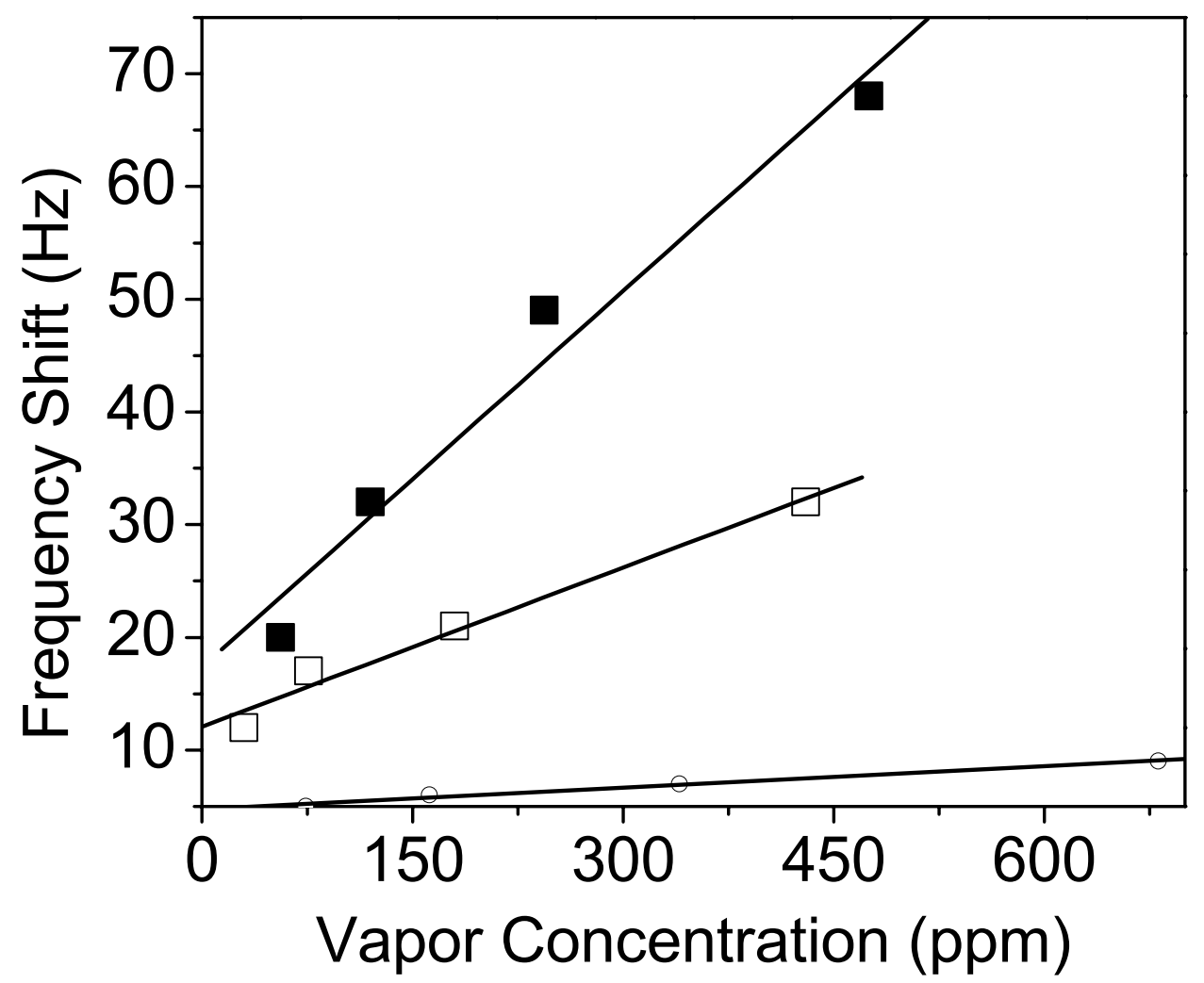

Figure 1.21 Molecularly Imprinted Membrane (MIM) (imprinted with hydroquinone) calibration Curves

QCM coating : $15 \mathrm{kHz}$ coating of $\mathrm{MIM}(\mathrm{HQ})$.

Solid square : $T O L, y=17.3+0.111 \times R^{2}=0.968, K=2,000$

hollow square: $B E Z, y=12.1+0.0471 \times R^{2}=0.980, K=1,000$;

hollow circle: $\mathrm{CCl}_{4,} \mathrm{y}=7.9+0.00182 x, \mathrm{R}^{2}=0.850 ; \mathrm{K}=300$.

RSDs of frequency shifts based on 3 trials are $3.7 \%$ for TOL, $4.4 \%$ for BEZ,

$6.1 \%$ for $\mathrm{CCl}_{4}$, respectively. 


\subsubsection{Coating Thickness}

The Sauerbrey equation indicates that the frequency shift during vapor sorption $\left(\Delta f_{v}\right)$ is linearly related to the amount of coating deposited $\left(\Delta f_{s}\right)$. In Figure 1.22 , frequency shifts for a constant organic vapor concentration are shown with increasing coating amounts of BIP polymer powders applied onto a QCM surface. All data are obtained on one QCM with a fixed amount of PIB layer $(2 \mathrm{kHz})$. After the measurement of frequency shifts is obtained for each of the five different vapors, an additional layer of BIP particles is deposited on the QCM surface by evaporation of the ethanol suspension. The MIP coating thickness is expressed in terms of the change in frequency of the QCM following evaporation of the suspension of the MIP on the QCM surface. The response is not linear, suggesting that greater amounts of MIP powders have difficulty in coupling mechanically to the QCM via the PIB binding layer. Increasing coating thickness also increases the amount of vapor collected at the surface, and hence increases absolute sensitivity. However, the attenuation of surface wave energy by stiff polymer coatings places practical limitations on coating thickness. Attempts to obtain methacrylic polymer coating thicknesses greater than $10 \mathrm{kHz}$ lead to a long-term baseline frequency shift, unstable oscillation and even cessation of oscillation of the QCMs. Also, the overly-thick coatings of MIP on the PIB surface flake off easily, resulting in a decrease in the sensor sensitivity with time. For these reasons, typical coatings of BIP and MIM polymers from $2 \mathrm{kHz}$ to $5 \mathrm{kHz}$ are used in the experiments to evaluate relative sensitivity and selectivity. For acrylonitrile membrane polymers, frequency changes 
upon application of the polymer are often around several dozens of $\mathrm{kHz}$ and the coated QCM shows a much more smooth surface morphology compared to the methacrylic polymers.

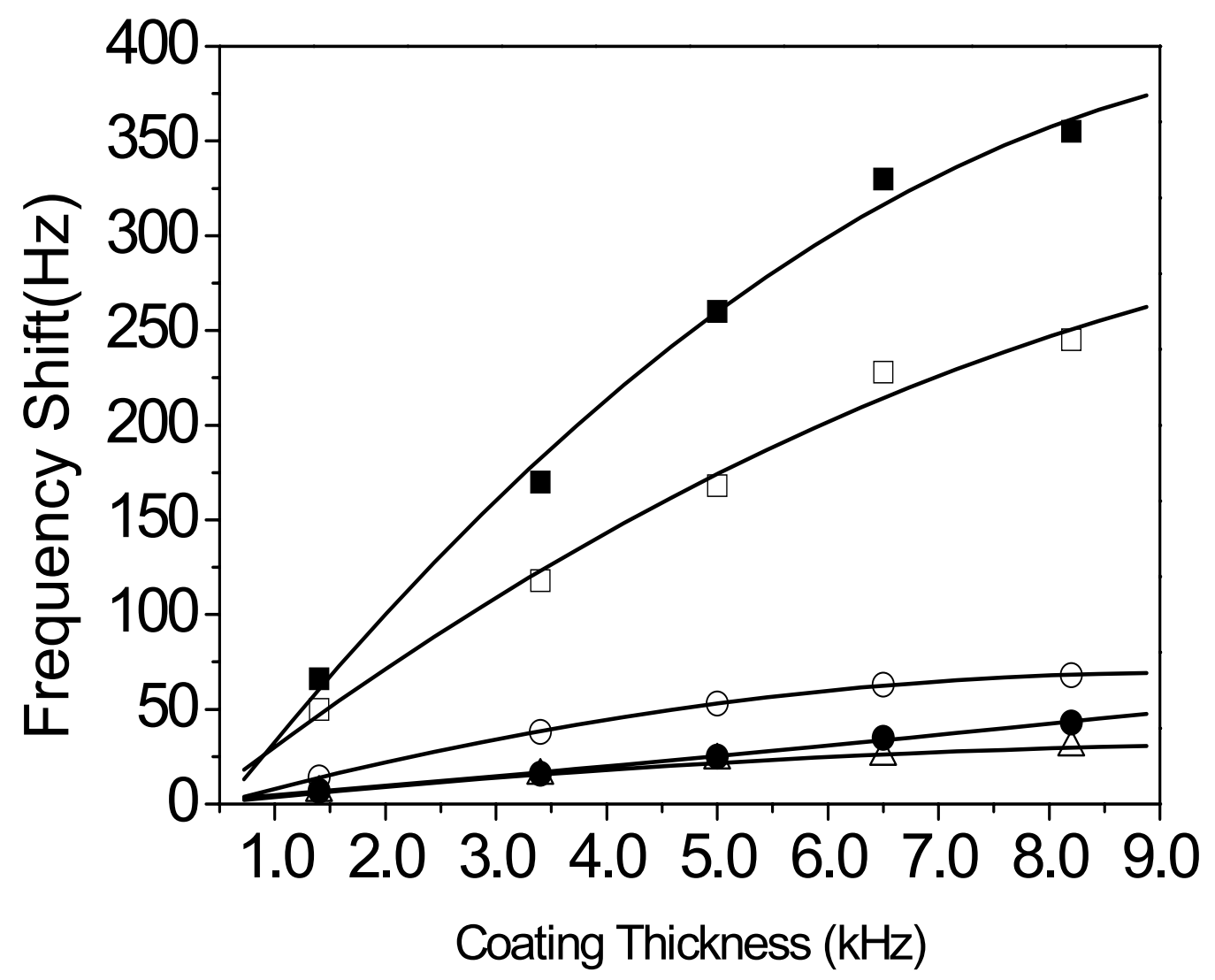

Figure 1.22 QCM frequency responses vs the coating amounts Vapor concentrations: 300 ppm. MIP: block acrylic polymer imprinted with hydroquinone. (Solid square: TCE; hollow square: TOL; hollow circle: BEZ; solid circle: HEP; hollow triangle: $\mathrm{CCl}_{4}$ ). ( Results based on one experiment only) 


\subsubsection{Frequency-time response}

Under a stream of dry air, the QCM crystal oscillates at a base-line value, with the frequency of the coated QCM being lower than that of the reference QCM. Exposure to air containing a vapor which is adsorbed by the coating changes the coated QCM frequency to a lower value. Ideally, the frequency of the reference QCM remains unchanged, and the difference frequency between the two crystals increases. This increase is taken as the sensor's response, $\Delta \mathrm{f}_{\mathrm{v}}$, and is a measure of vapor sorption. After complete desorption of the adsorbed vapor (as indicated by a return to the pre-pulse baseline), the coated QCM is subjected to a second vapor pulse. A typical response curve for three successive vapor exposure is shown in Figure 1.23. $\Delta f_{v}$ varies slightly from the first response pulse with a change of 2 to $4 \%$.

The time-dependent response of QCMs is characteristic of specific vaporsubstrate (MIP) interactions. Frequency-time response profiles of coated QCMs toward vapor pulses are evaluated with respect to rise time constant, decay time constant and reproducibility over multiple vapor pulses. Rise and decay fractions of the response profiles are extracted from the real-time response curves. The data are imported into Corel Quattro Pro for further manipulation. Time constants $(\tau)$ are obtained from fitting the data with the following exponential functions (Equation 1-9, 1-10). The minimized function is the sum of squared difference between the experimental data and the theoretical values at the same time. The optimization parameters for the response data are the baseline value $\left(\mathrm{A}_{0}\right)$ of the response, the relative response value $\left(A_{1}\right)$ and the time constant $(\tau)$. 


$$
\begin{aligned}
& \text { Exponential growth fitting : } y=A_{0}+A_{1}\left(1-e^{-t / \tau}\right) \text {; } \\
& \text { Exponential decay fitting: } y=A_{0}+A_{1} e^{-t / \tau}
\end{aligned}
$$

Figure 1.24 shows a coated QCM sensor responding to different vapor concentrations of toluene. The QCM is coated with $3 \mathrm{kHz}$ bulk imprinted polymer. We observe a good reversibility (sorption and desorption look symmetric) in the realtime response curves. The polymer film responses quickly to TOL vapor. The time constants associated with adsorption are approximately same (10 seconds) for all three different concentrations. As the concentration of TOL vapor is increased, the amount of TOL vapor retained on the polymer film is increased. This is clearly illustrated in Figure 1.24, which shows that both the frequency change and the desorption time constant at higher concentration level are larger than those at relatively low concentration. 


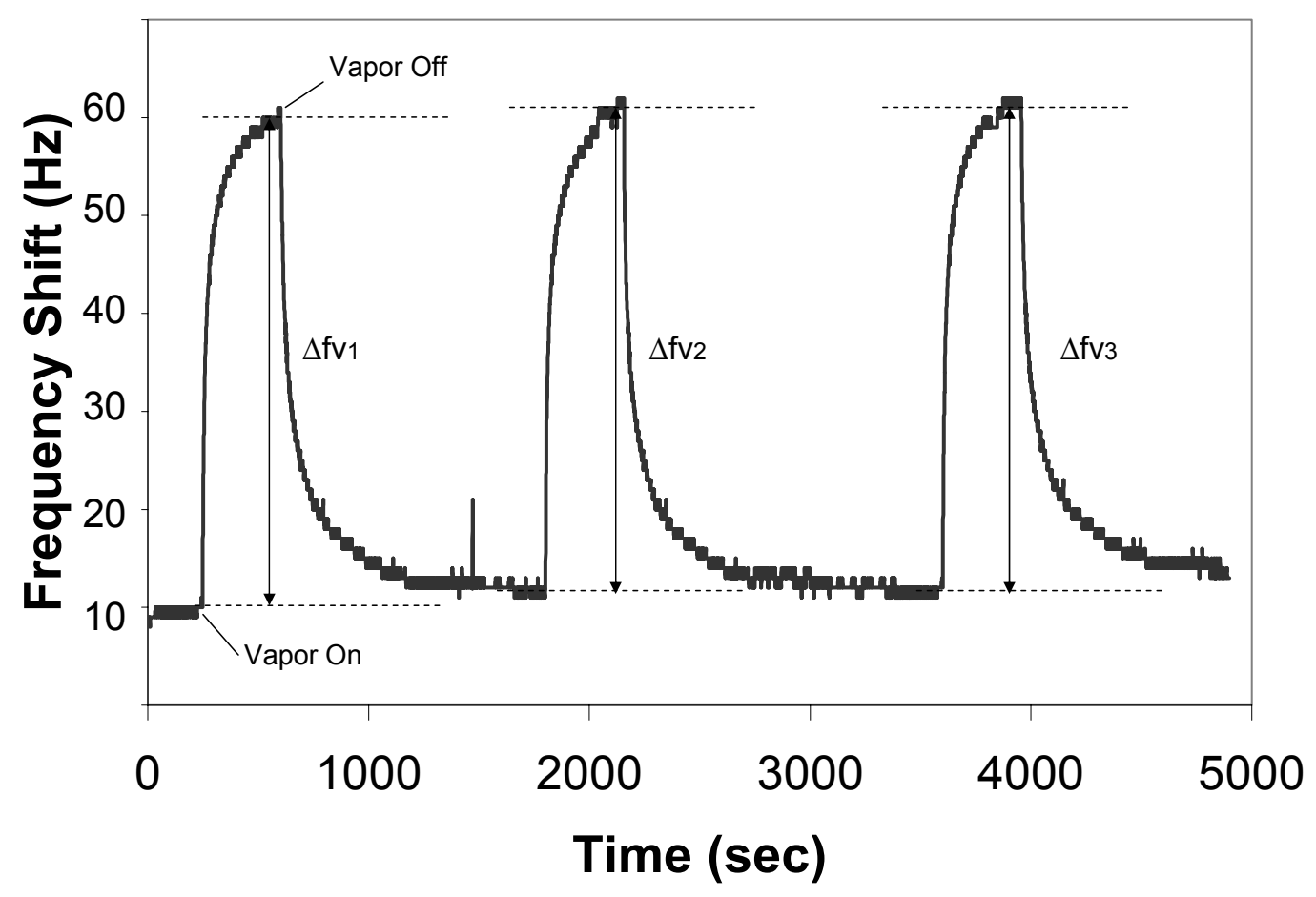

Figure 1.23 A typical real-time frequency response of coated QCM responding to Toluene vapor. Vapor concentration : $120 \mathrm{ppm}$;

QCM coating: 4k PIB plus 2k BIP (HQ).

$\Delta f v 1=50 \mathrm{~Hz}, \Delta f \mathrm{f} 2=48 \mathrm{~Hz}, \Delta \mathrm{fv} 3=51 \mathrm{~Hz}$. 


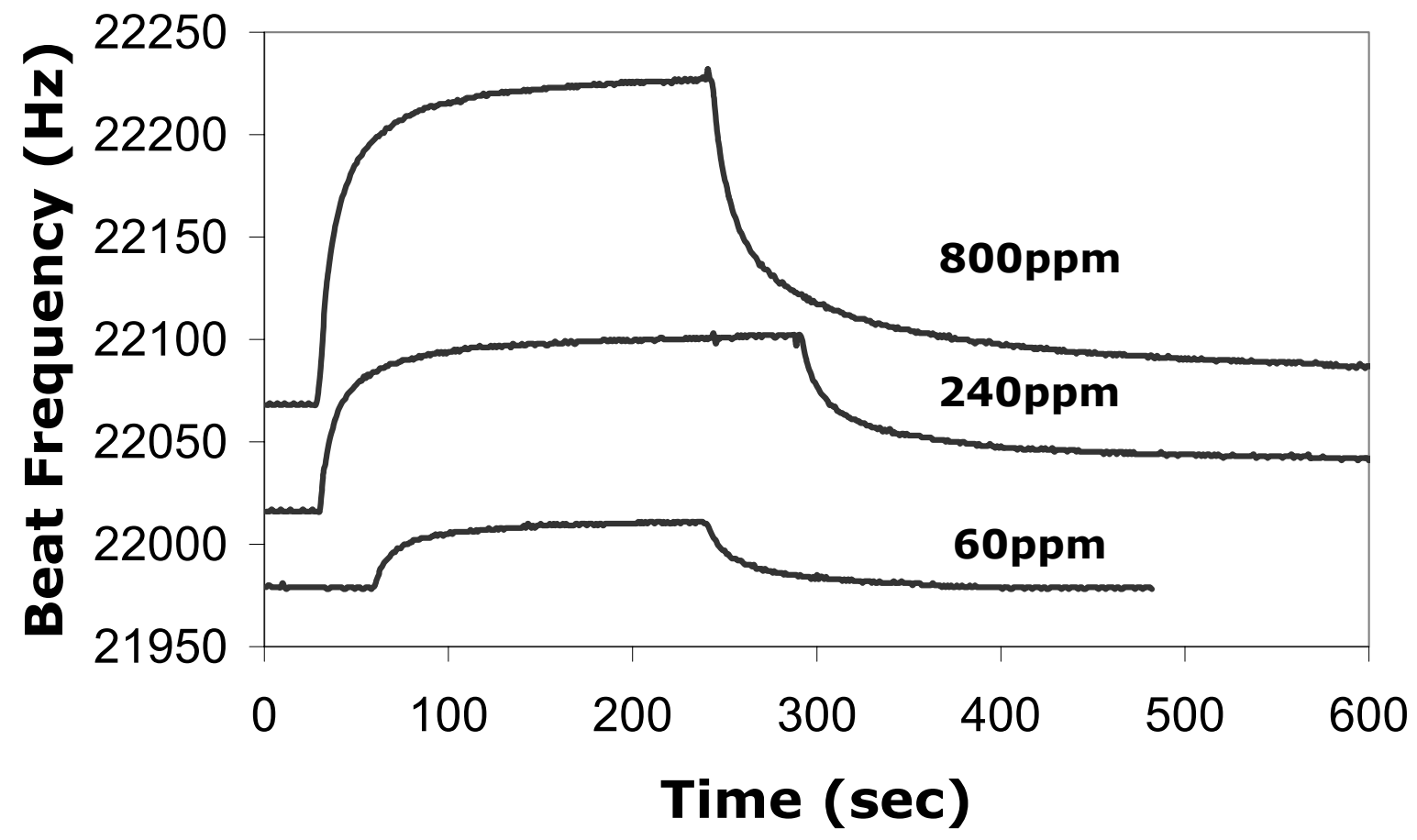

Figure 1.24 Response of Block imprinted polymer (BIP (HQ)) to TOL vapor with a variety concentrations. QCM coating: $2.8 \mathrm{kHz}$ BIP (HQ) plus $2 \mathrm{k} \mathrm{Hz}$ PIB.

Time constants $(\tau)$ for $60 \mathrm{ppm}: \mathrm{T}($ rise $)=9.6 \pm 0.6 \mathrm{sec} ; \mathrm{T}($ decay $)=22 \pm 1.8 \mathrm{sec}$;

Time constants $(\tau)$ for 240 ppm: $T($ rise $)=10 \pm 1.2$ sec; $\tau($ decay $)=31 \pm 3$ sec;

Time constants $(\tau)$ for 800 ppm: $T($ rise $)=11 \pm 1.8 \mathrm{sec} ; T($ decay $)=40 \pm 4.2 \mathrm{sec}$;

RSDs for rise and decay time constants are based on 3 trials (successive pulses). 
The response behaviors of three types of synthesized MIPs and their corresponding control polymers are investigated toward analytes with different molecular sizes and shapes at a chosen vapor concentration. Typical frequency-time plots of QCM sensor coated with BIP(HQ), IMP(HQ) and MIM(HQ) responding to different organic vapors at $300 \mathrm{ppm}$ concentration are shown in Figure 1.25, Figure 1.26 and Figure 1.27, respectively. The time constants derived from the rise and decay fractions are given in Table 1.4 .

In general, thinner MIP films exhibit more rapid responses to vapor pulses while thicker MIP films yield somewhat slower rise and decay times. Qualitatively, all MIP coatings equilibrate with increasing vapor concentrations in about a minute. However, for each type of imprinted polymer, the difference among the responses to all vapors is quite clear. The block imprinted polymer exhibits a very quick uptake behaviors for all vapors. Microsphere polymers and membrane polymers show the similar uptake behaviors for TCE, heptanes and $\mathrm{CCl}_{4}$, but somewhat slower for toluene and benzene during the adsorption.

The slow release behavior of TOL is observed for all polymers imprinted with HQ during desorption, especially for the BIP polymer. The rather slow decay times of TOL suggest a particularly strong adsorption of toluene molecules on the imprinted polymer and is evident for an imprinting effect. The decay time constants of heptanes and $\mathrm{CCl}_{4}$ for all imprinted polymers are in the range of 5 to 15 seconds. The noticeable faster uptake and release rates indicate the presence of weaker adsorption among the MIPs and these two vapors. The weaker binding permits the vapor molecules to diffuse more rapidly through the matrix. This is analogous to the longer 
retention times of template molecules on MIPs. In simple kinetic terms, the ratio of the desorption rate constant to the adsorption rate constant is equal to the partition coefficient. The greater desorption time constants for TOL and BEZ on the BIP polymer is therefore consistent with the partition coefficients noted earlier (Section 3.2.2)

The selective responses of the QCM sensor coated with BIP particles via cellulose binder is also displayed in Figure 1.28. All of the analytes tested exhibit reversible adsorption/desorption behaviors in this coating. The frequency changes associated with TOL, BEZ and TCE are much higher than that of $\mathrm{CCl}_{4}$. Since cellulose additives contribute very little to vapor adsorption, the resulting frequency changes are directly related to vapor adsorptions of BIP particles. 


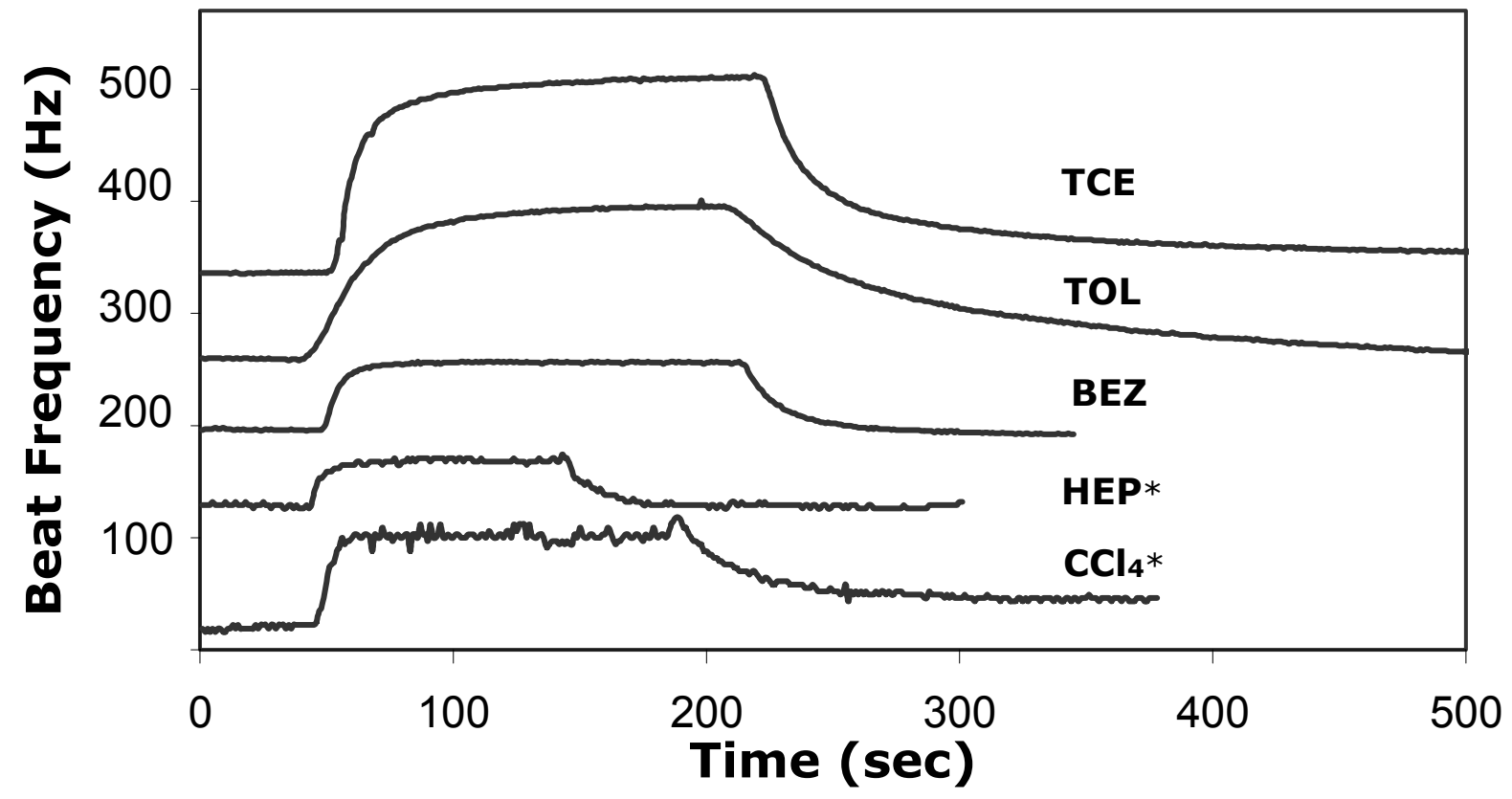

Figure 1.25 Typical real-time response curves of QCM coated with BIP Vapor concentrations: $300 \mathrm{ppm}$. MIP: block acrylic polymer imprinted with hydroquinone; QCM coating: 4 kHz PIB plus $4.8 \mathrm{kHz}$ polymer layer. *Plots for Heptanes and $\mathrm{CCl}_{4}$ are enlarged by a factor of 3 


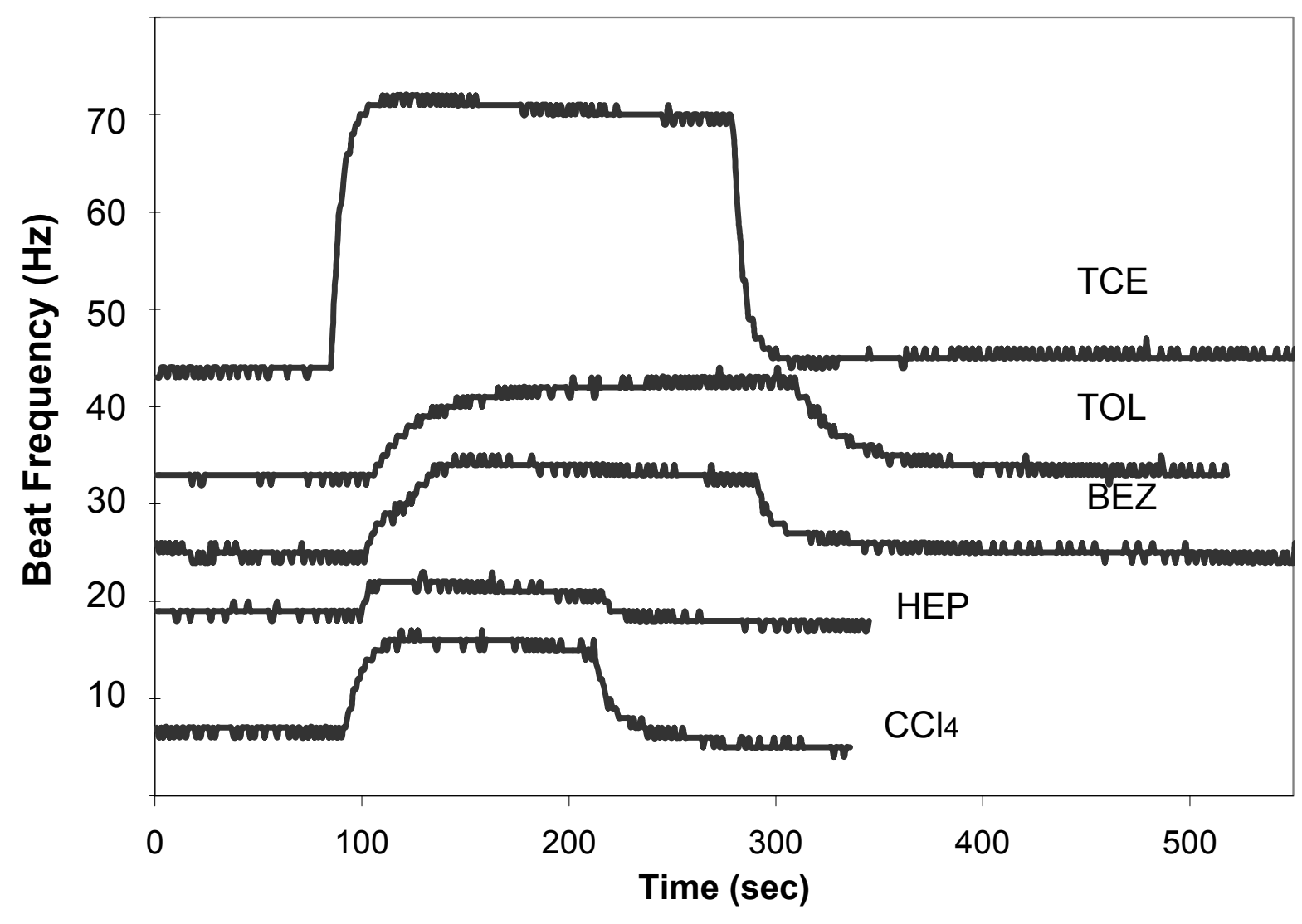

Figure 1.26 Typical real-time response curves of QCM coated with IMP Vapor concentrations: 300 ppm. IMP : microsphere polymer imprinted with hydroquinone; QCM coating: $4 \mathrm{kHz}$ PIB plus $4.8 \mathrm{kHz}$ polymer layer 


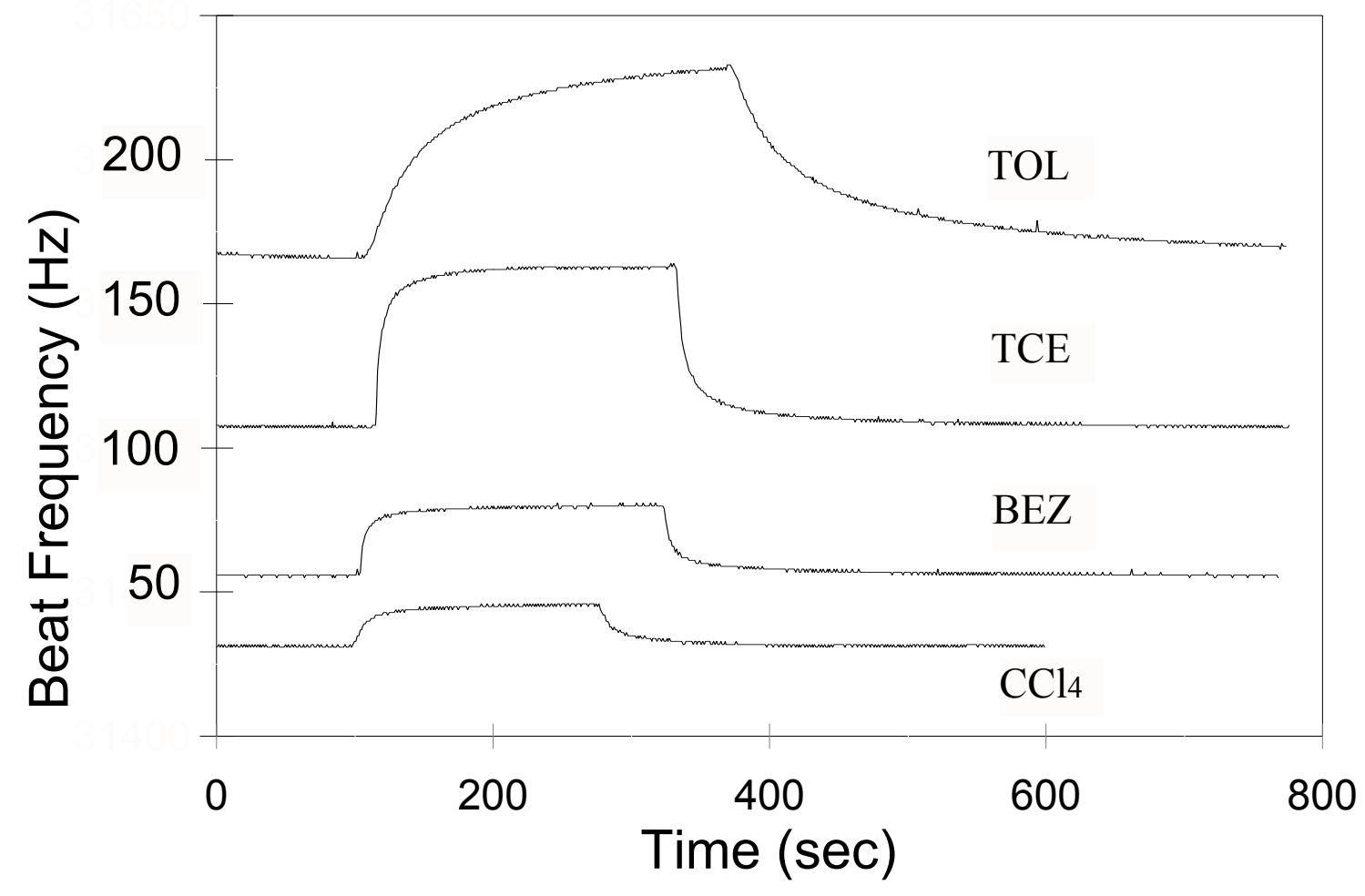

Figure 1.27 Typical real-time response curves of QCM coated with MIM. Vapor concentrations: $300 \mathrm{ppm}$. MIM : membrane polymer imprinted with hydroquinone; Coating amount : $28 \mathrm{kHz}$ 
Table 1.4 Time constants of MIP adsorption and desorption responses to vapor pulses*

\begin{tabular}{c|c|c|c|c|c|c}
\hline \multirow{2}{*}{ MIP } & Time & \multicolumn{5}{c}{ Vapors } \\
\cline { 3 - 7 } & constant & TCE & TOL & BEZ & CCl $_{4}$ & HEP \\
\hline \multirow{3}{*}{ BIP } & $\begin{array}{c}\text { Adsorption } \\
\text { (sec) }\end{array}$ & $9.0 \pm 1.1$ & $12 \pm 1.5$ & $5.3 \pm 0.7$ & $8.7 \pm 1.6$ & $8.0 \pm 1.2$ \\
\cline { 2 - 7 } & $\begin{array}{c}\text { Desorption } \\
\text { (sec) }\end{array}$ & $28 \pm 2.9$ & $90 \pm 5.9$ & $18 \pm 2.4$ & $15 \pm 2.1$ & $11 \pm 3.2$ \\
\hline \multirow{3}{*}{ IMP } & $\begin{array}{c}\text { Adsorption } \\
\text { (sec) }\end{array}$ & $6.0 \pm 0.8$ & $22 \pm 1.7$ & $25 \pm 2.3$ & $4.1 \pm 0.8$ & $11 \pm 1.2$ \\
\cline { 2 - 7 } & $\begin{array}{c}\text { Desorption } \\
\text { (sec) }\end{array}$ & $6.2 \pm 1.2$ & $22 \pm 1.5$ & $16 \pm 1.7$ & $6.7 \pm 2.0$ & $7.1 \pm 1.9$ \\
\hline \multirow{3}{*}{ MIM } & $\begin{array}{c}\text { Adsorption } \\
\text { (sec) }\end{array}$ & $5.6 \pm 1.0$ & $30 \pm 2.6$ & $8.2 \pm 1.9$ & $5.3 \pm 0.8$ & N/A \\
\cline { 2 - 7 } & $\begin{array}{c}\text { Desorption } \\
\text { (sec) }\end{array}$ & $11 \pm 2.0$ & $28 \pm 1.9$ & $10 \pm 1.8$ & $6.9 \pm 1.0$ & N/A \\
\hline
\end{tabular}

*Time constants were obtained by fitting the rising and decaying responses to exponential functions. RSDs for rise and decay time constants are based on 3 trials (successive pulses). 


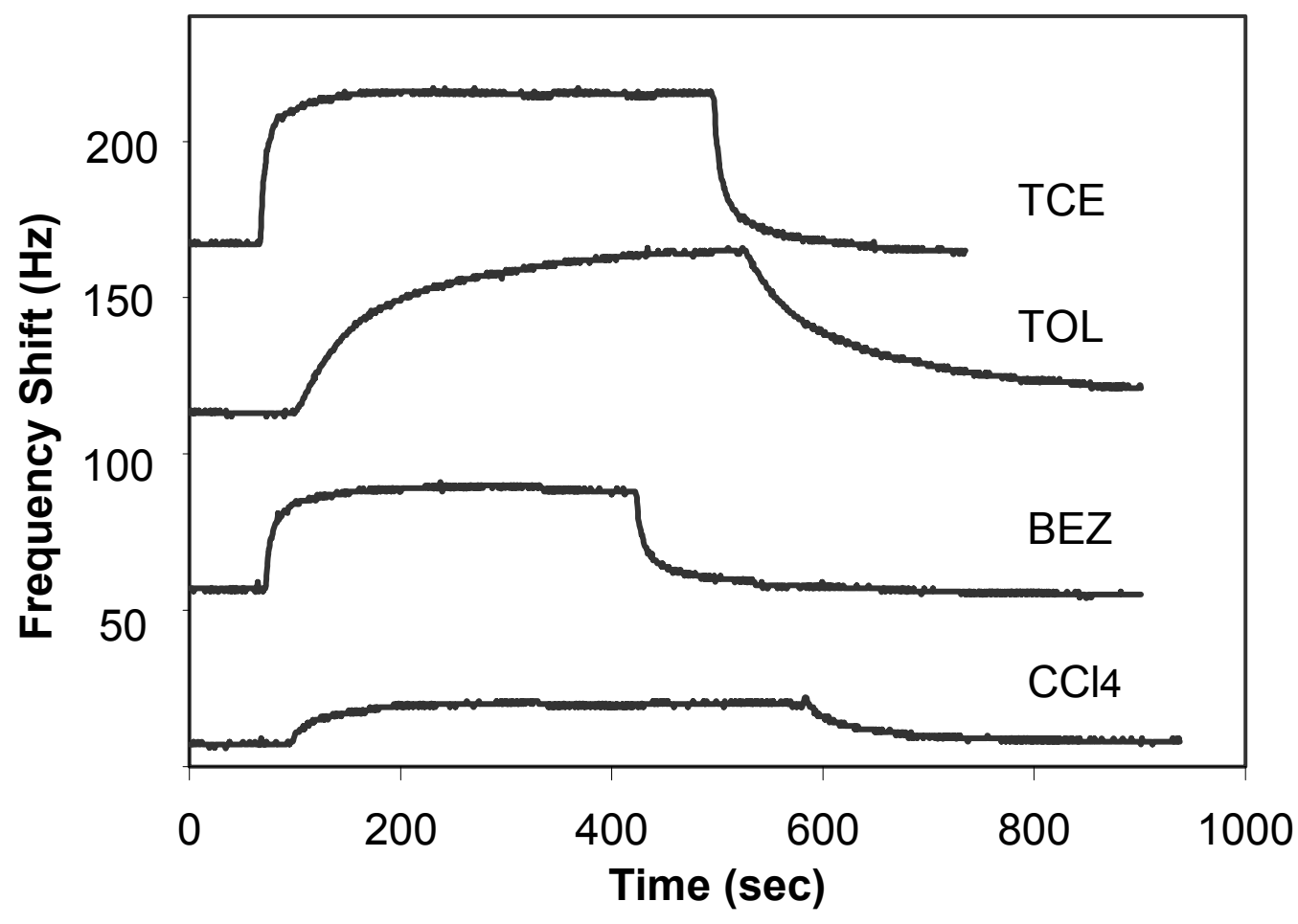

Figure 1.28 Real-time response curve of QCM coated with BIP via cellulose binder, total coating on QCM: $5 \mathrm{kHz}$

Time constants $(\tau)$ for TCE : $T($ rise $)=8 \pm 0.9$ sec; $T($ decay $)=17 \pm 2.0$ sec;

Time constants $(\tau$ ) for TOL: $T($ rise $)=58 \pm 6.0$ sec; $T($ decay $)=90 \pm 8.7$ sec;

Time constants ( $\mathrm{\tau}$ ) for BEZ : $\mathrm{T}($ rise $)=9 \pm 1.3 \mathrm{sec}$; $\mathrm{T}($ decay $)=20 \pm 2.4 \mathrm{sec}$;

Time constants $(\tau)$ for $\mathrm{CCl}_{4}: T($ rise $)=15 \pm 1.8 \mathrm{sec} ; \mathrm{T}($ decay $)=46 \pm 3.2 \mathrm{sec}$

RSDs for rise and decay time constants are based on 3 trials (successive pulses). 
The imprinting effect becomes clearer when frequency-time response curves for toluene (Figure 1.29), heptanes (Figure 1.30) and $\mathrm{CCl}_{4}$ (Figure 1.31) are compared. In each figure are included the response curves for a block polymer imprinted with hydroquinone, a control polymer (non-imprinted) and/or a PIB coating. Obviously, a much higher response for TOL is observed with the imprinted polymer. For heptanes and $\mathrm{CCl}_{4}$, the response behaviors to these compounds using the control polymer are similar to those observed for the imprinted polymer with the close frequency shifts. The rise times and decay times are very similar for heptanes and $\mathrm{CCl}_{4}$ in imprinted and non-imprinted polymers. In contrast, the decay time for toluene vapor pulses lengthen substantially in the imprinted polymers. 


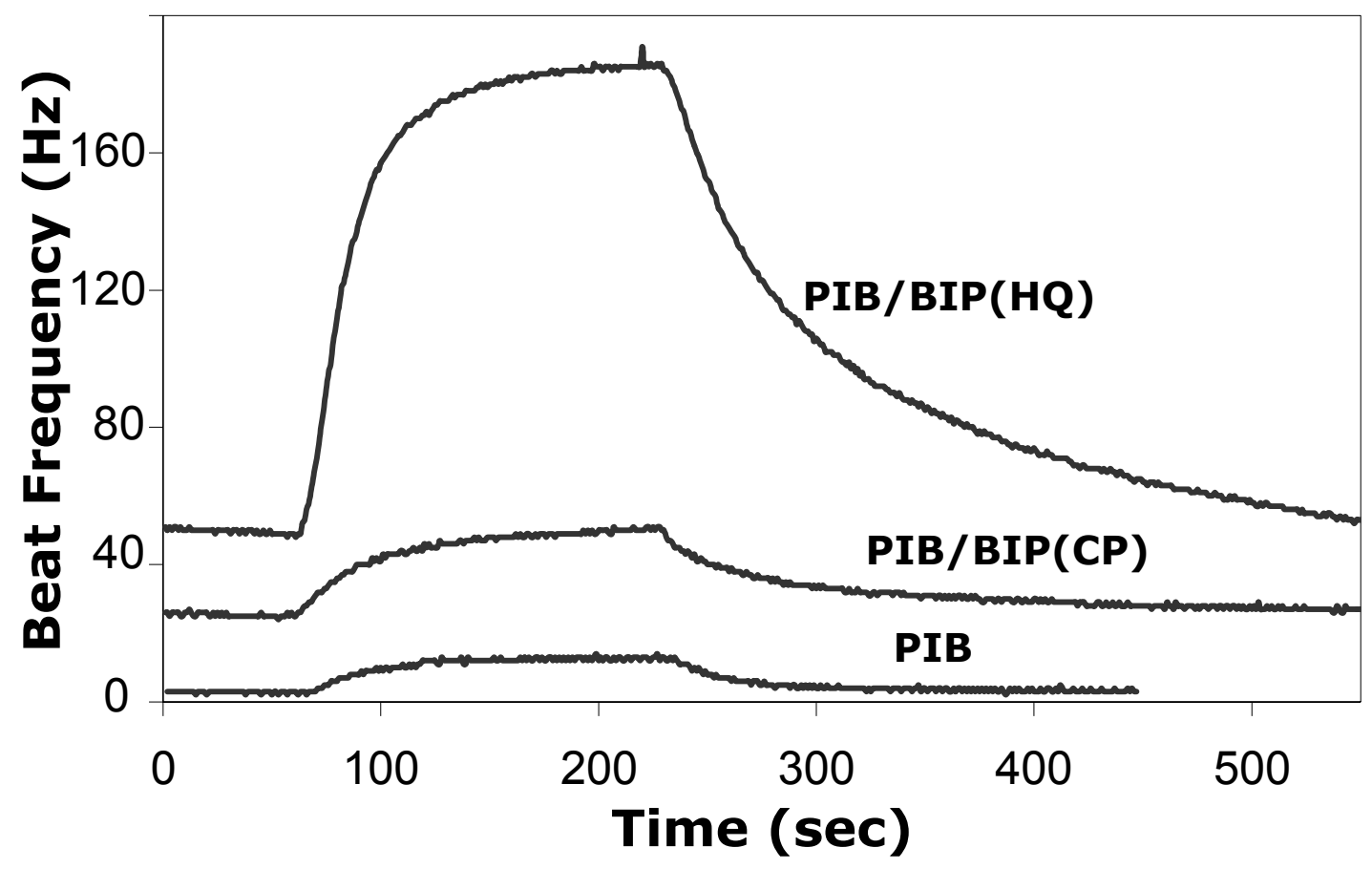

Figure 1.29 Responses of imprinted and control polymer layers to toluene vapor. Vapor concentration: 300 ppm; Coating: 2 kHz PIB layer plus 2 kHz BIP (acrylic acid copolymer); PIB coating: 2 kHz.

Rise time constants: $(B I P)=13 \pm 1.5 \mathrm{sec} ;(C P)=15 \pm 2.0 \mathrm{sec} ;(P I B)=15 \pm 1.9 \mathrm{sec}$. Decay time constants: $(B I P)=90 \pm 5.8 \mathrm{sec} ;(C P)=25 \pm 3.1 \mathrm{sec} ;(P I B)=20 \pm 2.1 \mathrm{sec}$. RSDs for rise and decay time constants are based on 3 trials (successive pulses). 


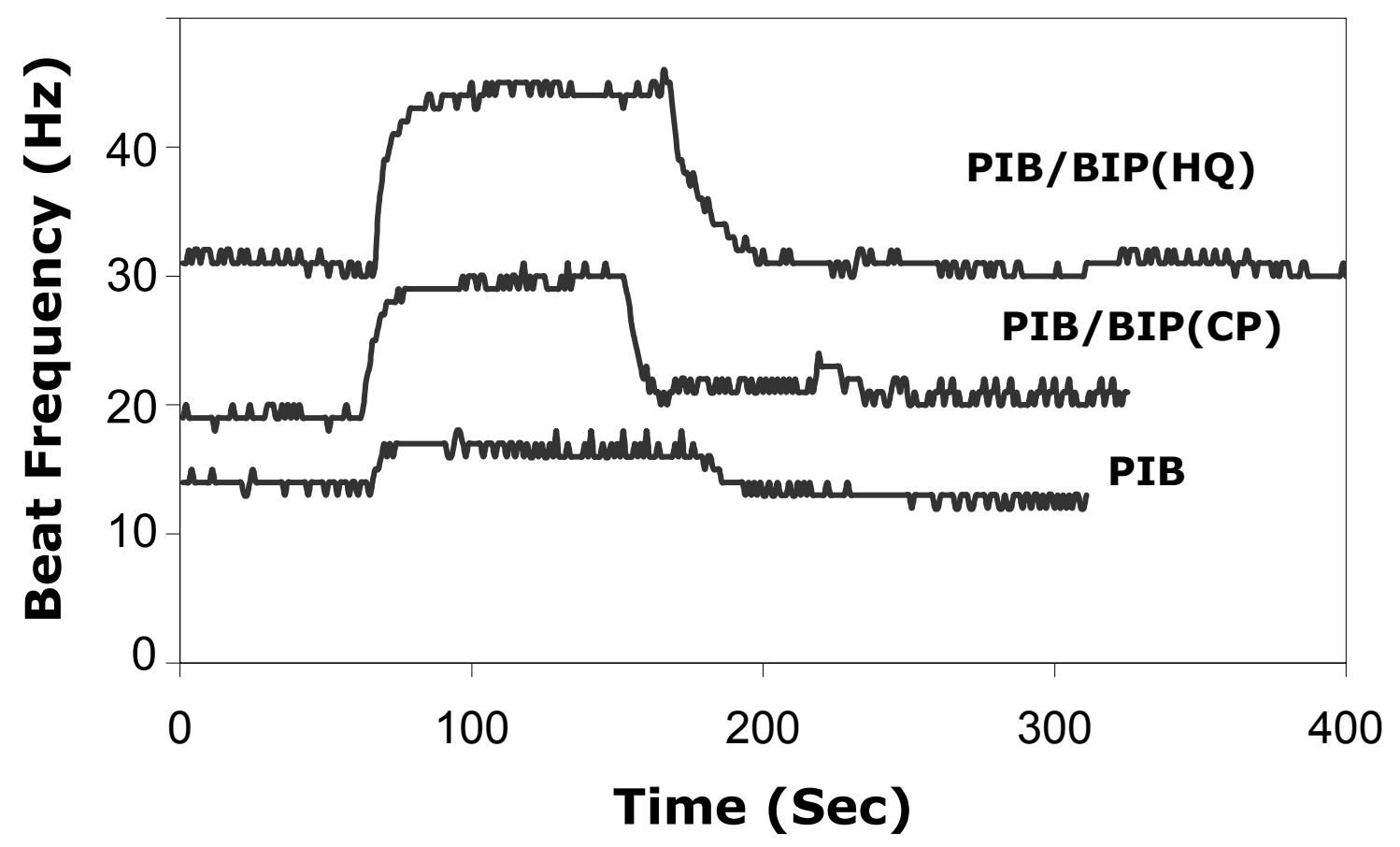

Figure 1.30 Responses of imprinted and control polymer layers to heptanes vapor.

Vapor concentration: 300 ppm. Coating: 2 kHz PIB layer plus 2 kHz BIP(acrylic acid copolymer), PIB coating: 2 kHz.

Rise time constants: $(B I P)=4.1 \pm 1.2 \mathrm{sec} ;(C P)=5.6 \pm 1.6 \mathrm{sec} ;(P I B)=2.1 \pm 0.8 \mathrm{sec}$.

Decay time constants: $(B I P)=11 \pm 3.1 \mathrm{sec} ;(C P)=8.3 \pm 2.3 \mathrm{sec} ;(P I B)=10 \pm 1.1 \mathrm{sec}$.

RSDs for rise and decay time constants are based on 3 trials (successive pulses). 


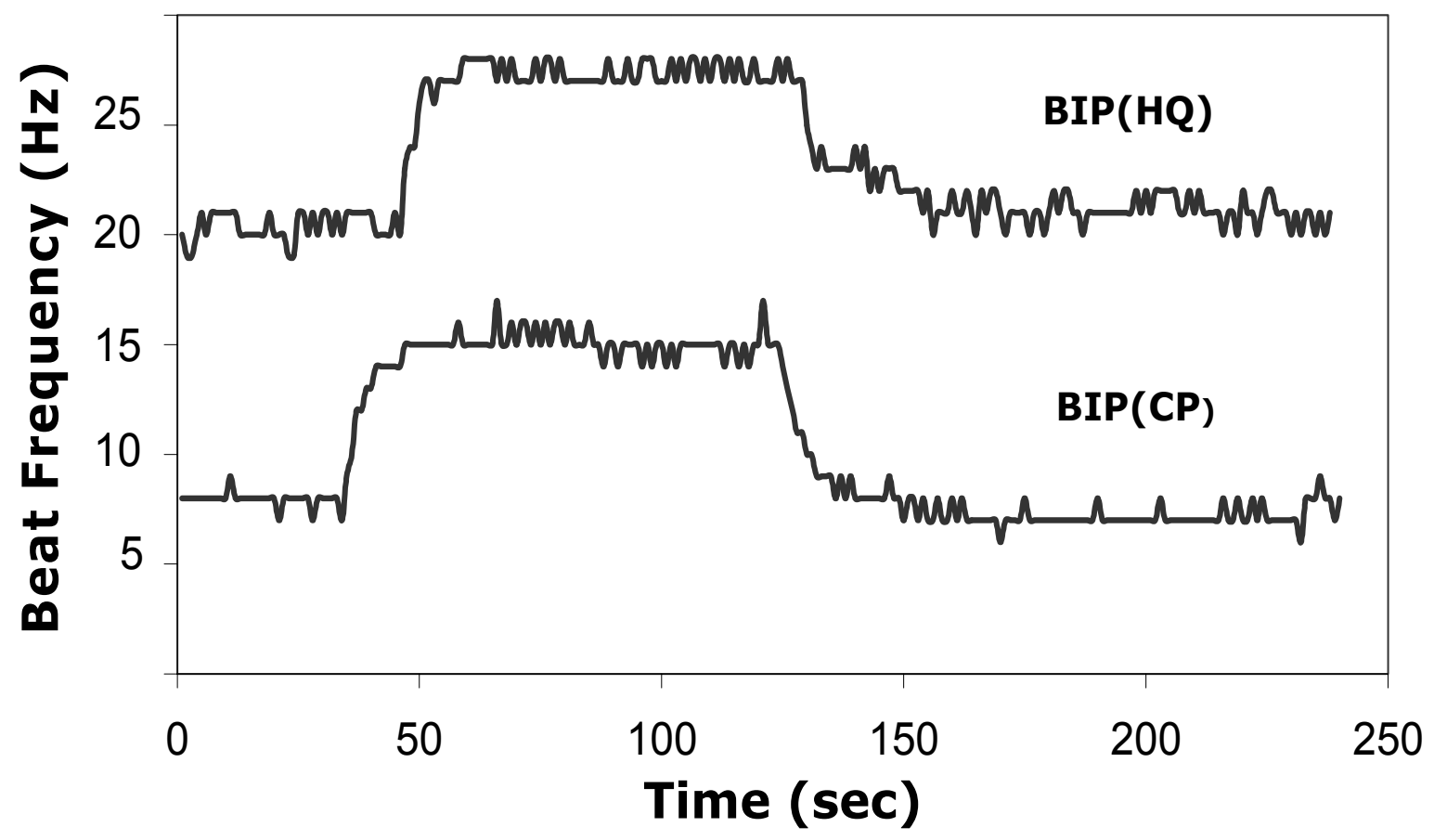

Figure 1.31 Responses of imprinted and control polymer layers to $\mathrm{CCl}_{4}$ vapor. Vapor concentration: 300 ppm. Coating: 2 kHz PIB layer plus 2 kHz BIP(acrylic acid copolymer). Rise time constants: $(B I P)=4.7 \pm 0.6 \mathrm{sec} ;(C P)=4.6 \pm 0.9 \mathrm{sec}$. Decay time constants: $(B I P)=13 \pm 2.2 \mathrm{sec} ;(C P)=9.2 \pm 1.0 \mathrm{sec}$.

RSDs for rise and decay time constants are based on 3 trials (successive pulses). 


\subsubsection{Comparison of Partition Coefficients}

As mentioned previously, the partition coefficient is an index of the amount of sorbed gas molecules in the polymer matrix. A large value of $\mathrm{K}$ implies a greater sensitivity of the coating. Patterns of $\mathrm{K}$ are indicators of the selectivity exhibited by the film. The partition coefficients calculated from the frequency responses of the polymer-coated QCM sensor toward vapors at one fixed vapor concentration are systematically compared in Figure 1.32, Figure 1.33 and Figure 1.34. All three sets

of imprinted polymers are examined. The apparent partition coefficients are obtained from the steady-state QCM sensor response at sorption equilibrium and determined by a single point calibration. For QCM used in our experiments, frequency changes are stable within $\pm 1 \mathrm{~Hz}$ and the corresponding uncertainties of calculated partition coefficients are $\pm 300, \pm 210, \pm 350, \pm 30$ and \pm 180 in these cases for TOL, TCE, BEZ, $\mathrm{HEP}$ and $\mathrm{CCl}_{4}$, respectively.

The imprinting effect is immediately obvious in each set of polymers. Polymers imprinted with hydroquinone and phenol exhibit substantially higher partition coefficients for the planar molecules (trichloroethylene, toluene and benzene) than the non-imprinted polymers (control polymers). These results imply that superior selectivity is present in the imprinted polymers. Differences in the partition coefficients for heptanes and carbon tetrachloride between imprinted and nonimprinted polymers are less marked. Polymers imprinted with phenol in all sets exhibit lower partition coefficients compared with those polymer imprinted with hydroquinone, but they still yield significantly higher partition coefficients for planar analytes than the control polymers. 
In Figure 1.32, BIP(HQ) shows the highest partition coefficients with respect to planar molecules (trichloroethylene, toluene and benzene), and the $\mathrm{K}$ values are about one order of magnitude greater than the $\mathrm{K}$ values for heptanes and $\mathrm{CCl}_{4}$. Taking into account of the experimental uncertainty, the selectivity pattern of BIP(HQ) can be expressed in the following order:

$\mathrm{TOL}>\mathrm{TCE} \geq \mathrm{BEZ}>>\mathrm{HEP} \geq \mathrm{CCl}_{4}$

A similar pattern is also found for the BIP polymer imprinted with phenol (BIP(P)). The same pattern of relative partition coefficients are observed for IMP(HQ), IMP(P), $\operatorname{MIM}(\mathrm{HQ})$ and $\mathrm{MIM}(\mathrm{P})$ polymers as well.

Significant higher apparent partition coefficients are observed in Figure 1.35. In this case, block-imprinted polymer particles are deposited onto QCM surface directly without any glue additives. Due to poor adhesion, small frequency changes $\left(\Delta f_{\mathrm{s}}, \sim 1 \mathrm{kHz}\right)$ are obtained for each coating. Because the value of $\Delta f_{\mathrm{s}}$ is doubtful, these results have limited value (i.e, the calculated partition coefficients have a large uncertainty) and can only be used for single comparison with each coating. However, the bar chart suggests that imprinted polymers exhibit much greater adsorption toward TCE, TOL and BEZ vapor than toward $\mathrm{CCl}_{4}$. 


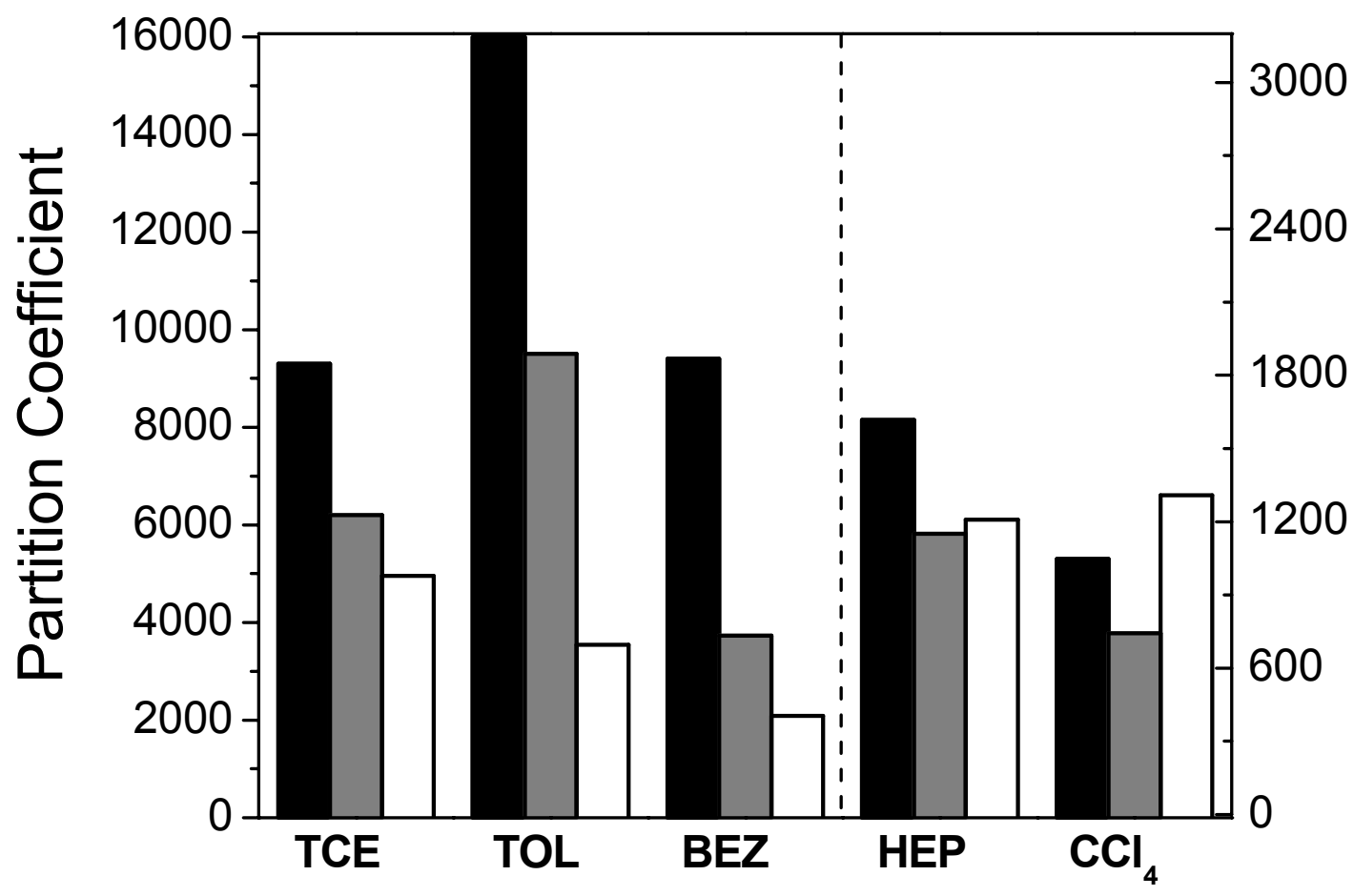

Figure 1.32 Selectivity pattern of bulk imprinted polymers (BIP) to organic vapors. $K$ Values of TCE, TOL and BEZ are scaled by the left $y$ axis, $K$ values of HEP and $\mathrm{CCl}_{4}$ are scaled by the right y axis. Polymers are imprinted with different templates (solid black bar: imprinted with hydroquinone; gray bar: imprinted with phenol; hollow bar: no imprinting).

QCM coating: $2 \mathrm{kHz}$ PIB coating plus $\sim 2 \mathrm{kHz}$ polymer coating. Vapor concentrations: $300 \mathrm{ppm}$. BIP: methacrylic acid copolymer. RSDs of $K$ values based on 3 trials are in the range of $1 \sim 5 \%$ 


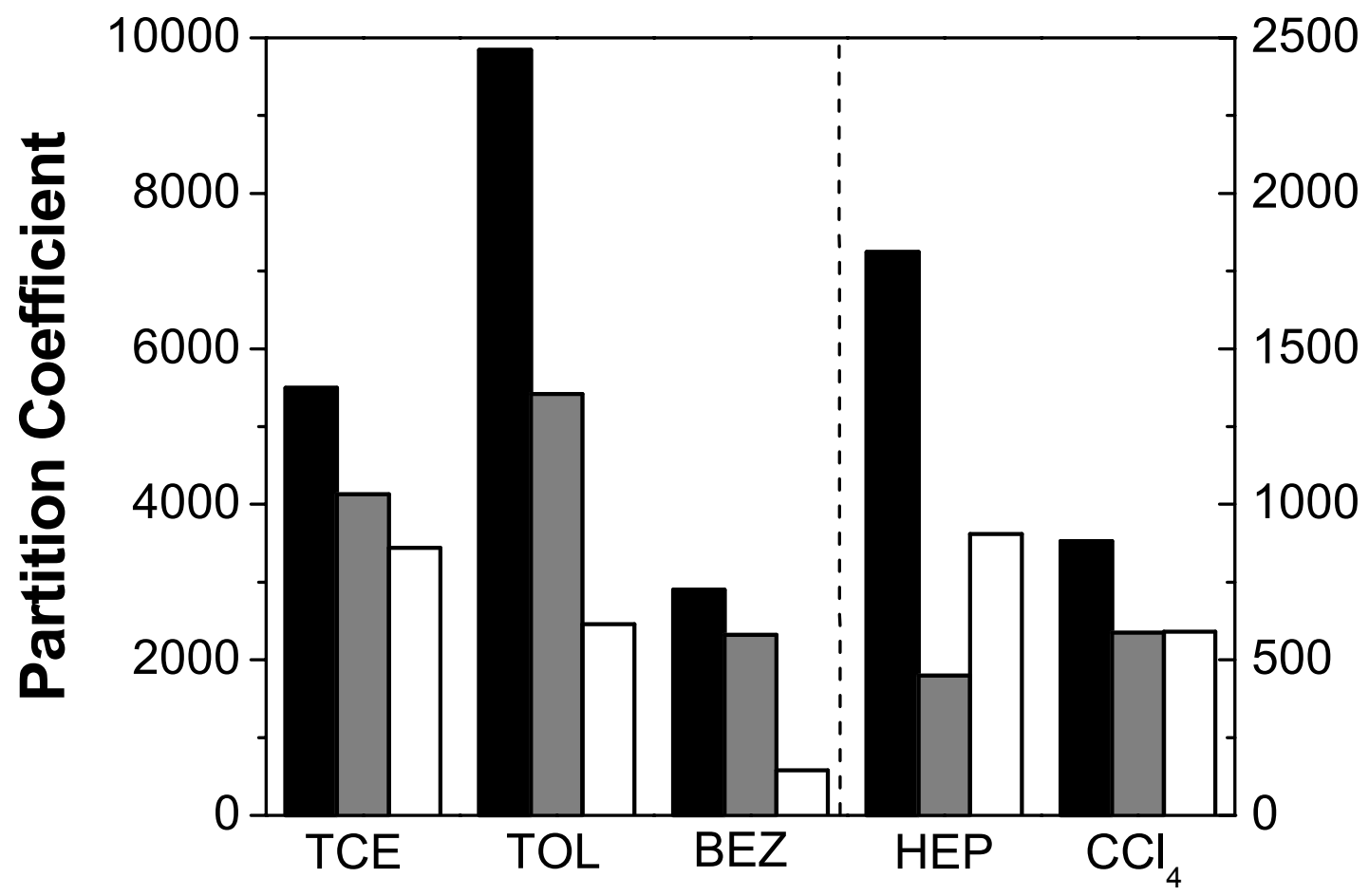

Figure 1.33 Selectivity pattern of imprinted micro-sphere polymers (IMP) to organic vapors.

$K$ Values of TCE, TOL and BEZ are scaled by the left $y$ axis, $K$ values of HEP and $\mathrm{CCl}_{4}$ are scaled by the right y axis. Polymers are imprinted with different templates (solid black bar: imprinted with hydroquinone; gray bar: imprinted with phenol; hollow bar: no imprinting). QCM coating: $2 \mathrm{kHz}$ PIB coating plus $\sim 2 \mathrm{kHz}$ polymer coating. Vapor concentrations: 300 ppm.

IMP: methacrylic acid copolymer.

RSDs of $K$ values based on 3 trials are in the range of $2 \sim 6 \%$ 


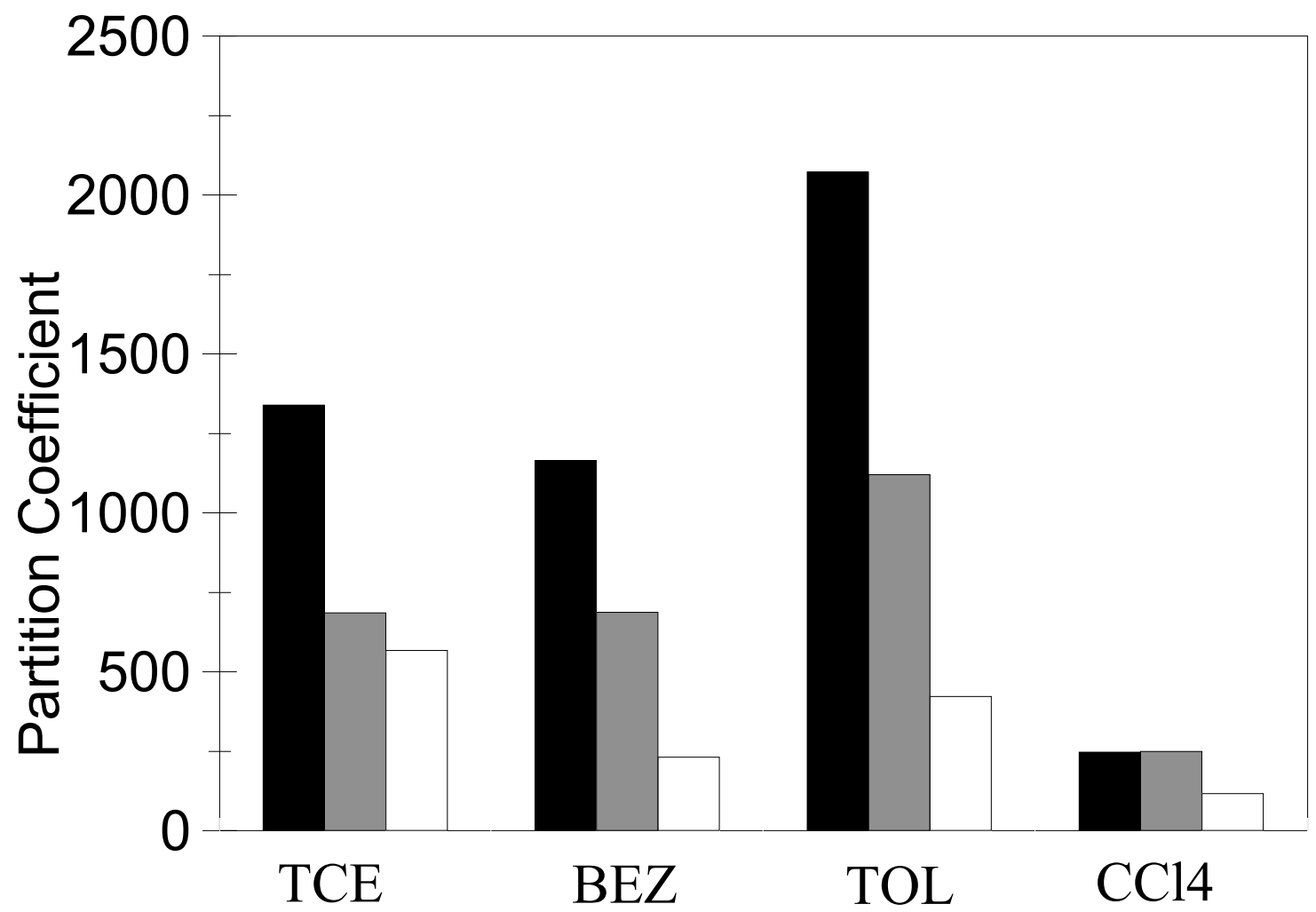

Figure 1.34 Selectivity pattern of membrane imprinted polymers (MIM) to organic vapors. QCM coating: $28 \mathrm{kHz}$. Vapor concentrations: 300 ppm. Polymers are imprinted with different templates (solid black bar: imprinted with hydroquinone; gray bar: imprinted with phenol; hollow bar: no imprinting.

RSDs of $K$ values based on 3 trials are in the range of $3 \sim 7 \%$ 


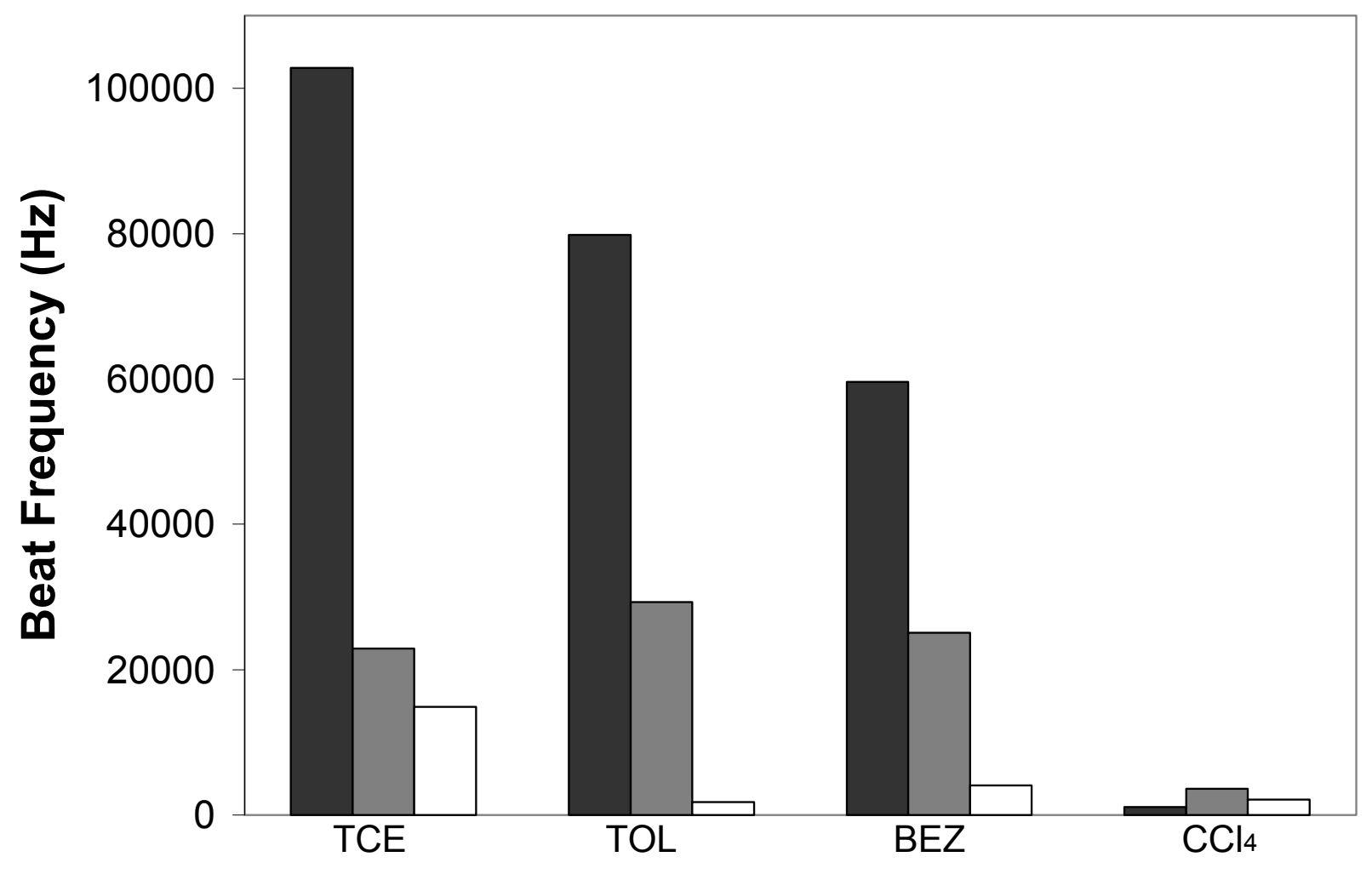

Figure 1.35 BIP selective pattern without PIB additive layer. QCM coating: $1 \mathrm{kHz}$ Polymers are imprinted with different templates (solid black bar: imprinted with hydroquinone; gray bar: imprinted with phenol; hollow bar: no imprinting. RSDs of $K$ values based on 3 trials are in the range of $7 \sim 18 \%$. 
The improved selectivity of the imprinted polymers is better assessed by normalizing the apparent partition coefficients of the MIPs with respect to partition coefficients for PIB. PIB is a fairly stable polymer and has been widely discussed as a coating material for QCM sensors [55]. Grate et al [55] have shown that the log of partition coefficients of soft polymers like PIB scale linearly with respect to the partition coefficients between water and hexadecane. For a homologous series (e.g. benzene, toluene), $\log \mathrm{K}$ scales linearly with the boiling point of the organic substance. Selectivity then can be defined as a deviation from the pattern of $\mathrm{K}$ values for PIB. In other words, if K for PIB represents non-selective intermolecule interaction between the analyte molecule and the polymer, then normalization of $\mathrm{K}$ values for imprinted polymers relative to $\mathrm{K}$ values for PIB should emphasize selectivity, such as the selective interaction between an analyte molecule and an imprint cavity of the right size and shape. QCMs coated with PIB are subjected to the same vapor analytes at the same vapor concentrations. The partition coefficient values of PIB are summarized in Table 1.5.

Table 1.6 contains the normalized partition coefficient values of each of polymer coatings with respect to the partition coefficient of a PIB coating for all tested vapors. RSD for the normalized $k$ values is roughly $10 \%$. Normalized partition coefficients for the non-imprinted polymers generally are approximately the same for the planar (TOL, BEZ) vs. the nonplanar (HEP and $\mathrm{CCl}_{4}$ )analytes, or slightly higher (TCE) for the nonplanar analytes. The block imprinted polymers exhibit substantially larger normalized partition coefficients for the planar analytes relative to the non 
planar analytes, especially when the template molecule is hydroquinone and the functional monomer is acrylic acid (Table $1.6 \mathrm{~b}$ ).

Previous work on molecularly imprinted polymers has shown that two factors contribute to the specificity of the sorption: (i) the cavity shape; and (ii) the presence of chemical groups in the cavity which complement the groups on any potential sorbate ( e.g. the original template) [32]. In our work, the project is to sense nonfunctional aromatic compounds, and it is of interest to observe that the sensitivity of the imprinted polymers has greatly improved for planar vapor adsorption. The sensitivity is mainly determined by the receptor sites on the QCM sensor. Since the chemical components of the imprinted polymers and the control polymer are almost identical, the observed selectivity suggests that some specific sites for the molecular recognition are formed on the cross-linked structure as expected. We can make the assumption that the cavities formed using hydroquinone and phenol as the templates show selective binding of planar aromatic compounds. Another hypothesis is that the template molecule creates a higher surface area for adsorption of molecules in the imprinted polymer resulting in increased partition coefficients compared to control polymers. If we assume that $\mathrm{HEP}$ and $\mathrm{CCl}_{4}$ do not bind selectively in imprinted sites, then there is some evidence for increased surface area in Table 1.6 since normalized partition coefficients for control polymers are always larger than 1 .

Since the acrylonitrile/acrylic acid polymers are in a membrane configuration, they can only provide limited surface areas compared to the acrylic copolymer powders. As a result, MIMs show much lower partition coefficients and do not appear to offer significant by improved sensitivity over PIB films. However, the existence of 
an imprinting effect is clearly apparent. As shown in Table $1.6 \mathrm{c}$, there is a significant variation in the selectivity of the imprinted films relative to the control polymer. The polymer imprinted with hydroquinone presents higher normalized partition coefficients toward TOL and BEZ than toward $\mathrm{CCl}_{4}$. This method may provide the possible advantage of being able to control the thickness of a sensing film and it may be possible to design the sensing layer to be specific for a certain targeted analyte in aqueous analytes. 
Table 1.5 Apparent partition coefficients $(\mathrm{K})^{*}$ of PIB

\begin{tabular}{c|c|c|c|c|c}
\hline PIB coating & TCE & TOL & BEZ & HEP & $\mathbf{C C l}_{\mathbf{4}}$ \\
\hline $\mathbf{2 . 2} \mathbf{~ k ~ H z}$ & 3100 & 2660 & 1830 & 1020 & 660 \\
$\mathbf{4 . 2} \mathbf{~ k H z}$ & 2950 & 2320 & 1500 & 1160 & 760 \\
$\mathbf{6 . 5} \mathbf{~ k H z}$ & 2860 & 2180 & 1400 & 1250 & 730 \\
$\mathbf{A v e r a g e}$ & $3000 \pm 99$ & $2400 \pm 202$ & $1600 \pm 184$ & $1140 \pm 95$ & $720 \pm 42$ \\
\hline
\end{tabular}

* $\mathrm{K}$ values were obtained base on a single point calibration.

Vapor concentrations : $300 \mathrm{ppm}$.

Table 1.6 Normalized partition coefficients of the MIPs with respect to PIB (K values from Figure 1.32 1.34.RSD for the normalized $k$ values is approximately $10 \%$. )

(a) MIPs: Methacrylic acid copolymers.

\begin{tabular}{|c|c|c|c|c|c|}
\hline \multirow{2}{*}{ MIP } & \multicolumn{5}{|c|}{ Vapors } \\
\hline & TCE & TOL & BEZ & HEP & $\mathrm{CCl}_{4}$ \\
\hline $\mathrm{BIP}(\mathrm{HQ})$ & 3.1 & 6.7 & 6.0 & 1.4 & 1.8 \\
\hline$B I P(P)$ & 2.1 & 4.0 & 2.4 & 1.0 & 1.5 \\
\hline $\mathrm{BIP}(\mathrm{C})$ & 1.7 & 1.5 & 1.3 & 1.1 & 1.1 \\
\hline IMP(HQ) & 1.9 & 4.1 & 1.8 & 1.6 & 1.2 \\
\hline $\operatorname{IMP}(P)$ & 1.4 & 2.3 & 1.5 & 0.8 & 0.8 \\
\hline $\operatorname{IMP}(\mathrm{C})$ & 1.2 & 1.0 & 0.4 & 0.4 & 0.8 \\
\hline
\end{tabular}


(b) MIPs: Acrylic acid copolymers

\begin{tabular}{c|ccccc}
\hline \multirow{2}{*}{ MIP } & \multicolumn{5}{|c}{ Vapors } \\
\cline { 2 - 6 } & TCE & TOL & BEZ & HEP & $\mathbf{C C l}_{4}$ \\
\hline BIP(HQ) & 3.9 & 18.0 & 11.4 & 3.1 & 2.1 \\
BIP(P) & 3.3 & 8.4 & 10.1 & 2.8 & 2.1 \\
BIP(C) & 2.8 & 2.5 & 2.2 & 2.9 & 2.9 \\
\hline IMP(HQ) & 3.1 & 6.7 & 6.0 & 1.4 & 1.5 \\
IMP(P) & 2.1 & 4.0 & 2.3 & 1.0 & 1.1 \\
IMP(C) & 1.7 & 1.5 & 1.3 & 1.0 & 2.5 \\
\hline
\end{tabular}

(c) MIPs: Acrylonitrile copolymers

\begin{tabular}{c|cccc}
\hline \multirow{2}{*}{ MIP } & \multicolumn{4}{|c}{ Vapors } \\
\cline { 2 - 5 } & TCE & TOL & BEZ & CCl $_{4}$ \\
\hline MIM(HQ) & 0.45 & 0.87 & 0.74 & 0.34 \\
MIM(P) & 0.23 & 0.47 & 0.44 & 0.35 \\
$\operatorname{MIM(C)}$ & 0.19 & 0.18 & 0.15 & 0.16 \\
\hline
\end{tabular}




\subsubsection{Comparisons of BIP and IMP}

The imprinted polymer particles obtained by grinding the polymer block (BIPs) are irregular in shape and size. When a cross-linking polymerization is started from a dilute, homogeneous monomer solution, spherical micro-gels with narrow size distribution (IMPs) are formed [24]. The latter particles might be expected to be superior for mechanical coupling to a QCM surface. For comparison, BIP and IMP particles are prepared using the same monomer components with identical molar ratios of monomer, cross-linker and template. In general, the BIPs exhibit higher apparent partition coefficients than the IMPs. Both imprinted polymers exhibit similar frequency-time response profiles toward vapor pulses (Figure 1.36). Time constants for adsorption and desorption on IMPs are similar to those in Table 1.4. The smaller imprinting effect on IMPs might be attributed to the synthesis conditions. The lower concentration of the monomer, cross-linker and template in a quite polar solvent (acetonitrile) may interfere with hydrogen-bonding interactions between the functional monomer and the template. Hence, imprinting is less efficient and binding capacity for the imprinted microspheres is lower than that of the block polymer. However, the enhanced partition coefficients for imprinted microspheres relative to the control microspheres is still evident (Table 1.5). 


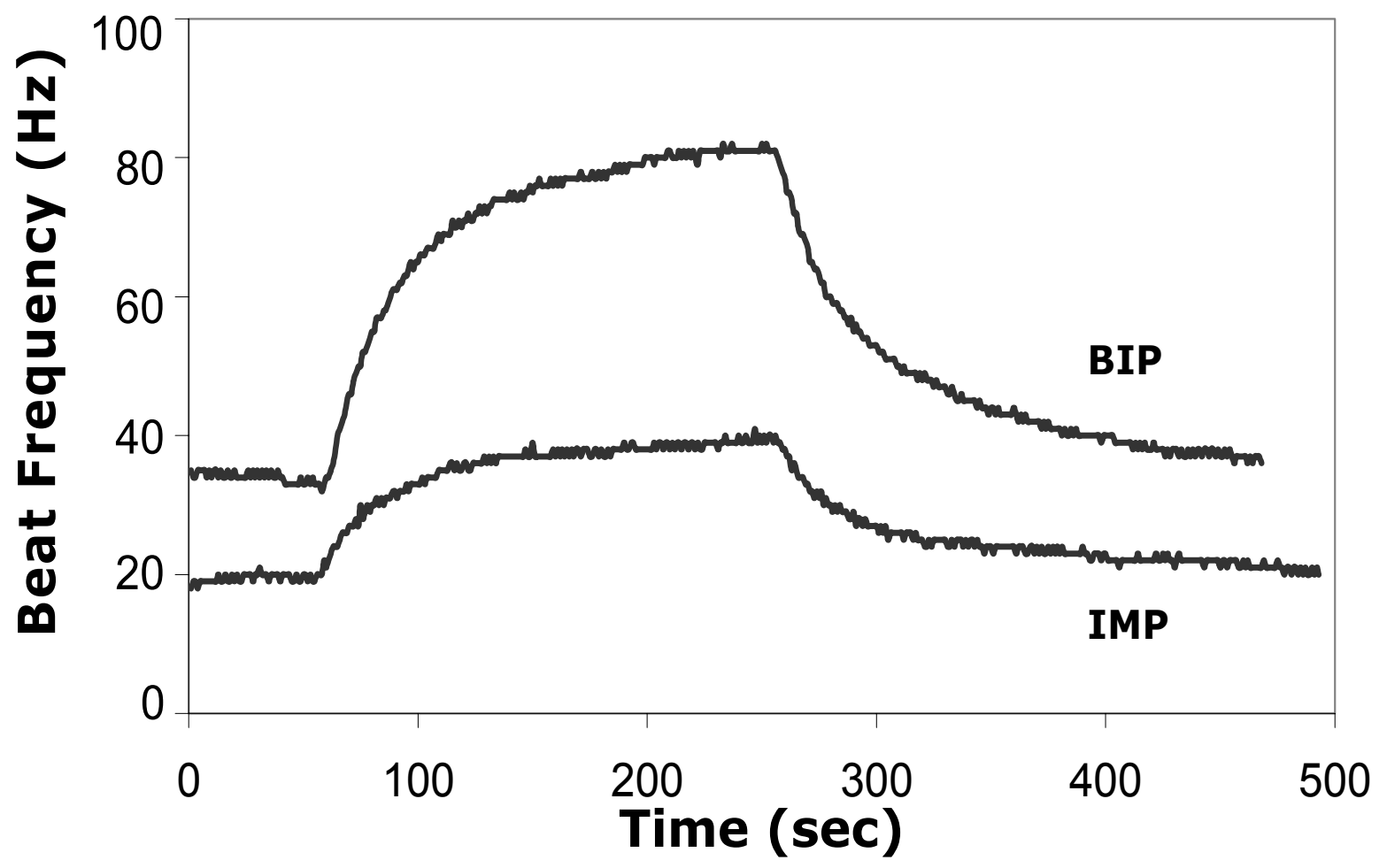

Figure 1.36 Typical real-time response plots of BIP and IMP to TOL vapors.

TOL vapor concentration: 300 ppm; polymer layer: 2 kHz PIB plus 2 kHz MIP coating; MIP: methacrylic acid copolymers imprinted with hydroquinone. Rise time constants: $(B I P)=14$ sec; $($ IMP $)=15 \mathrm{sec}$. Decay time constants: $(\mathrm{BIP})=43 \mathrm{sec} ;(\mathrm{IMP})=36 \mathrm{sec}$. 


\subsubsection{Influence of template concentration on the MIP performance}

In the imprinted polymer film, the density of templated sites should be determined by the relative amount of template molecule to the monomer and the cross-linker. QCM sensors coated with different MIP films prepared with different template concentrations are tested. Figure 1.37 shows the influence of the amount of hydroquinone used during the polymer preparation on binding performance of the resulting bulk imprinted polymer. As the mole ratio of hydroquinone to methacrylic monomer increases $(1: 1,2: 1 \& 3: 1)$, the apparent partition coefficients toward toluene and benzene increase by roughly $100 \%$. For non-planar heptanes, the partition coefficient changes with template mole ratio is less significant; the apparent $\mathrm{K}$ values are around 1,800 for all three imprinted polymers. Partition coefficients for trichloroethylene and carbon tetrachloride both increase by $70 \%$. These observations are consistent with a molecular imprinting mechanism combined possibly with an increased surface area as the template mole ratio increases. The template mole ratio cannot be increased much higher because of solubility limitations of hydroquinone in DMSO. Also, the higher template concentration of hydroquinone and phenol in the monomer mixture may inhibit the polymerization. 


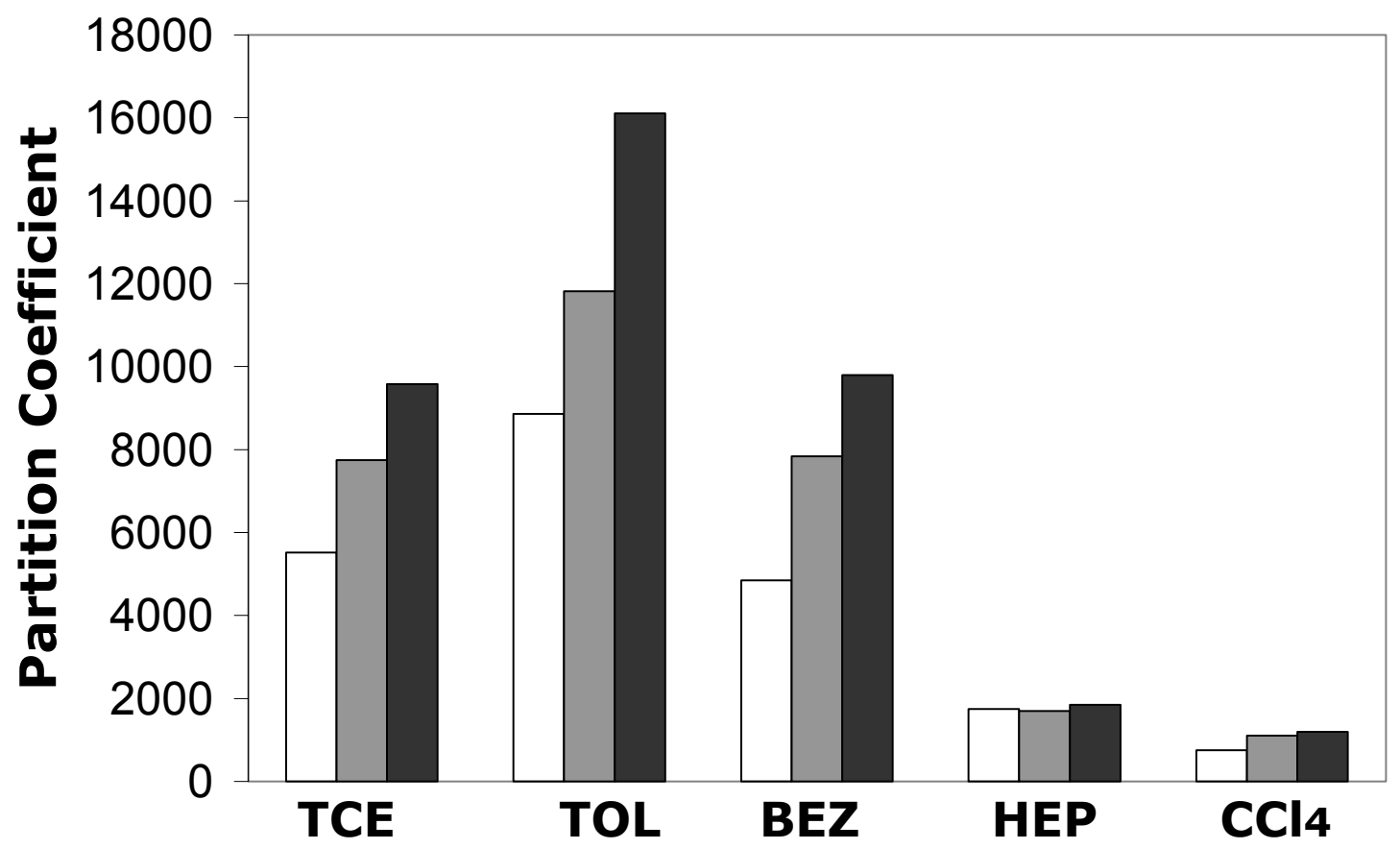

Figure 1.37 Bar figure of apparent partition coefficients comparison of BIP(HQ) with different template concentration. (polymer coating: $2 \mathrm{KHz}$ PIB plus 2KHz MIP). Template /monomer concentration mole ratio (hollow bar: 1:1; gray bar: 2:1; black bar: $₫:$ 3:1). The partition coefficients are averages from 3 trials, RSDs for 3 trials are ranged from $2.2 \sim 4.2 \%$. 


\subsubsection{Cross-linker effect}

Since the cross-linking monomer, EGDMA, is the main component for the polymers, appropriate levels of cross-linking are important in maintaining binding specificity of an imprinted polymer. The effect of cross-linker concentrations in MIP was investigated by varying the amount of cross-linker employed in the polymerization mixture (Figure 1.38). Cross-linker concentrations are varied from 0.5 to $10 \mathrm{mmol}$ (weight percent from 13 to $75 \%$ ). The corresponding control polymer is also synthesized to compare the relative selectivity of MIPs. When the levels of crosslinking are too low, the selectivity of the imprinted polymer decreases greatly. We hypothesize that the increased flexibility of the polymer results in the relaxation of the imprinted sites so that binding is less selective. The copolymer with higher proportions of cross-linker is rigid, leading to excellent selectivity. However, selectivity of polymers obtained at very high cross-linker concentrations is reduced. We hypothesize that the diffusion of substrate into the polymer matrix may be hindered. 


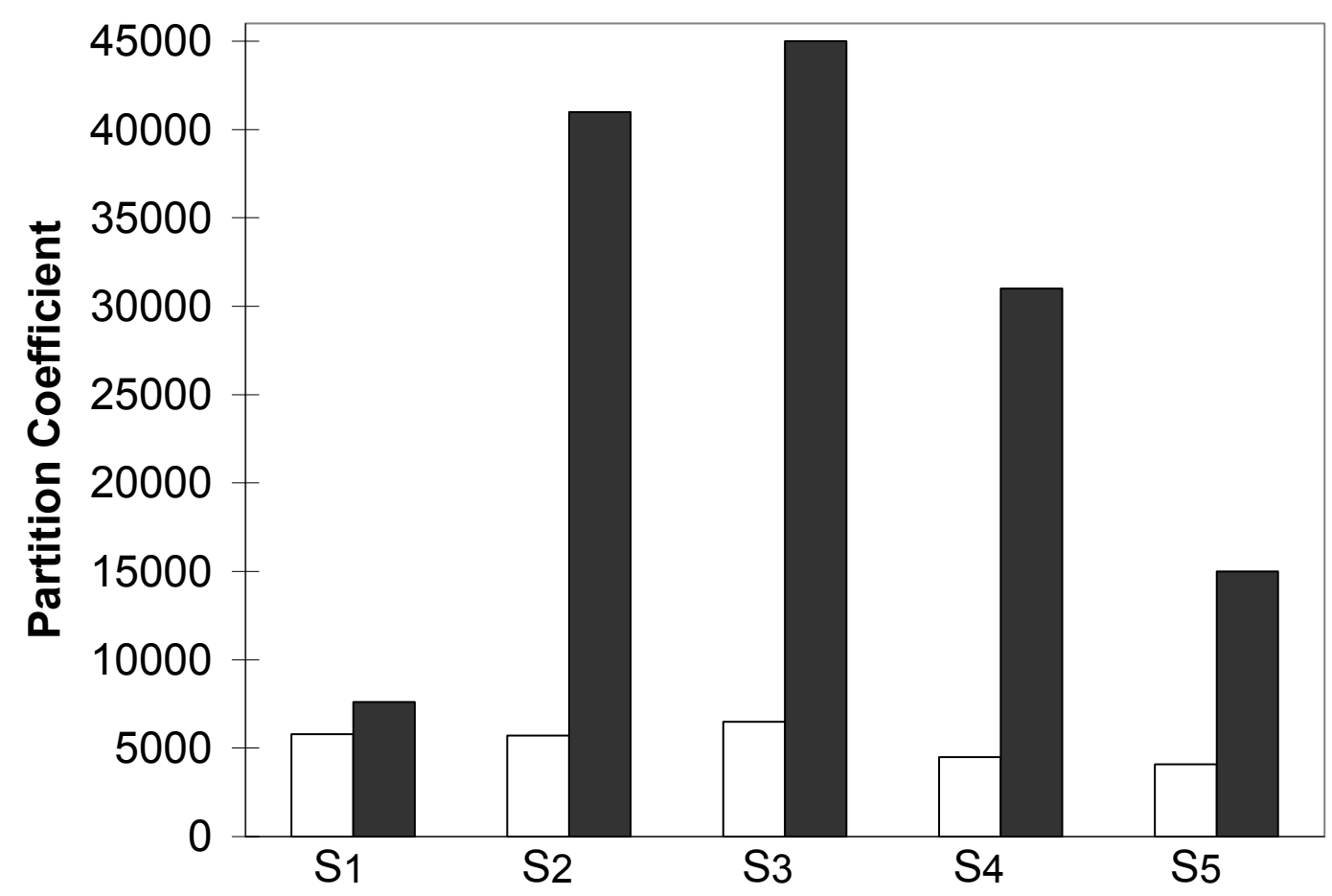

Figure 1.38 Cross-linker effect on sensitivity.

Black bar: Acrylic copolymer imprinted with $\mathrm{HQ}(\mathrm{BIP}(\mathrm{HQ}))$; hollow bar: Control polymer. TOL vapor Concentration: 300 ppm.

$S_{1}: 0.5 \mathrm{mmol}$ EGDMA (13\%); $S_{2}: 2 \mathrm{mmol}$ EGDMA(36\%); $S_{3}: 3 \mathrm{mmol}$ EGDMA

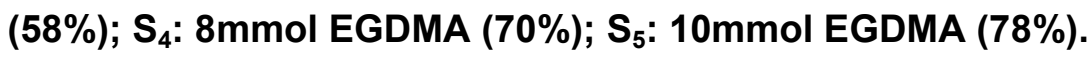

The partition coefficients are averages from 3 trials; RSDs for 3 trials are $1.4 \sim 3.7 \%$ 


\subsubsection{Lifetime and Reproducibility}

Molecularly imprinted polymers synthesized with the same recipe but in different batches are also evaluated with respect to preparation variations. The partition coefficients of all three sets of polymers were obtained by QCM experiments and compared. The data are summarized in Table 1.7 a. The normalized partition coefficients with respect to PIB toward different vapors are listed in Table $1.7 \mathrm{~b}$. The imprinted polymers from different batches exhibit relatively large deviations toward TOL vapor ( $17 \sim 18 \%$ RSD); the partition coefficient data for the control polymers is more reproducible (about 7\% for RSD). However, the obtained normalized partition coefficients show the similar selective pattern as described previously. The agreement suggests that our methodology for MIP synthesis is successful.

The stability of the MIP coated QCM is studied by repeating measurements of a fixed concentration of toluene vapor over two weeks( Figure 1.39). It is found that the sensor stored in the air at room temperature retains about $85 \%$ of its original sensitivity after two weeks. The coating mass of the QCM, as indicated by the baseline frequency, also decreases by $10 \%$ over two weeks. The decrease of sensitivity is mostly likely due to the loss of coating material during storage. 
Table 1.7 (a) Reproducibility comparison of MIPs from different batches*.

\begin{tabular}{c|c|c|c|c}
\hline & $\mathbf{1}$ & $\mathbf{2}$ & $\mathbf{3}$ & $\mathbf{R S D}(\%)$ \\
\hline BIP (HQ) & 43000 & 33000 & 52000 & 18 \\
BIP(P) & 20000 & 18000 & 27000 & 17 \\
CP & 6000 & 5300 & 6200 & 6.6 \\
\hline
\end{tabular}

* Test vapor : 300 ppm TOL; MIP component: Acrylic acid imprinted polymer.

(b) Normalized partition coefficients of MIPs from different batches with respect to PIB*

\begin{tabular}{c|c|c|c|c|c}
\hline & TCE & TOL & BEZ & HEP & $\mathbf{C C l}_{\mathbf{4}}$ \\
\hline BIP (HQ) I & 3.9 & 18.0 & 11.4 & 3.1 & 2.1 \\
BIP (HQ) II & 3.0 & 13.8 & 8.8 & 2.4 & 1.8 \\
BIP (HQ) III & 4.4 & 21.6 & 12.6 & 3.6 & 2.4 \\
RSD(\%) & 15.7 & 17.9 & 14.5 & 16.2 & 11.6 \\
\hline
\end{tabular}

* Test vapor : 300 ppm TOL; MIP component: Acrylic acid imprinted polymer. The $K$ values are averages based on 3 trials. 


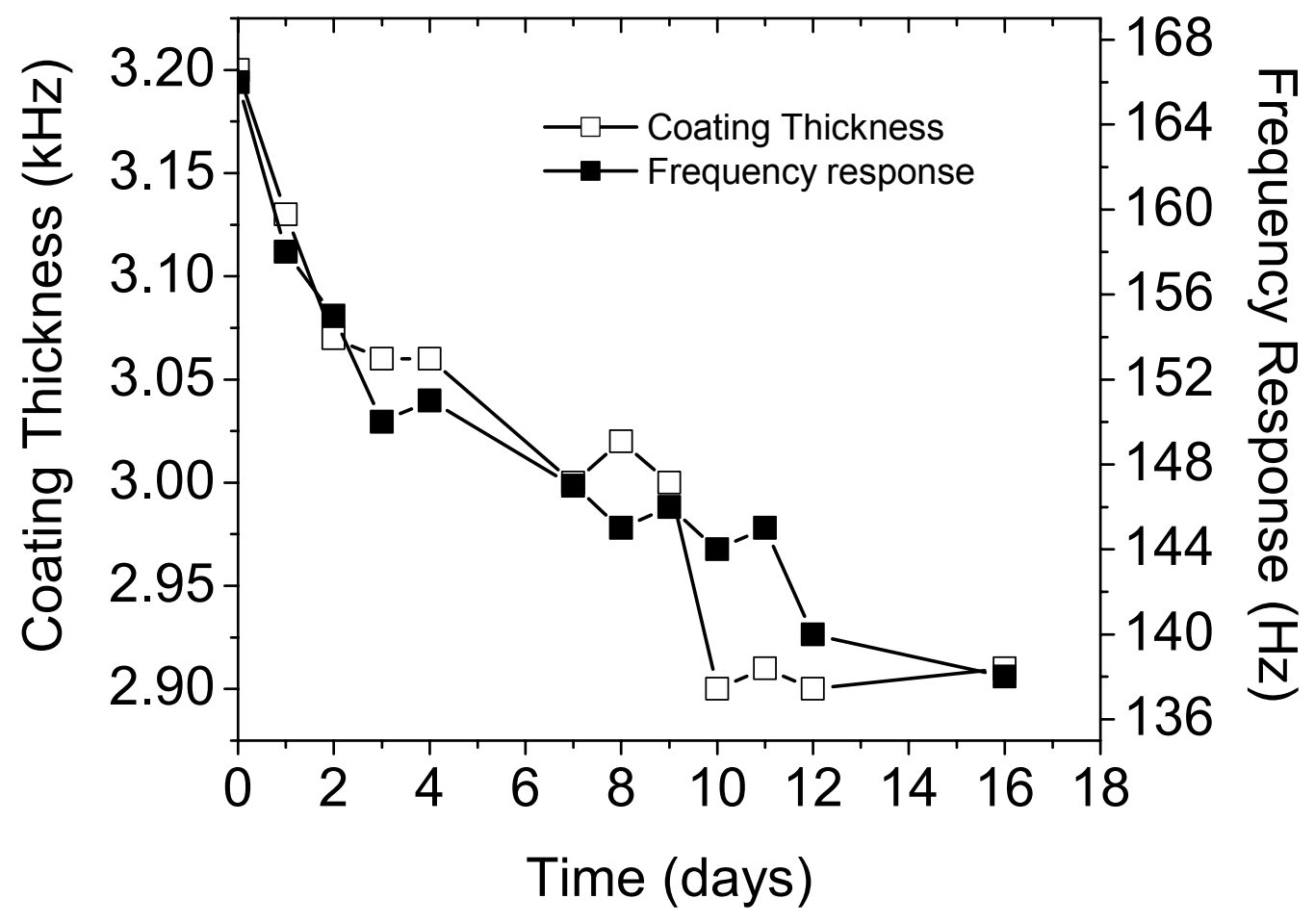

Figure 1.39 Stability of QCM coated with BIP(HQ), Test vapor : 300 ppm TOL; MIP component: Acrylic acid imprinted polymer. 


\subsection{Vapor Sorption by Dry powder}

The MIP adsorbents can adsorb vapors via a range of non-specific interactions in addition to the desired 'template' associated specific adsorption. Non-specific adsorption sites occur on the polymer surface, in the polymer matrix, and next to imprinted cavities. The maximum vapor sorption capacities of powder samples of the MIPs can be determined from weight changes after extended exposures to air saturated with the respective vapors.

This experiment is done by measuring the total weight increase for the bulk powder. These powders are also tested to determine the reversible desorption of the vapor under vacuum. The commercial adsorbents, Tenax TA ${ }^{\circledR}$, Tenax GR ${ }^{\circledR}$ and synthesized molecularly imprinted polymers are compared. Tenax TA and GR are selected as reference compounds because they are widely used for volatile organic compounds (VOCs) sampling. The vapors include benzene (BEZ), toluene (TOL), trichloroethylene (TCE), cyclohexane, heptanes and dichloromethane. The experimental mass percent changes for each organic vapor are shown in Table 1.8.

The results show that benzene and toluene vapors cause higher mass changes in the imprinted polymers than the non-imprinted polymers, Tenax TA and Tenax GR, which is consistent with the partition coefficient results, indicating some selectivity based on imprinting. For the cyclohexane and heptane vapors, all the tested adsorbents show the mass increase in the range of $10 \sim 25 \%$; no obvious difference is observed. All polymer powders exhibit greater affinity to TCE and dichloromethane, especially for the latter compound. The control polymer powders and the Tenax adsorbents became slightly "wet" or "soaked" after 24 hours exposure with 
dichloromethane. Consequently, no conclusion can be drawn with respect to the adsorption of dichloromethane. For cyclohexane and heptanes, the adsorption is anticipated to be non-selective. The results suggest that the adsorption surface area of all the polymer are roughly the same. Therefore, the enhanced adsorption of toluene in the block polymer imprinted with hydroquinone provides additional support for the existence of imprinted sites selective for toluene. 
Table 1.8 Vapor Uptake by Various Adsorbents ${ }^{\dagger}$.

a. Toluene vapor adsorption

\begin{tabular}{c|c|c|c}
\hline Adsorbent & $\begin{array}{c}\text { Sample Weight } \\
\mathbf{( g )}\end{array}$ & $\begin{array}{c}\text { Weight Change } \\
\mathbf{( g )}\end{array}$ & $\begin{array}{c}\text { Percent Change } \\
\mathbf{( \% )}\end{array}$ \\
\hline Tenax TA & 0.0506 & 0.0121 & 24 \\
Tenax GR & 0.0611 & 0.0123 & 20 \\
IMP (HQ) & 0.0546 & 0.0147 & 27 \\
IMP(P) & 0.0581 & 0.0137 & 24 \\
IMP (CP) & 0.0572 & 0.072 & 13 \\
BIP (HQ) & 0.0521 & 0.0338 & 65 \\
BIP ( P) & 0.0635 & 0.041 & 65 \\
BIP (CP) & 0.0621 & 0.0157 & 25 \\
\hline
\end{tabular}

b. Benzene vapor adsorption

\begin{tabular}{c|c|c|c}
\hline Adsorbent & $\begin{array}{c}\text { Sample Weight } \\
\mathbf{( g )}\end{array}$ & $\begin{array}{c}\text { Weight Change } \\
\mathbf{( g )}\end{array}$ & $\begin{array}{c}\text { Percent Change } \\
\mathbf{( \% )}\end{array}$ \\
\hline Tenax TA & 0.0451 & 0.0226 & 50 \\
Tenax GR & 0.0600 & 0.0212 & 35 \\
IMP (HQ) & 0.0505 & 0.0265 & 52 \\
IMP (P) & 0.0511 & 0.0289 & 57 \\
IMP (CP) & 0.0466 & 0.0095 & 20 \\
BIP (HQ) & 0.0543 & 0.0336 & 62 \\
BIP ( P) & 0.0687 & 0.044 & 64 \\
BIP (CP) & 0.0749 & 0.0366 & 49 \\
\hline
\end{tabular}

\footnotetext{
${ }^{\dagger}$ The data are the averages of weight changes from two successive adsorption trials.
} 
c. Cyclohexane vapor adsorption

\begin{tabular}{c|c|c|c}
\hline Adsorbent & $\begin{array}{c}\text { Sample Weight } \\
\mathbf{( g )}\end{array}$ & $\begin{array}{c}\text { Weight Change } \\
\mathbf{( g )}\end{array}$ & $\begin{array}{c}\text { Percent Change } \\
\mathbf{( \% )}\end{array}$ \\
\hline Tenax TA & 0.0602 & 0.0114 & 19 \\
Tenax GR & 0.0520 & 0.0082 & 16 \\
IMP (HQ) & 0.0466 & 0.0082 & 18 \\
IMP (P) & 0.0753 & 0.0125 & 17 \\
IMP (CP) & 0.0568 & 0.0119 & 21 \\
BIP (HQ) & 0.0644 & 0.0165 & 26 \\
BIP ( P) & 0.0697 & 0.0153 & 22 \\
BIP (CP) & 0.0701 & 0.0094 & 14 \\
\hline
\end{tabular}

d. Heptane vapor adsorption

\begin{tabular}{c|c|c|c}
\hline Adsorbent & $\begin{array}{c}\text { Sample Weight } \\
\mathbf{( g )}\end{array}$ & $\begin{array}{c}\text { Weight Change } \\
\mathbf{( g )}\end{array}$ & $\begin{array}{c}\text { Percent Change } \\
\mathbf{( \% )}\end{array}$ \\
\hline Tenax TA & 0.0467 & 0.0099 & 21 \\
Tenax GR & 0.0597 & 0.0095 & 16 \\
IMP (HQ) & 0.052 & 0.0073 & 14 \\
IMP (P) & 0.0539 & 0.0054 & 10 \\
IMP (CP) & 0.0553 & 0.0066 & 12 \\
BIP (HQ) & 0.0603 & 0.0082 & 14 \\
BIP ( P) & 0.050 & 0.0055 & 11 \\
BIP (CP) & 0.051 & 0.0078 & 15 \\
\hline
\end{tabular}


f. TCE vapor adsorption

\begin{tabular}{c|c|c|c}
\hline Adsorbent & $\begin{array}{c}\text { Sample Weight } \\
\mathbf{( g )}\end{array}$ & $\begin{array}{c}\text { Weight Change } \\
\mathbf{( g )}\end{array}$ & $\begin{array}{c}\text { Percent Change } \\
\mathbf{( \% )}\end{array}$ \\
\hline Tenax TA & 0.0588 & 0.0328 & 56 \\
Tenax GR & 0.0456 & 0.0251 & 55 \\
IMP (HQ) & 0.0349 & 0.0162 & 46 \\
IMP (P) & 0.0522 & 0.0219 & 42 \\
IMP (CP) & 0.0638 & 0.0295 & 46 \\
BIP (HQ) & 0.0637 & 0.0683 & $110^{*}$ \\
BIP ( P) & 0.0679 & 0.0826 & $120^{*}$ \\
BIP (CP) & 0.0659 & 0.0694 & $110^{*}$ \\
\hline
\end{tabular}

* Adsorbent became "wet" after exposure

g. Dichloromethane vapor adsorption

\begin{tabular}{c|c|c|c}
\hline Adsorbent & $\begin{array}{c}\text { Sample Weight } \\
\mathbf{( g )}\end{array}$ & $\begin{array}{c}\text { Weight Change } \\
\mathbf{( g )}\end{array}$ & $\begin{array}{c}\text { Percent Change } \\
\mathbf{( \% )}\end{array}$ \\
\hline Tenax TA & 0.0177 & 0.159 & $900^{*}$ \\
Tenax GR & 0.0771 & 0.1211 & 160 \\
IMP (HQ) & 0.0464 & 0.0532 & 110 \\
IMP (P) & 0.0420 & 0.049 & 120 \\
IMP (CP) & 0.0540 & 0.0979 & $180^{*}$ \\
BIP (HQ) & 0.0593 & 0.0551 & 93 \\
BIP ( P) & 0.0677 & 0.0654 & 97 \\
BIP (CP) & 0.0544 & 0.4315 & $790^{*}$ \\
\hline
\end{tabular}

* Adsorbent became 'wet” after exposure 


\subsection{Breakthrough Studies}

Equation 4 has two parameters, $W_{e}$, the adsorption capacity; and $K_{v}$, the rate constant for adsorption, that can be calculated from experimental data. The equation also predicts that the breakthrough time varies linearly with the weight of the adsorbent if $\mathrm{C}, \mathrm{C}_{0}$ and $\rho$ remains constant.

$$
t_{b}=\frac{W_{e}}{C_{0} Q}\left[W-\frac{\rho_{B} Q}{K_{v}} \ln \left(\frac{C_{0}-C}{C}\right)\right]
$$

Experimental values of the breakthrough time $t_{b}$ for each vapor and adsorbent system are obtained at several vapor concentrations as a functions of the adsorbent weight. In this experiment, the breakthrough time is defined as the time when the concentration of the vapor in the exit stream reaches $10 \%$ of the inlet concentration. Linear regression analyses of the breakthrough time versus the adsorbent weight are given in Table 1.9 and shown graphically in Figure 1.40 for toluene vapor.

Correlation coefficients $\left(\mathrm{R}^{2}\right)$ range from 0.9672 to 0.9991 .

Table 1.10 shows the calculated values of adsorption capacity $\left(W_{\mathrm{e}}\right)$ and the rate constant $\left(K_{\mathrm{v}}\right)$ derived from the regression equations. A sample calculation for $W_{\mathrm{e}}$ and $K_{\mathrm{v}}$ is shown below. 


$$
\begin{aligned}
W_{e}(m g / g)=C_{0} Q b & =(3.762 * 140) * 20 *(194) / 10^{6} \\
& =2.04(\mathrm{mg} / \mathrm{g})
\end{aligned}
$$

Given flow rate $Q: 20 \mathrm{ml} / \mathrm{min}$; slope $b: 194 \mathrm{~min} / \mathrm{g} ; 140 \mathrm{ppm}$ toluene vapor: $(3.762 * 140) \mathrm{mg} / \mathrm{m}^{3}$;

$$
\begin{aligned}
K_{v}(1 / \mathrm{min}) & =-W_{e} * \rho^{*} \operatorname{Ln}\left(\frac{C_{0}-C}{C}\right) /\left(C_{0} * a\right) \\
& =-2.04 * 10^{6} *(2.197) /(140 * 3.762) *(-0.724) \\
& =11797(1 / \mathrm{min})
\end{aligned}
$$

Assuming polymer density $\rho: 1 \mathrm{~g} / \mathrm{cm}^{3}$;

Given $\operatorname{Ln}\left(\frac{C_{0}-C}{C}\right)=\operatorname{Ln}\left(\frac{C_{0}-0.1 C_{0}}{0.1 C_{0}}\right)=\operatorname{Ln} 9=2.197$

140 ppm toluene vapor: $(3.762 * 140) \mathrm{mg} / \mathrm{m}^{3}$; intercept $b:-0.724$.

Tenax adsorbents are macro-porous polymers manufactured from diphenyl-pphenylene oxide (DPPO). The $W_{\mathrm{e}}$ data of Tenax TA and GR from this experiment are in agreement with the reported values in literatures [43]. Since Tenax TA has a relatively higher surface area compared with Tenax GR, the Tenax TA exhibits relatively larger adsorption capacities toward the tested vapors. However, the adsorption capacities of Tenax TA for TOL and BEZ are much smaller than those of the imprinted polymers. It is obviously clear that BIP(HQ) polymer possess the highest adsorption capacities toward TOL, BEZ and HEP. The BIP control polymer exhibits relatively smaller $W_{e}$ values toward toluene and benzene vapors when compared with the corresponding imprinted polymers. A low adsorption capacity of imprinted microsphere polymers (IMP) is observed for heptanes adsorption, and $W_{e}$ is 
close to the value of Tenax GR. Conversely, BIP polymers still exhibit rather high $W_{\mathrm{e}}$ values for HEP, which is comparable to the $W_{e}$ values for Tenax TA. An assumption we can make here is that larger surface area may contribute to the adsorption capacity. The imprinting process not only can form some specific site for selective binding, but also create a large surface area. As a result, the imprinted BIP polymer always shows higher adsorption capacity than its corresponding control polymer. The results from breakthrough study also imply that BIP polymers present larger surface area than IMPs.

In order to verify the accuracy of $W_{\mathrm{e}}$ values, the vapor adsorption on Tenax polymers and imprinted polymer adsorbents is studied at different flow rates ( 20 $\mathrm{ml} / \mathrm{min}, 30 \mathrm{ml} / \mathrm{min}$ and $50 \mathrm{ml} / \mathrm{min}$ ). Duplicate experiments are in very good agreement with relative standard deviation of $1.1 \%$ for linear regression slopes. Presented in Figure 1.41 are plots of the 10\% breakthrough time versus TOL for three different flow rates. As the theory predicts, all the plots in Figure 1.41 are parallel, and, as the flow rate increases, $\left(\mathrm{C}_{0} \mathrm{Q}\right.$ is kept constant), the value of the intercept decreases. The rate constants $\mathrm{K}_{\mathrm{v}}$ increases as the flow rate increases.

The adsorption capacities are plotted against the concentrations in Figure 1.42. The plots of $W_{e}$ vs $\mathrm{C}_{0}$ are the adsorption isotherm of toluene for Tenax GR and BIP (HQ). The BIP isotherm exhibits the greater slope and the higher adsorption capacity. The slope of the isotherm can be converted to a partition coefficient of about 20,000, which is in good agreement with partition coefficients obtained from QCM studies. On the other hand, no specific trend is noted for the rate constant, $\mathrm{K}_{\mathrm{v}}$. This kinetic parameter may differ with the various methods and flow rates. 
Table 1.9 Results of breakthrough experiments $\left(t_{b}=a+b W_{e}\right)^{*}$

a. $140 \mathrm{ppm}$ Toluene, flow rate: $20 \mathrm{ml} / \mathrm{min}$

\begin{tabular}{c|c|c|c}
\hline Adsorbent & $\mathrm{a}(\mathrm{min})$ & $\mathrm{b}(\mathrm{min} / \mathrm{g})$ & Correlation \\
& & & coefficient \\
\hline Tenax GR & -0.724 & 194 & 0.989 \\
Tenax TA & -4.82 & 672 & 0.996 \\
MIP(HQ) & -15.2 & 1420 & 0.978 \\
MIP (P) & -6.86 & 1010 & 0.977 \\
MIP (CP) & -4.46 & 573 & 0.984 \\
IMP(HQ) & -9.30 & 1100 & 0.994 \\
IMP (P) & -5.95 & 806 & 0.993 \\
IMP(CP) & -1.23 & 207 & 0.999 \\
\hline
\end{tabular}

b. 440 ppm HEP, flow rate: $20 \mathrm{ml} / \mathrm{min}$;

\begin{tabular}{c|c|c|c}
\hline Adsorbent & $\mathrm{a}(\mathrm{min})$ & $\mathrm{b}(\mathrm{min} / \mathrm{g})$ & Correlation \\
& & & coefficient \\
\hline Tenax GR & -0.0680 & 17.7 & 0.990 \\
Tenax TA & -0.502 & 85.0 & 0.994 \\
MIP(HQ) & -0.648 & 97.5 & 0.997 \\
MIP (P) & -0.242 & 73.7 & 0.994 \\
MIP (CP) & -0.343 & 48.9 & 0.999 \\
IMP(HQ) & -0.106 & 27.2 & 0.967 \\
IMP (P) & -0.0663 & 20.8 & 0.971 \\
IMP(CP) & -0.0858 & 29.0 & 0.988 \\
\hline
\end{tabular}

${ }^{*}$ Linear regression yields slope, intercept. The breakthrough times plotted are the averages of two trials. 
c. 370 ppm Benzene, flow rate: $20 \mathrm{ml} / \mathrm{min}$

\begin{tabular}{c|c|c|c}
\hline Adsorbent & $\mathrm{a}(\mathrm{min})$ & $\mathrm{b}(\mathrm{min} / \mathrm{g})$ & Correlation \\
& & & coefficient \\
\hline Tenax GR & -0.148 & 82.9 & 0.979 \\
Tenax TA & -0.890 & 173 & 0.985 \\
MIP(HQ) & -3.57 & 554 & 0.977 \\
MIP (P) & -2.11 & 385 & 0.996 \\
MIP (CP) & -0.556 & 185 & 0.994 \\
IMP(HQ) & -3.43 & 463 & 0.998 \\
IMP (P) & -3.23 & 395 & 0.999 \\
IMP(CP) & -1.95 & 290 & 0.992 \\
\hline
\end{tabular}




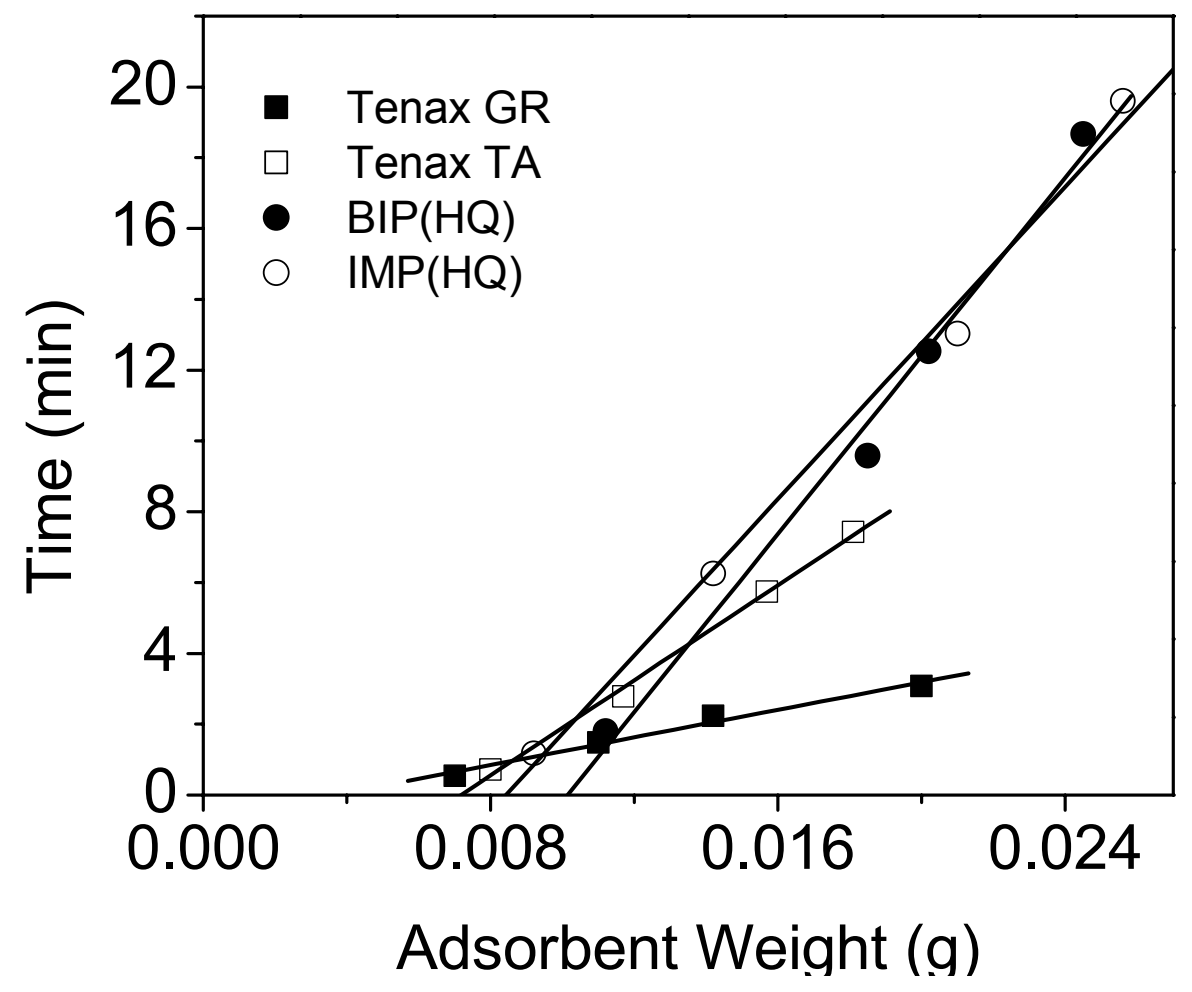

Figure 1.40 Linear regression plots of the breakthrough time as a function of adsorbent weight.

Toluene vapor concentration (140 ppm), flow rate: $20 \mathrm{ml} / \mathrm{min}$ 
Table 1.10 Adsorption parameters derived from regression equations

a. $140 \mathrm{ppm}$ TOL, flow rate: $20 \mathrm{ml} / \mathrm{min}$ (RSDs for We values range from $0.8 \sim 3.1 \%$ )

\begin{tabular}{l|l|l|l|l|l|l|l|l}
\hline Adsorbent & Tenax & Tenax & BIP & BIP & BIP & IMP & IMP & IMP \\
& GR & TA & (HQ) & (P) & (CP) & (HQ) & (P) & (CP) \\
\hline $\mathbf{W}_{\mathbf{e}}(\mathbf{m g} / \mathbf{g})$ & 2.04 & 7.07 & 14.9 & 10.6 & 6.03 & 11.6 & 8.49 & 2.18 \\
$\mathbf{K}_{\mathbf{v}}(\mathbf{1} / \mathbf{m i n})$ & 12000 & 6100 & 4100 & 6500 & 5600 & 5200 & 6000 & 7400 \\
\hline
\end{tabular}

b. 370 ppm BEZ, flow rate: $20 \mathrm{ml} / \mathrm{min}$ (RSDs for We values range from 1.7 2.8\%)

\begin{tabular}{l|l|l|l|l|l|l|l|l}
\hline Adsorbent & Tenax & Tenax & BIP & BIP & BIP & IMP & IMP & IMP \\
& GR & TA & $\mathbf{( H Q )}$ & $\mathbf{( P )}$ & $\mathbf{( C P )}$ & $\mathbf{( H Q )}$ & $\mathbf{( P )}$ & $\mathbf{( C P )}$ \\
\hline $\mathbf{W}_{\mathbf{e}}(\mathbf{m g} / \mathbf{g})$ & 1.96 & 4.09 & 13.1 & 9.08 & 4.36 & 10.9 & 9.31 & 6.83 \\
$\mathbf{K}_{\mathbf{v}}(\mathbf{1} / \mathbf{m i n})$ & 25000 & 8600 & 6810 & 8000 & 15000 & 5900 & 5400 & 55000 \\
\hline
\end{tabular}

c. $440 \mathrm{ppm}$ HEP, flow rate: $20 \mathrm{ml} / \mathrm{min}$ (RSDs for We values range from $2.0 \sim 4.2 \%$ )

\begin{tabular}{l|l|l|l|l|l|l|l|l}
\hline Adsorbent & Tenax & Tenax & BIP & BIP & BIP & IMP & IMP & IMP \\
& GR & TA & $\mathbf{( H Q )}$ & $\mathbf{( P )}$ & $\mathbf{( C P )}$ & $\mathbf{( H Q )}$ & $\mathbf{( P )}$ & $\mathbf{( C P )}$ \\
\hline $\mathbf{W}_{\mathbf{e}}(\mathbf{m g} / \mathbf{g})$ & 0.64 & 3.05 & 3.50 & 2.64 & 1.75 & 0.97 & 0.75 & 1.04 \\
$\mathbf{K}_{\mathrm{v}}(\mathbf{1} / \mathbf{m i n})$ & 11000 & 7500 & 6600 & 13000 & 6300 & 11000 & 13000 & 15000 \\
\hline
\end{tabular}




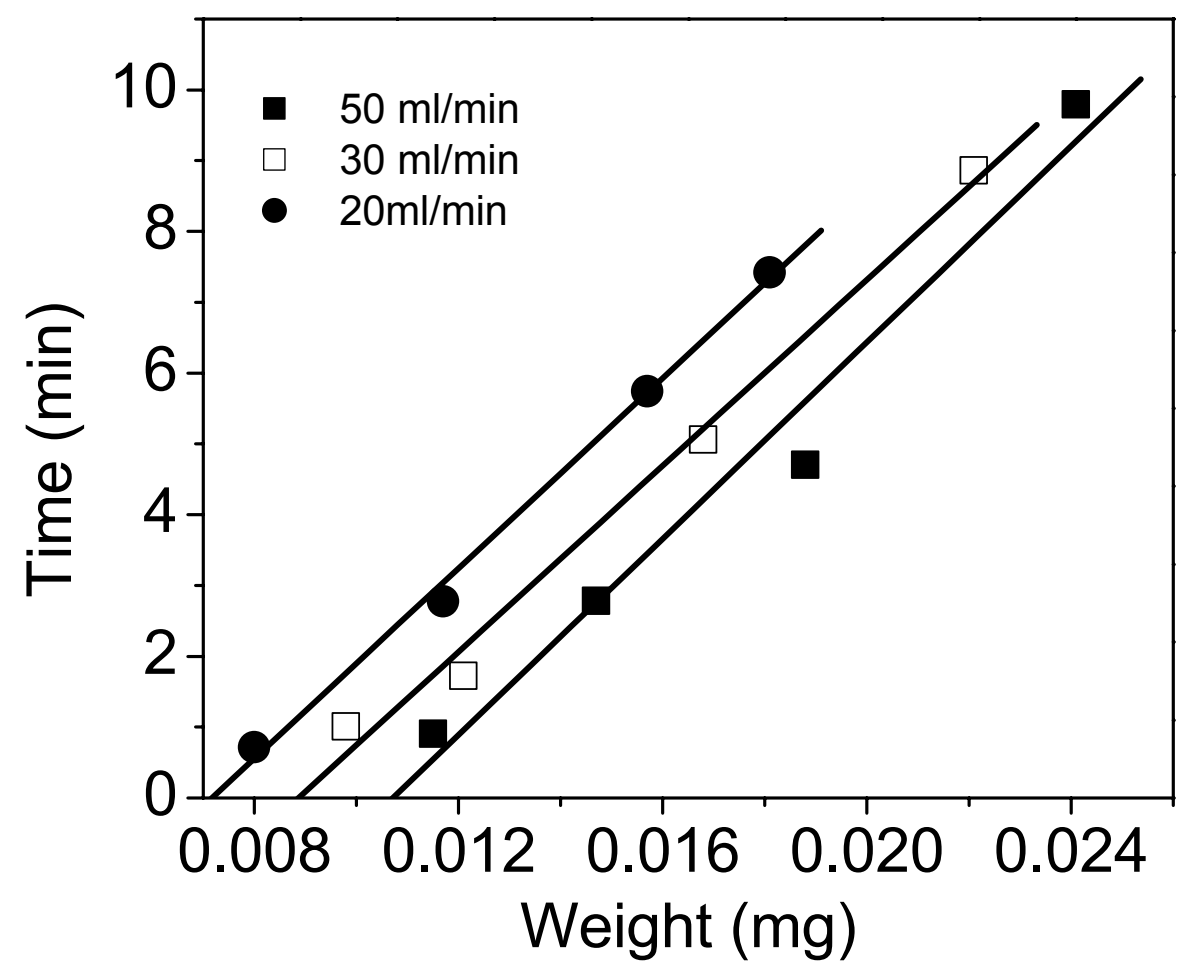

Figure 1.41 Adsorbent capacity as a function of vapor flow rates.

$\left(C_{0}{ }^{*} Q\right.$ is kept constant during the tests)

$20 \mathrm{ml} / \mathrm{min}: t_{b}=-4.82+672 W, \mathrm{R}^{2}=0.996, W_{\mathrm{e}}=7.1 \mathrm{mg} / \mathrm{g}$;

$30 \mathrm{ml} / \mathrm{min}: t_{b}=-5.83+657 W, R^{2}=0.990, W_{\mathrm{e}}=6.9 \mathrm{mg} / \mathrm{g}$;

$50 \mathrm{ml} / \mathrm{min}: t_{b}=-7.43+693 W, \mathrm{R}^{2}=0.972, W_{\mathrm{e}}=7.3 \mathrm{mg} / \mathrm{g}$.

RSD for the regression slopes: $1.1 \%$; RSD for the $W_{\mathrm{e}}: \mathbf{2 . 3} \%$ 


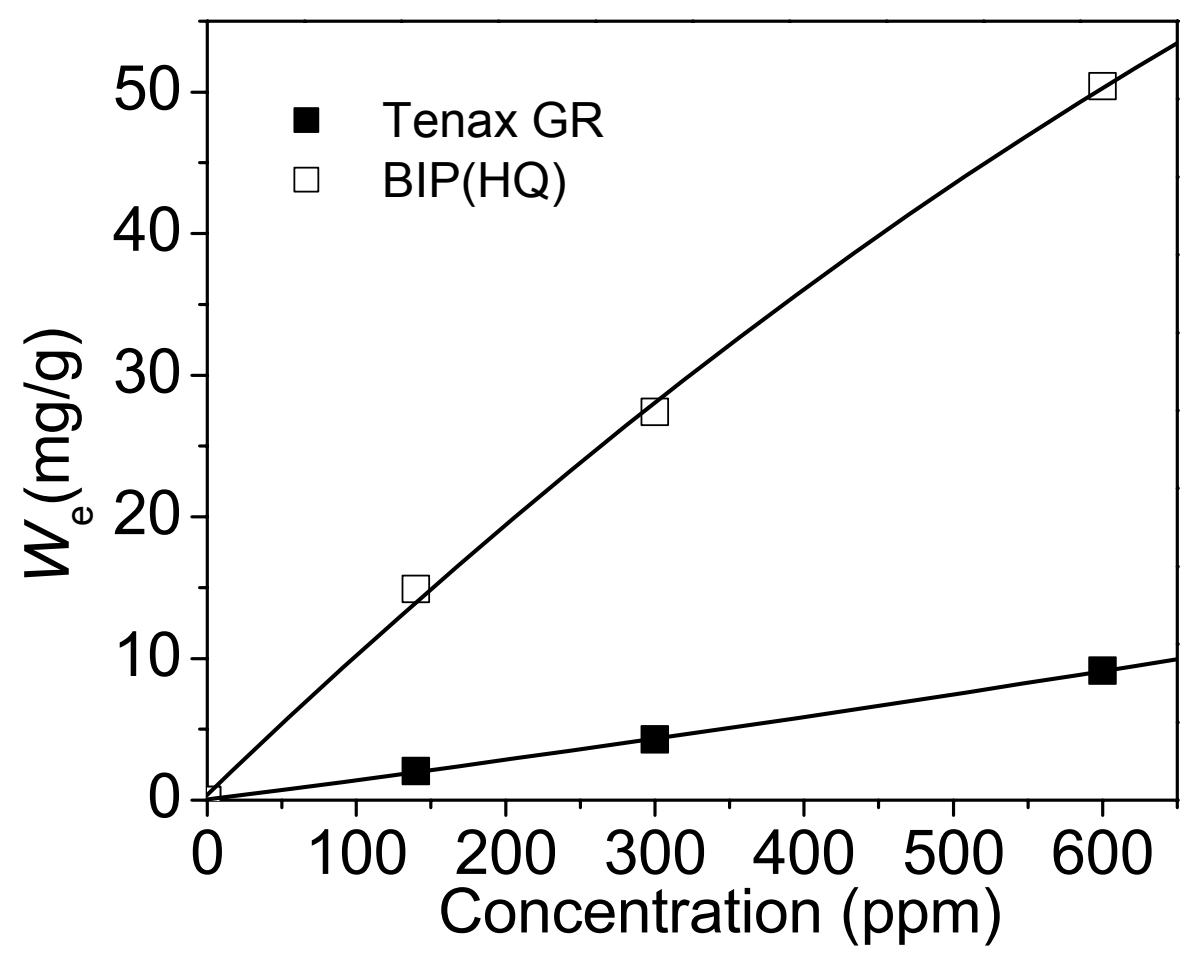

Figure 1.42 Adsorption isotherms for BIP (HQ) and Tenax GR.

Test Vapor: TOL, flow rate: $20 \mathrm{ml} / \mathrm{min}$. Data resulted from one trial. 


\section{Conclusion}

To our knowledge, enhanced adsorption of analytes from the gas phase into molecularly imprinted sites in a rigid polymer has not been unambiguously demonstrated until this study. In this study, we have selected molecules that should have relatively weak interactions with any adsorbent, since they are nonpolar and cannot form hydrogen bonds. Because there are no specific binding functional groups, the size and the shape of the cavities determine the strength of interactions and therefore the selectivity of the polymer. The imprinting focuses on shape (planar) and size (roughly the dimension of a benzene ring). The artificial molecular recognition system shows clearly a pronounced pattern of selectivity even with these small template molecules without functionality.

In this work, various molecularly imprinted polymers including block imprinted polymers (BIP), imprinted microsphere polymers (IMP) and molecularly imprinted membranes (MIM) and their corresponding control polymers have been synthesized and characterized by quartz crystal microbalances, vapor sorption measurements and breakthrough measurements. Organic vapors with different molecular shape and size are selected as the target analytes. Successful imprinting is supported by the following evidence.

(a) The imprinted polymers show greatly enhanced partition coefficients for the planar molecules toluene, benzene and TCE relative to the partition coefficients for the nonimprinted polymers. The time constant for desorption of planar toluene on an imprinted polymer is significantly higher than the desorption time constants of non-imprinted polymer or poly(isobutylene). MIMs show much lower partition 
coefficients and do not appear to offer significant improved sensitivity over PIB films. However, the existence of an imprinting effect is noticeable.

(b) After normalization of the partition coefficients with respect to the partition coefficients of PIB, it is clear that the imprinted polymers are selective for planar analytes over non-planar analytes, especially the block imprinted polymers (BIP).

(c) Experiment data from vapor sorbent measurements and breakthrough studies are in great agreement with the results concluded from QCM characterization. Imprinted polymers not only have higher adsorption capacities compared with the non-imprinted polymers, but offer an obvious advantage over the traditional adsorbents (Tenax polymers ) commonly used in vapor sampling. 


\section{References}

[1] Janata, J.; Jasowicz, M. Anal. Chem. 1998 (70), 179R-208 R.

[2] Cunningham, A. J. Introduction to bioanalytical sensors, New York : Wiley, c1998.

[3] Wulff, G. Angew, Chem. Int. Ed. Engl. 1995 (34), 1812.

[4] Vlatakis, G.; Anderson, L.I.; Muller, R.; Mosbach, K. Nature, 1993, 361, 645.

[5] Matsui, J.; Kato, T.; Takeuchi, T.; Suzuki, M.; Yokoyama, K. Anal. Chem. 1993 (65), 2223;

[6] Vlatakis, G.; Anderson, L.I.; Muller, R.; Mosbach, K. Nature, 1993 (361), 645;

[7] http://www.imprintedpolymer.com

[8] Kempe, M.; Mosbach, K. J. Chromatogr. A. 1994 (664), 276;

[9] Dickert, F. L.; Hayden, O. Trends in Anal. Chem. 1999 (18),3, 192.

[10] Sellergren, B.; Ekgerg, B.; Mosbach, K. J. Chromatogr. 1985 (347) 1-10.

[11] Anderson, L.I.; Mosbach, K, J. Chromatogr. 1990 (516), 313.

[12] Nicholls, I.A., Ramstrom, O.; Mosbach, K. J. Chromatogr. 1995 (691), 349-353.

[13] Cichna, M.; Knopp, D.; Niessner, R. Anal. Chim. Acta 1997 (339), 241.

[14] Sellergren, B.; Shea, k.J. J. Chromatogr. 1993 (635), 31.

[15] Kempe, M. Anal. Chem. 1996 (68) 1948.

[16] Spivak, D.A.; Gilmore, M.A.; Shea, K.J. J. Am. Chem. Soc. 1997 (119) 4388.

[17] Shea, K.J.; Spivak, D.A.; Sellergren, B. J. Am. Chem. Soc. 1993 (115) 3368.

[18] Yu, C.; Mosbach, K. J. Org. Chem. 1997 (62), 4025.

[19] Bruggemann, O.; Haupt, K.; Ye, L.; Mosbach, K. J. Chromatogr. A, 2000 (889) 15. 
[20] Kobayashi,T.; Wang, H.Y.; Fuji, N. Chem. Lett. 1995, 927.

[21] Wang, H.Y.; Kobayashi, T.; Fuji, N. Langmuir, 1996 (12), 4850-4856;

[22] Al-Kindy, S.; Badia, R.; Diaz-Garcia, M. Critical Reviews in Anal. Chem. 2000 (30) 4, 291-309;

[23] Wulff, G.; Haarer, J. Macromol. Chem. 1991 (192) 1329.

[24] Ye, L.; Weiss, R.; Mosbach, K. Macromolecules 2000 (33), 8239-8245.

[25] Ye, L.; Gormach, A.G.; Mosbach, K. Anal. Commun. 1999 (36), 35.

[26] Mosbach, K.; Ranstrom, O. Biotechnology, 1996 (140) 163-169.

[27] Vallano, P. T.; Remcho, V. T. J. Chromatogr. A, 2000 (888) 23-34.

[28] Kempe, M.; Mosbach, K. J. Chromatogr. A. 1994 (664) 276-279;

[29] Liang, C.; Peng, H.; Yao, S. Fresenius J. Anal. Chem. 2000 (367), 551-555.

[30] Lan Cao, Xi C. Zhou and Sam F. Li, Analyst, 2001(126), 184-188.

[31] Ji, H.; McNiven,S.; Ikebukuro, K.; Karubo, I. Anal. Chim. Acta. 1999 (390), 93100.

[32] Guilbault, G. G.; Jordan, J. M. Critical Reviews in Anal. Chem. 1988(19), 1-12.

[33] King, W. H. Anal. Chem. 1964 (36), 1735-39.

[34] Yan, Y.; Bein, T. Chem. Mater. 1992 (4) 975-977.

[35] Jarrett, M. R.; Finklea, H. O. Anal. Chem. 1999 (71), 353-357.

[36] Matsuura, K.; Ebara, Y.; Okahata, Y. Thin Solid Films, 1996 (273), 61-65.

[37] Yan, Y.; Bein, T. J. Phys. Chem. 1992 (96), 9387-93.

[38] Zimmermann, C.; Rebuere, D.; Dejous, C.; Planade, R. Sensors and Actuators B, 2001 (76), 86-94;

[39] Cai, Q.; Park, J.; . Zellers, E. T. Sensors and Actuators B, 2000 (62), 121-130. 
[40] Finklea, H.O.; Phillippi, M.A.; Lompert,E.; Grate, J.W. Anal. Chem. 1998, 70, 1268.

[41] Grate, J.W.; Patrash, S.J.; Abraham, M. H. Anal. Chem. 1995, 67, 2162.

[42] Harper, M. J. Chromatogr. A, 2000 (885), 129-151.

[43] Vahdat, N.; Swearengen, P.M.; Johnson, J. S.; Neidhardt,A. AM. IND. HYG. ASSOC. J. 1995 (56), 32-36;

[44] Wheeler, A.; Robell, A. J. Journal of Catalyst, 1969 (13), 299-305.

[45] Jonas, L. A.; Sevirbely, W. J. Journal of Catalyst, 1972 (24), 446-459.

[46] Dunkin, I.R.; Lenfeld, J.; Sherrington, D.C. Polymer, 1993, 34 (1), 77.

[48] Phillippi, Mark A Thesis, WVU.

[49] Luo, C.; Liu, M.; Mo, Y.; Qu, J.; Feng, Y. Ana. Chim. Acta. 2001 (428)1, 143148.

[50] Jarrett, Michael R. Thesis, WVU.

[51] Mayers, A. G.; Mosbach, K. Trends in Anal. Chem. 1997 (16)6, 321-331.

[52] Rosatzin, T.; Anderson, L.I.; Simon, W.; Mosbach, K. J. Chem. Soc. Perkin

Trans. 1991 (2), 1261.

[53] Shinar, R.; Liu, G.; Porter, M. D. Anal. Chem. 2000 (72), 5981-5987.

[54] McGill, R. A.; Abraham, M. H.; Gate, J.W. Chemtech, 1994 (9), 27-37.

[55] Grate, J.W.; Klusty, M.; McGill, R.A.; Abraham, M.H. Anal. Chem. 1992 (64), 610-624. 


\section{Chapter Two Design, Fabrication and Characterization of Preconcentrators (PCT) for Gas Phase Sensors}

\section{Introduction}

\subsection{Background}

Exposure to air contaminants in the atmosphere and in the workplace pose a threat to human health. Airborne contaminants originate from a variety of sources including: air pollution, food and beverage aromas, perfumes and cosmetics, solvent, chemical processes, gas leaks, cigarette smoke, off gases of processed materials, odorants, hidden explosives or drugs of abuse and chemical warfare agents [1]. Among the contaminants found in environments, volatile organic compounds (VOCs) have attracted considerable attention [2]. Measurement of the concentrations of VOCs in air is necessary in order to determine the sources and transport mechanisms of pollution, to study health effects and to determine compliance with regulated limits.

The concentration of VOCs in air can vary in both time and space, and measurement techniques must be designed to accommodate these fluctuations and to provide a result which can be used for the intended purpose. One method is to use near real-time instruments, such as infra-red spectrometer[3] or portable gas chromatographs or their detectors [4]. However, these instruments have limitations with respect to cost, stability, field calibration and power supplies which may detract from their use. Another method is to take a sample in the field and send it to a 
laboratory for analysis [5]. In this method, the integrity of the sample must be preserved through the trapping, transportation and analysis stages.

Many of the VOCs found in environment are present at very low parts per billion (ppb) and parts per trillion (ppt) levels (e.g., ethane, benzene, toluene) [6]. None of the routine analytical instruments available to date have adequate sensitivity to measure such concentrations directly. To identify and quantify these species, researchers must employ some sort of preconcentration methods prior to investigation in order to bring minimum detection levels within the range of regulatory concentration limits.

As the name suggests, the purpose of preconcentration is to enrich analyte molecules for improved efficiency in detection. The role of a preconcentrator is threefold; it focuses the sampled vapors by collecting them from a large volume of air and desorbing them into a smaller volume of air, it provides some resistance from baseline drift since the detector response from a desorbed pulse of vapor is generally rapid relative the time scale of drift, and it can reduce the amount of water vapor in desorbed samples by use of hydrophobic adsorbents.

In general, a preconcentrator adsorbs target analyte molecules from an inlet supply of air of relatively large volume flowing at a high rate, allowing those molecules of substances not of interest to pass through to an exhaust line. Then the adsorbing medium is heated quickly to desorb the analytes into a low airflow, which is directed into a detector for trace detection. (Figure 2.1). This process allows the target vapor analytes present in the large sample volume to be concentrated into a much smaller air volume before they are delivered to the detector, greatly increasing 
the probability of detection when using a concentration-sensitive detector such as an ion mobility spectrometer (IMS).
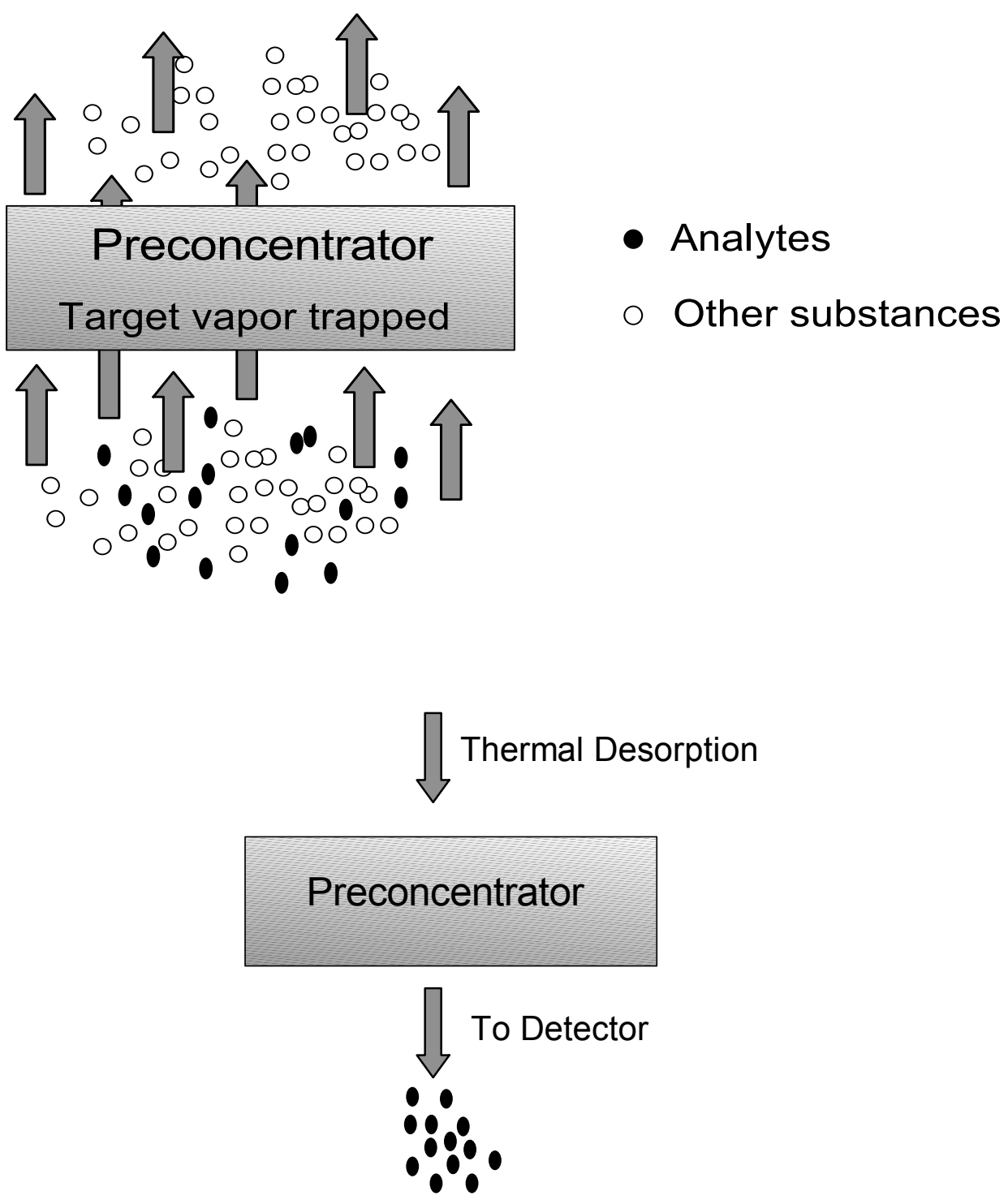

Figure 2.1 An operating scheme of a preconcentrator 


\subsection{Overview of Preconcentration Methods}

Preconcentration methods are common practice in analytical chemistry. Application examples can be found in trace analysis, water analysis, aromatic compounds analysis, in environmental analysis, air quality control, for soil samples, in odor analysis and in natural product investigations [7]. A summary of preconcentration methods of volatile organic compounds is given in Table 2.1. Advantages and disadvantages are listed according to operation in conjunction with a detection system. A distinction is made between the methods depending on the analyte phase. For the sampling from the gas phase, the cryogenic sampling and sorbent sampling methods are normally used. For the sampling from solid or liquid substances, preconcentration is mostly achieved by the purge and trap technique and the solid-phase extraction method.

\subsubsection{Purge and Trap Technique}

Purge and trap ( P\&T) has been used for decades to enrich VOCs from a solid or liquid matrix for introduction into a Gas Chromatograph (GC) for separation and identification. In this method, VOCs are extracted by purging an aqueous sample with either ultra-pure helium or nitrogen at a specified flow rate, temperature and time. This inert gas extracts the volatile analytes from the liquid matrix and transfers the volatile components to an ambient temperature trap containing different kinds of adsorbents (purge and trap). Subsequently, the VOCs are brought onto GC column by heating the sorbent trap. 
Table 2.1 Preconcentration methods

\begin{tabular}{|c|c|c|c|}
\hline \multicolumn{2}{|c|}{ Preconcentration method } & \multirow{2}{*}{$\begin{array}{l}\text { advantage } \\
\text { Simple setup }\end{array}$} & \multirow{2}{*}{$\begin{array}{l}\text { disadvantage } \\
\text { Water } \\
\text { condensation, } \\
\text { costly }\end{array}$} \\
\hline $\begin{array}{l}\text { gas } \\
\text { Phase }\end{array}$ & Cryogenic Trap & & \\
\hline & Thermal Desorption Tube & $\begin{array}{l}\text { High capacity, } \\
\text { mobile; preferential } \\
\text { sampling }\end{array}$ & $\begin{array}{l}\text { Only thermally } \\
\text { stable analytes }\end{array}$ \\
\hline $\begin{array}{l}\text { solid } \\
\text { or }\end{array}$ & Purge \& Trap (P\&T) & High sensitivity & $\begin{array}{l}\text { Long sampling } \\
\text { time }\end{array}$ \\
\hline \multirow[t]{2}{*}{$\begin{array}{l}\text { liquid } \\
\text { phase }\end{array}$} & Solid Phase Extraction (SPE) & $\begin{array}{l}\text { High } \\
\text { recovery/capacity }\end{array}$ & $\begin{array}{l}\text { Solvent removal } \\
\text { necessary prior } \\
\text { to analysis }\end{array}$ \\
\hline & $\begin{array}{l}\text { Solid Phase Micro-Extraction } \\
\text { (SPME) }\end{array}$ & Mobile sampling & $\begin{array}{l}\text { Low capacity, } \\
\text { low sample } \\
\text { volume }\end{array}$ \\
\hline
\end{tabular}


The major advantage of the P\&T technique is that all VOCs present in the sample can be transferred to the sorbent trap and consequently to the GC unit, resulting in low detection limits. The drawbacks of the P\&T method are twofold. First, it requires complex equipment, including a purge gas device, a sample vial and a sorbent trapping unit equipped with a heating device. When compared to normal liquid-liquid extraction techniques, the P\&T method results in longer analysis times [8]. The second disadvantage of this technique is related to the water vapor generated during the purging stage. Water vapor can interfere with the subsequent separation and detection. Normally, a water-removal trap is required for a P\&T system.

\subsubsection{Solid-Phase Extraction (SPE) and Solid-Phase Microextraction (SPME)}

Non- or semi-volatile components are usually isolated with solid phase extraction. Compared to liquid-liquid extraction, SPE is faster, uses less solvent, eliminates emulsions, and costs less. The simplest SPE method involves passing the sample through a packed cartridge that retains the analytes and possibly interfering sample components. Interfering components are washed off the packed bed and then the analytes are eluted. Elution can be done with a small volume of solvent to concentrate the sample, increase detection limits and simplify the analysis[9].

Solid-phase micro-extraction (SPME) is another approach adopted for field sampling [10], and has been increasingly used for the gas chromatographic determination of a wide variety of volatile and semivolatile organic compounds in water or aqueous samples. SPME fiber combines sampling and preconcentration in a 
single step. Basically, it involves extraction of specific organic analytes directly from aqueous samples, or from the headspace of these samples in closed vials, onto a fused-silica fiber coated with a polymeric liquid phase, poly(dimethylsiloxane) or polyacrylate (Figure 2.2). After equilibration, the fiber containing the absorbed or adsorbed analyte is removed and thermally desorbed in the hot injector of a gas chromatograph. The analytes are then analyzed by gas chromatography (GC) using an appropriate column and detector.

Both SPE and SPME techniques are very simple and fast, and do not employ any organic solvents either for sample preparation or cleanup. A further advantage of the headspace SPME approach is that samples from nearly any matrix can be analyzed since the fiber is not in direct contact with the sample. However, the SPME sample collection kit is not economical and the samples suffer losses during transportation if not cooled. In addition, SPME sampling can be time consuming and may suffer from limited sensitivity [9-11]. 


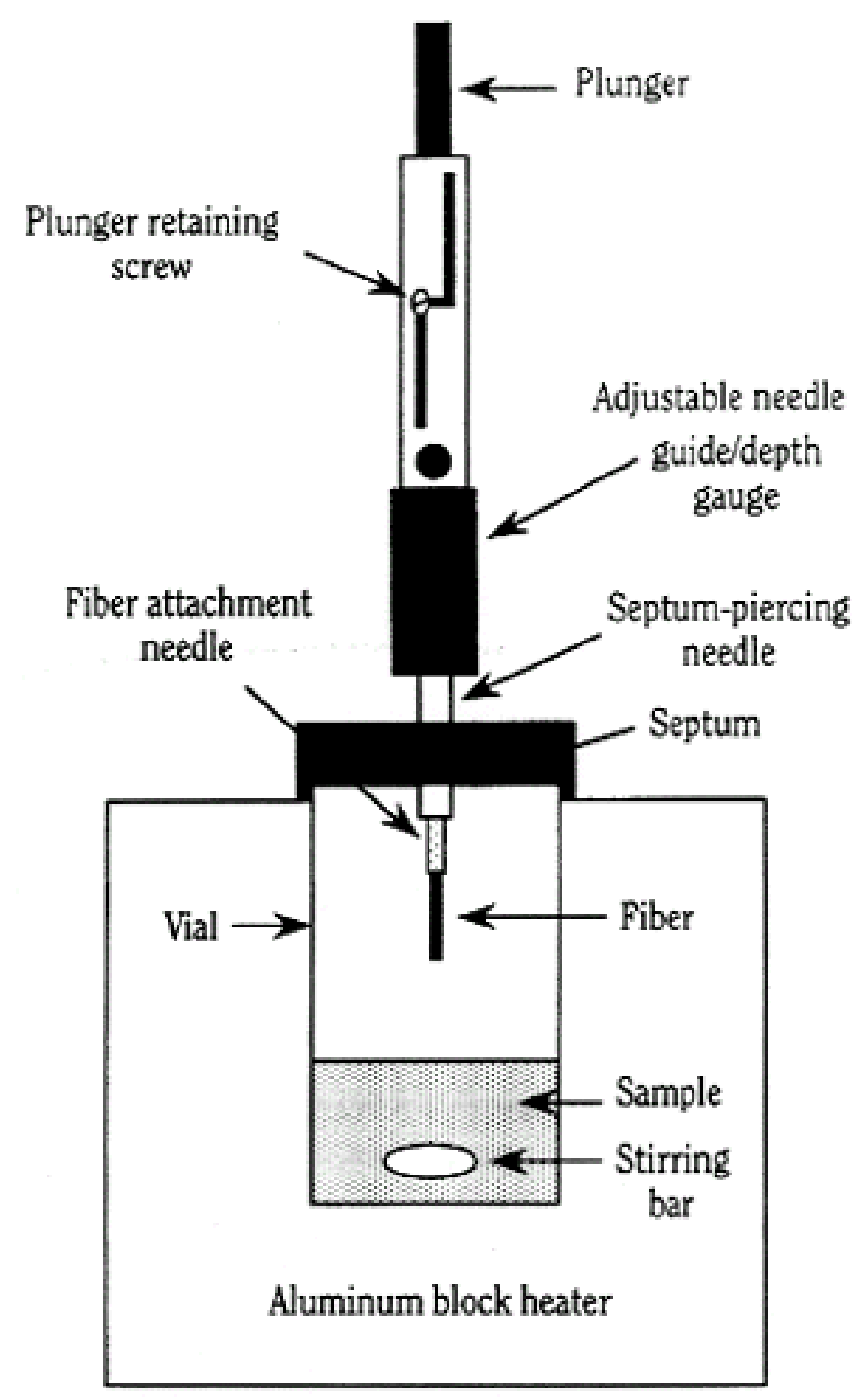

Figure 2.2 Schematic illustration of the headspace SPME method.

(Figure source from reference [11]) 


\subsection{3 "Cryo-focusing" Units}

The use of cryogenic trapping to concentrate VOCs prior to analysis has been established as a proven technique for VOC monitoring [12]. This method involves collecting the sample on an inert material (e.g., glass beads) at sub-ambient temperature. The temperature of the trap is below the condensation temperatures for trace VOCs but above the condensation temperature for major constituents of ambient air (e.g., nitrogen, oxygen). Conventional cryogens such as nitrogen or carbon dioxide are used to cool the sample collection trap. After collection, the trap that holds the solid particles undergoes rapid heating to have carrier gas flush the enriched VOCs onto a column for separation and detection. Typically the compounds are analyzed using a gas chromatography (GC) system (Figure 2.3)

This type of method is simple in principle, and is often preferred for its clean and complete thermal desorption. However, cryogenic preconcentration from humid air often leads to water condensation, which presents a major problem in the subsequent analysis. In addition, large amounts of cryogen are consumed during normal operations which results in added expense and the inconvenience of changing out cylinders. Safety is also a major concern when handling operations are carried out. 

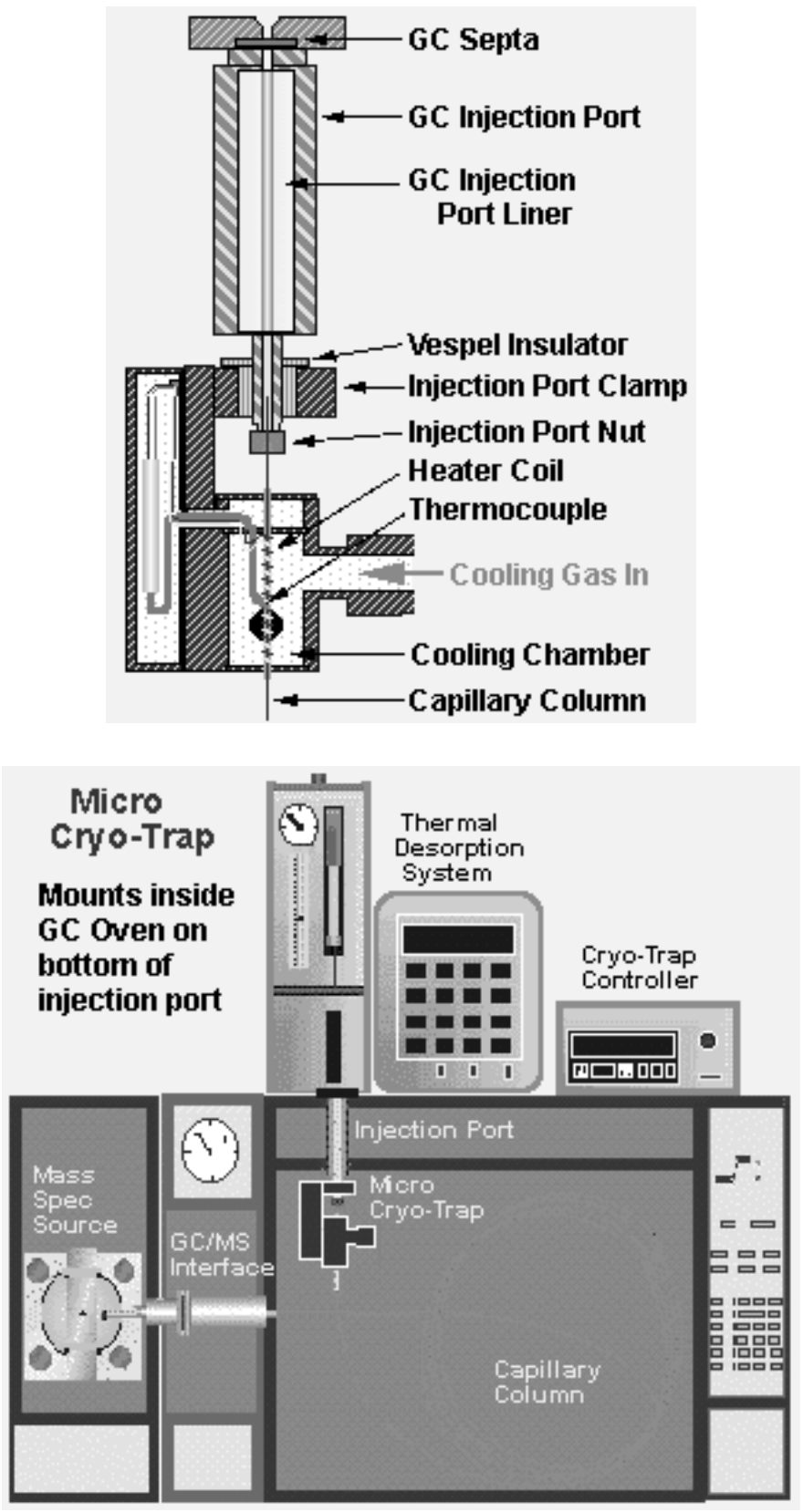

Figure 2.3 AutoDesorb $®$ Cryo-focusing system.

(Figure source from reference [13]) 


\subsubsection{Thermal Desorption Tube}

The use of an adsorbent bed to preconcentrate VOCs, followed by "flash" thermal desorption (heat rapidly to a temperature sufficiently high to desorb all the adsorbates) for injection into a gas chromatograph or a detector, is a standard technique for the analysis of trace gases in the atmosphere. In this method, sample uptake is achieved with a pump constantly drawing the sample through the packed tube. The trapping material is then heated and the analytes released at high temperatures (typically $200-300{ }^{\circ} \mathrm{C}$ ) are transported to the analytical column by the carrier gas. Thermal desorption allows the rapid and complete transfer of the entire sample to the GC column, resulting in maximum sensitivity. Therefore, the performance of the thermal desorption procedures is in many cases superior to liquid desorption. In addition, thermal desorption allows the preconcentration from large gas volume, which is important for trace analysis of air contaminants.

Thermal desorption tubes for vapor preconcentration have been reported recently. Short-path, multisorbent systems coupled to GC/MS, with detection limits on the scale of $0.05 \mathrm{ppb}$ by volume and with duplicates differing by less than $20 \%$ at this concentration levels have been described [14]. Mitra et al. have designed a microtrap-based injector system for continuous monitoring by gas chromatography of VOCs: a small adsorbent bed in series with the column is periodically rapidly heated to desorb the organics, then allowed to cool to concentrate once more the organics in the sample stream. The elution time for the organics from the column is less than the cooling time[15]. Lu and Zeller have described a miniature dual-adsorbent preconcentrator used in conjunction with a surface acoustic wave sensor to measure 
VOCs in the low ppb range [16]. For measurement at low levels, rejection of interference by water vapor is an important consideration. The problem has been overcome by the use of an adsorbent such as Tenax which has low water retention, by use of a dry air purge before desorption of the VOCs and by control of the adsorption temperature. Preconcentrator tubes containing Tenax operated with periodic temperature pulses for desorption have been used in conjunction with a sensor array of quartz crystal microbalances (QCMs)and a pattern recognition system to identify toxic compounds present at low levels in air [17]. A similar system, which also employed an intermediate dry air purge step prior to thermal desorption to decrease significantly the limits of detection, has been described for analysis of organic vapors in exhaled breath [18]. Nakamoto and coworkers described an odor-sensing system using a QCM array and a Tenax-based preconcentrator with variable-temperature desorption [19]. In the desorption cycle, the preconcentrator was stepped to two different temperatures, therefore achieving a further element of separation of the analytes additional to that given by the QCM array, based on the range of desorption temperature of different types of analytes. For ketones and esters adsorbed on Tenax, the desorption temperature was strongly influenced by the boiling temperature of the adsorbent. Nakamoto and coworkers described a method of automatic adjustment of the desorption temperatures so as to maximize discrimination between different components of a test sample. The stability of Tenax at temperature up to $260{ }^{\circ} \mathrm{C}$ in air was also noted from this work. 


\subsection{Adsorbent Material}

Most of the techniques presently used for the preconcentration of VOCs in gaseous samples are based on adsorption of the analytes of interest on a suitable preconcentration material followed by thermal desorption. Adsorbents used for preconcentration are summarized in Table 2.2. Commonly used materials include carbon-based materials such as activated carbon [20] and molecular sieves[21]. Some of the sorption materials listed are also used in chromatography as stationary phases, e.g. polydimethylsiloxane (PDMS) and its derivatives. Sampling tubes packed with organic polymers such as the Tenax adsorbents (Tenax GR, Tenax TA) have been widely used to collect hazardous compounds present at very low concentration in air[22]. The standard methods use Tenax as sorbent material, but this is not suitable for very volatile or very polar compounds because of its low capacity for these compounds. In addition, Tenax sorbents do not show specific selectivity.

In thermal desorption, blanks are caused by the adsorbent itself. At the high temperature used, adsorbents tend to break down (especially polymeric ones), resulting in characteristic degradation peaks [23]. New adsorbents that have a higher adsorption capacity for organic compounds may allow for better adsorption and desorption of pollutants in air. 
Table 2.2 Common adsorbent materials

\begin{tabular}{|c|c|c|c|}
\hline Sorbent & Brand Name & Properties & Application \\
\hline \multirow[t]{3}{*}{ Porous polymer } & Tenax TA & $\begin{array}{l}\text { 2,6-diphenyl-p- } \\
\text { phenyleneoxide, stable } \\
\text { up to } 300^{\circ} \mathrm{C} \text {, low affinity } \\
\text { to polar substances }\end{array}$ & $\begin{array}{l}\text { Volatile and semi- } \\
\text { volatile substances }\end{array}$ \\
\hline & Tenax GR & $\begin{array}{l}\text { Lower surface area than } \\
\text { Tenax TA }\end{array}$ & $\begin{array}{l}\text { Volatile and semi- } \\
\text { volatile substances }\end{array}$ \\
\hline & chromosorb & $\begin{array}{l}\text { Styrene-divinylbenzen } \\
\text { polymer, stable up to } \\
250^{\circ} \mathrm{C}\end{array}$ & $\begin{array}{l}\text { Small molecules in } \\
\text { air }\end{array}$ \\
\hline Active Charcoal & & $\begin{array}{l}\text { High surface area, less } \\
\text { hydrophobic, thermally } \\
\text { stable }\end{array}$ & $\begin{array}{l}\text { Small molecules in } \\
\text { air, high boiling point }\end{array}$ \\
\hline Graphite Carbon & Carbotrap & $\begin{array}{l}\text { Non-porous, } \\
\text { hydrophobic, variable } \\
\text { particle size }\end{array}$ & $\begin{array}{l}\text { Air contaminants } \\
\text { C4,5-C9-30 }\end{array}$ \\
\hline $\begin{array}{l}\text { Rubbery } \\
\text { polymer, } \\
\text { GC stationary } \\
\text { phases }\end{array}$ & $\begin{array}{l}\text { PDMS and its } \\
\text { functional } \\
\text { derivatives }\end{array}$ & Variable polarity & $\begin{array}{l}\text { Polar and semi- } \\
\text { volatile substances, } \\
\text { high molecular } \\
\text { weight }\end{array}$ \\
\hline Silica gel & Porasil & & Polar analytes \\
\hline Molecular Sieve & & $\begin{array}{l}\text { Surface area : } 5-100 \\
\mathrm{~m}^{2} / \mathrm{g}\end{array}$ & $\begin{array}{l}\text { Specific adsorption } \\
\text { depend on pore } \\
\text { shape and size }\end{array}$ \\
\hline
\end{tabular}




\subsection{Preconcentrator Project Description}

Prototype instruments equipped with small thermally desorbed preconcentrators coupled to microsensors or microsensor arrays have been reported [24]. Typical designs comprise a thin-walled glass or metal tube, 1-5 $\mathrm{mm}$ in diameter, packed with a granular porous adsorbent material and wrapped with a resistive heater coil. The preconcentrator design is a small sorbent trap. The sample passes continuously through it, and periodic electrical heating releases the adsorbed analytes as a "enriched pulse", which serves as an injection for the detection system. It is obvious that the sensitivity of a detector (sensor) can be enhanced by providing such on-line preconcentration.

However, one issue of concern is non-selective adsorption of many substances from the gas phase by the preconcentration unit. Selective adsorption can be regulated by choosing the specific adsorbents with the suitable pore size, sorption capacity and particle size. As with our previous work in Chapter I, the imprinted polymers exhibited higher sensitivity and selectivity toward small aromatic molecules than the traditional adsorbents such as Tenax TA and GR. In this project, we intend to incorporates such selective adsorbents into the preconcentrator to enhance the selectivity.

The key to success is careful construction of the preconcentrator unit, the adsorption/desorption protocol and choice of adsorbents. On the practical side, the preconcentrator design must possess short diffusion paths, low thermal mass and good thermal conductivity for rapid heating/cooling and low power consumption. Important design criteria for the preconcentrator also includes low dead-volume 
geometry, which requires minimized unit components. In order to achieve detection limits in the ppb range while minimizing desorption bandwidth, the size of the preconcentration tube must be limited.

The analyte vapor is monitored using a FID. The enrichment factor (preconcentration factor) is used to evaluate the preconcentrator performance.

The present study is undertaken to examine the preconcentrator design and operation in more detail, to determine if the detection limits and linear dynamic ranges could be improved, and to expand the set of adsorbents used in preconcentration. 


\section{Experimental Section}

\subsection{Synthesis of Polypyrrole}

Preconcentrators based on polypyrrole powders and polypyrrole films were investigated for the enrichment of aromatic compounds. Among the various conducting polymers studied, polypyrrole (PPY) and its derivatives have been one of the most widely used classes of conducting polymers of the past decades. Preparation methods for polypyrrole have been intensely researched and the stable polymer can be conveniently prepared on various substrate materials from organic or aqueous media by electrochemical or chemical methods. Our interest is to use PPY as a sorbent for vapor preconcentration. For example, it should extract aromatic compounds through the $\pi-\pi$ interactions [24]. Its conductivity may also be useful for resistance heating of a preconcentration stationary phase.

\subsubsection{Polypyrrole Powder Synthesis}

\section{a. Choride-doped Polypyrrole- PPY (I)}

Pyrrole was added to the aqueous iron(III) -chloride solution (the molar ratio of oxidant to pyrrole was 2.25 ). The mixture was well stirred, and the polymerization was allowed to proceed at room temperature overnight. The resulting powder were filtered, and washed abundantly with water until the filtrate was clear, and then dried in a oven at $110^{\circ} \mathrm{C}[25]$. 


\section{b. p-Toluene Sulfonate-doped Polypyrrole- PPY (II) [26]}

Ferric Ethylbenzenesulfonate: To an aqueous solution of $10 \mathrm{~g}(0.037 \mathrm{~mol})$ of $\mathrm{FeCl}_{3}$ in $140 \mathrm{~mL}$ water, a solution of $\mathrm{NaOH}(0.11 \mathrm{~mol})$ was added with vigorous stirring. The precipitate was washed twice by adding water, stirring, allowing the solids to settle, and siphoning off the supernatant. The brown ferric hydroxide was collected on a glass filter and washed with water. All the precipitate was transferred to $250 \mathrm{~mL}$ methanol. The suspension was added $0.1 \mathrm{~mole}(19.2 \mathrm{~g}) \mathrm{p}$-Toluene-sulfonic acid and the mixture was stirred for $5 \mathrm{hrs}$ at $45{ }^{\circ} \mathrm{C}$. The solution was filtered, and the solvent was moved on a rotary evaporator. The resulting oil was dried in a oven at $110^{\circ} \mathrm{C}$. The solid residue was then ground into a powder.

Polyrrole p-Toluene Sulfonate: Polypyrrole was prepared by the reaction of ca. 2.25 equivalents of the ferric salt with 1 equivalent of pyrrole monomer in methanol solution. The reaction mixture rapidly darkened and produced a thick black suspension. The reaction was continued for $3 \mathrm{hrs}$ and the polypyrrole precipitate was isolated by filtration and rinsed thoroughly with methanol followed by acetone. The powder was dried at room temperature.

\subsubsection{Polypyrrole layer onto Nichrome Ribbon}

Polypyrrole film was coated directly onto a Nichrome ribbon $(7 \times 0.2 \mathrm{~cm})$ by a chemical polymerization method [27]. The Nichrome ribbon was pretreated by

dipping in dilute $\mathrm{KMnO}_{4}$ solution to remove any oil. Pyrrole $\left(65 \mu \mathrm{L}, 9.24 \times 10^{-4} \mathrm{~mol}\right)$ was injected into a sample bottle containing a stirred solution of $1.17 \mathrm{~g}$ $\mathrm{Fe}\left(\mathrm{NO}_{3}\right)_{3}\left(2.91 \times 10^{-3} \mathrm{~mol}\right)$ (molar ratio: $\left.1: 3.2\right)$ in $50 \mathrm{ml}$ deionized water. The pre- 
treated Nichrome ribbon was immersed in the solution during the polymerization. Polymerization was allowed to proceed at room temperature overnight. The coated ribbon was washed with water and methanol and dried at $65^{\circ} \mathrm{C}$ in air. A fine dark gray coating with mass in the range of $20-30 \mathrm{mg}$ was obtained on Nichrome ribbon. 


\subsection{Vapor Atmosphere}

Test atmospheres were generated by the vapor generator described in Chapter I. Tedlar bags (SKC) were used to collect and contain vapors with low concentrations. The Tedlar bag is manufactured from polyvinyl fluoride (PVF) film and furnished with a polypropylene valve and a septum holder. The bags are generally considered to be inert and can be used to collect samples containing vapor of common solvents, hydrocarbons, chlorinated solvents, and many other classes of compounds.

Test atmospheres of the low concentration (low ppm level to ppb level) vapors were prepared by introducing a known volume of vapor with a defined concentration into a seasoned (i.e. pre-exposed and purged) 5-liter Tedlar bag and diluting with a known volume of clean, dry air. After mixing by physical agitation, the bag was attached to the inlet value of the instrument using a short section of narrow-bore Teflon tubing. All bags were seasoned by pre-exposure to low concentration of the test compounds followed by repeated flushing with clean air. Aliquots were withdrawn with a gas-tight syringe, injected into each of several additional bags, and diluted as necessary to obtain atmospheres spanning a range of lower concentrations.

\subsection{Preconcentrator (PCT) Construction}

A schematic diagram of the preconcentration assembly used in this study is presented in Figure 2.4. The goal is to construct a compact, low power thermodesorption system for possible integration into a sensor system as an additional module. The principle of a preconcentrator unit is a stage that samples and collects analytes from an inlet gas sample stream and ejects them on command into the 
separation stage. The preconcentrators are put on-line with the sample stream. The vapor analyte is introduced into the FID detector through the preconcentrator. The analyte is trapped in the polymer film/powder and thermally desorbed by electrical heating. The desorption is achieved by resistive heating by a current pulse in a few seconds. Rapid desorption is essential for producing a sharp concentration pulse.

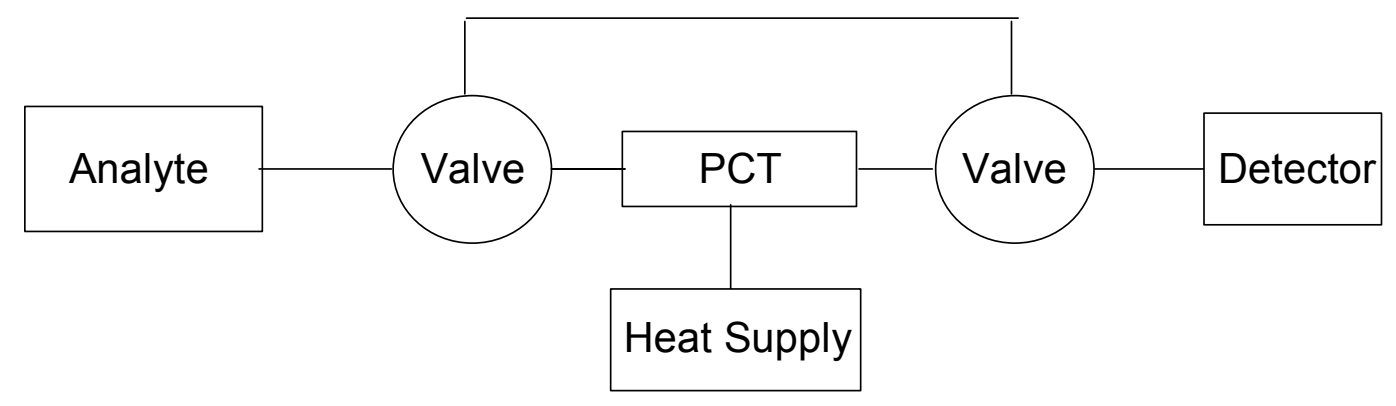

Figure 2.4 A schematic diagram of a PCT assembly

\subsubsection{Preconcentrator Design I}

This design was used to investigate performance of a preconcentrator based on PPY films. Figure 2.5 shows the key components of this unit. The heating element in this unit was a thin Nichrome ribbon. The preconcentration was done on a thinlayer PPY coating deposited on the ribbon. PPY film was coated onto the ribbon by a chemical polymerization method as described previously. The PCT unit was fabricated by a piece of ceramic block $(8 \times 1 \times 0.5 \mathrm{~cm})$. The main body of the block was machined to generate a channel in the center, which is $6 \mathrm{~cm}$ long and $3 \mathrm{~mm}$ wide. On each end of the channel, a small hole was drilled through the bottom of the block to 
allow for electrical connection. The left and right sides of the block were drilled to form connection to the channel; the drilled holes supported stainless steel screw fittings to attach Teflon tubes. During assembly, each end of the coated Nichrome ribbon was press-fitted into a slit located on the top of a copper wire electrode. The suspended ribbon was placed into the flow channel with two electrodes sitting through the holes on the bottom.

A glass cover was compressed against the surface of ceramic block to seal the flow channel by using clamps on opposite sides. The rubber O-ring made a pressure seal between the copper electrode and the bottom base of the silicon block. To avoid leaking, the holes in the bottom was also sealed careful with an adhesive which can stand high temperatures. The assembled unit was tested for gas leaks by soap solution before using. 

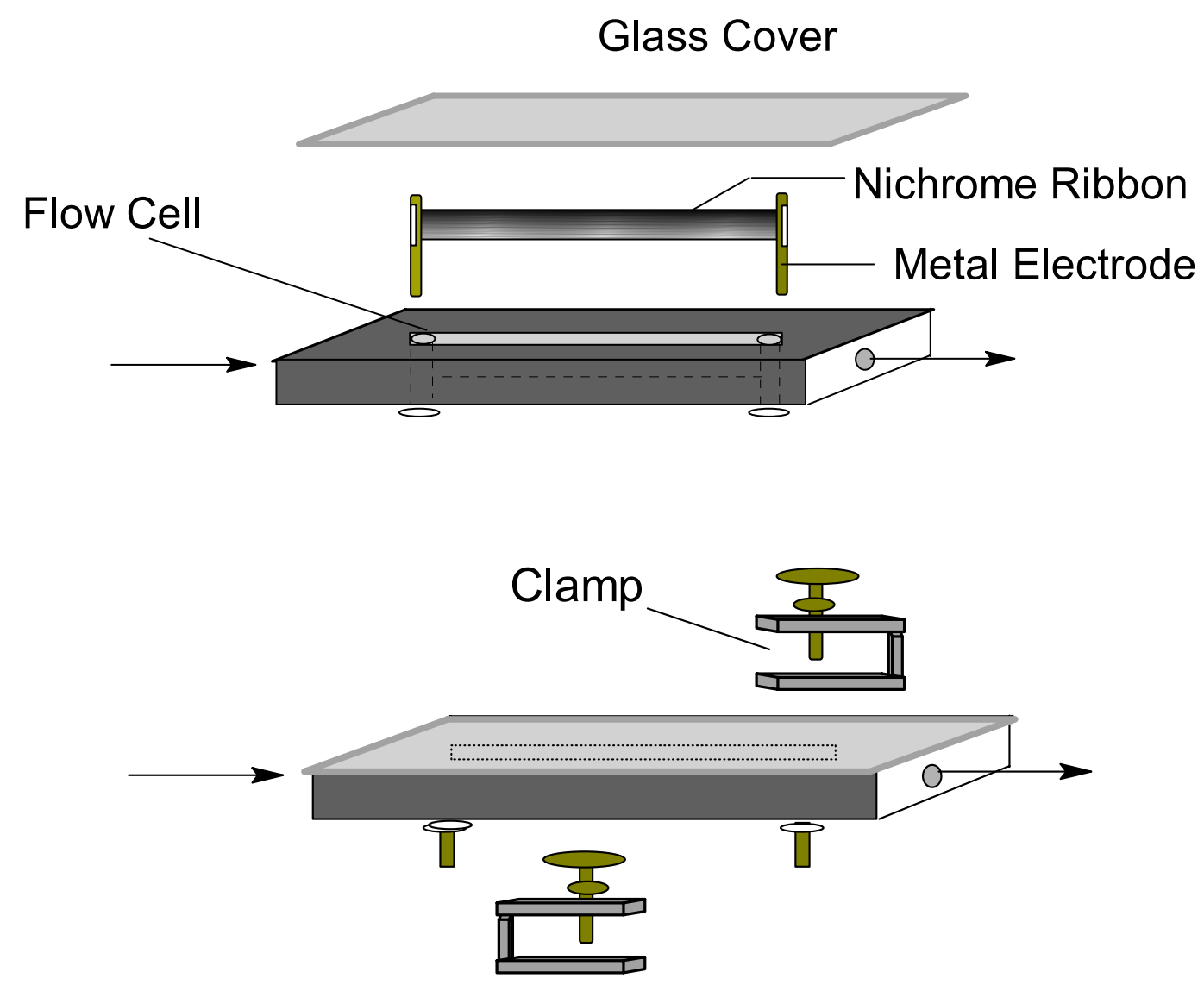

Figure 2.5 Preconcentrator Type I 


\subsubsection{Preconcentrator II}

The preconcentrator system layout is shown in Figure 2.6 (a) This preconcentration unit was built on a Teflon block $(14 \mathrm{~cm} \times 2.5 \mathrm{~cm} \times 1 \mathrm{~cm})$. The adsorbent tubes were custom-made of glass, $7 \mathrm{~cm}$ in length, $4 \mathrm{~mm}$ o.d. and a wall thickness of $\sim 1 \mathrm{~mm}$. The Teflon block was machined to create a groove ( $1 \mathrm{~cm}$ wide) in the center. A pair of copper cubes were mounted on the opposite ends of the groove. The glass tube was pressed tightly into the holes on the sides of copper cubes. The glass tube was connected to the metal tubing (1/8") attaching to the front sides of the cubes.

This system enabled the use of two types of heating elements, the Nichrome ribbon and the fine Nichrome wire $(1 \Omega / \mathrm{cm})$. A Nichrome ribbon coated with PPY layer was inserted directly into the adsorption tube. In order to make a good electrical contact, each end of Nichrome material ribbon was extended through an opposite opening of the copper cube and was fastened to the metal connector next to the cube. In another design, when the tube was packed with adsorbents, a Nichrome wire coil (40-cm total length) was placed through the core of the adsorbent bed (Figure 2. $6 \mathrm{~b}$ ) .

Except for the PPY film, the adsorbent materials used in this investigation were synthesized PPY powders, synthesized MIP particles and commercial adsorbents such as Tenax TA and Tenax GR. The PCT tubes were filled with solid adsorbents up to a bed length of $7 \mathrm{~cm}(10 \sim 100 \mathrm{mg})$ for sampling. Glass fiber was used to fit both ends of the tube and to hold the adsorbents in place. 
The system was attached to the vapor generation system and gas pressure was used to drive the analyte through the trap. A glass Y-connector at the system inlet was used to switch between the vapor atmosphere and the clean dry air source without opening the system. 


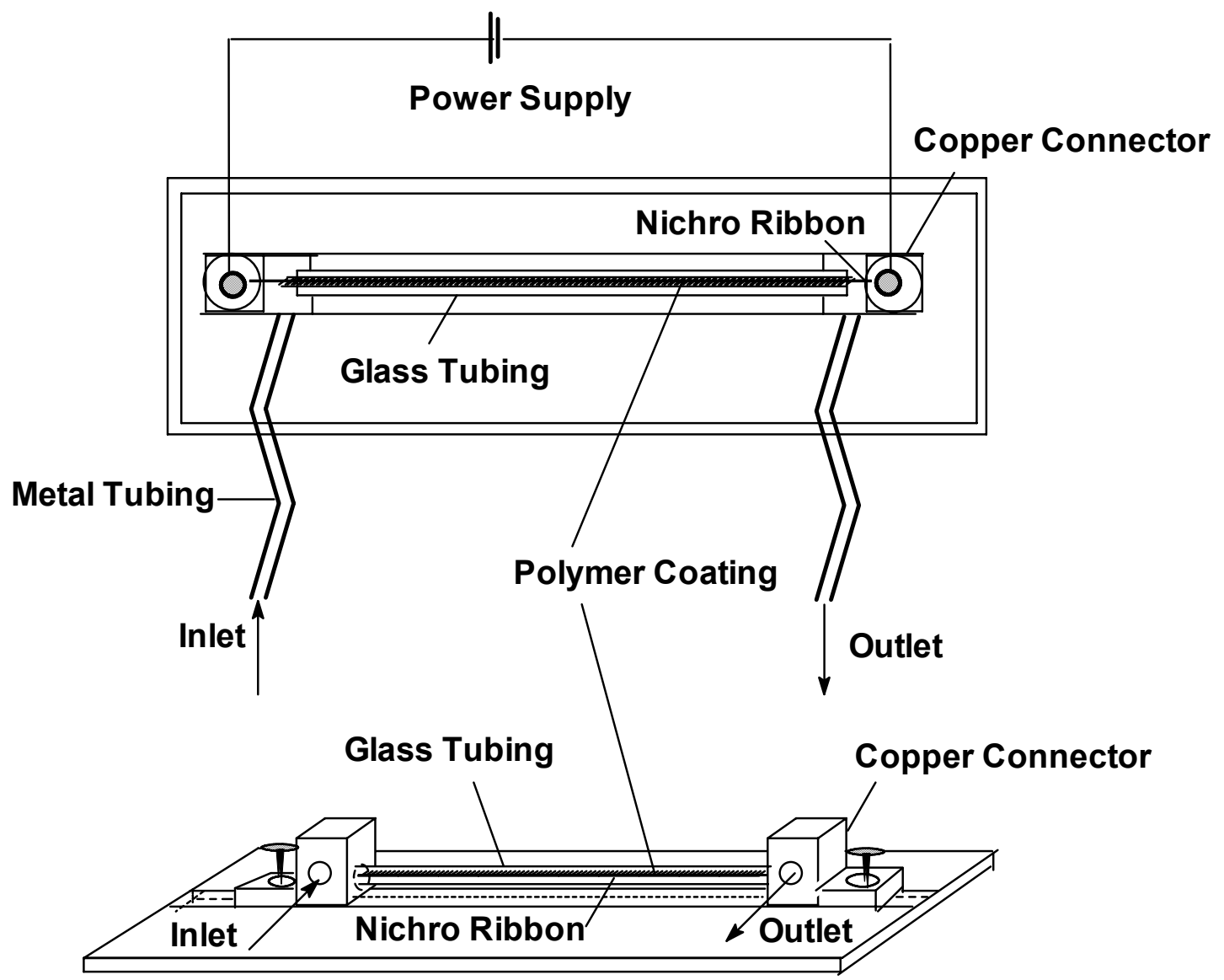

(a)

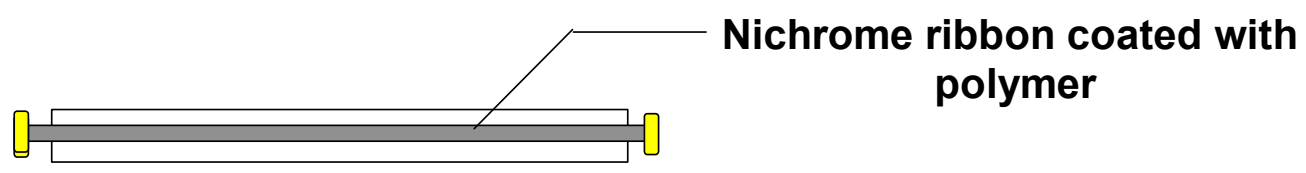

Nichrome wire

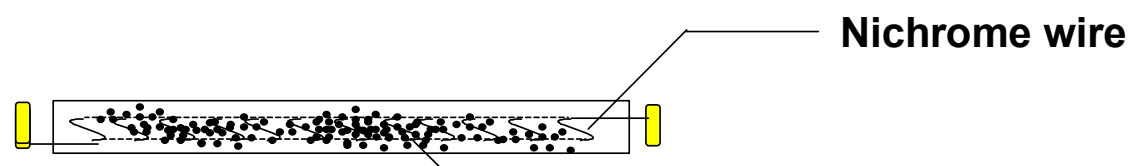

Packed adsorbent

(b)

Figure 2.6 Preconcentrator type II 


\subsubsection{Preconcentrator Design III}

This type of preconcentrator unit is shown in Figure 2.7 together with enlarged sections of its major components. The system is similar to the design reported by Zellers [16]. The preconcentrator unit was constructed from thin-walled glass capillaries (Fisher, o.d.: $1.7 \mathrm{~mm}$, i.d.: $1.2 \mathrm{~mm}$ ) with a length of $7.5 \mathrm{~cm}$. The mass of adsorbent packed into the tube ranged from 5- $20 \mathrm{mg}$. The adsorbents was held in place with glass fiber filter (Fisher). A type K thermocouple (Omega Engineering) was placed against the outer wall of the glass capillary and held snugly in place under a sleeve of polyimide (i.d.: $1.8 \mathrm{~mm} .0 .05 \mathrm{~mm}$ wall thickness, Cole Parmer). A $35-\mathrm{cm}$ section of Nichrome wire with resistance of $40 \Omega$ was coiled around and attached to the polyimide tube with insulation adhesive (Minnco) to create a heated region that extended beyond the length of the adsorbent bed. The same Nichrome-wire-wrapped polyimide tube could be used repeatedly. A PID (proportional integral derivative) controller (Minco) was assembled to control the heating profile for thermal desorption. The PID controller can regulate the power supply so that the actual temperature on the trap sensed by the thermocouple closely matched the prescribed temperature ramp. In the experiment, the controller was adjusted to ramp from the room temperature to the desorption set-point temperature within 8 seconds with less than a $15{ }^{\circ} \mathrm{C}$ overshoot in all cases. A series of the thermocouple tests indicated that equivalent readings were obtained from placement outside or inside the adsorbent tube.

Teflon tubings (o.d.:0.3 cm) were pressed fit into the ends of the PCT tube. A sleeve of silicon-rubber tubing was clamped around the joint of the Teflon and PCT 
to prevent leaks. The PCT assembly was connected to the system sampling trains with SwageLock fittings placed on the end of the Teflon tubing opposite from the glass/Teflon tube interface. The transfer lines in the unit were $1 / 16$ " od. Teflon tubings in order to reduce dead volume.

The diaphragm pump was used for the preconcentrator sample collection. The diaphragm pump drew a sample through the preconcentrator at an adjustable flow rate. The low-dead-volume six-port valve switched the preconcentrator between the sampling and desorption positions. The preconcentrator was connected to the six-port valve via $1 / 16$ " id Teflon tubing, which allowed thermal desorption flow in the reverse direction of sampling flow (i.e. back flushing) to avoid band broadening or irreversible trapping of the less volatile compounds on the higher-surface area downstream adsorbents. (Figure 2.8)

In this setup, the glass capillaries with small i.d. were used. The sorption material required careful packing. Otherwise, a pressure loss over the trap resulted from clogged sorption material when heated. This then altered the signals of the tested gases and desorption kinetics were altered unfavorably. 

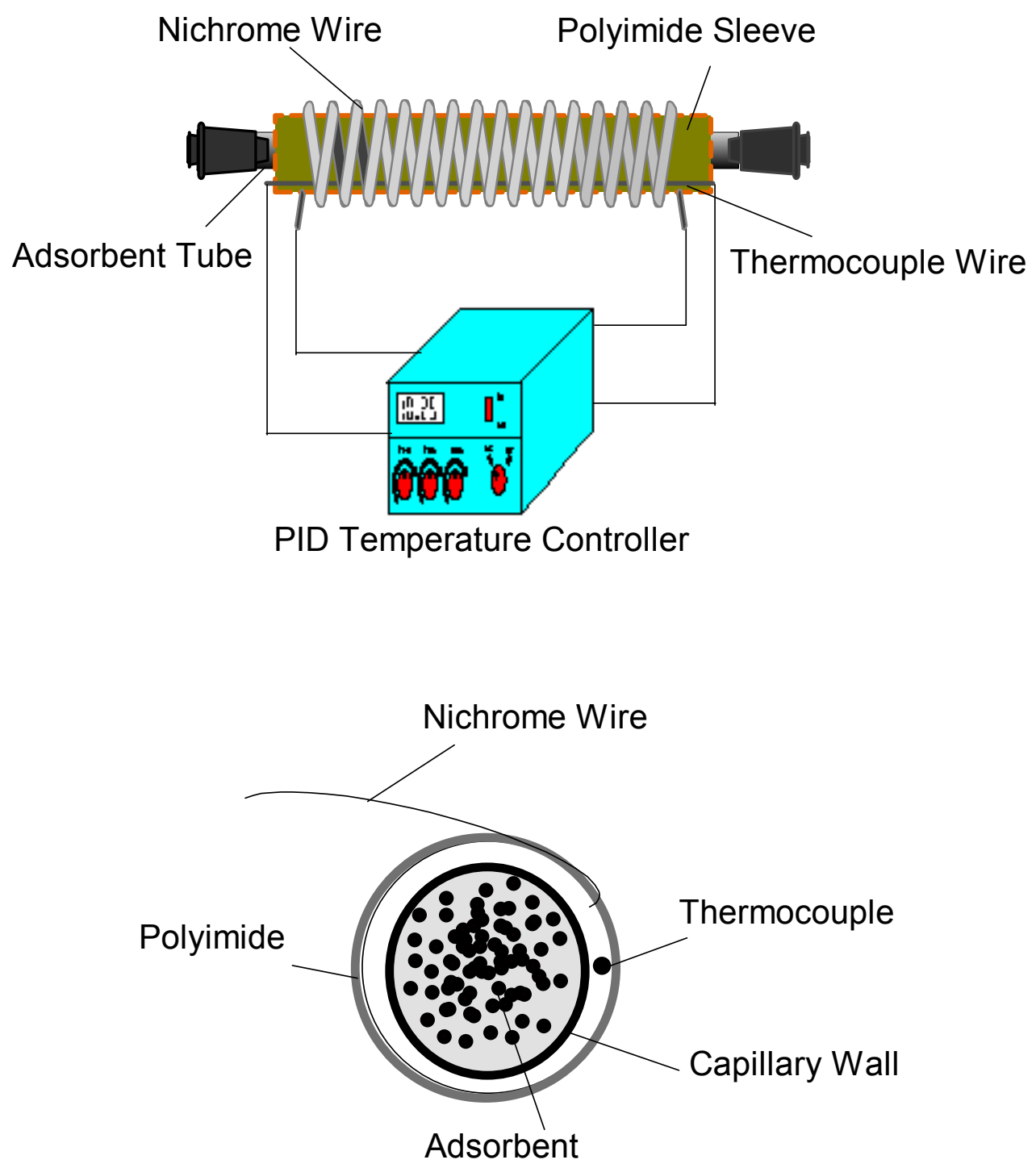

Figure 2.7 Preconcentrator Type III 


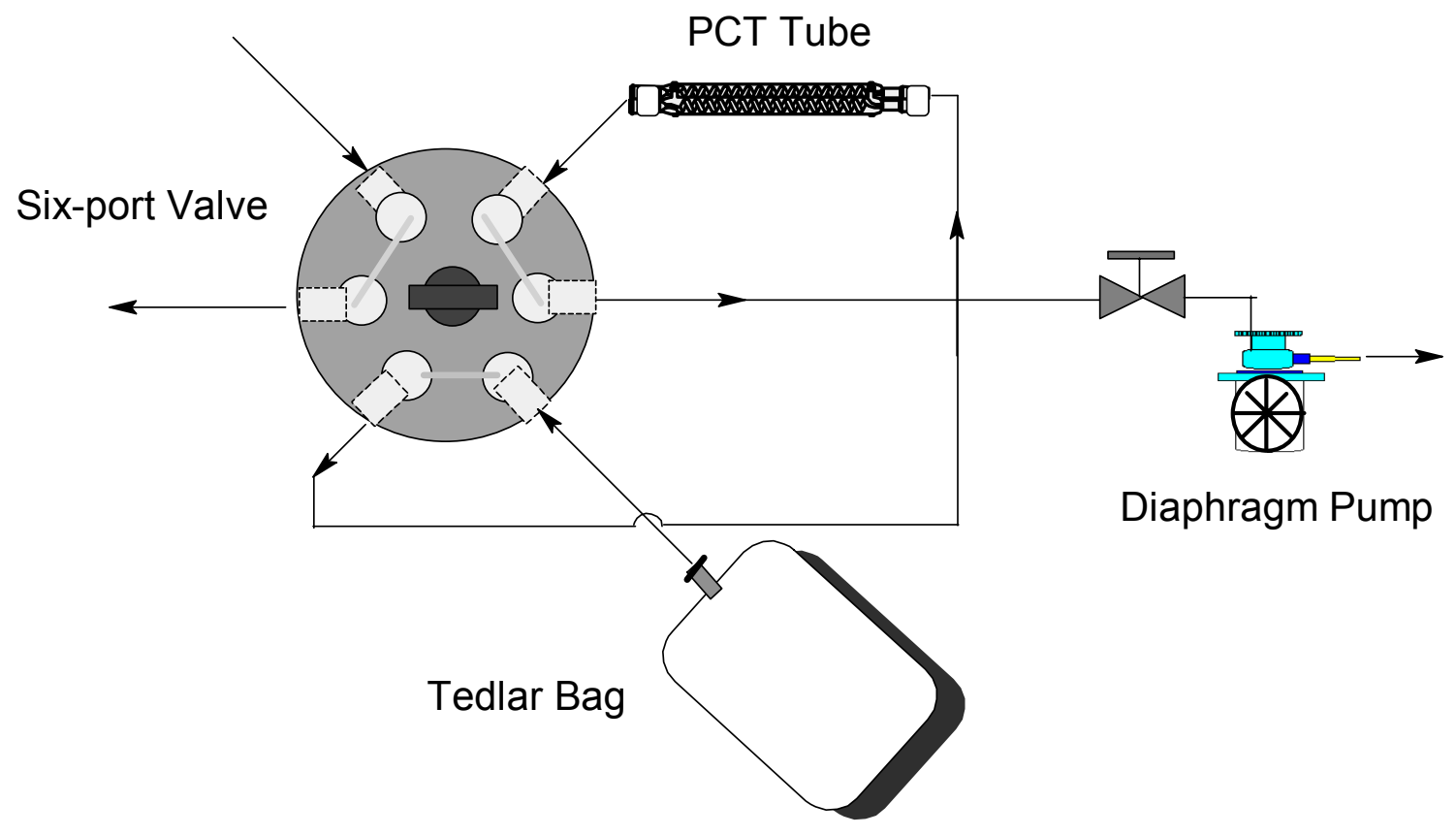

a. Sampling

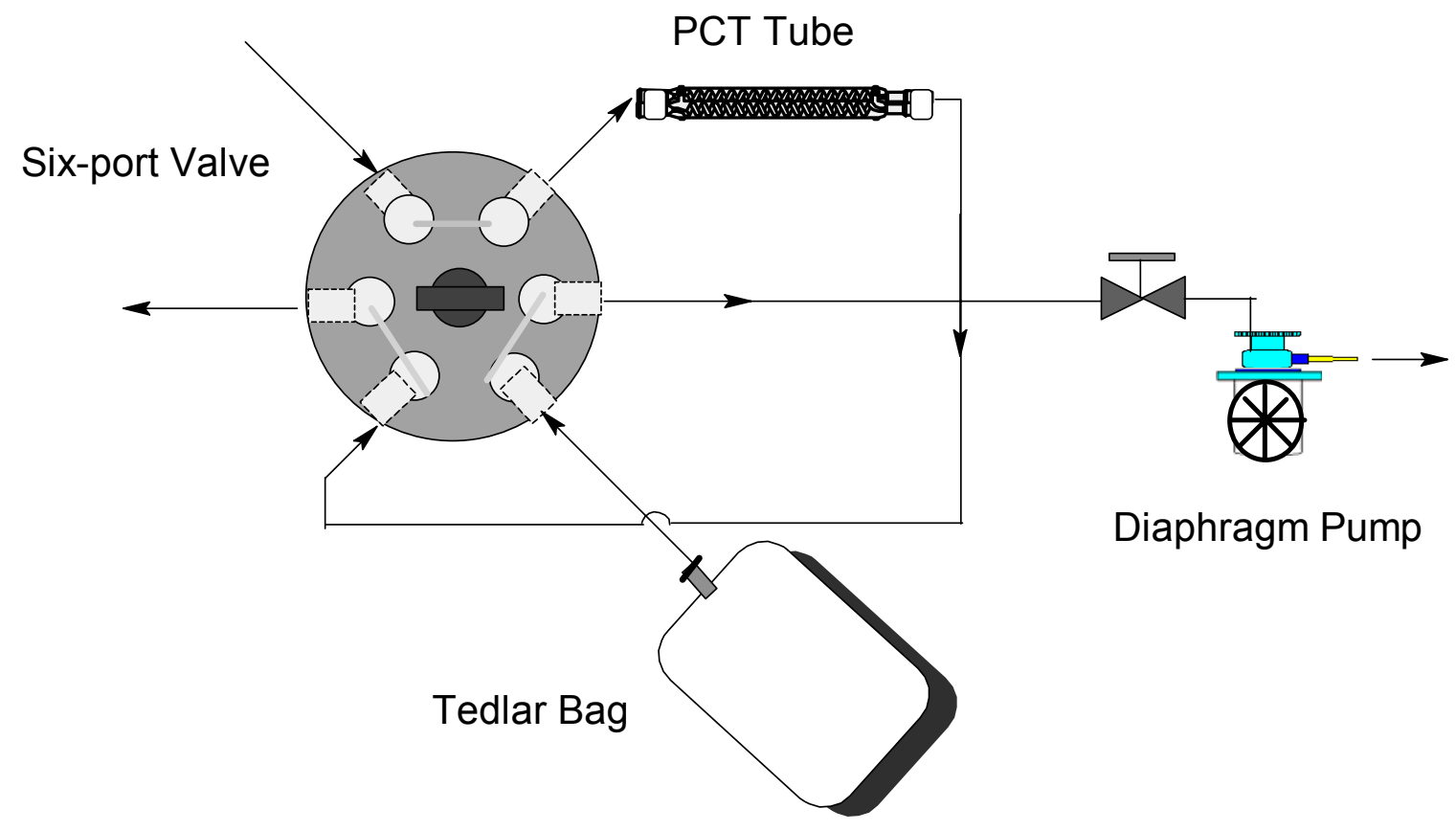

b. Desorption

Figure 2.8 A schematic assembly of Preconcentrator Type III 


\subsection{Operation Mode}

A complete analytical cycle consisted of a sequence of three operating modes.

Sampling mode. The analyte stream was introduced into the adsorbent unit at a constant rate for a predefined sampling period. The preconcentrator retained the incoming vapors.

Desorption mode. Application of a pulse of electrical current across the $\mathrm{NiCr}$ wire heated the preconcentrator to a set temperature. The preconcentrator was flash heated and the plug of desorbed vapor passed to the FID detector. The heater in the tested setups required a power supply of up to $20 \mathrm{~V}$ to heat the trap rapidly. For this study, the maxima of the detected peaks were used for quantification.

Recovery mode. After the FID signal was recorded, the instrument was switched to recovery mode where clean, dry air was again directed over the preconcentrator tube to re-established the baseline. In some cases, switching from regeneration mode to baseline mode gave a large spike in the output signal owing apparently to a pressure surge associated with actuating the valve.

Two types of protocols were used in the experiment.

1) Continuous sampling. The sample stream was connecting to the FID through the PCT. Heating the PCT tune released the sorbed vapor, which was then carried by the sample air to the FID for detection. 
2) Indirect sampling. The PCT collected the samples for a fixed interval.

During this time, the FID was in direct sampling mode (i.e. exposed to sample air that is delivered to the FID without passing though a PCT). Then the valve was switched so that the FID was exposed to air that was routed through the PCT. A slight perturbation in FID baseline occasionally occured during valve switching. After 10 seconds for baseline stabilization, thermal desorption was begun and continued for 30 seconds. A sharp desorption peak appeared. Then the valve was switched back to collection mode. 


\section{Results and Discussion}

Several manually operated preconcentration designs with different configurations were built in order to optimize the dimensions and operation parameters. The Type I prototype was built at the very beginning of the project and was used to examine the preconcentration performance for Polypyrrole (PPY)film. However, backpressure is a big issue in this design since the dimension of the flow channel in this unit is much smaller than that of the transportation train. The Type I prototype was modified into Type II and III units. The type III unit was designed to provide better temperature control during the heating phase and to reduce to dead volume. The latter two units are mostly discussed in this section. Detailed results from tests with PPY and Tenax polymers are presented first to illustrate several points about the performance of the preconcentrator units. Molecularly imprinted polymers are also tested as the adsorbents. The FID has a high sensitivity toward toluene vapor, one of the targeted small aromatic analytes discussed in previous section, so this section mainly focuses on the response of toluene vapor. Steady streams of vapors are generated by the modified Kintek permeation system.

\subsection{Heating Characteristics}

The key component in the preconcentrator device is the heating element embedded in the unit. The characteristics of the different heating elements in PCT designs were studied. The thermal desorption temperature depends upon several factors such as heater design, the applied power and heat dissipation, etc. 
The different desorption temperatures in various PCT designs are achieved by increasing either the voltage across the Nichrome material or the heating duration. Typical time of the resistant heating is in the range of 10-30 seconds. In the initial designs of preconcentrators ( type I and II ), no thermocouple is attached to the PCT unit and a series of experiments are carried out to pre-determine the approximate temperatures reached at different power levels. The temperature curves obtained under a fixed heating interval are plotted in Figure 2.9 and Figure 2.10. For a given power setting, the final temperature reached is highly reproducible. The temperature reported here is the maximum temperature measured on the PCT wall surface, and not in the sorbent core. Thus, by monitoring the applied electrical power, the approximate temperature achieved during a thermal desorption process can be estimated.

For a type III preconcentrator, the heaters are attached to the outside wall of the adsorption tube allowing a quick monitoring of the desorption temperature profile. The heating characteristics of the PCT is measured using a fine-wire type $\mathrm{K}$ thermocouple. The thermal desorption temperature is accurately controlled by a PID temperature controller. A typical temperature profile as a function of time during a heating/cooling cycle is plotted in Figure 2.11. Heat power is subjected to on/off control only, but since the temperature controller responds rapidly to the thermocouple feedback, the temperature overshoot is less than $10{ }^{\circ} \mathrm{C}$ above the set point. The time required for the heater to ramp from room temperature to the peak desorption temperature of $150-220^{\circ} \mathrm{C}$ is less than 15 seconds. 


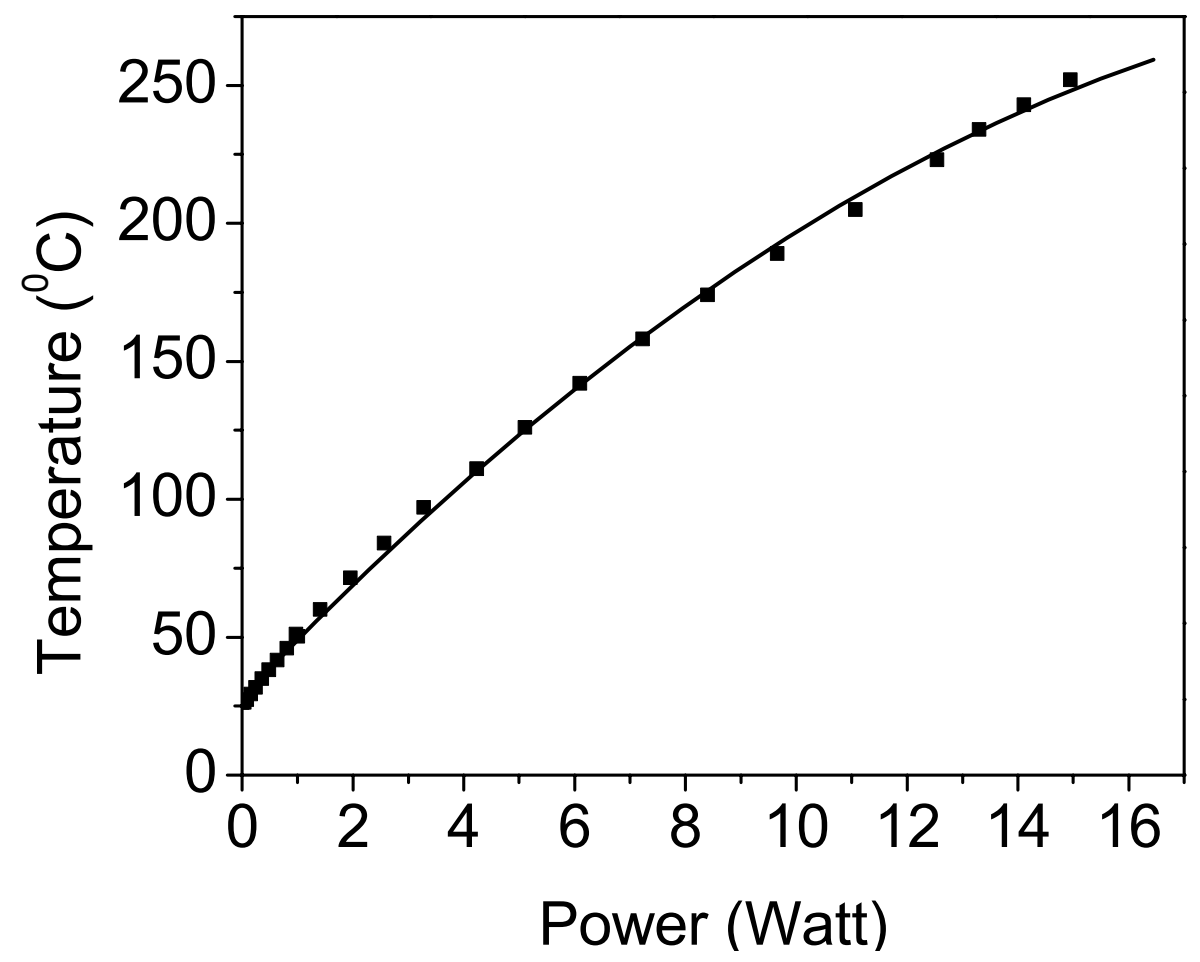

Figure 2.9 The temperature profile of a preconcentration unit (type II) Heater type: Nichrome wire, Heating duration: 15 seconds 


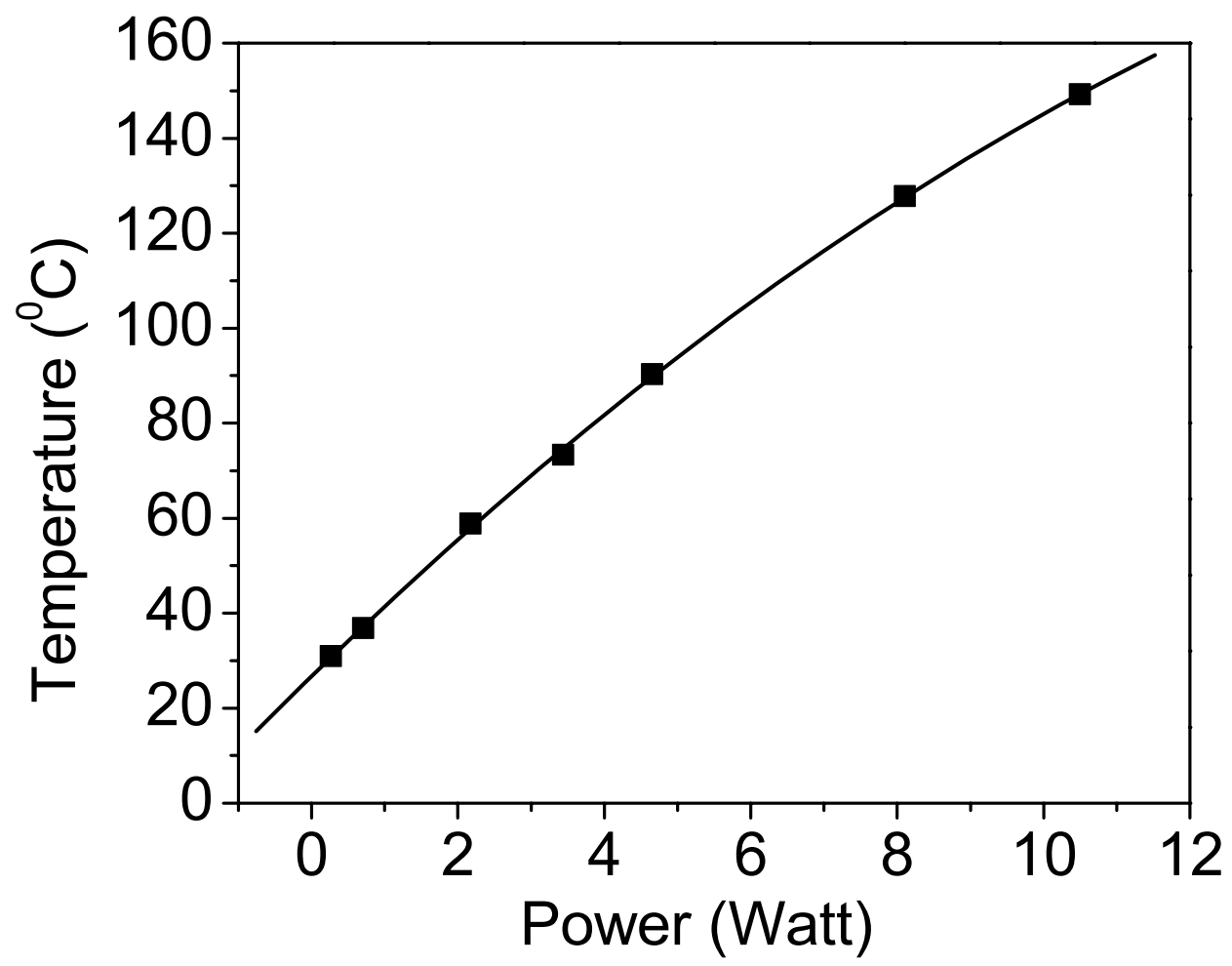

Figure 2.10 The temperature profile of preconcentration unit (type II).

Heater type: Nichrome ribbon; Heating duration: 3 seconds. 


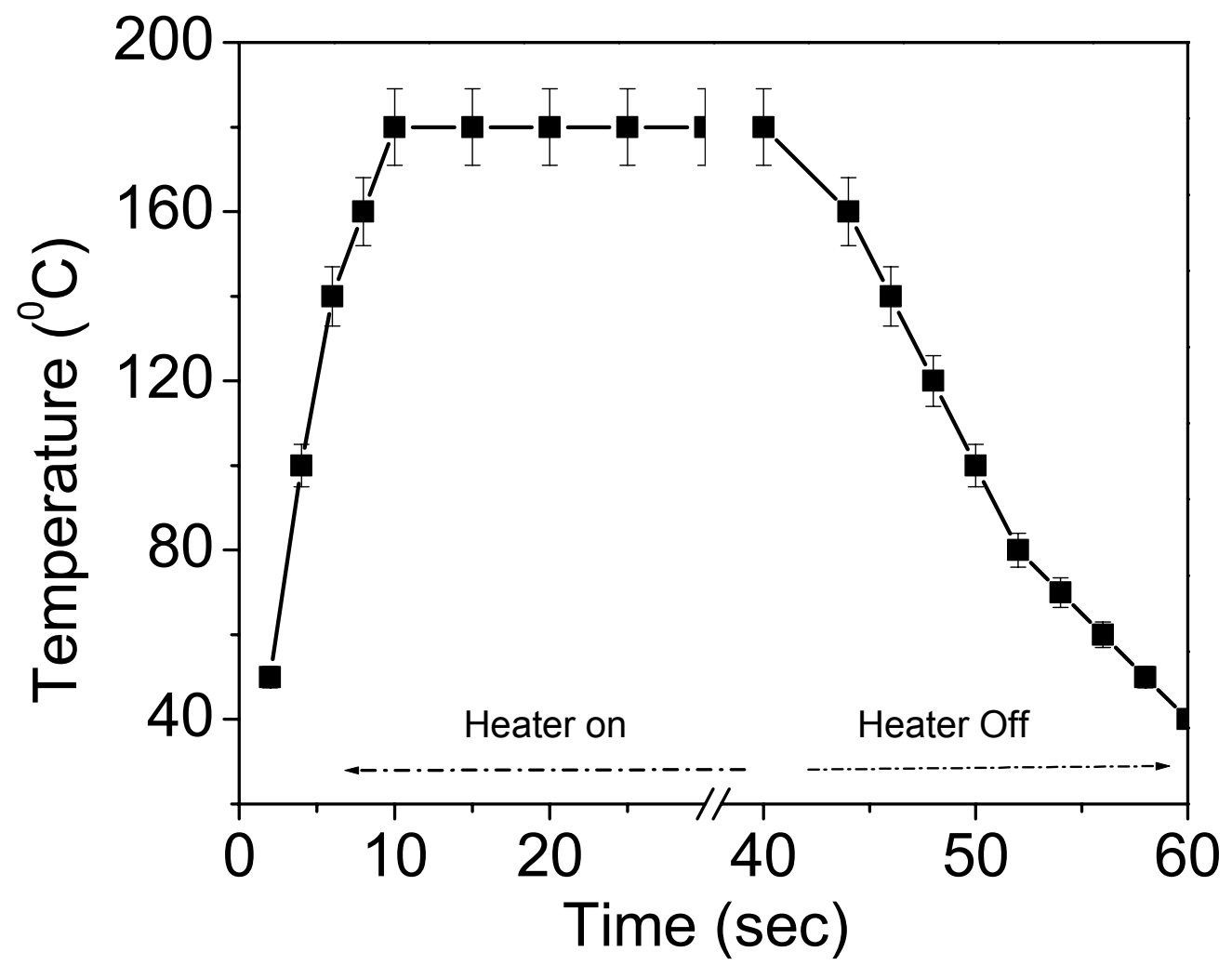

Figure 2.11 The temperature profile of preconcentration unit (type III) in a heating cycle. Heater type: Nichrome wire. (The error line implies a temperature overshoot) 


\subsection{Typical Desorption Profiles}

The preconcentration designs (type II and III) were tested using various toluene vapor concentrations. Direct sample introduction was performed by replacing the adsorption bed with a section of Teflon capillary loop (similar volume compared to PCT units) and measuring the FID signal (relative to blank) after allowing a fixed time for sampling. Several blanks were collected over the course of an experiment and blank subtraction was performed as necessary prior to quantification.

A typical response profile from a PCT system (type II) is illustrated in Figure 2.12 , where the test vapor is toluene at $60 \mathrm{ppm}$ and the Nichrome ribbon is coated with PPY film. The continuous sampling protocol is performed. The vapor stream is introduced to the system for 2 minutes and then the power is applied to the heater. As shown in the figure, the preconcentrator generates a spike signal. The solid line in Figure 2.12 presents the FID response to toluene vapor during the heating and cooling period. Increasing current in the FID results in a more negative signal output to the recording oscilloscope. As expected, the preconcentration with PPY stationary phase provides much higher response than the direct sample introduction (no adsorbent present on the Nichrome ribbon). The direct introduction causes a relatively small response (dashed line) compared to the signals after the preconcentration. The average of FID responses from several blank trials (true zero air baseline)was subtracted from the total response to yield a net response attributed to the test vapor. The spike peak here represents a signal enhancement by a factor of 35 , i.e. the peak signal from the preconcentrator is 35 times that obtained by direct sample 
introduction. This enhancement is defined as the preconcentration factor, which can be used to compare with the preconcentration methods during this experiment.

As shown in Figure 2.12, in this continuous sampling protocol, the analyte is re-adsorbed in the preconcentrator as it is cooled after the desorption. This process changes the FID baseline into a region indicating a "negative concentration", which implies the concentration reaching the FID is smaller than the base concentration in the air stream. As the sample is continuously passing over the adsorbent surface, the FID response increases back to the baseline over a minute. The negative region at the baseline has been reported to be related to the adsorption capacity of the adsorbents. A PCT with adsorbents with a higher adsorption capacity requires longer readsorption times and generates a profile with larger "negative concentration" region [28].

An indirect sampling mode is also used for type II PCT, especially for the preconcentration units with packed adsorbents. One typical example is shown in Figure 2.13, where the preconcentration unit is packed with Tenax GR and the test vapor is toluene at $60 \mathrm{ppm}$. In this process, the dry air stream with the same flow rate as the vapor stream is introduced into the PCT unit to "air-purge" the non-adsorbed vapor analytes after the sampling period. The heater is then actuated after the 5second "air purge". A relative small peak is normally observed which drops back to baseline within a short period. The peak height of this small peak is related to the analyte vapor concentration. Heating the adsorbent releases the sorbed vapor, which then results in the large spike in the FID signal. 
A slightly different indirect sampling mode was used in Type III designs. The vapor sample is pulled through the PCT unit by a small diaphragm pump during the sampling period. The pump is then stopped and the six-port valve is switched, the heater is activated to reach the set-point temperature. in FID signal.. The baseline of FID signal is disturbed and appears to exhibit a "negative concentration" region. The released vapor is back-flushed to the FID by a clean, dry air flow with the same flow rate. A desorption profile for toluene vapor is observed (Figure 2.14). In all cases, the signals for the desorbed vapor appears as a sharp, narrow peak with a slight tailing. The FID signal returns to baseline in less than 1 minute as the PCT unit cools down to room temperature. 


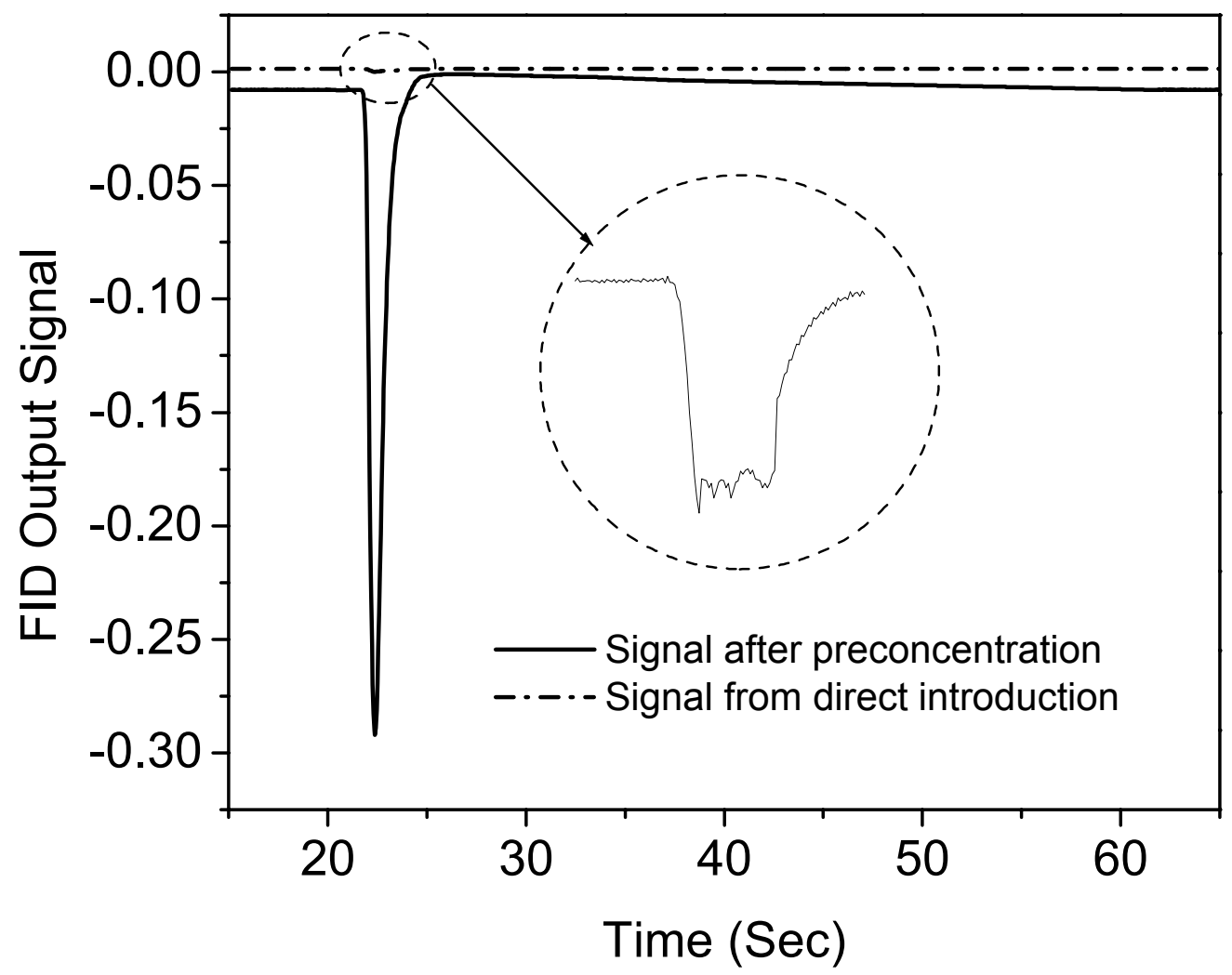

Figure 2.12 A typical thermal desorption profile corresponding to $60 \mathrm{ppm}$ TOL vapor. Preconcentrator type II, Adsorbent: 10 mg PPY coating; sampling time: 2 min; flow rate: $100 \mathrm{ml} / \mathrm{min}$; Heater type: Nichrome ribbon. The signal in the dashed line circle is obtained with 50 expansion of the original scale. 


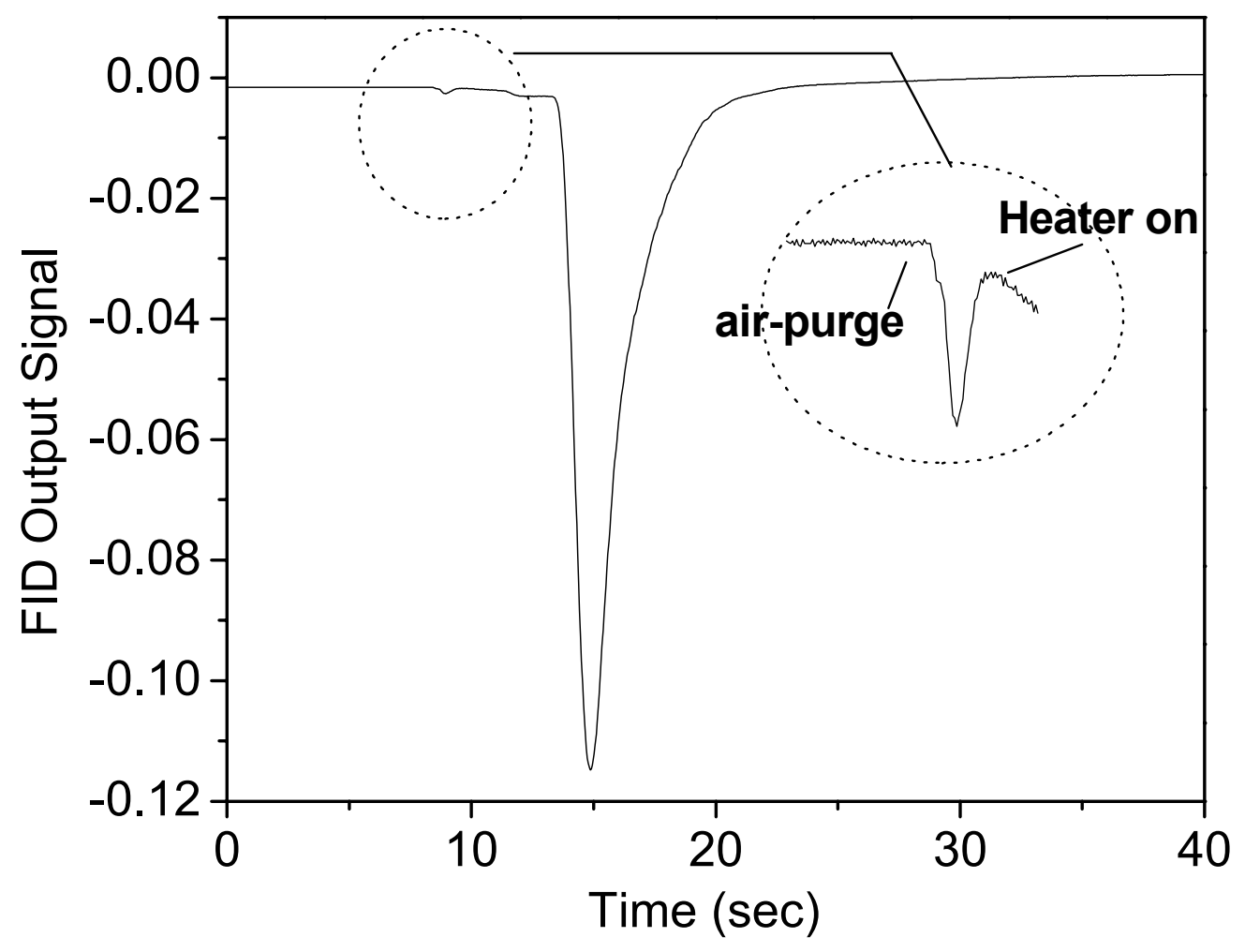

Figure 2.13 Desorption profile of Tenax GR with PCT type II. Applied power: 10 watt; packing amount $30 \mathrm{mg}$; TOL vapor: $60 \mathrm{ppm}$; flow rate: $50 \mathrm{ml} / \mathrm{min}$; heater type: Nichrome wire. Sampling time: 2 min. The signal in the dashed line circle is obtained with 50 expansion of the original scale. 


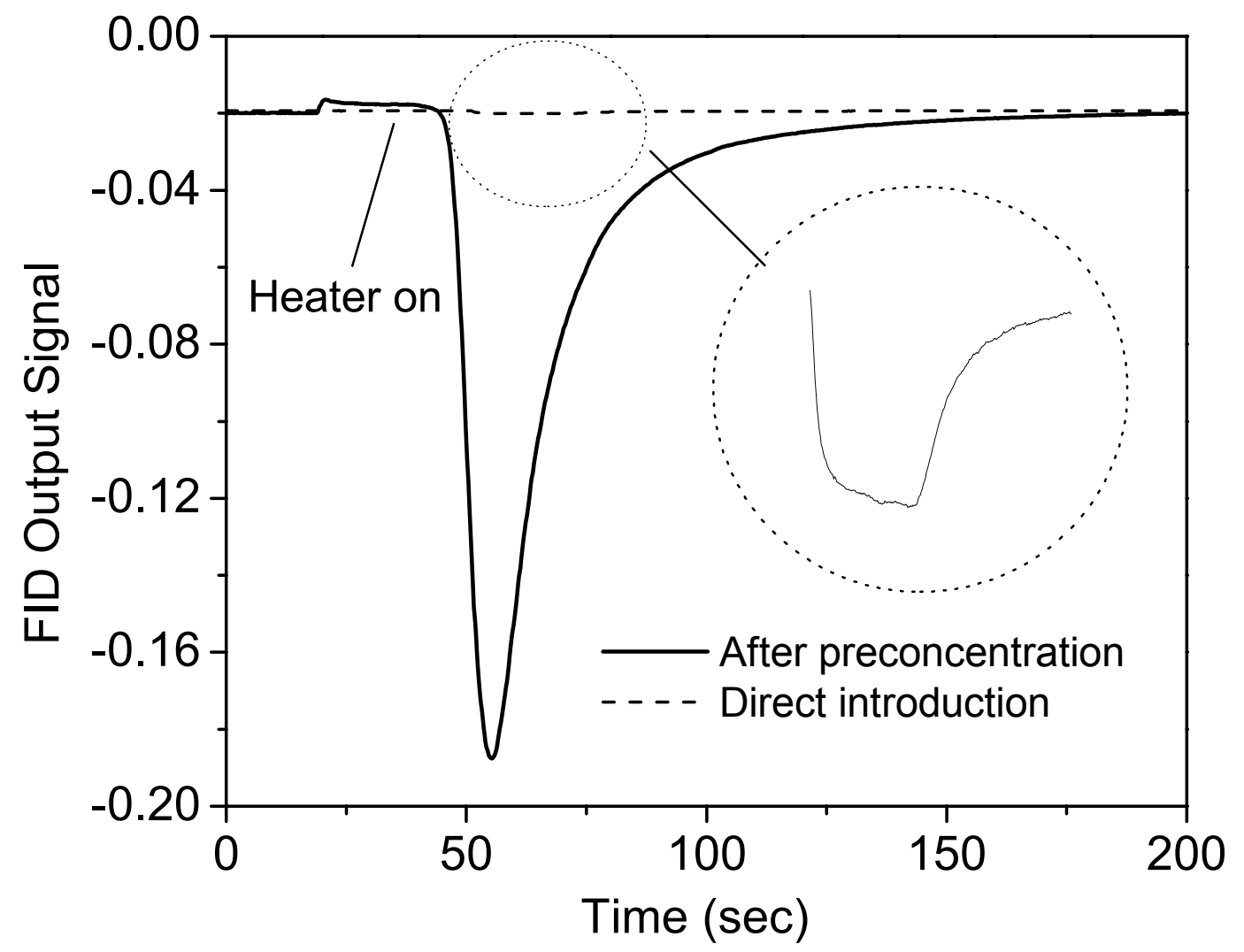

Figure 2.14. Desorption profile of PCT type III. Adsorbent: MIP (HQ); Preconcentration factor 260; TOL vapor: $60 \mathrm{ppm}$, flow rate: $10 \mathrm{ml} / \mathrm{min}$; packing amount $30 \mathrm{mg}$; heater type: Nichrome wire; Sampling time: $5 \mathrm{~min}$; heating time: $30 \mathrm{sec}$. The signal in the dashed line circle is obtained with $\mathbf{5 0}$ expansion of the original scale. 


\subsection{Desorption Characteristics}

\subsubsection{Temperature}

The desorption profile is a function of sorbent temperature. A higher desorption temperature can be obtained either by increasing the voltage across the heater ( increasing applied power) or by a longer resistive heating duration. The resistive heating occurs either at the outside of glass capillary or near the adsorbent surface and the energy is transferred to the sorbent by conduction. This takes a certain amount of time. If enough energy is not supplied to the PCT tube, quantitative desorption does not occur and a small peak results (Figure 2.15 and 2.16). The heater in the different test set-ups requires a power supply of at least $15 \mathrm{~V}$ in order to heat the trap sufficiently quicklyt. Increasing the desorption temperature and the heating rate of the trap should improve the desorption efficiency and sensitivities for all vapors. However a higher desorption temperature would risk decomposition of the porous polymer adsorbents, particular when operated in an air matrix. Some optimization trials of voltage and temperature settings are needed through the experiments. The power settings and the temperature are carefully controlled to avoid the heater to become red-hot.

In both types of preconcentration units (type II and III), higher applied power setting or higher temperature results in relative narrow and sharp peaks as illustrated in figures (Figure 2.15 -2.17). A PCT type II with Nichrome ribbon painted with PPY film exhibits fast thermal desorption behavior with a sharp spike. The Nichrome ribbon heats up very rapidly and transfers heat quickly to the PPY coating. The desorption peak widths (full width at half maximum, FWHM) for toluene vapor are 
less than 1 second at higher desorption temperatures (Table 2.3) and the desorption peak width does not change significantly with the temperature. The peak width at half maximum provides a combined measure of desorption efficiency and sample transport through the system and is used as the performance metric for comparisons between the vapor-adsorbent combinations.

Desorption efficiency is determined by repeating the heating cycle immediately and comparing the magnitude of any residual FID signal to that obtained from the initial sample desorption. The optimum heating temperature is chosen when the sequential response maxima indicate no apparent residual carryover of vapor on the adsorbent in the preconcentrator. Generally, most PCT units are heated up to $180^{\circ} \mathrm{C}$ to achieve the maximum desorption efficiency.

Preconcentration results and desorption bandwidth ( FWHM) data for several powder adsorbents packed in PCT unit type II are summarized in Table 2.4 and 2.5. In general, the FWHM values are larger in packed PCT units than the FWHM values for the Nichrome ribbon coated with PPY. Peak width varies significantly with the desorption temperature. A higher temperature or higher power setting may result in more uniform heating of the sorbent core and therefore a higher preconcentration factor.

Desorption profiles from PCT type II packed with Tenax polymers exhibit broader pulses compared to the same unit packed with PPY powders. One possible cause is that the PPY powder has a relatively low heat capacity and can be heated up very quickly to generate a sharp spike. 

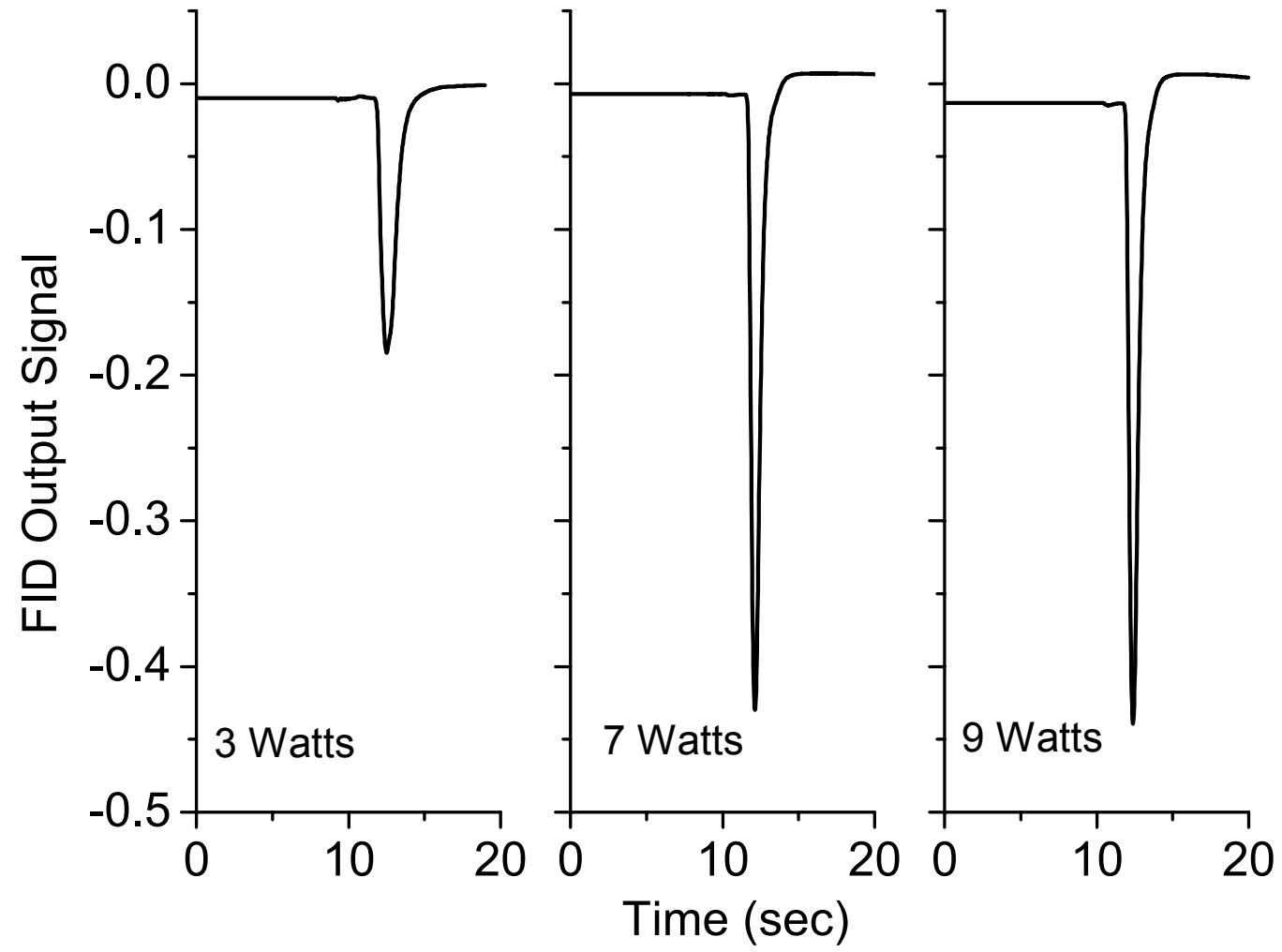

Figure 2.15. Thermal desorption of PPY film GR at different power settings with PCT type II. Coating amount $10 \mathrm{mg}$. Flow rate: $100 \mathrm{ml} / \mathrm{min}$. Vapor concentration: 28 ppm. Sampling time : 5 min. 

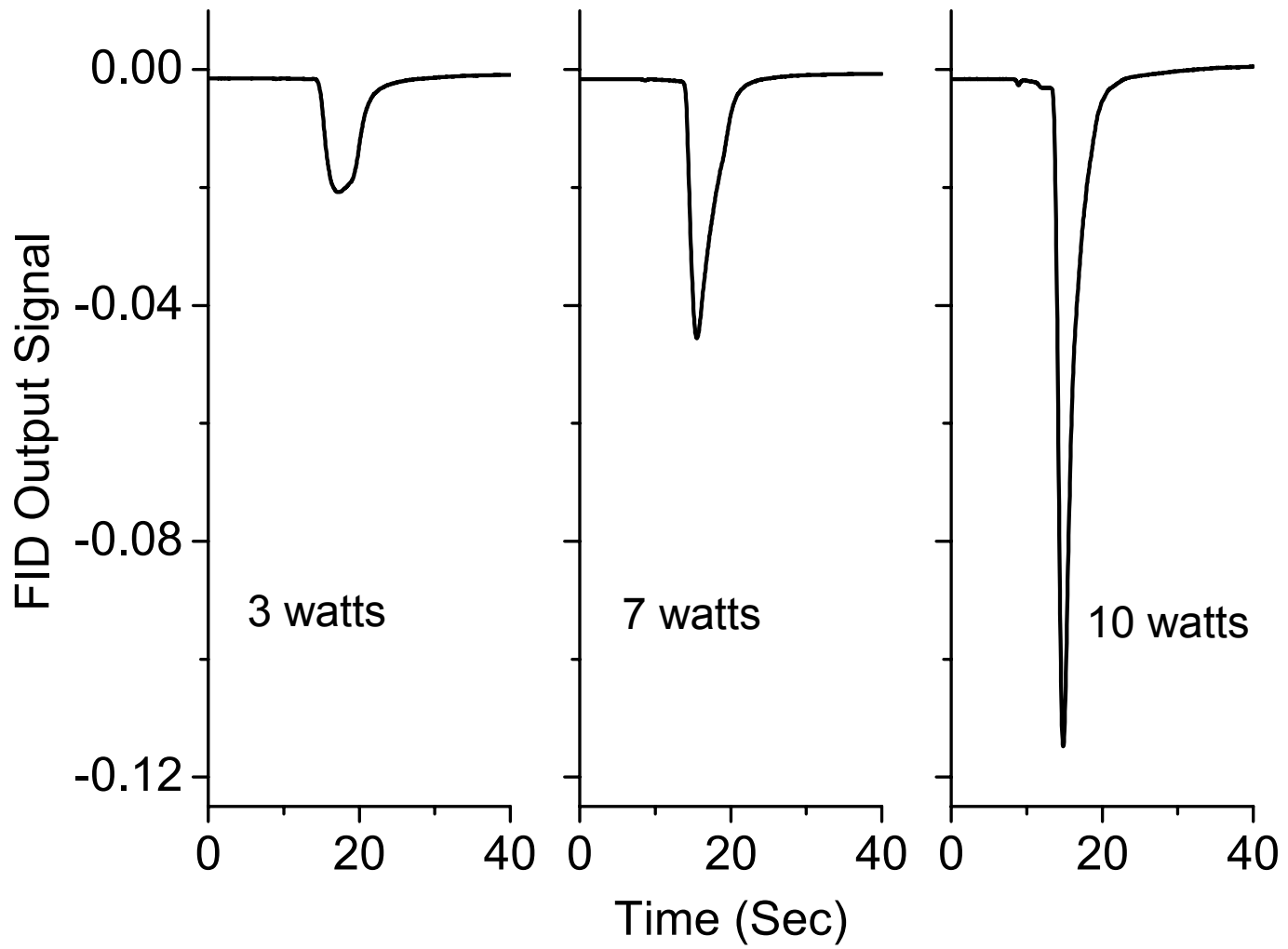

Figure 2.16. Thermal desorption of Tenax GR at different power settings with PCT type II, packing amount $30 \mathrm{mg}$, flow rate: $50 \mathrm{ml} / \mathrm{min}$; vapor concentration: 56ppm, sampling time: $5 \mathrm{~min}$ 


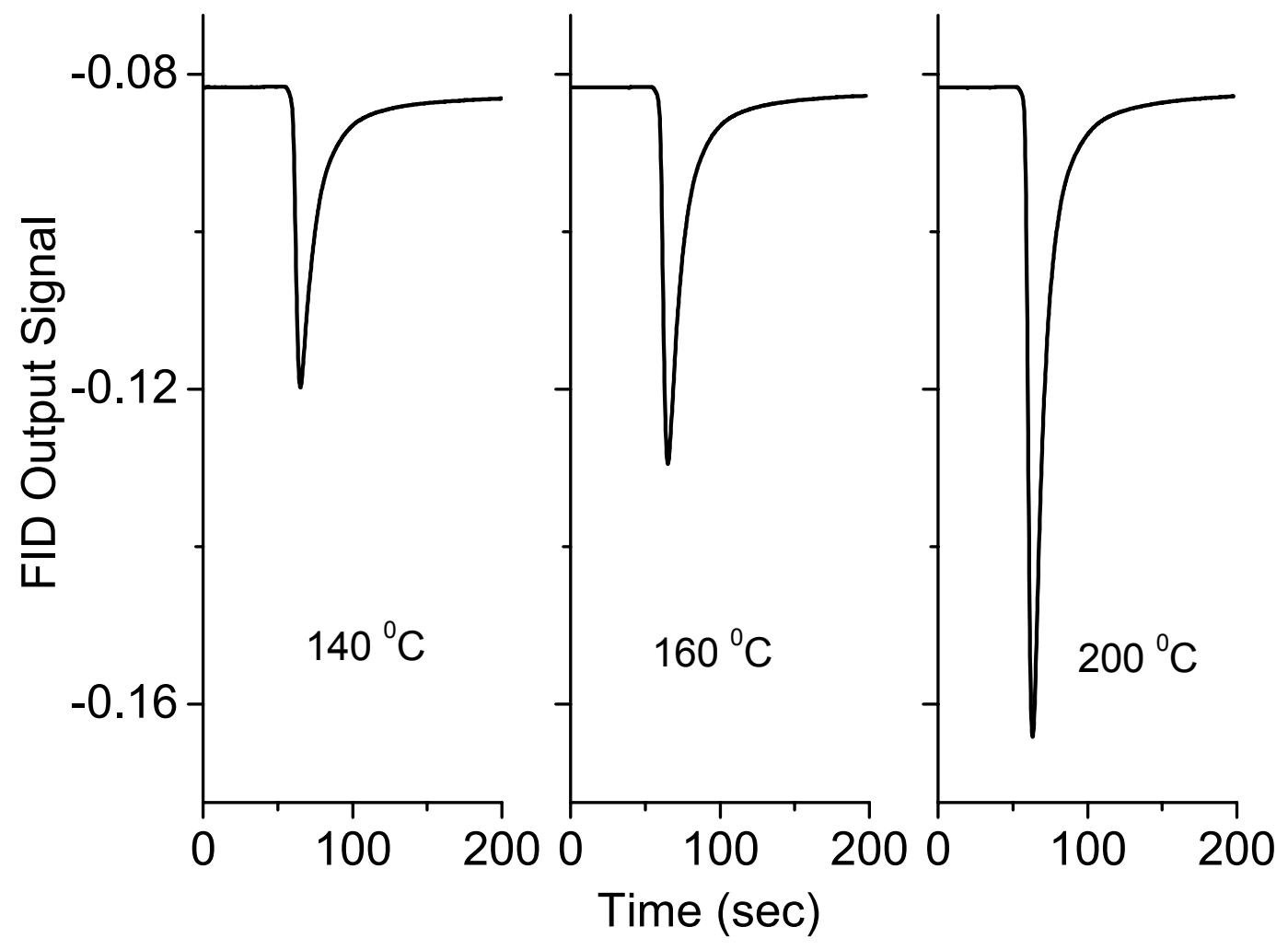

Figure 2.17 Thermal desorption of Tenax GR at different temperatures with PCT type III, packing amount $30 \mathrm{mg}$, flow rate: $10 \mathrm{ml} / \mathrm{min}$; vapor concentration: $14 \mathrm{ppm}$, sampling time: $5 \mathrm{~min}$ 
Table 2.3 Pulses with different applied power

\begin{tabular}{c|c|c|c}
\hline Power (watt) & $\begin{array}{c}\text { FID Pulse height } \\
\text { (V) }\end{array}$ & PF & FWHM (sec) \\
\hline 1.7 & 0.08 & 18 & 0.80 \\
2.6 & 0.115 & 25 & 0.75 \\
5.7 & 0.275 & 60 & 0.62 \\
6.9 & 0.305 & 68 & 0.64 \\
10 & 0.354 & 77 & 0.65 \\
13 & 0.364 & 79 & 0.60 \\
15 & 0.371 & 81 & 0.57 \\
17 & 0.382 & 84 & 0.55 \\
\hline
\end{tabular}

10 mg PPY coating on Nichrome ribbon; heating time 5 seconds; 60 ppm TOL; flow rate: $50 \mathrm{ml} / \mathrm{min}$ 
Table 2.4 Pulses with different applied power using PCT type II packed with PPY powder.

\begin{tabular}{|c|c|c|c|}
\hline Power (watt) & $\begin{array}{c}\text { FID Pulse height } \\
(\mathrm{V})\end{array}$ & PF & FWHM (sec) \\
\hline 5.0 & 0.192 & 28 & 3.84 \\
\hline 6.0 & 0.282 & 40 & 2.40 \\
\hline 8.0 & 0.329 & 50 & 1.64 \\
\hline 10 & 0.469 & 70 & 1.64 \\
\hline 12 & 0.644 & 90 & 1.56 \\
\hline
\end{tabular}

Packing amount: 15 mg; heating time 10 seconds; 60 ppm TOL; flow rate; 50 $\mathrm{ml} / \mathrm{min}$, sampling time: $2 \mathrm{~min}$.

Table 2.5 Thermal Pulses with different applied power using PCT type II packed with Tenax TA.

\begin{tabular}{c|c|c|c}
\hline Power (watt) & $\begin{array}{c}\text { FID Pulse height } \\
\text { (V) }\end{array}$ & PF & FWHM (Sec) \\
\hline 6.0 & 0.178 & 30 & 5.44 \\
8.0 & 0.302 & 50 & 4.08 \\
10 & 0.404 & 70 & 2.74 \\
12 & 0.488 & 80 & 2.16 \\
\hline
\end{tabular}

Packing amount :15 mg, heating time 10 seconds flow rate: $30 \mathrm{ml} / \mathrm{min}$; vapor concentration: $100 \mathrm{ppm}$, sampling time: $2 \mathrm{~min}$ 


\subsubsection{Vapor Concentration}

Calibrations without preconcentration are performed by replacing the adsorption bed with a section of Teflon capillary and measuring the FID signal (relative to blank) after allowing a similar time interval for sampling. With no preconcentrator on line, the FID responds linearly with respect to TOL vapor at different concentrations ( Figure 2.18 and 2.19).

A series of preconcentration tests are carried out to investigate the performance of PCT designs of type II and III at various concentrations based on different stationary phases. Two to four replicates are performed at each concentration. Preconcentration factors are roughly calculated by comparing the preconcentrated signals to those from calibration curves, respectively. The sampling operation with PCT type II is achieved by a vapor stream directly from KinTek vapor system. The tested vapor concentrations are at relatively high ppm level. The PCT type III is tested with rather lo vapor concentrations (low ppm ranges) pumped from Tedlar bags. FID responses are taken as the height of the peak relative to the baseline. The baseline is determined 5 min after turning off the heater on PCT during the desorption mode.

Some preconcentration experiment results from PCT type II are summarized in Table 2.6. Type II PCTs are tested at the toluene concentration range from 30 ppm to a few hundred ppm. The obtained preconcentrated signals are at least 10 times greater than signals from direct sample introduction. A relatively high preconcentration factor around 100 is obtained with packed PPY powders. The FID signals is nearly independent of the vapor concentration. As the concentration 
increases, the preconcentration factor achieved decreases. Changes in peak width are minimal, so peak areas as well as peak heights are not linear with vapor concentrations. This suggests that all possible adsorption sites on the adsorbents are occupied (saturated) even at the lower vapor concentrations. This conclusion is different from the breakthrough studies ( In the latter studies, the amount of adsorbed material is nearly linear with the vapor concentration as shown in Figure 1.41). We have no explanation to this disagreement. This issue should be further investigated in the future work.

When using the type III design for vapor preconcentration (Table 2.7), the peak half widths are larger because the heater is located outside of the adsorption tube, and possibly because a temperature controller is used to set the heating rate. Despite the larger peak width, rather impressive preconcentration factor values are obtained at the lower concentration levels. Again, we do not have an explanation for decreasing preconcentration factor values as vapor concentration increases. Henry's Law does not appear to work. However, molecularly imprinted polymers (MIPs) are clearly superior to other adsorbents used in the experiments. Preconcentration factors on the order of 1000 are achieved by using the BIP(HQ) polymer as adsorbents in type III design at low ppm concentration ranges .

For low concentrations of analyte, where the adsorption isotherm is expected to be linear, the preconcentration factor should be independent of the analyte concentration. The factors influencing the preconcentration factor are the activity and area of the collection surface, the ambient temperature and the desorption volume [29]. With a fixed system, the major parameter affecting the preconcentration factor is 
the desorption pulse volume. The desorption pulse volume is controlled by the purge gas flow rate and the rate of heating. Consequently, the preconcentration factor is optimized by adjustment of the flow of dry air passed through the unit and into the FID detector during the desorption.

Peak widths (FWHM) of desorbed samples ranges from hundreds of milliseconds with type II designs to a few seconds with type III designs. The FWHM of the response profiles reflects the desorption rate during the preconcentration process and depends on the operation conditions. Generally, lower concentrations yield higher FWHMs (Figure 2.20).

The profiles of the desorption peaks are plotted in Figure2.21and 2.22 as a function of flow rate. It can be seen that the desorption peak height increases sharply with the increasing flow rates. At a low flow rate, the desorbed analyte pulse moves slowly. The PCT cools down significantly before the desorbed vapor has left the adsorbent bed and results in a broad desorption peak. At high flow rates, the pulse escapes the PCT before it cooled significantly. As flow rate increases, back pressure is built up and becomes a big issue to affect PCT performance. Since we use glass capillary as adsorption tube in the preconcentration design, the low desorption flow rate of $10 \mathrm{ml} / \mathrm{min}$ is adopted in the protocols. 
Longer exposure time results in increased sample volume and is explored as a means of increasing sensitivity. Thermal desorption results of a PPY film with varying exposure time are shown in Figure 2.23. Short exposure times (a few seconds) results a small and broad desorption peak for PPY film. As exposure time increase, the FID signal increases. The FID peak signals change only slightly when the exposure time is extended to more than 1 minute. Similar results are observed in Figure 2.24, where PCT type II packed with PPY powder is investigated. The FID peak signals vary significantly with the exposure times. A sampling time of 5 minutes is sufficient to achieve uniform desorption profiles. Information of peak height vs exposure time are always obtained to optimize the sample collection time needed. 


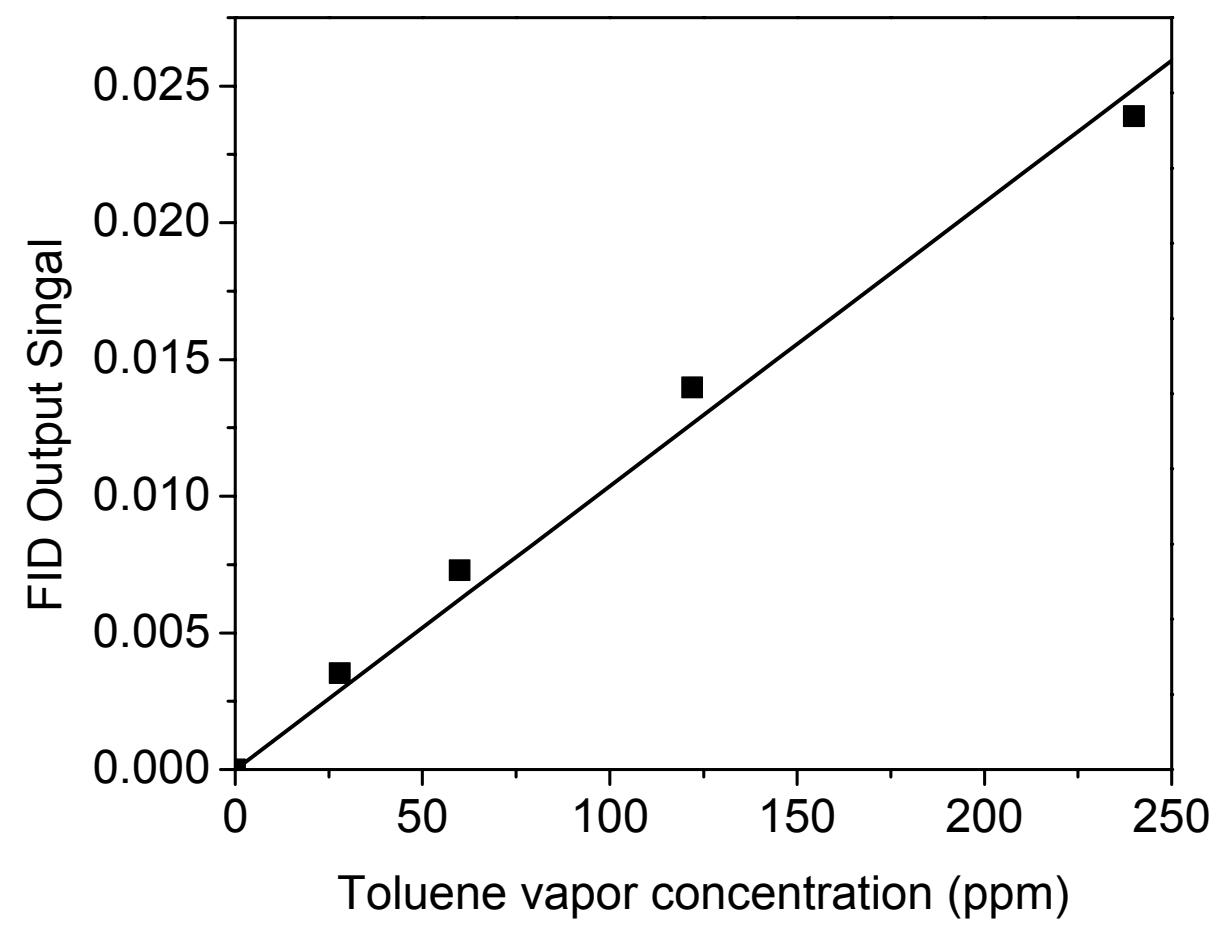

Figure 2.18 Calibration curve used in PCT type II. $y=1.03 \times 10^{-4} x, r^{2}=0.992$; air flow rate: $50 \mathrm{ml} / \mathrm{min}$; 


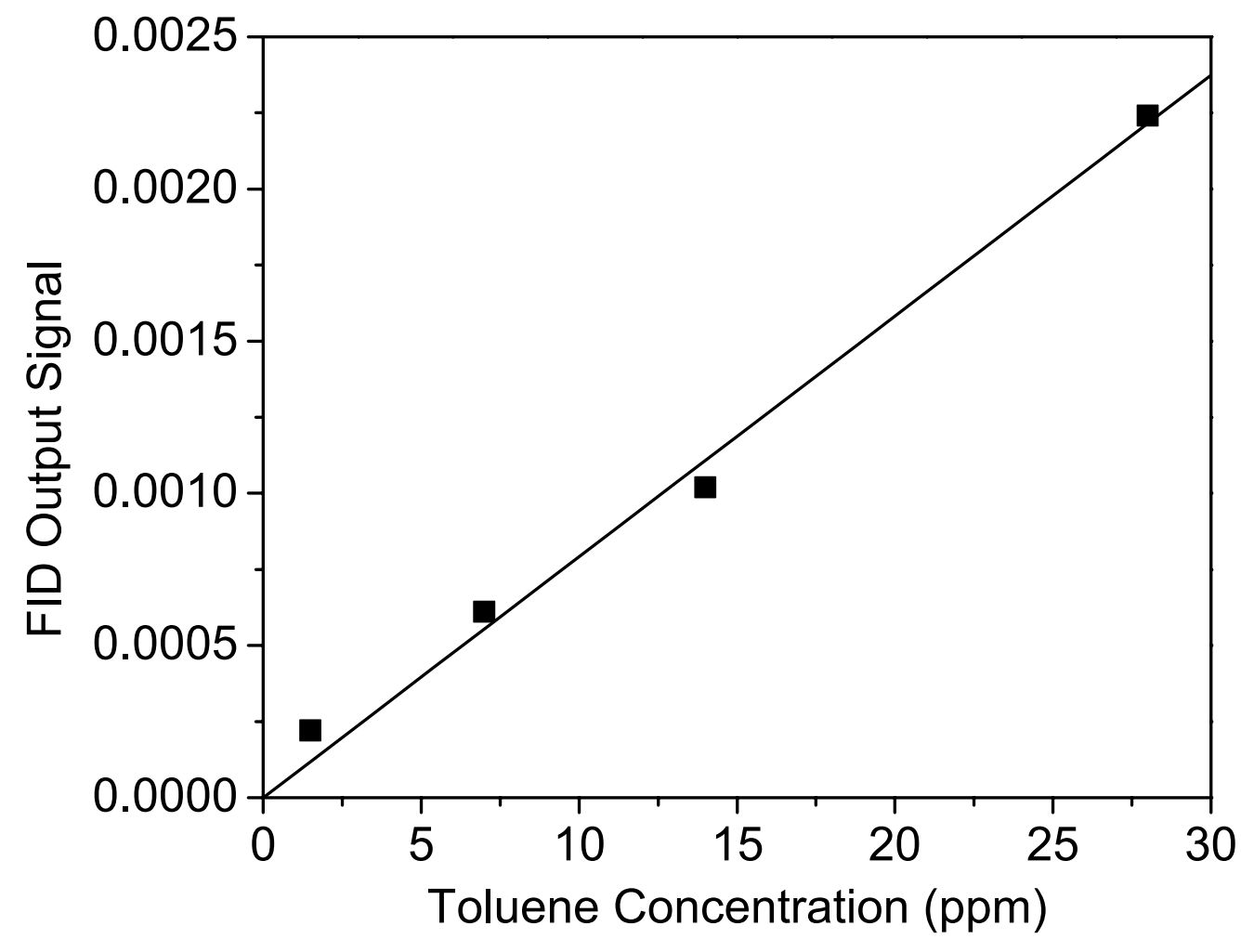

Figure 2.19. Calibration curve for toluene vapor used in PCT type III. $y=7.9 \times 10^{-5} x ; r^{2}=0.994 ;$ air flow rate: $10 \mathrm{ml} / \mathrm{min}$; 
Table 2.6 Data of Preconcentration Factor and FWHM from Type II PCT

a. PPY film

\begin{tabular}{c|c|c|c}
\hline $\begin{array}{c}\text { Concentration } \\
(\mathrm{ppm})\end{array}$ & $\begin{array}{c}\text { FID pulse height } \\
(\mathrm{V})\end{array}$ & FWHM (sec) & PF \\
\hline 30 & $0.240 \pm 0.002$ & $0.82 \pm 0.01$ & 65 \\
40 & $0.251 \pm 0.003$ & $0.8 \pm 0.01$ & 50 \\
60 & $0.263 \pm 0.002$ & $0.82 \pm 0.01$ & 40 \\
90 & $0.270 \pm 0.001$ & $0.88 \pm 0.02$ & 30 \\
120 & $0.276 \pm 0.004$ & $0.88 \pm 0.01$ & 25 \\
180 & $0.281 \pm 0.003$ & $0.86 \pm 0.02$ & 20 \\
240 & $0.284 \pm 0.004$ & $0.88 \pm 0.02$ & 15 \\
\hline
\end{tabular}

$10 \mathrm{mg}$ coating, flow rate: $100 \mathrm{ml} / \mathrm{min}$, sampling time: $2 \mathrm{~min}$

b. PPY powder : $30 \mathrm{mg}$

\begin{tabular}{c|c|c|c}
\hline $\begin{array}{c}\text { Concentration } \\
(\mathrm{ppm})\end{array}$ & $\begin{array}{c}\text { FID pulse height } \\
(\mathrm{V})\end{array}$ & $\begin{array}{c}\text { FWHM } \\
(\mathrm{Sec})\end{array}$ & PF \\
\hline 30 & $0.291 \pm 0.001$ & $1.36 \pm 0.02$ & 95 \\
60 & $0.294 \pm 0.001$ & $1.72 \pm 0.02$ & 50 \\
120 & $0.300 \pm 0.002$ & $1.12 \pm 0.02$ & 25 \\
240 & $0.310 \pm 0.002$ & $0.96 \pm 0.01$ & 13 \\
\hline
\end{tabular}

Packing amount: $30 \mathrm{mg}$, flow rate: $50 \mathrm{ml} / \mathrm{min}$, sampling time : $5 \mathrm{~min}$. 
C. Tenax TA

\begin{tabular}{c|c|c|c}
\hline $\begin{array}{c}\text { Concentration } \\
(\mathrm{ppm})\end{array}$ & $\begin{array}{c}\text { FID pulse height } \\
(\mathrm{V})\end{array}$ & $\begin{array}{c}\text { FWHM } \\
(\mathrm{Sec})\end{array}$ & PF \\
\hline 30 & $0.260 \pm 0.001$ & $2.80 \pm 0.01$ & 80 \\
60 & $0.322 \pm 0.001$ & $2.72 \pm 0.02$ & 54 \\
120 & $0.334 \pm 0.002$ & $2.64 \pm 0.02$ & 28 \\
240 & $0.490 \pm 0.002$ & $2.24 \pm 0.02$ & 20 \\
\hline
\end{tabular}

Packing amount: $30 \mathrm{mg}$, flow rate: $50 \mathrm{ml} / \mathrm{min}$, sampling time : $5 \mathrm{~min}$.

d. Tenax GR

\begin{tabular}{c|c|c|c}
\hline $\begin{array}{c}\text { Concentration } \\
(\mathrm{ppm})\end{array}$ & $\begin{array}{c}\text { FID pulse height } \\
(\mathrm{V})\end{array}$ & $\begin{array}{c}\text { FWHM } \\
(\mathrm{Sec})\end{array}$ & PF \\
\hline 30 & $0.167 \pm 0.001$ & $1.92 \pm 0.01$ & 50 \\
60 & $0.238 \pm 0.001$ & $1.86 \pm 0.02$ & 40 \\
120 & $0.338 \pm 0.002$ & $1.84 \pm 0.02$ & 27 \\
240 & $0.592 \pm 0.003$ & $1.82 \pm 0.02$ & 24 \\
\hline
\end{tabular}

Packing amount: $\mathbf{3 0} \mathrm{mg}$, flow rate: $50 \mathrm{ml} / \mathrm{min}$, sampling time: $5 \mathrm{~min}$ 
Table 2.7 Data of Preconcentration Factor and FWHM from Type III PCT

a. Tenax TA (Type III): $10 \mathrm{mg}$; flow rate: $10 \mathrm{ml} / \mathrm{min}$, sampling time: $5 \mathrm{~min}$

\begin{tabular}{|c|c|c|c|}
\hline Concentration (ppm) & FID pulse height (V) & FWHM(Sec) & PF \\
\hline 0.7 & $0.0197 \pm 0.0001$ & $5.8 \pm 0.2$ & 400 \\
\hline 1.5 & $0.0346 \pm 0.0001$ & $5.2 \pm 0.2$ & 300 \\
\hline 7 & $0.0521 \pm 0.0002$ & $5.4 \pm 0.2$ & 100 \\
\hline 14 & $0.0667 \pm 0.0003$ & $5.4 \pm 0.1$ & 65 \\
\hline 28 & $0.102 \pm 0.002$ & $4.8 \pm 0.1$ & 45 \\
\hline
\end{tabular}

b. Tenax GR (type III): $10 \mathrm{mg}$; flow rate: $10 \mathrm{ml} / \mathrm{min}$, sampling time : $5 \mathrm{~min}$

\begin{tabular}{|c|c|c|c|}
\hline Concentration (ppm) & FID pulse height (V) & FWHM (Sec) & PF \\
\hline 0.7 & $0.0121 \pm 0.001$ & $5.8 \pm 0.3$ & 250 \\
\hline 1.5 & $0.0120 \pm 0.001$ & $5.3 \pm 0.2$ & 100 \\
\hline 7 & $0.0492 \pm 0.002$ & $4.8 \pm 0.1$ & 95 \\
\hline 14 & $0.0774 \pm 0.002$ & $3.4 \pm 0.2$ & 75 \\
\hline 28 & $0.110 \pm 0.003$ & $2.6 \pm 0.2$ & 48 \\
\hline
\end{tabular}

C. MIP (Type III): $10 \mathrm{mg}$; flow rate: $10 \mathrm{ml} / \mathrm{min}$; sampling time: $5 \mathrm{~min}$

\begin{tabular}{|c|c|c|c|}
\hline Concentration (ppm) & FID pulse height (V) & FWHM (Sec) & PF \\
\hline 0.7 & $0.112 \pm 0.001$ & $6.4 \pm 0.3$ & 2250 \\
\hline 1.5 & $0.126 \pm 0.001$ & $5.8 \pm 0.2$ & 1100 \\
\hline 7 & $0.362 \pm 0.002$ & $3.8 \pm 0.2$ & 700 \\
\hline 14 & $0.694 \pm 0.002$ & $3.4 \pm 0.2$ & 670 \\
\hline 28 & $0.876 \pm 0.003$ & $3.4 \pm 0.2$ & 400 \\
\hline
\end{tabular}




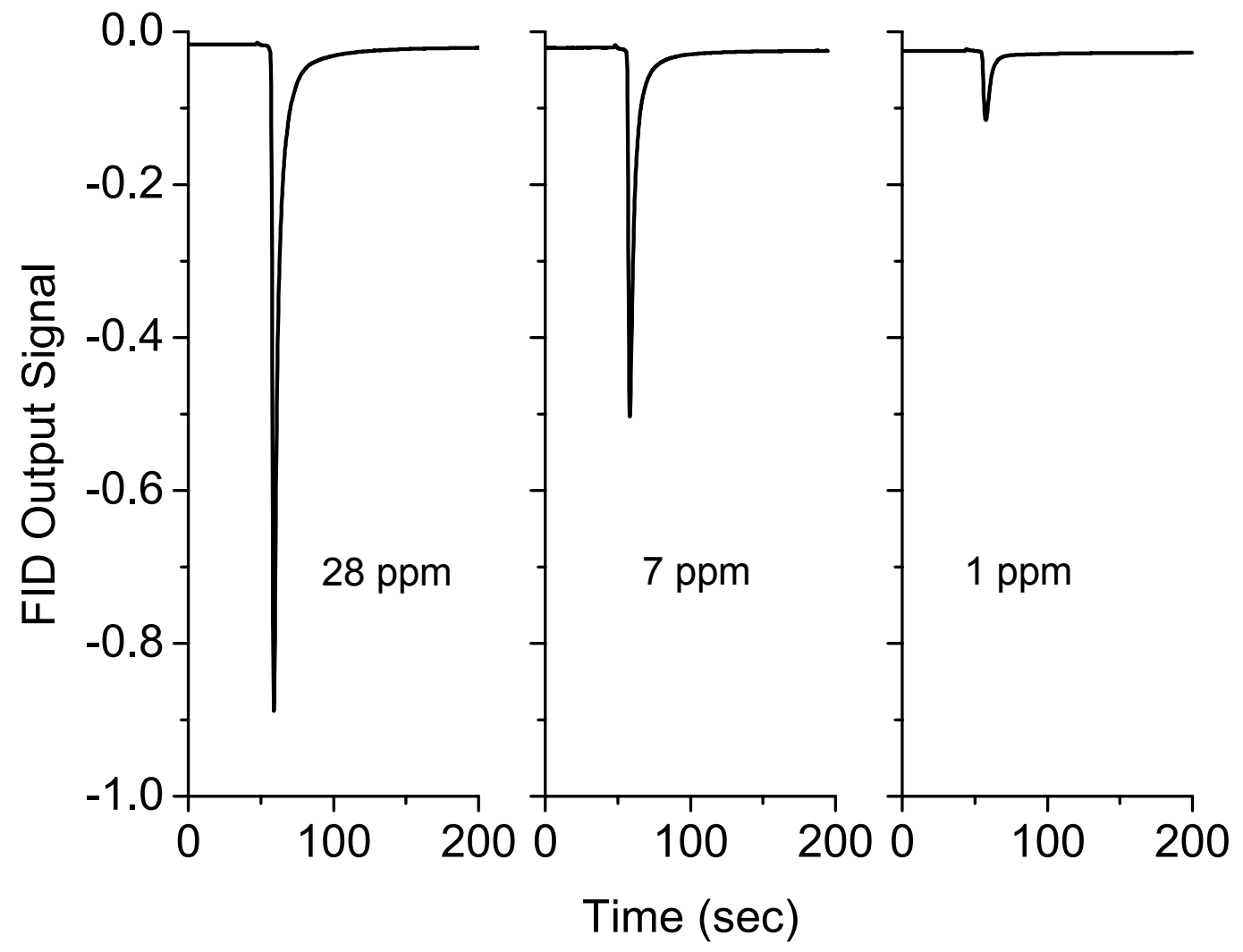

Figure 2.20. MIP thermal desorption profiles with respect to different vapor concentrations. Preconcentrator type III; Flow rate: $10 \mathrm{ml} / \mathrm{min}$.

FWHM values (left to right): $3.4 \mathrm{Sec} ; 3.8 \mathrm{sec} ; 6.0 \mathrm{sec}$. 


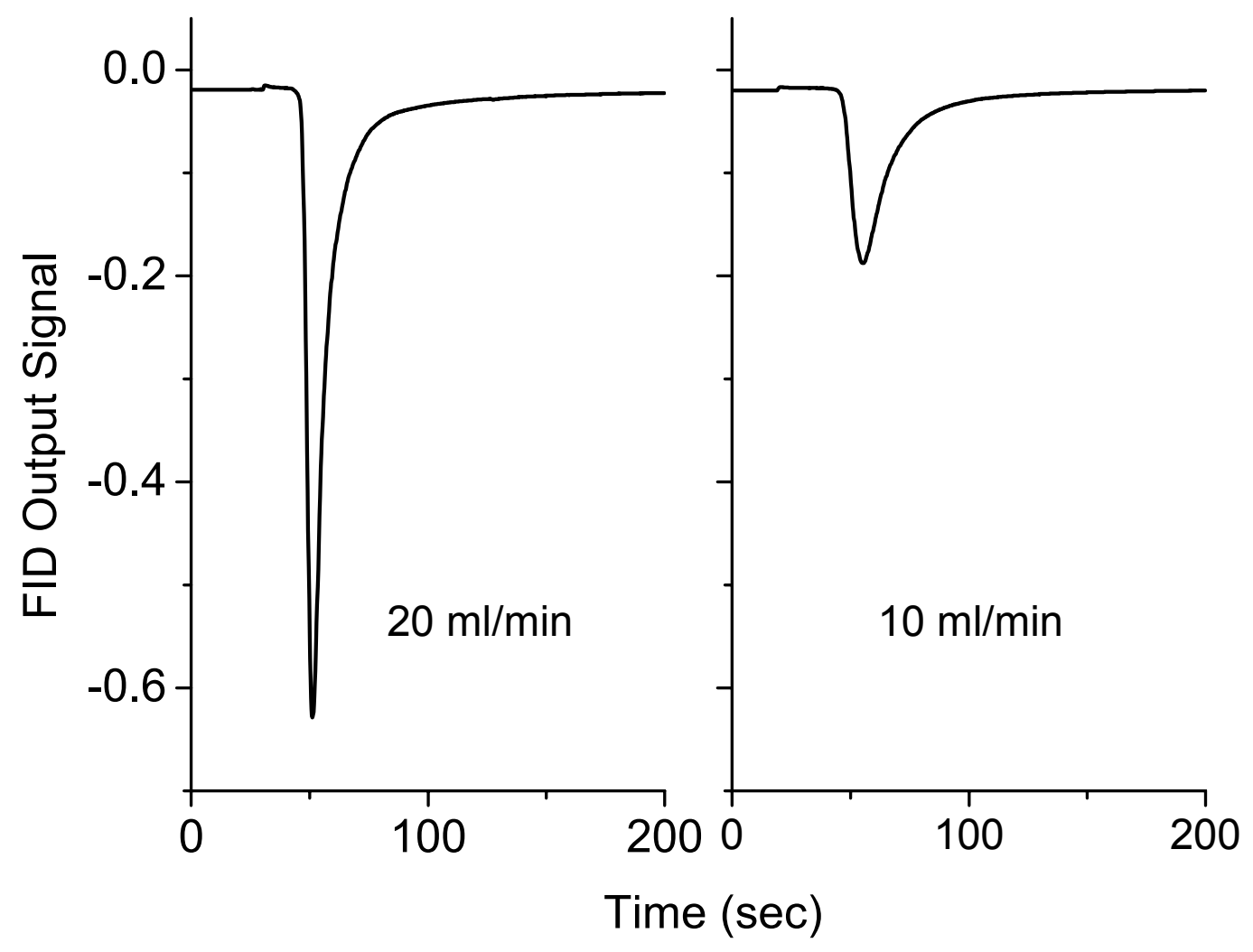

Figure 2.21. Thermal desorption profiles of Tenax GR under different desorption flow rates. Preconcentrator type III. TOL vapor concentration: 60 ppm. FWHM values (left to right): $3.3 \mathrm{sec} ; 5.6 \mathrm{sec}$. 


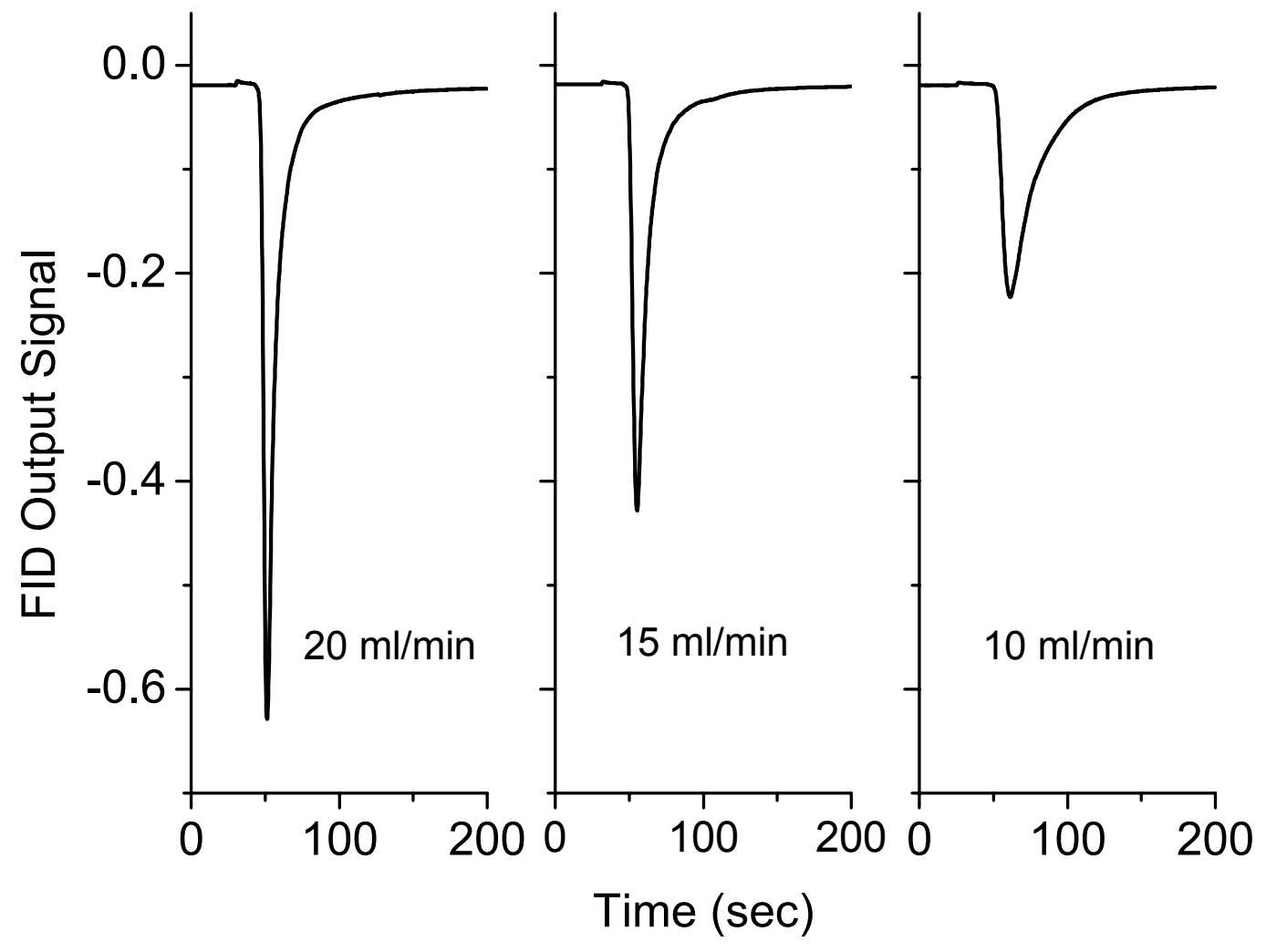

Figure 2.22 . Thermal desorption profiles of MIP under different desorption flow rates. Preconcentrator type III. Vapor Concentration. TOL vapor concentration 5 ppm. FWHM values (left to right): $3.3 \mathrm{sec} ; 3.9 \mathrm{sec} ; 5.6 \mathrm{sec}$. 


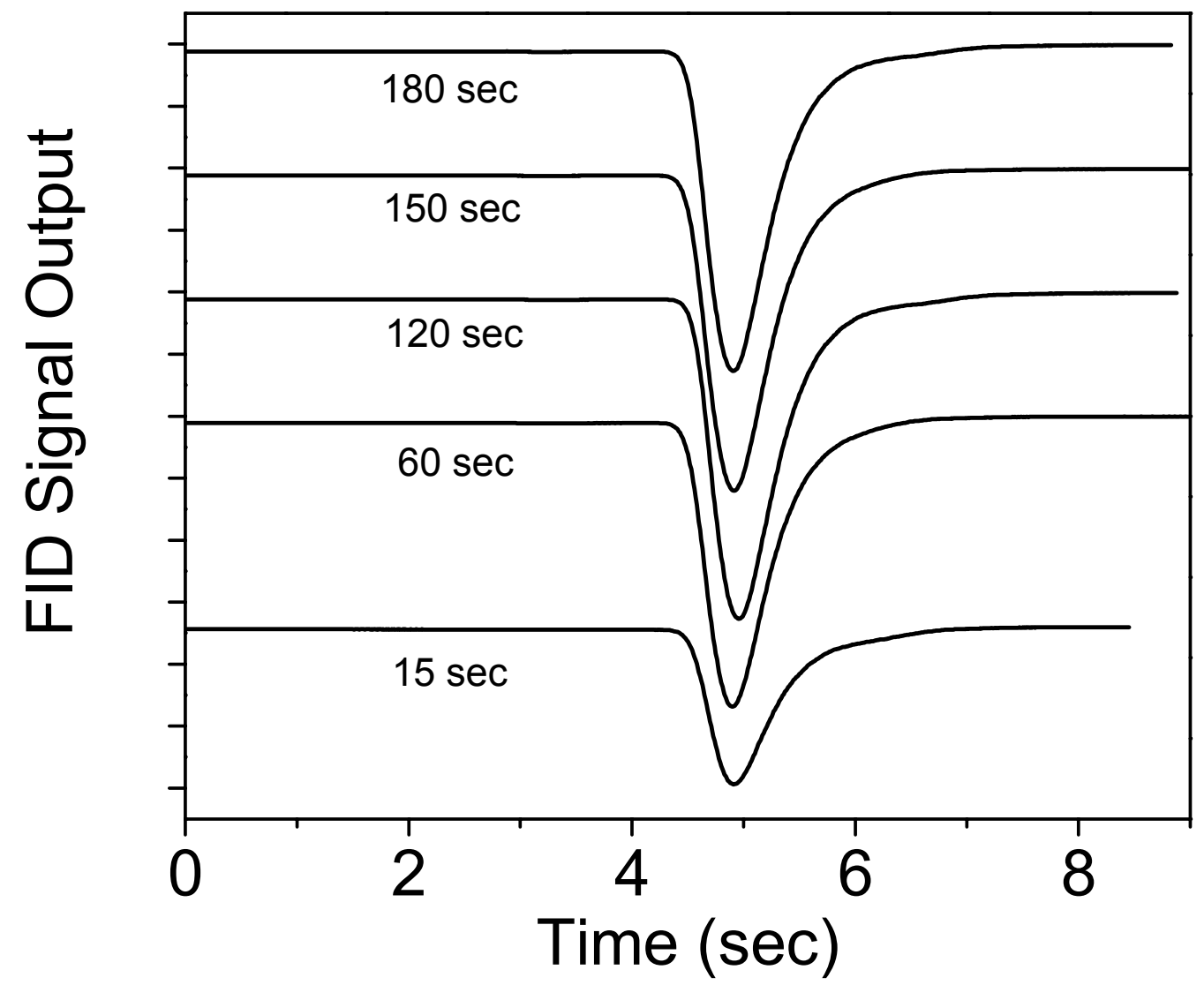

Figure 2.23. Desorption profiles of PPY film with different sample collecting time. Type II preconcentrator. FWHM values (top to bottom): $0.52 \mathrm{sec} ; 0.56 \mathrm{sec} ; 0.64 \mathrm{sec} ; 0.78 \mathrm{Sec}$; $0.84 \mathrm{Sec}$. 


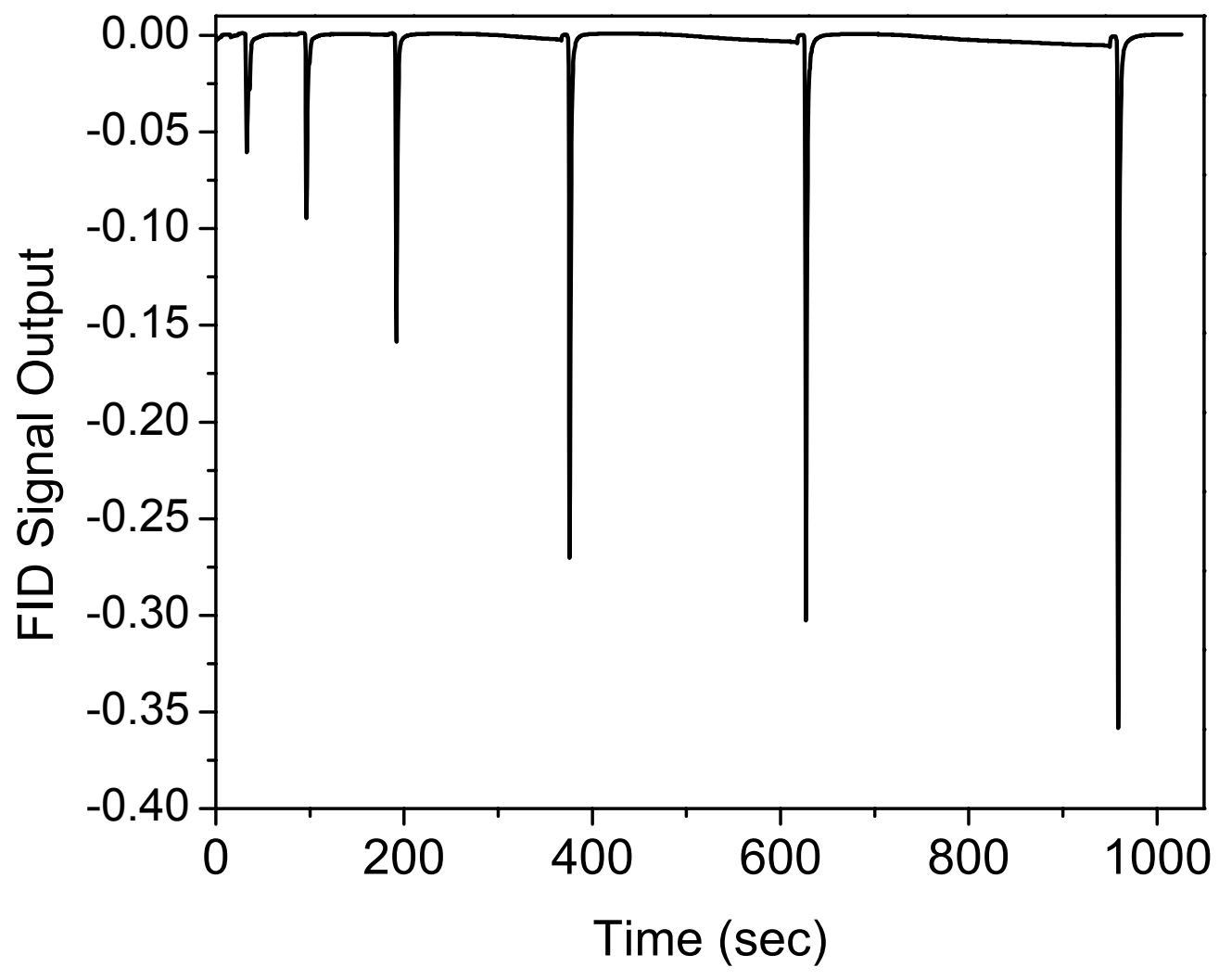

Figure 2.24. Desorption profiles of PPY powder (Bed packing in Type II) with different sample collecting time. Exposure times for different pulses are: $60 \mathrm{sec}, 120 \mathrm{sec}$, $180 \mathrm{sec}, 240 \mathrm{sec}, 360 \mathrm{sec}$. 


\subsection{Adsorbent selection}

The ideal sorbent for PCT in this project would be one that has high selectivity for the volatile species of interest, and at the same time has a large sampling capacity. There is still no single commercial sorbent that satisfy the above mentioned criteria of selective trapping of the small volatile molecules.

Results from QCM studies and breakthrough studies (Chapter I) suggest that synthesized MIP materials possess larger adsorption capacity and higher selectivity toward flat aromatic compounds compared to traditional sorbents Tenax TA and GR. The advantage of using MIPs for PCT packing is also demonstrated by the thermal desorption profiles. Figure 2.25 shows the preconcentrated peaks for heptanes obtained from Tenax GR and a MIP material. Both adsorbents give desorption peaks with similar shapes for heptanes. A slightly larger peak observed for the MIP is not surprising, given its relatively higher adsorption capacity for heptanes. Different desorption profiles toward toluene vapor are also compared for Tenax TA, GR and MIP materials in Figure 2.26. Again, among these adsorbents, MIP achieves a more efficient TOL preconcentration. Other experiment results are already presented in Table 2.6. The fairly high preconcentration factor on the order of 1000 is achieved at lower concentration level. All these results suggest that MIP materials are promising for PCT adsorbents. 


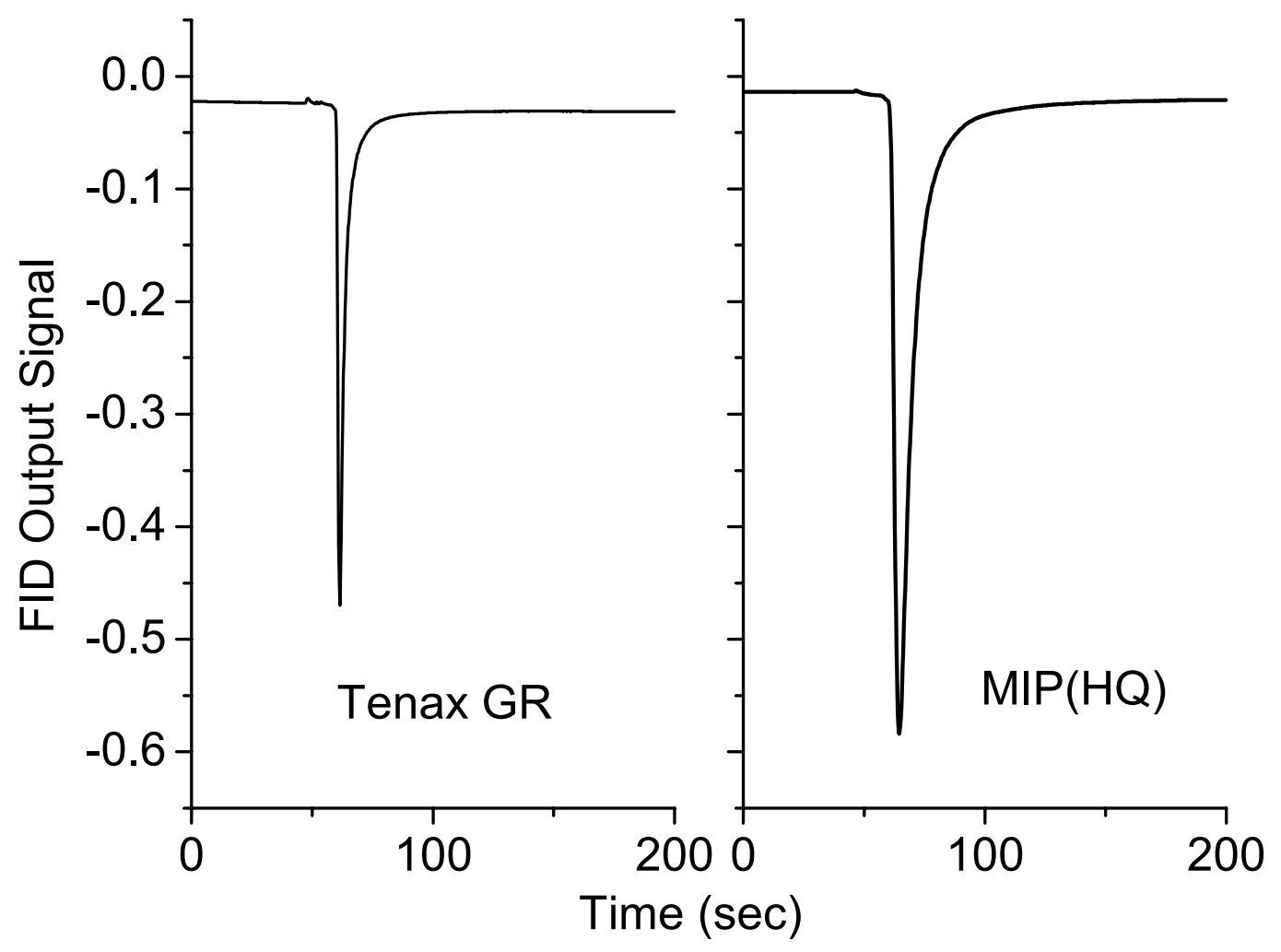

Figure 2.25. Thermal desorption toward Heptanes. Vapor concentration: 80 ppm; $10 \mathrm{mg}$ preconcentrator type $\mathrm{III}$; flow rate $20 \mathrm{ml} / \mathrm{min}$; packing amount : $10 \mathrm{mg}$. 


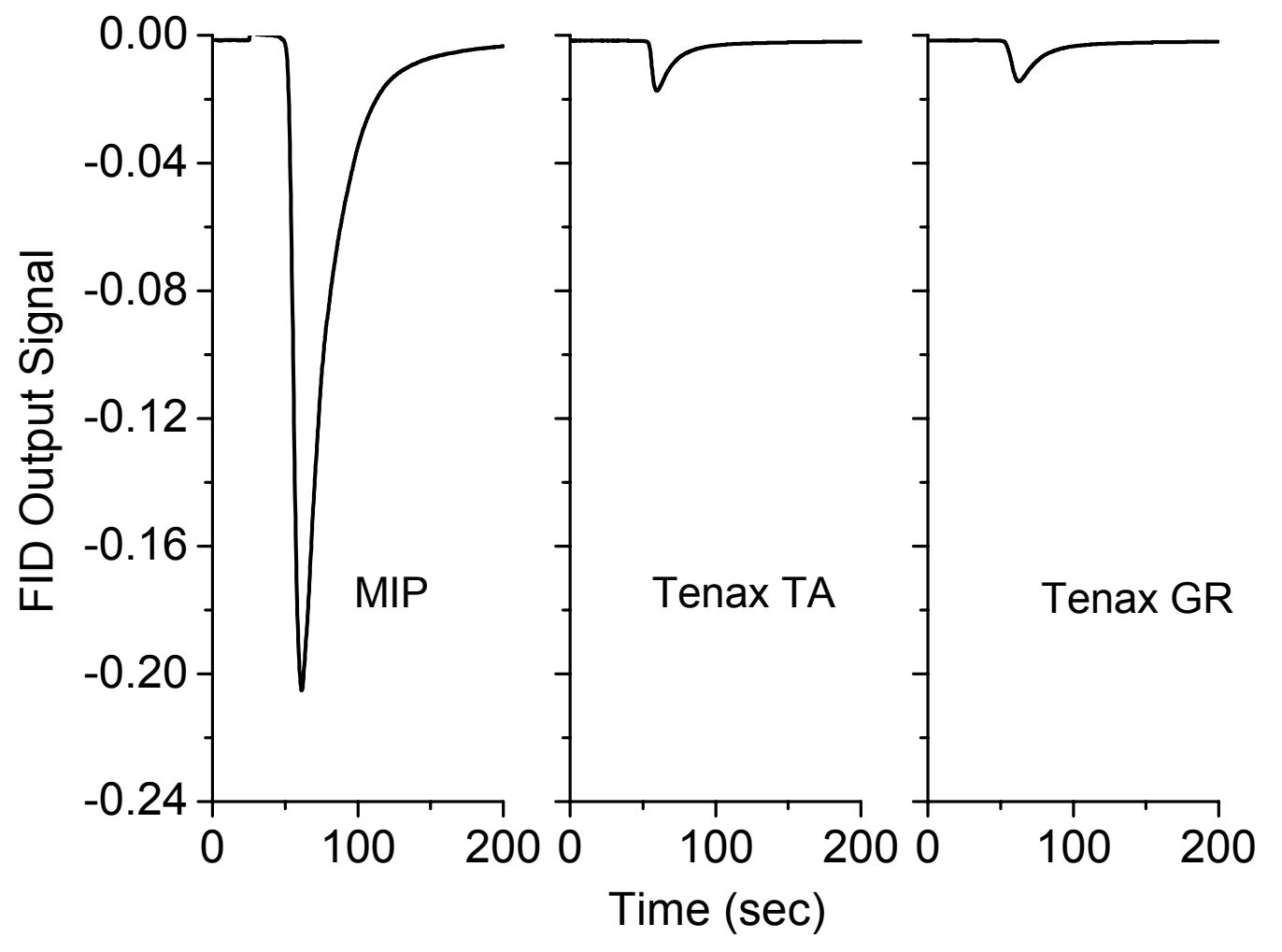

Figure 2.26. Thermal desorption profiles toward TOL vapor with different adsorbents. Vapor concentration: 10 ppm; Preconcentrator type III; Flow rate $10 \mathrm{ml} / \mathrm{min}$; Packing amount : $10 \mathrm{mg}$. 


\subsection{Reproducibility and Aging Effects}

Reproducibility in terms of peak height is excellent. Response profiles are highly reproducible among replicate exposures, with relative standard deviation of the maxima of four replicates at a given concentration typically ranging from $0.6 \%$ to 3.5 $\%$. One set of data from 10 thermal desorption pulses with constant adsorption time is summarized in Table 2.8. Also, as shown in Figure 2.27, the desorption profiles produced for successive adsorption/desorption cycles are quite reproducible.

The short term stability of the preconcentration system is tested by repeated measurement of a standard atmosphere under identical conditions over a few days. In this instance, the peak height (desorption maximum) is used. Although a systematic study of stability is not conducted, the results of repeated tests performed 1 week apart for several designs with packed adsorbents show that peak maxima are generally within $15 \%$ of initial values (Table 2.9). Commercial adsorbents show a higher stability compared to the synthesized PPY polymer and MIP materials. The data in Table 2.9 provide some indication of adsorbent stability over extended periods of frequency use (10-20 desorption cycles/day). In addition, comparisons of peak maxima measured before and after repacking preconcentrator type III show that values are within $15 \%$, while the type II design shows a fairly large variation (12 -25 \%). The results demonstrate the ability to periodically replace a PCT(type III) adsorption tube without significantly changing instrument performance. 
Table 2.8 Reproducibility data of PPY film test

\begin{tabular}{c|cccccc}
\hline PF & 37.0 & 36.8 & 36.2 & 36.7 & 37.4 & 37.3 \\
\hline FWHM (sec) & 0.62 & 0.60 & 0.64 & 0.64 & 0.64 & 0.64 \\
& & & & & & \\
\hline PF & 37.5 & 36.8 & 36.2 & 36.8 & 36.6 & 37.0 \\
\hline FWHM (sec) & 0.66 & 0.60 & 0.64 & 0.64 & 0.64 & 0.60 \\
\hline Avg. of PF & \multicolumn{7}{c}{36.9} \\
\hline RSD (\%) & \multicolumn{7}{|c}{1.2} \\
\hline
\end{tabular}

Table 2.9 Stability of Preconcentrator Design ${ }^{\dagger}$

\section{a. Preconcentrator type II}

\begin{tabular}{c|c|c|c|c}
\hline Adsorbent & PPY film & PPY powder & Tenax TA & Tenax GR \\
\hline $\begin{array}{c}\text { Percent change (\%) } \\
\text { of peak maxima from } \\
\text { its initial value }\end{array}$ & 23 & 12 & 9 & 11 \\
\hline
\end{tabular}

b. Preconcentrator type III

\begin{tabular}{c|c|c|c}
\hline Adsorbent & BIP(HQ) & Tenax TA & Tenax GR \\
\hline Percent change (\%) of & & 6 & 8 \\
peak maxima from its \\
initial value
\end{tabular}

\footnotetext{
${ }^{\dagger}$ The measurements were taken on the same system one week apart.
} 


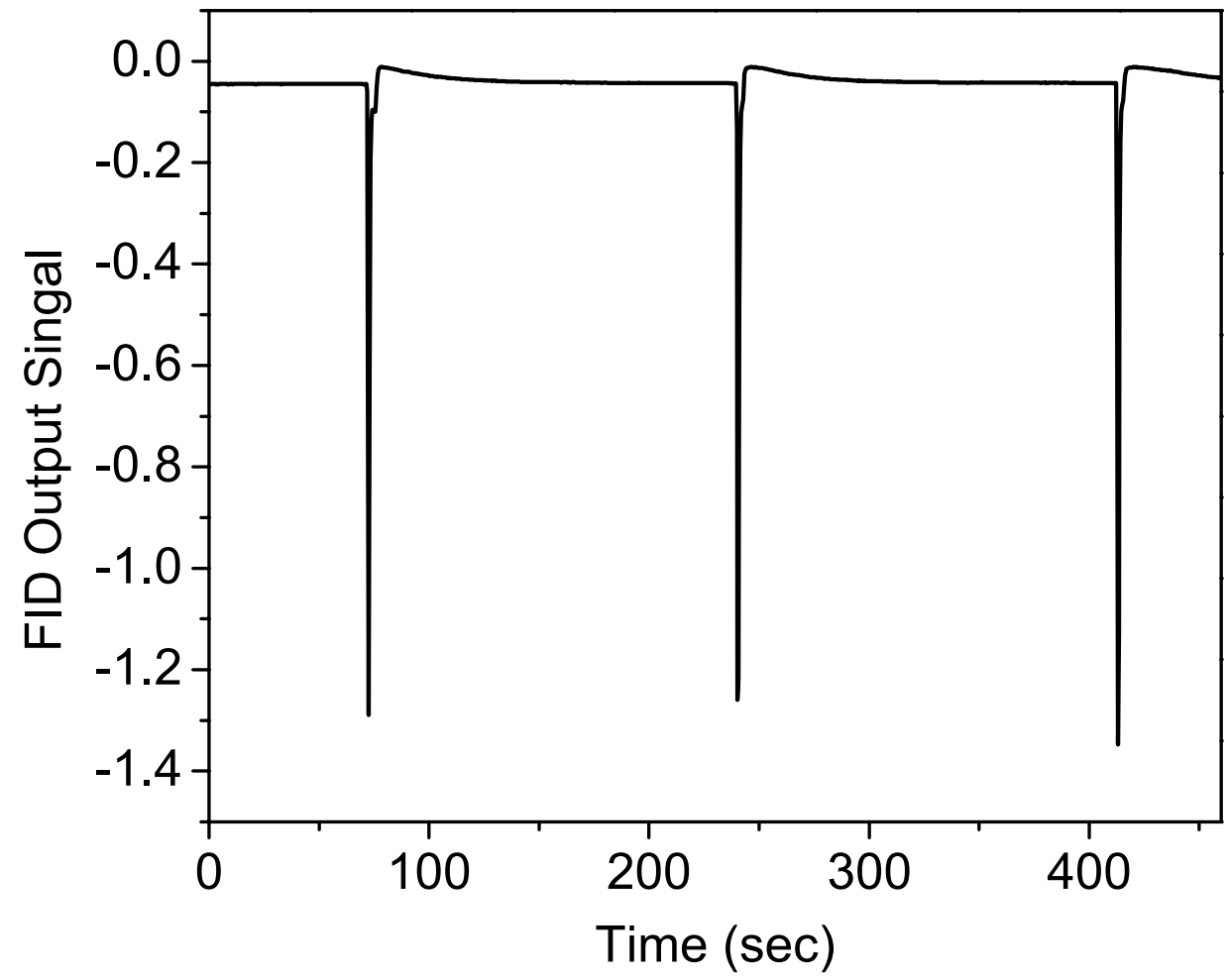

Figure 2.27. A sequential thermal desorption of PCT with PPY powder packing in preconcentrator type II. 


\subsection{Design and Performance Modeling}

Several design issues and factors affecting the PCT performance have been considered and evaluated through the experiment.

The operation of the PCTs is dependent on the thermal desorption heaters. The rate and profile of heating is clearly important. The profile of heating is dependent on the precise characteristics of the heater, the power applied to heater and the control for the heating process. Secondly, the desorption profile is dependent on the characteristics of the vapor and its sorption by the polymer. Third, the profile is dependent on the flow rate between PCT and the detector. Fourth, it may depend on the dead volume of the assembly and the mixing of incoming vapor. All these factors should be taken into account in order to reproducibly fabricate a sampling system with PCTs.

In one design of type II preconcentrators, the Nichrome ribbon is coated with a PPY film to absorb analytes of interest. The suspended membrane structure of the preconcentrator gives it an extremely low heat capacity, which allows for very rapid heating. The application of a current pulse to the heater causes the film layer to heat rapidly and uniformly; this thermally desorbs the collected analytes in a narrow concentrated chemical pulse. However, this type of PCT only shows short term stability. Also the lack of temperature monitoring is a limiting factor.

PCT type III uses an external heater. The Nichrome wire is located outside of the capillary, so heat has to migrate from the external tube wall into the sorbent. The design of PCT is made of capillary tubing so that the unit has low heat capacity and 
can be heated/cooled very rapidly. Use of small quantities of adsorbent would also shortens the heating cycle and decreases the instrument power requirement.

When a given PCT is operating correctly, it produces very repeatable results for several weeks. In many cases, tubes remain in operation until replaced during a repair or maintenance check. When a PCT does not operate properly, it is usually apparent soon after installation. In a few cases, a PCT that have worked fine for weeks inexplicably stopped generating reproducible data. These observations indicate that some work in the design, fabrication and quality control of these tubes is warranted. The heaters do not pose any problem.

Other aspects of sampling systems and connections that requires attention are the flow restriction and leaks. Since the FID signal is very sensitive to flow rate, the designed preconcentrator units in this project are controlled in part by the requirements of the FID. In addition, as flow rate increases, back pressure can build up. Back pressure turns out to be significant across the tubes, particularly if small particles are used. In PCT type II, it is a pain to seal a preconcentrator unit. However, this issue has been well resolved in type III design. Also, reproducibility in packing/retaining the adsorbent in the tube via glass fiber filters can be tricky. All of these issues need to be further investigated in the future PCT development. 


\section{Conclusion}

Several preconcentrators capable of concentrating toluene vapor at low and mid-ppm concentrations are fabricated and tested in this experiment. A variety of adsorbents including commercial solid adsorbents (Tenax GR, TA) and synthesized polymer materials (PPY film, PPY powder and molecularly imprinted polymer) are examined. The design of type II preconcentrator is rather simple and the heating element is in direct contact with packing (coating) adsorbents. Type III design is a miniature preconcentrator, which employs a diaphragm pump, valves and a temperature controller for preconcentration and thermal desorption. Maximum preconcentration factors up to 400 are achieved by packing this type of PCT unit with Tenax GR and Tenax TA. The characteristics of molecularly imprinted polymers as adsorbents in preconcentrator have been evaluated and maximum preconcentration factors on the order of 1000 are obtained. The designed system is portable, stable and performs well as a concentrator for gas phase sensors. 


\section{References}

[1] Pellizzari, E. D.; Carpenter, B.H.; Bunch, J.E. Environ. Sci. Technol. 1975 (9) $556-560$.

[2] Burton, B. Volatile Organic Compounds in Indoor Air Pollution and Health, New York, 1997; pp 127-153.

[3] Johnston, J.J.; Goldade, D.A.; Kohler, D.J.; Cummings, J.L. Environ. Sci. Technol. 2000 (34), 1856.

[4] De. Gouw, J.A.; Howard, C.J.; Custer, T.G.; Baker, B.M.; Environ. Sci. Technol. 2000 (34), 2640.

[5] Matisova, E.; Skrabakova, S. J. Chromatogr. A. 1995 (707), 145.

[6] Fang, M.; Vetelino, K.; Rothery, J.; Frye, G.C. Sensors and Actuators B. 1999 (56), 155.

[7] Harper, M.; J. Chromatogr. A. 2000 (885), 129-151.

[8] Simo, R. J. Chromatogr. A. 1998 (807), 151-64.

[9] Stoev, G. Analytical Letters. 1999, 32(14), 2825-2840.

[10] Ezquerro, O.; Pons, B.; Tena, M. J. of Chromatogra, A. 2003 (1008), 123-128.

[11] http://gc.discussing.info/gs/r hs-gc/microextraction.htm

[12] Wang, J.L.; Chen, W.L. J. of Chromatogr., A 2001 (927), 143-154.

[13] http://www.ss-sci.com/

[14] Pankow, J.F.; Luo, W.T.; Isabelle, L.M.; Sender, D.A.; Anal. Chem. 1998 (70), 5213.

[15] Mitra, S.; Xu, Y.H.; Chen, W.; Lai, A. J. Chromatogr. A. 1996 (727), 111-18.

[16] Lu, C.J.; Zellers, E.T. Anal. Chem. 2001 (73), 3449-57. 
[17] Gawlowski, J.; Gierczak, T.; Pietruszynska, Z.; Gawry, M.; Wohljen, H. Analyst, 2000, 125, 2112-17.

[18] Groves, W.A.; Zellers, E.T.; Frye, G.C. Anal. Chim. Acta, 1998 (371), 131-43.

[19] Nakamoto, T.; I, T.; Isaka, Y.; Ishige, T.; Moriizum, T. Sensors and Actuators B, 2000 (69), 58-62.

[20] Qin, T.; Xu, X. B.; Pacakova, V.; Stulik, K. Chromatographia, 1997 (44), 601604.

[21] Gawrys, M.; Fastyn, P.; Gawlowski, J.; Gierczak, T.; Niedzielski, J. Journal of Chromatogr. A, 2001(933), 107-116.

[22] Baykut, C.; Voigt, A. Anal. Chem. 1992 (64), 677-681.

[23] Wartelle, L. H.; Marshall, W. E.; Toles, C. A.; Johns, M. M. J. of Chromatogr., $A, \mathbf{2 0 0 0}(879), 169-175$.

[24] Wu, J.; Pawliszyn, J. J. of Chromatogr. A. 2001 (909), 37-52.

[25] Sak-Bosnar, M.; Budimire, M.V.; Kovax, S. J. of Polymer Science: Part A:

Polymer Science, 1992 (20), 1609.

[26] Waller, J.; Waren, L.F.; Witucki, E.F. J. of Polymer Science: Part A: Polymer Science, 1998 (26), 1285.

[27] Armes, S. P.; Gottesfeld, S.; Beery, J. G.; Garzon, F.; Agnew, S. F. Polymer, $1991(32), 2325-30$.

[28] Mitra, S.; Yun, C.; J. Chromatogr. 1993 (648), 415.

[29] Kim, M.; Mitra, S.; J. Chromatogr. 2003 (996), 1-11. 